\title{
Dissecting the molecular mechanism and spatiotemporal dynamics controlling senescence entry
}

\author{
Dissertation \\ for the award of the degree \\ Doctor rerum naturalium (Dr. rer. nat) \\ of the Georg-August-Universität Göttingen
}

Within the Doctoral Program

International Max Planck Research School for Genome Science of the Georg-August-University School of Science (GAUSS)

Submitted by

\section{Konstantinos Sofiadis}

Goettingen, December 2019 


\section{Thesis Advisory Committee}

Prof. Argyris Papantonis (Supervisor)

Institute of Pathology, University Medical Center Göttingen

Prof. Ralph Kelhenbach

Institute of Molecular Biology, University Medical Center Göttingen

Prof. Matthias Dobbelstein

Institute of Molecular Oncology, University Medical Center Göttingen

\section{Members of Examination Board}

First Reviewer: Prof Argyris Papantonis (Supervisor)

Institute of Pathology, University Medical Center Göttingen

\section{Second Reviewer: Prof. Nico Posnien}

Department of Developmental Biology, Johann-Friedrich-Blumenbach-Institute of Zoology and Anthropology, Georg-August-University Göttingen

\section{Extended Examination Board}

\section{Prof. Michael Zeisberg}

Department of Nephrology und Rheumatology Universitätsmedizin Göttingen

\section{Dr. Ufuk Günesdogan}

Department of Developmental Biology, Göttingen Center for Molecular Biosciences, University of Göttingen

\section{PD. Dr. Laura Zelarayán-Behrend}

Institute of Pharmacology and Toxicology, University Medical Center Göttingen

Date of oral examination: February $10^{\text {th }}, 2019$ 
Page |3 


\section{Acknowledgements}

The first words of this manuscripts marks the last moments of a four-year journey that started when I first stepped at the Center of Molecular Medicine of Cologne to start my PhD. A journey that did not seem that long, because it was full of priceless experiences, memories feelings and The first words of this manuscript mark the last moments of a four-year journey that started when I first stepped at the Center of Molecular Medicine of Cologne to start my PhD. A journey that does not seem that long, because it was full of priceless experiences, memories feelings and knowledge. Of course, hard moments, stressful days and sad feelings were not absent, but a moment of happiness would always show up to make up for them. This journey offered me the means to satisfy my scientific interests and opened the door to enter the magical world of science. During this $\mathrm{PhD}$ journey a smart person told me that a $\mathrm{PhD}$ is not only about the scientific part- it also about changing you as a human being and making you a better person. I hope that I took a step forward in that direction.

This previous line belongs to Prof. Argyris Papantonis, who offered me the opportunity that I was looking for many months, while anxiety was gradually accumulating. It is very hard to find the exact words to describe and thank him for these years. It is hard to realize if I respect him more for his bright, (not only) scientific brain or for his amazing personality. A wonderful mentor, a role model, a boss with smart motivational skills other than yelling or misbehaving. A glimpse of enthusiasm and interest for your work from his side and suddenly you find yourself on the bench trying harder and harder. Outside of work, a very amusing company. I have learned so much during these years next to you

Fortunately, more people contributed to make my $\mathrm{PhD}$ an amazing experience. I was very lucky to meet very nice people and great instructors. I am very grateful for having Dr. Anne Zirkel, a postdoc back in the lab in Cologne, as a direct supervisor of mine. She really set me in the scientific rails and tried hard to teach me, apart from the scientific part, discipline and responsibility. She was always supervising me with patience and sincere guidance. Her continuous encouragement helped me successfully overcome the difficulties during the beginning of my $\mathrm{PhD}$.

Two meters away from my office, I could always find Dr. Theodore Georgomanolis, our Lab Manager. At least Lab Manager in the beginning, short after a good friend. I learned so many things from Theo and he was always there to help me when I was struggling. Most importantly a fun person to spend some time during the day in order to relax a bit and discuss any random topic.

What should I say for Natasa Josipovic? Anyway, I have learned to be careful with my words around you (kidding!). We started our PhDs almost at the same time and soon developed a beautiful friendship. Always fair, always tough, but paradoxically always sensitive. We were always there to 
support each other scientifically and mentally. Luckily, a third person shared the load of complaints, fun and party nights. Spyridon Palikyras you are a very good friend and a great host. A friend that appreciates all my silly jokes and vice versa.

Of course, I would need several lines to thank all the rest of the members of the lab both in Goettingen and in Cologne. Great scientists, but most importantly very nice people. They were all working hard to establish a professional environment in which each of us could develop individually or as a team. Constant communication and scientific discussion was part of our daily routine, and this taught me so much and really broadened my scientific interests. Finally, the moments we were fed up from work pressure, a call would lead us all to the round table in the kitchen for some coffee and fun discussions. Each one of you played a part in enjoying my $\mathrm{PhD}$ years

I would like to thank my committee members especially Prof. Ralf Kehlenbach and Prof. Nico Posnien for being my TAC, especially Prof. Posnien who agreed to review my thesis. I am also grateful to Prof. Michael Zeisberg and Dr. Ufuk Guenesdogan for agreeing to be on my examination committee.

Outside the scientific community, there were people that really supported me all these years. My best friends "Billares" (Vassilis Nikolaou, Vassilis Tselios) during my bachelor years and up to date, even from far away they are always there when I need them. I know that the plan to do our PhDs at the same place did not work out well, but we managed to find a way to feel close together. Similarly, I want to thank my best friends from my childhood. They are so many and I will probably need unlimited space to write about them. I miss you all guys. Finally, I would like to thank Christina for all the very nice memories and the fun we have had together all these years. I know it was hard (and oftentimes annoying) for you to understand the reasons that kept me so many weekends in the lab, but still you were patient and willing to deal with it.

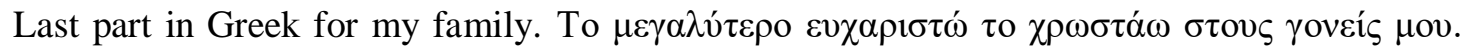

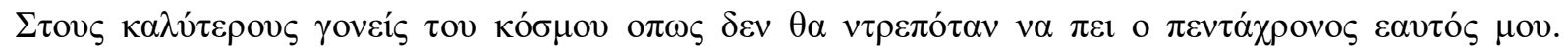

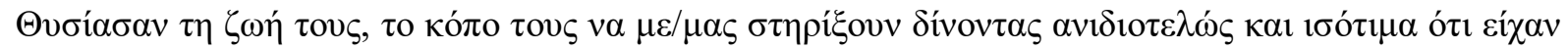

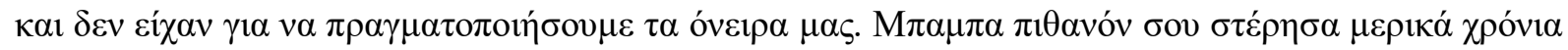

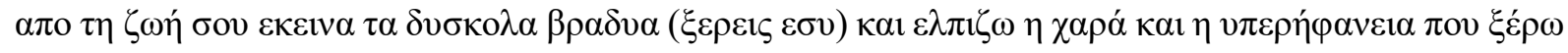

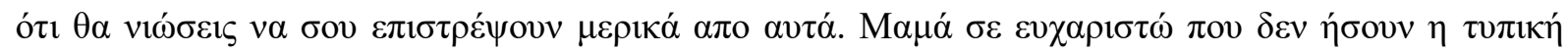

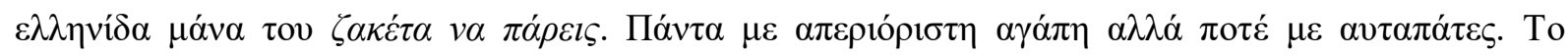

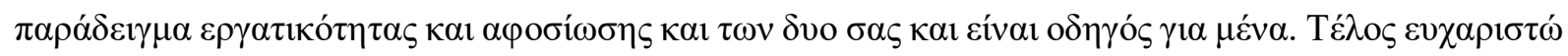

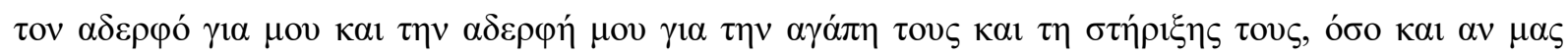

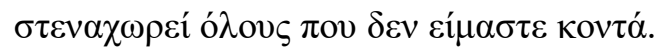




\section{Table of Contents}

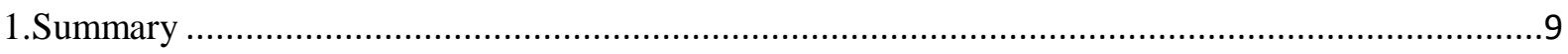

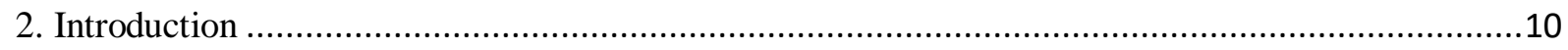

2.1 The three-dimensional genome organization in the eukaryotic cell ........................................10

2.2 The crosstalk between 3D genome organization and transcription ........................................14

2.3 Cellular senescence: A complex cellular mechanism in the defense against tumour as a suitable

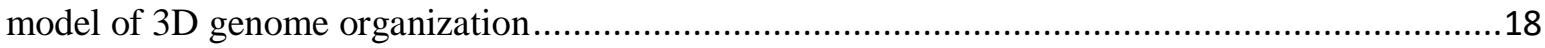

2.3 Cellular senescence is a diverse cellular program with multiple stimuli and signaling pathways

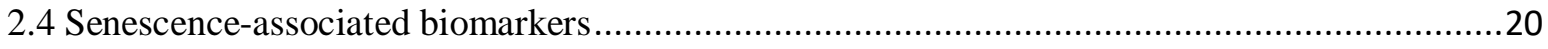

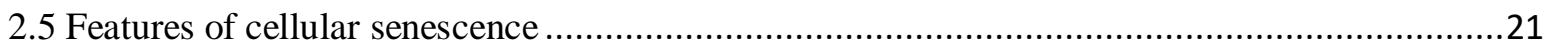

2.6 The interplay between 3D genome architecture and senescence/aging ...................................24

2.7 High mobility group proteins: abundant nuclear protein in the organization pf the genome.... .29

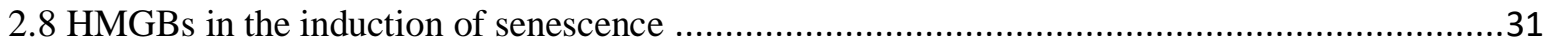

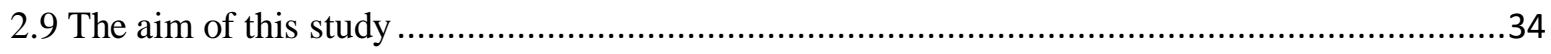

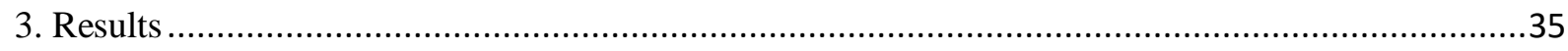

3.1 Chapter I - HMGB2 Loss upon Senescence Entry Disrupts Genomic Organization and Induces

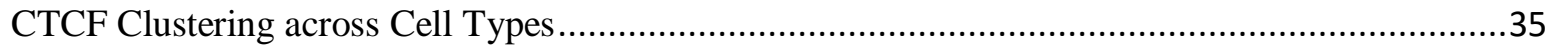

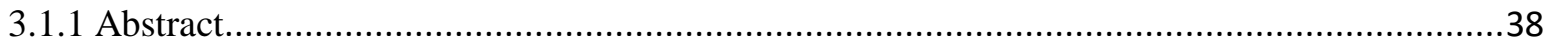

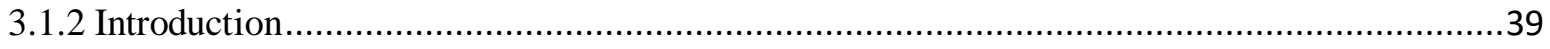

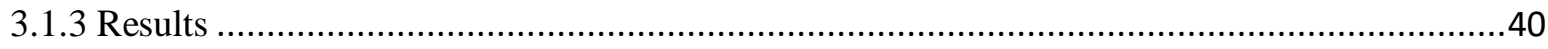

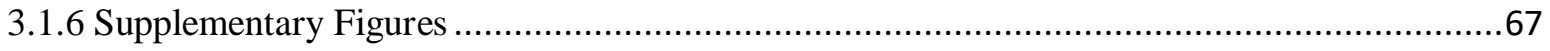

3.2 Chapter II -HMGB1 as a rheostat of chromatin topology and RNA homeostasis on the path to

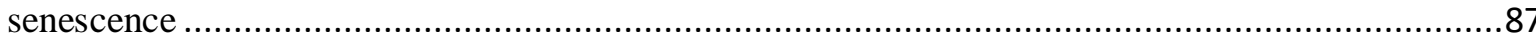

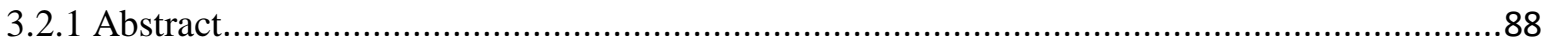

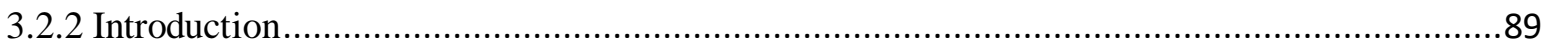

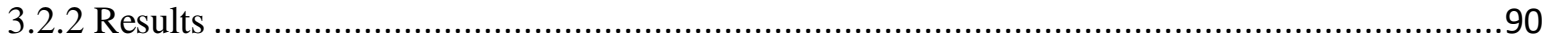

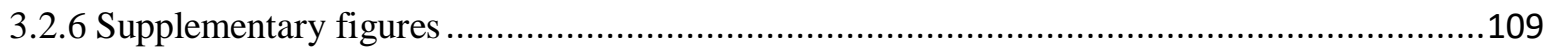

3.2 Chapter III - Repurposing a small-molecule HMGB inhibitor to induce rapid and uniform

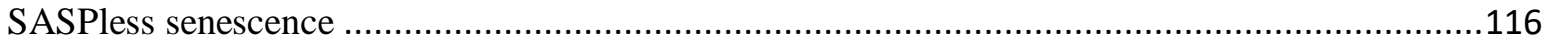

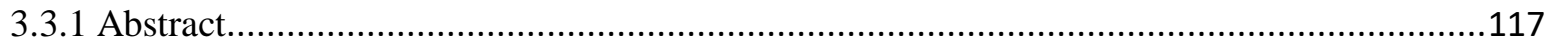

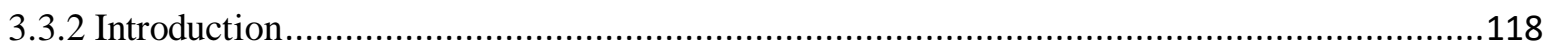

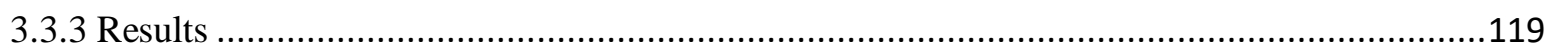

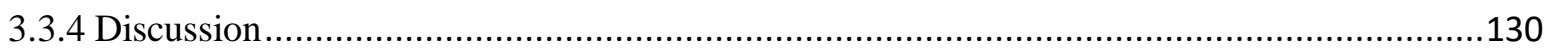

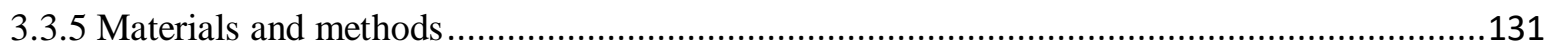

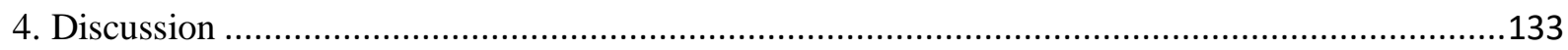


4.1. The loss of HMGBs marks the entry of senescence and coincides with chromatin changes....133

4.2 Genome-wide chromatin re-arrangements dictate the entry of cellular senescence

4.3 The nuclear loss of HMGB2 instructs the senescence associate transcriptional activity and induces rapid genomic re-organization

4.4 HMGB1 complements HMGB1 to TAD demarcation but its nuclear loss is associated with distinct to HMGB1 genomic re-arrangements

4.5 HMGB1 is a bona fide RNA-binding protein regulating the nascent-RNA turnover of SASP factors... .141

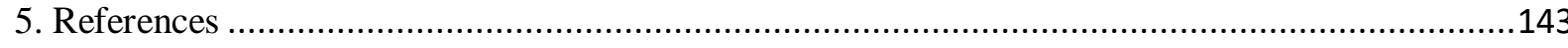

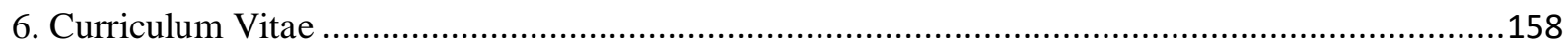




\section{List of abbreviations}

\begin{tabular}{|c|c|}
\hline $3 \mathrm{C}$ & Chromosome Conformation Capture \\
\hline $3 \mathrm{D}$ & 3-dimensional \\
\hline qPCR & quantitative real-time polymerase chain reaction \\
\hline $\mathrm{CDK}$ & Cyclin-dependent kinase \\
\hline CDKN2A & Cyclin-dependent kinase Inhibitor 2A \\
\hline ChIP-seq & Chromatin Immunoprecipitation-sequencing \\
\hline Clip & Cross-linking immunoprecipitation \\
\hline $\mathrm{CpG}$ & C-phosphate-G \\
\hline CRISPR & Clustered regularly interspaced short palindromic repeats \\
\hline CTCF & CCCTC-binding factor \\
\hline DNA & Deoxyribonucleic acid \\
\hline DNA-SCAR & DNA segments with chromatin alterations reinforcing senescence \\
\hline FKBP-Casp8 & FK506-binding protein-caspase 8 \\
\hline GO & Gene Ontology \\
\hline GWAS & Genome-wide association study \\
\hline $\mathrm{HDF}$ & Human diploid fibroblasts \\
\hline HGPS & Hutchinson-Gilford progeria syndrome \\
\hline HMGBs & High Mobility Group Proteins \\
\hline HOXA & Homeobox (gene) A \\
\hline HUVECs & Human umbilical vein endothelial cells \\
\hline ICM & Inflachromeme \\
\hline ISS & ICM-induced senescence \\
\hline $\mathrm{KD}$ & Knockdown \\
\hline $\mathrm{KO}$ & Knockout \\
\hline LAD & Lamin-associated domains \\
\hline OIS & Oncogene-induced senescence \\
\hline PDGF & Platelet-derived growth factor \\
\hline $\mathrm{RB}$ & Retinoblastoma \\
\hline RBPs & RNA-binding proteins \\
\hline RNA & Ribonucleic acid \\
\hline ROS & Reactive oxygen species \\
\hline RPKM & Reads per kilobase million \\
\hline $\mathrm{RS}$ & Replicative senescence \\
\hline SAHF & Senescence-associated heterochromatic foci \\
\hline SASP & Senescence-associated secretory phenotype \\
\hline $\mathrm{SHH}$ & Sonic hedgehog \\
\hline SICC & Senescence-induced CTCF clustering \\
\hline TAD & Topological-associated domains \\
\hline TAF & Telomere-associated DNA damage foci \\
\hline $\mathrm{TGFb}$ & Transforming growth factor beta \\
\hline TIF & Telomere-dysfunction-induced foci \\
\hline TNF $\alpha$ & Tumour necrosis factor alpha \\
\hline TSS & Transcriptional start site \\
\hline WRN & Werner \\
\hline ZRS & Zone of polarising activity Regulatory Sequenc \\
\hline
\end{tabular}




\section{Summary}

Senescence represents a programmed cell cycle arrest against aberrant proliferation and tumour development due to accumulated genotoxic or metabolic stress. The tumour-suppressive properties of senescence attracted the attention of the scientific community and led to pioneering studies to fully understand the rules governing the establishment of this homeostatic mechanism. Several groups attempted to characterize the features of senescence and elucidate the molecular pathways that lead to the transition from a proliferating state and to a senescent one. This attempt led to the characterization of a number of intriguing phenotypes associated with the entry into senescence. Interestingly, it was shown that extensive chromatin re-arrangements and altered genomic conformation is part of these phenotypes and contributes to the inhibition of proliferating genes and expression of senescent ones. Nowadays, the contribution of three-dimensional (3D) genome structure in the orchestration of transcriptional pathways in a cell-type and context-dependent manner is well established. The spatiotemporal crosstalk between regulatory elements, such as enhancers and promoters, requires strict organization of 3D genome structure to reassure coordinated wiring of these transcriptional elements. Disruption of genomic structure leads to aberrant gene regulation and has been linked to various pathophysiological phenotypes. Although it was shown that senescence is accompanied by robust alterations in 3D genome architecture, the molecular events that lead to these alterations and their impact on the establishment of senescence were still elusive, especially in a physiological context like that of replicative senescence.

In Chapter I of this thesis, I studied the contribution of the architectural protein HMGB2 in the alteration of genomic organization and the coordination of transcriptional pathways upon entry into senescence in three different human cell types. In Chapter II, I focused on the multifaceted role of HMGB1 as an architectural and RNA binding protein, as well as a secreted factor to demonstrate its important contribution in the establishment of senescence and the emergence of associated phenotypes. In Chapter III, I exploited a small anti-inflammatory inhibitor that selectively targets HMGBs and studied the impact of its administration on HMGB kinetics and on the pathways they control. I also studied the influence of this drug has on the proliferation capacity of primary and cancer cell types. Collectively, in my PhD work I applied a number of different molecular techniques to fully understand the impact of HMGBs on architectural changes upon entry in senescence, and on the establishment of the transcriptional repertoire linked to either the proliferating or the senescent state. 


\section{Introduction}

\subsection{The three-dimensional genome organization in the eukaryotic cell}

The conventional one-dimensional (1D) representation of the transcriptional process, which assumes the RNA polymerase sliding alongside a respective gene to produce a nascent RNA, which in turn will give rise to a protein product, has changed dramatically over the last decades (Rowley and Corces, 2018). This simplistic view of events leading to protein synthesis could not explain the high complexity of the eukaryotic genomes, with the large number of differentially-expressed genes coordinating multiple cellular functions across tissues. Eukaryotic transcription represents one of the most complex cellular processes, requiring the interplay of various molecules (DNA, RNA, protein factors) in a controlled manner (Rada-Iglesias et al., 2018). Although it is common to associate transcription only with the processivity of RNA polymerases towards production of nascent RNA transcripts, in essence transcription involves all necessary steps that orchestrate co-association of various regulatory elements and of important protein factors to load the polymerase at the transcription start site (TSS). Thus, in order to describe the complex process of transcription, additional dimensions, time and space, need to be considered, and thus increase the complexity of eukaryotic functions from 1D to 3D(Rada-Iglesias et al., 2018).

Human DNA is a long molecule of $\sim 2 \mathrm{~m}$ that needs to be packed in the $\sim 10 \mu \mathrm{m}$ nucleus of a eukaryotic cell. This very remarkable task is achieved through a series of different packaging events (Figure 2.1) that involves the coordinated interplay between DNA and various protein factors (Lawrence et al., 2016). The most abundant eukaryotic proteins -collectively termed histones- possess prominent roles in this process of DNA packaging. Two dimers of $\mathrm{H} 2 \mathrm{~A}$ and $\mathrm{H} 2 \mathrm{~B}$ histones in its interior and two dimers of $\mathrm{H} 3$ and $\mathrm{H} 4$ histones in its exterior form the core of the nucleosome, around which the DNA is wrapped every 146 bp (Lawrence et al., 2016). The fifth member of the family, linker histone H1, binds the nucleosome at the entry and exit sites of the DNA (Hergeth and Schneider, 2015). The extraordinary feature of the histone octamer is that the tails of histone $\mathrm{H} 3$ and $\mathrm{H} 4$ extent outside the nucleosome and are subjected to chemical modifications (Law et al., 2015). A large amount of studies have described the importance of these modifications in a variety of cellular processes, with particular focus in the regulation of transcription (either activation or repression) (Lawrence et al., 2016; Li et al., 2007). Notably, histone tails and their respective modifiers can directly interact with RNA polymerases, various transcription factors and transcription auxiliary proteins affecting the transcription fate of a given genomic locus ( $\mathrm{Li}$ et al., 2007). Thus, DNA packaging mediated by nucleosomes resolves a spatial necessity, whereas histone tails provide an additional regulatory layer to orchestrate the multiple functional outcomes. 


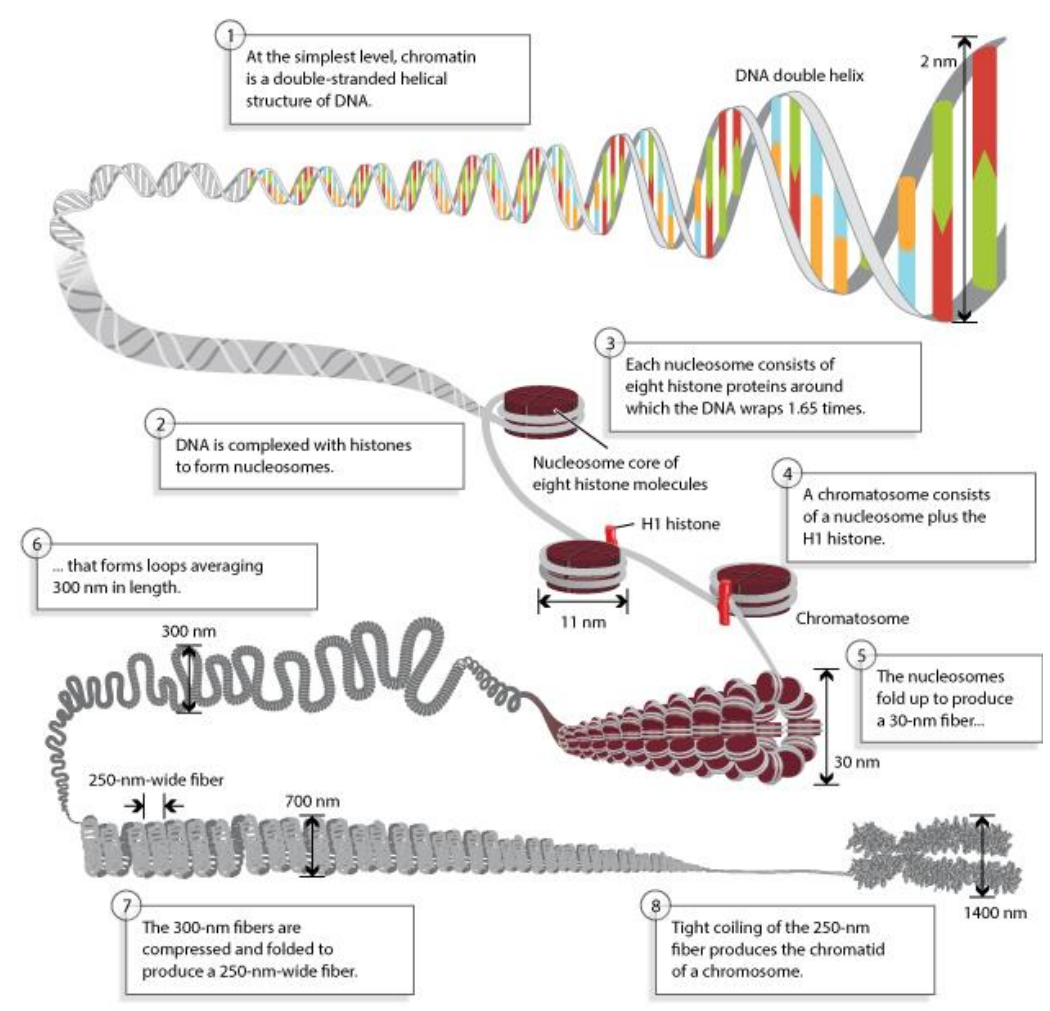

Figure 2.1. The multiple layers of packaging in the eukaryotic nucleus.

Human DNA is a long molecule sized $2 \mathrm{~m}$ that needs to be placed in the nucleus. Mainly histone proteins and other architectural proteins compress DNA in a series of packaging steps to fit DNA in every cell of the human organism. Adapted from (Pierce, Benjamin. Genetics: A Conceptual Approach, 2nd ed).

Although histones are the most abundant proteins residing on DNA, they still only represent a fraction of the numerous protein factors and RNA molecules that either constantly or occasionally bind to DNA. In fact, the macromolecular complex comprised of DNA, RNA and protein molecules collectively termed chromatin. Based on the dense staining under the microscope, Emil Heitz described a very compacted configuration of the chromatin later termed as constitutive heterochromatin. Constitutive heterochromatin, which frequently resides in the transcriptional-depleted nuclear periphery, is characterized by high occupancy of repressive histone marks, absence (albeit not complete) of transcriptional activity, gene deserts and high levels of compaction (Wang et al., 2016). Transcriptionally active chromatin, termed euchromatin, possesses active histone marks, high transcriptional activity, gene-rich regions and lower degree of compaction, thus enabling higher mobility (Rowley and Corces, 2018). Facultative heterochromatin represents an intermediate between the two aforementioned chromatin states, consisting of both active and repressive histone marks and being often poised for transcriptional activation (Saksouk et al., 2015). Chromatin represents one of the most complex macromolecular entities in the nucleus, harboring all the regulatory elements in the linear genomic sequence that serve as a direct or indirect scaffold for gene regulatory machines. Strikingly, these regulatory elements often reside thousands of kilobases apart rendering impossible the explanation of the complicated transcriptional events via conventional 1D models (Rada-Iglesias et al., 2018). The 
latter challenge and the accumulation of the Genome-Wide Association Studies (GWAS) studies pinpointing mutations of intergenic regions as drivers of a plethora of diseases (Maurano et al., 2012; Schaub et al., 2012) have heightened scientific interest towards the organization of the chromatin and the spatial interplay between distant genomic regions.

Colossal progress has been made over the last two decades in understanding the significance of chromatin architecture in the execution of cellular processes such as DNA replication, transcription, DNA repair, etc. Transcription is a suitable and extensively used model to study genomic organization, as it requires the spatiotemporal coordination of cis regulatory elements (enhancers, promoters, TSSs) even located Megabases apart, with trans-acting factors launching transcription of the respective genes. The introduction of chromosome conformation capture (3C) technique by Job Dekker in 2002 (Dekker et al., 2002) was a landmark for a series of subsequent pioneering studies to cross over from the 1D interpretation of the events governing transcriptional regulation to the 3D (Figure 2.2). The highlight of these improvements was the release of the first Hi-C dataset in 2009 (Lieberman-aiden et al., 2009). The continuous improvements of $\mathrm{Hi}-\mathrm{C}$ and its derivatives, in combination with the continuous upgrade of super-resolution microscopy and the development of single-cell techniques, continuously provide new insight into the organization of the chromatin and the control of transcription at a high-throughput scale (Denker and De Laat, 2016; de Wit and de Laat, 2012)

The typical Hi-C workflow involves cross-linking of chromatin, enzymatic digestion with a restriction enzyme and incorporation of biotinylated nucleotides in the newly digested ends followed by ligation. At this step, the ligated products are the result of ligation of fragments that are in close proximity in the 3D space, although they may lie kilobase apart in the linear genomic sequence. The biotinylated ligation products are pulled down using streptavidin beads and are subsequently processed for sequencing. Typically, each ligation product between two fragments represents an interaction and the genome-wide interaction profiles deduced by $\mathrm{Hi}-\mathrm{C}$ are used to define the $3 \mathrm{D}$ folding of the genome. The final output of a Hi-C experiment is a heat map with a plaid pattern containing all the interactions across and within individual chromosomes.

Based on the resolution of the generated map it is possible to visualize certain genomic features. The plaid pattern of a Hi-C map at the Mbp-level segregates the genome into two distinct compartments, termed A and B compartments, with mostly active and repressive properties respectively. These are preferentially found in close proximity and interact with higher frequency in cis (Lieberman-aiden et al., 2009). The segregation of genomic sites in A and B compartments is organized in a lineage-specific manner and is instructed by the respective transcriptional landscape. Higher resolution Hi-C maps allow for visualization of genomic features at sub-Mbp level. Notably the 40-kbp resolution maps generated by Dixon et al (Dixon et al., 2012) led to the pioneering characterization of "topologically associated domains" (TADs) and of TAD boundaries. TADs are genomic domains of high interaction frequency, depicted as well-defined triangles along the diagonal of a typical Hi-C heatmap. Disruption of the 
interaction signal at the corners of adjacent TADs indicates a TAD boundary with insulatory role, i.e. of impeding interactions across TADs. TAD boundaries are thought to represent insulating blocks that restrict interactions and transcriptional crosstalk of regulatory elements with genes in neighboring TADs. TAD boundaries are also characterized by extensive CTCF binding, a well-studied architectural factor that facilitates genome folding through the formation of CTCF-specific loops and enhances insulation at TAD boundaries. CTCF acts synergistically with another family of architectural proteins called cohesins. These are able to slide along and extrude DNA, thus facilitating formation of loops. Remarkably, the aforementioned structural elements are highly conserved across multiple cell lineages or even species (Dixon et al., 2012; Phillips-Cremins et al., 2013; Szabo et al., 2019), indicating that they evolved early in the evolution of eukaryotic organisms (Harmston et al., 2017; Krefting et al., 2018).

A variety of studies in several cell types, species and physiological contexts accentuated the high conservation of the basic architectural features shaping genomic organization in interphase nuclei. On the other hand, during mitosis, the structural organization of the genome collapses and chromosomes acquire a homogenous, locus-independent conformation (Gibcus et al., 2018; Naumova et al., 2013). Remarkably, it was shown that during early prophase TADs lose insulation strength and progressively collapse, the compartmentalization into A and B compartments is lost and site-specific contact frequency decreases especially at longer distances, indicating a swift to a higher compaction (Gibcus et al., 2018; Naumova et al., 2013). This phenomenon gradually increases as mitosis progresses into metaphase, where chromatin reaches its highest compaction. At this point, chromosomes completely abolish their interphase characteristics and acquire a spiral conformation of high compaction to go through mitosis (Gibcus et al., 2018; Naumova et al., 2013). The termination of mitosis and re-entry into interphase is accompanied by rapid re-establishment of genomic organization into TADs and A/B compartments, preserving cellular identity of the parental cells and, thus, cellular functions (Gibcus et al., 2018; Naumova et al., 2013). It is not yet fully determined which are the driving forces and the parental inheritable features that drive this re-establishment of genomic organization, despite the fact that various mechanisms have been proposed (Campos et al., 2014). The idea of mitotic bookmarking, for example, suggests that several proteins are associated with chromosomes during mitosis, acting as architectural buoys responsible for orchestrating the high-order structure of the interphase chromosomes (Festuccia et al., 2017). Taken together, chromatin organization seems an integral part of genomic organization and homeostatic cellular function. 


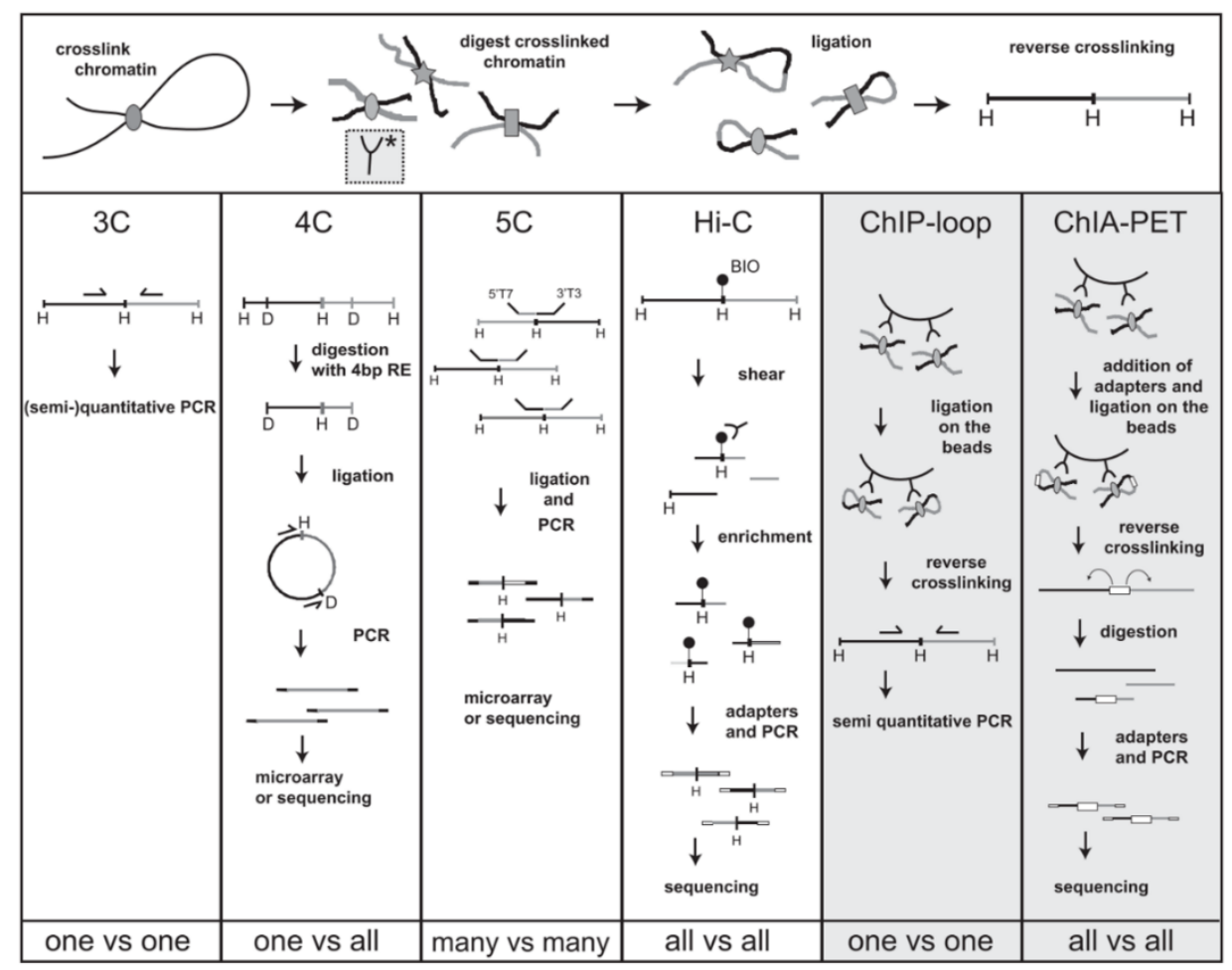

Figure 2.2. The development of chromosome conformation capture studies.

A series of innovative advances led to the development of the genome-wide conformation techniques. The insight acquired from the numerous studies taking advantaging of these methods highlighted the importance of the genomic interactions in the facilitation of the various cellular processes. Adapted from (De Wit, 2012).

\subsection{The crosstalk between 3D genome organization and transcription}

The knowledge obtained from Hi-C studies revealed some of the structural events that dictate the organization of the genome, but the link between structure and function is still missing. Disease- and trait-associated genetic variants identified in large-scale GWAS studies are located with a very high incidence ( 93\%) in non-coding regions (Maurano et al., 2012; Schaub et al., 2012). Understanding the interplay between chromatin organization and its impact on transcription will possibly enable us to characterize the drivers of various pathophysiological states. Point mutations, double-strand breaks, duplications, translocations and inversions are typical drivers for various diseases and the mode of action could be through alterations of the chromatin high-order structure (Krijger and De Laat, 2016). For example, altering the extremities of a TAD or eliminating a TAD boundary could potentially disrupt the cell's specific genomic interactions or create aberrant ones that result in misexpression of a gene or cluster of genes and the development of pathophysiological phenotypes.

Despite the plethora of studies investigating multiple layers to disentangle the functionality of these architectural elements and their associated protein factors orchestrating chromatin organization, the field is far from reaching a unified scenario. Whether TADs and TAD boundaries are important for transcriptional activity, whether loops are a result of de novo formation or pre-exist, and whether loss of 
these elements could promote malignancy are among the questions frequently raised (Schoenfelder and Fraser, 2019). The reality is that during the past decades and with all the innovative technological and experimental advances, remarkable studies form various groups presented convergent and (more frequently) divergent findings.

The initiation of transcription requires dynamic looping of distal regulatory elements, such as enhancers, to their cognate promoters. Frequently, these elements are many kilobases apart and separated by various regulatory elements and genes. Thus, only the proposed model of a partitioned genome with chromatin looping could explain such coordinated crosstalk. The organization of the genomic into TADs, TAD boundaries and CTCF loops, and their influence on transcriptional regulation fit this model. The advent of CRISPR/Cas9 methodology offered a chance to genetically modify these elements in various ways and study the impact on the transcriptional rate. Genomic loci hosting developmental genes require strict control to coordinate their spatiotemporal induction, and this is mediated through a precise crosstalk between transcription factors and the regulatory elements. Due to this fact, these loci were extensively used in the studies of genome architecture. The SHH locus is organized in a tissue invariant and conserved Mbp-longTAD (Rosenbloom et al., 2013) comprised of multiple enhancers responsible for the expression of $S H H$ in a developmental stage- and tissue-specific manner. Interestingly, during mouse limb development, Shh is regulated by the ZRS enhancer, which is found almost $960 \mathrm{kbp}$ away from the gene, bypassing several others in closer proximity (Lettice et al., 2003), (Lettice et al., 2014). Point mutations in the ZRS enhancer suffice to disrupt $S H H$ expression and result in limb malformations (Anderson et al., 2012). These distant regulatory elements translocate into close proximity to drive limb bud expression of $S H H$ (Williamson et al., 2016) and reporter genes inserted in the $S H H$ TAD are efficiently expressed in an $S H H$-similar pattern in the limb under the control of ZRS enhancer (Symmons et al., 2014), (Ruf et al., 2011).

As mentioned before, in order to understand the rules governing 3D genome organization and its impact on transcription many studies modified architectural elements in the Shh locus. The findings obtained from the Shh locus, as well as from other model developmental loci, resulted in convergent and divergent conclusions highlighting that the characterization of a universal description of $3 \mathrm{D}$ genomic organization is a complex task. The following lines provide a brief summary of studies that modified the most important regulatory elements and the impact (if any) in the expression of the respective genes:

Genomic distance: Large deletions of the genomic interspace between SHH and ZRS -but without disrupting the TAD- did not affectZRS-driven expression of $S H H$ in the developing limb showing that this interaction is not distance-dependent (Williamson et al., 2019), (Symmons et al., 2016). This result contrasts with the deletion of the region between Hoxd13 and its limb specific enhancer that resulted in loss of expression (Fabre et al., 2017). The differences in these results might be explained by sequence composition, plasticity of the locus and abundance of structural and regulatory 
elements. Furthermore, higher resolution Hi-C maps might reveal further segregation of the deleted region in sub-TADs or other cryptic regulatory elements.

CTCF sites and orientation: Disruption of a number of CTCF sites surrounding the $S H H$ locus altered the boundaries of the TAD, albeit not generating a completely new TAD. Despite altered TAD margins, the expression of $S H H$ was not affected and all embryos were born without obvious phenotypes (Williamson et al., 2019). Depletion of CTCF sites in the HOXA locus altered its TAD boundary, activating an otherwise silenced downstream gene (Narendra et al., 2015). Additionally, the orientation of the flanking CTCF motifs represents an important property of CTCF binding and loop formation (Rao et al., 2014; Vietri Rudan et al., 2015), as it was shown that a convergent orientation of CTCF motifs holds true for $>65 \%$ of loops formed between two CTCF-bound sites (de Wit et al., 2015). Disruption of this orientation is sufficient to disrupt loop formation or to mediate the formation of differential loops, thus reshuffling local architecture and altering transcription levels of the surrounding genes (Guo et al., 2015; de Wit et al., 2015).

Disruption of a TAD boundary: Genetic modifications of TAD boundaries mimicking human diseases that lead to either complete loss of the boundary and rewiring of TADs led to misexpression of the surrounding genes and recapitulated patient phenotypes in mice (Fabre et al., 2017), (Lupiáñez et al., 2015), (Laugsch et al., 2019). Altering the size of the boundary without affecting the integrity of the TADs or the crosstalk between the nearby genes with the respective enhancers seems to not affect transcriptional output (Despang et al., 2019; Ghavi-Helm et al., 2019; Lupiáñez et al., 2015; Williamson et al., 2019). The technical variations in these experimental procedures explain some of the discrepancies observed, however it seems that complete loss of the boundary and formation of new TADs is critical to observe alterations in the transcriptional status of adjacent genes by introducing new interactions between enhancers and gene promoters.

Loss of architectural proteins: Despite the wealth of studies regarding chromatin architecture and the respective architectural proteins, the influence of these proteins in transcriptional regulation is still not fully delineated. CTCF and cohesin are of the most extensively studied molecules and were suggested to be the main mediators of 3D genome structure and formation of chromatin loops. Full knockout of either the CTCF (Moore et al., 2012) or any of the cohesin (Singh and Gerton, 2015) molecules leads to embryonic lethality, highlighting how essential these molecules are for basic cellular functions. Recently, a “degron" system was introduced to study such essential proteins, taking advantage of a conditional and reversible degradation of targeted proteins using a ubiquitin-ligase system responding to the plant hormone, auxin (Nishimura et al., 2009). Two independent studies induced a complete auxin-mediated degradation of $\mathrm{CTCF}$, resulting in loss of genomic insulation in more than $80 \%$ of TAD boundaries and elimination of CTCF-mediated chromatin loops, illustrating the importance of CTCF binding at TAD boundaries (Nora et al., 2017), (Kubo et al., 2017). Similar auxin-mediated experiments were designed to target cohesin and condensin complexes. Auxin-mediated degradation of 
the cohesin RAD21 (Rao et al., 2017) or SCC1 (Wutz et al., 2017) and a liver-specific, tamoxifeninduced knockout of the cohesin loading factor Nipbl (Schwarzer et al., 2017), resulted in elimination of all CTCF loops, despite CTCF occupancy at its binding sites remaining unchanged. Interestingly, both CTCF and cohesin removal did not completely abolished high-order genomic organization. Notably, A and B compartmentalization remains largely unaffected, with only minor reduction in the strength of compartmentalization upon CTCF degradation (Nora et al., 2017). Compartmentalization of active and repressed chromatin was even enhanced upon cohesin removal, with better-defined and wellseparated A and B compartments. These results suggest that the segregation of chromatin into A and B compartments is an independent process that involves different factors, compared to the genome organization into TADs and CTCF loops.

The aforementioned studies against these architectural proteins raised once more the same question. Since the loss of architectural proteins leads to striking collapse of architectural features, are there any functional consequences in the cell, particularly in the regulation of transcription? Although it has been long suggested that the looping mediated by CTCF/cohesin is the main mechanism for bringing enhancers and promoters in close proximity to initiate transcription, the removal of these important architectural proteins had only a minor effect on gene expression levels. Specifically only prolonged auxin-mediated degradation of CTCF resulted in bulk changes in gene expression, whereas one day of auxin treatment affected only a small number of genes (Nora et al., 2017). Similarly, RAD21 depletion led to a minor upregulation of a subset of genes positioned next to super enhancers while the majority of the genes remained largely unaffected (Rao et al., 2017). Thus, it seems that the absence of these proteins and the subsequent loss of TADs and loops is not sufficient to significantly alter the transcriptional landscape of RAD21-depleted cells. It might be that the structural organization of chromatin had already influenced the establishment of the transcriptional landscape before degradation took place and facilitated the increase in local concentration of transcription factors maintaining the established transcriptional profile even in the absence of these structural factors. Another explanation could be that the limited timeframe of the degradation is simply is not long enough to induce extensive transcriptional changes.

Despite accumulation of data, it is still difficult to establish a universal mechanism that dictates chromatin organization and its downstream coordination of the various transcriptional programs. Possibly, the rules governing the dynamics of chromatin during cellular processes are highly contextand pathway-dependent, rendering the scenario of a universal mechanism unlikely. Since the genomic organization field is relatively young and the methodology still developinig, the produced data need careful interpretation. 


\subsection{Cellular senescence: A complex cellular mechanism in the defense against tumour as a suitable model of 3D genome organization}

Frequently, senescence is conceptually compared to aging, but in reality, senescence and aging represent two related but distinct biological processes that develop both separately or simultaneously and occur at different scales. Accumulating evidence renders cellular senescence as one of the major drivers of accelerated aging and age-related disease. In parallel, there are many reports showing that cellular senescence is more pronounced in the later stages of life as a result of telomere exhaustion, accumulation of DNA lesions, imbalanced homeostasis through a number of diverse stressors that could force cells to enter senescence. Interestingly, the emergence of both senescence and aging, are accompanied by extensive alterations in the 3D genome structure and the levels of various architectural proteins. Thus, both these two biological processes represent a suitable model to study genome architecture in order to delineate some of its underlying rules and the impact of various architectural changes on its initiation, progression and establishment in cells.

\subsection{Cellular senescence is a diverse cellular program with multiple stimuli and signaling pathways}

The first description of cellular senescence emerged in the 60's, with the seminal work of Hayflick and (L. HAYFLICK and Wistnr, 1961), when they noticed that cultured primary cells only have a finite number of division in vitro, although they are still viable and metabolically active. This was the first report of one particular type of cellular senescence, termed replicative senescence. Numerous studies up to date reported a wide spectrum of metabolic and genotoxic stressors, known as potential senescence-inducers (Figure 2.3). The best studied examples include telomere attrition (Cesare and Karlseder, 2012; D'Adda Di Fagagna et al., 2003), DNA damage (D'Adda Di Fagagna, 2008), accumulation of reactive oxygen species (Davalli et al., 2016) and aberrant activation of oncogenic pathways (Lee and Schmitt, 2019). Despite differences in senescence type and phenotype diversity, the various stressors induce the most prevalent feature - irreversible cell cycle arrest. This confers resistance to oncogenic transformation, thus rendering cellular senescence a potent tumour-suppressor mechanism by permanently withdrawing pre-malignant/damaged cells from the cell cycle (Campisi, 2013). Later reports referred to cellular senescence beyond the tumour suppression context in wound healing (Demaria et al., 2014), (Meyer et al., 2016), (Chiche et al., 2017) and embryonic development (MuñozEspín et al., 2013), (Storer et al., 2013). The diversity of inducing stimuli and resulting phenotypes makes identification of a universal pathway in the induction of senescent a very difficult task. However, some basic pathways described involve the action of the tumour suppressors [46]. Although these are 
not the sole acting pathways, the $\mathrm{p} 16^{\mathrm{INK} 4 \mathrm{a}} / \mathrm{pRB}$ and the $\mathrm{p} 53 / \mathrm{p} 21$ pathways (acting synergistically in many cases [47]) are responsible for the induction and the maintenance of many different senescence types.

p16 $6^{\mathrm{INK} 4 \mathrm{a}}$ is a cyclin-dependent kinase that acts as an inhibitor of cyclin D-dependent kinases CDK4 and CDK6, controlling the G1-S phase transition (Beach et al., 1993; Khleif et al., 1996). p $16^{\mathrm{INK4a}}$ is encoded by the $C D K N 2 A$ locus, which is also in close proximity to $C D K N 2 B$, responsible for the expression of INK4a family member, p15 $5^{\mathrm{INK} 4 \mathrm{~b}}$. Furthermore, alternative splicing of the $C D K N 2 A$ results in the production of $14^{\text {arf }}$ suggesting that this chromosomal locus evolved as defense against tumourigenesis. The inhibitory action of $\mathrm{p} 16^{\mathrm{INK} 4 \mathrm{a}}$ towards cell cycle-associated proteins prompt the activation of RB tumour suppressors, which in turn selectively inhibit the S-phase-promoting E2F family genes leading to a stable exit from the cell cycle (Harbour and Dean, 2000). Absence of p16 (Jarrard et al., 1999) or pRB (Sage et al., 2003) is sufficient to bypass senescence and mutations in these genes are frequently encountered in malignant human tumours. On the contrary, ectopic expression of p16 ${ }^{\mathrm{INK4a}}$ (McConnell et al., 1998) or Rb (Bennett et al., 1998) efficiently remove the cells from the cell cycle and induce a senescence-associated program. The role of p21 in senescence and tumour suppression remains unclear and controversial. In consistence with p16, p21 is also a cyclin dependent kinase capable of inhibiting CDK complexes and regulating the progression of the cell cycle (Afshari et al., 1996; Dimri et al., 1996). The ability to control cell cycle and the fact that during persistent DNA damage $\mathrm{p} 21$ is often activated by $\mathrm{p} 53$, led to $\mathrm{p} 21$ as a potential mediator of senescence. Similarly to p16, enforced expression of p21 induces cell cycle arrest (Khleif et al., 1996; Sang et al., 2008). Interestingly, the involvement of p21 in the context of senescence was described in two fascinating studies that drifted away from the ordinary focus of the field. Namely, p21, and not the p53 or p16 branches, seems to be the effector pathway in the induction of senescence in the developing embryonic tissues, and absence of p21 leads to developmental abnormalities (Muñoz-Espín et al., 2013; Storer et al., 2013). The activation of p21 in embryonic tissues is mediated through the SMAD/TGF $\beta$ signaling. In fact, activation of the $\mathrm{SMAD/TGF} \beta$ pathway was observed in senescent cells and was previously associated with increased expression of p21 and cell cycle arrest (Acosta et al., 2013; Datto et al., 1995). Interestingly the $\mathrm{SMAD} / \mathrm{TGF} \beta$ pathway represents a second leg in the senescence associated secretory phenotype (SASP) during Ras-induced senescence driven by NOTCH1 activation (Acosta et al., 2013; Hoare et al., 2016), that effectively expands the senescence phenotype by enhancing expression of p21 in neighboring cells. Collectively this interplay with $\mathrm{p} 21$ and SMAD/TGF $\beta$ suggests a positive feedback loop to reinforce p21-mediated senescence at least in a subset of senescence types. 


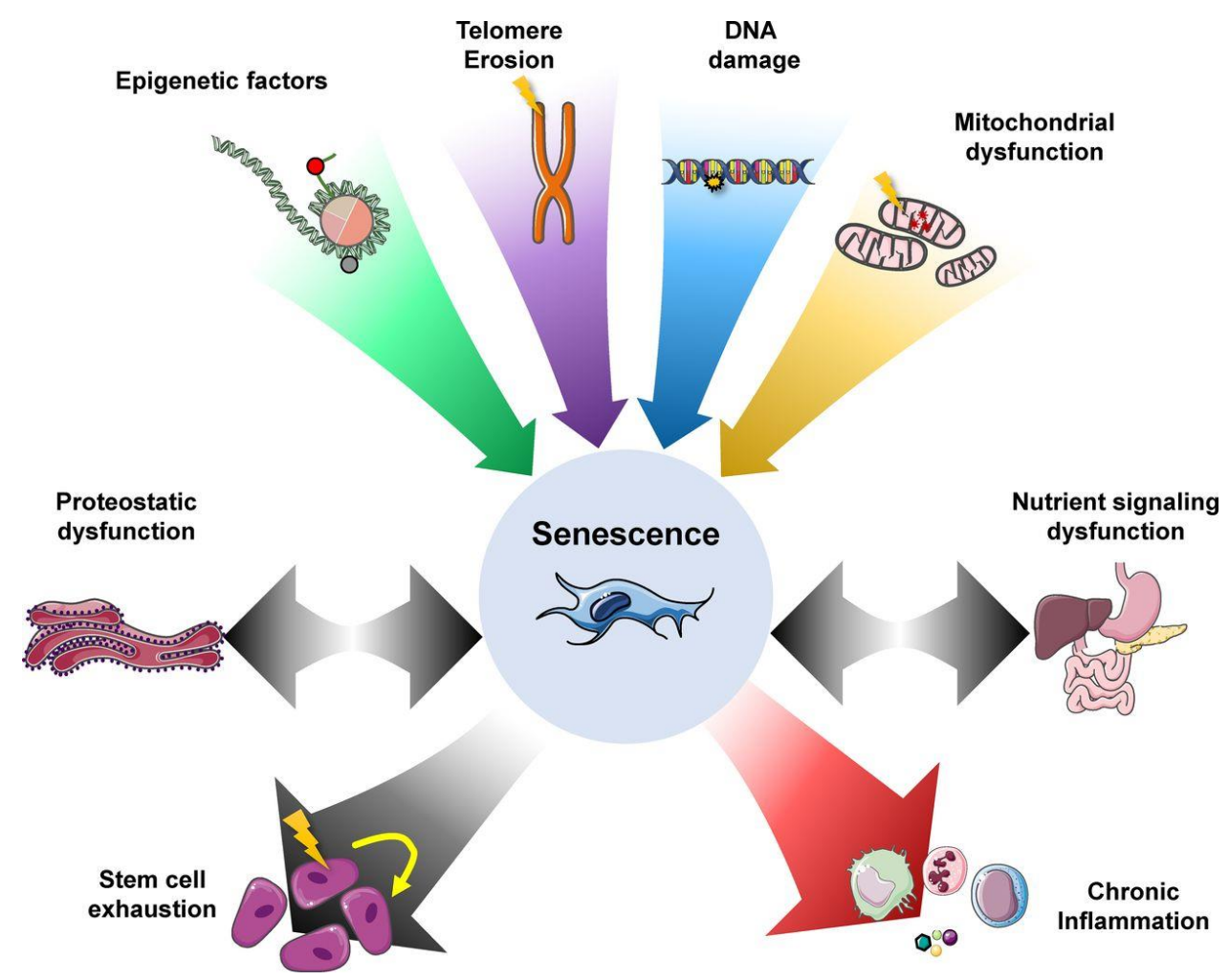

Figure 2.3. The diversity of cellular senescence

Multiple stimuli have been described in the induction of senescence. These different stimuli leads to homeostatic imbalance that eventually is encountered with cell cycle arrest. Persistent and accumulated senescence in turn could detrimentally affect the normal cellular function and promote aging-related disorders and cancer development. Adapted from (McHugh, 2017).

\subsection{Senescence-associated biomarkers}

One of the important obstacles in the definition of senescence is the absence of reliable and universal senescence markers. The absence of such markers makes identification of senescent cells a challenging task, especially in vivo. A wealth of senescence studies over the last years, have provided adequate insight to designate a panel of phenotypical and molecular characteristics (Salama et al., 2014).

Since senescence is by definition a programmed block of proliferation, growth arrest is the most common feature in the diverse senescent contexts and represents a pivotal marker to define senescent cells (Salama et al., 2014) with various proliferation assays commercially available. Likewise, unresponsiveness to mitogen stimulation for re-entering the cell cycle is generally an acceptable marker of senescent cells. Visualization of blue staining through $\beta$-galactosidase assay derived from the senescence-mediated activation of acidic lysosomal b-galactosidase represents the "gold standard" for the identification of senescent cells (Dimri et al., 1995). Similarly, the recently described Sentragor (Evangelou et al., 2017) reacts with and stains blue the cytoplasmic lipofuscin granules that accumulate gradually during progression of senescence. However, efficiency varies due to various factors like the accessibility of the respective cell/tissue, the complexity of the assay and the cross-reactivity with other 
than senescence cellular processes. Morphological changes are also indicative of senescence, as senescent cells generally enlarge, flatten and acquire a non-defined shape with abundant stress granules compared to the proliferating ones (Campisi, 2013). Fluorescence microscopy could be also used to identify markers of senescence through visualization of distinct features organized in the nuclei of senescent cells. The senescence associate heterochromatic foci (SAHF, reviewed in 2.6 in more detail) represents an example of such markers as it describes the formation of discrete foci, enriched with local heterochromatin-associated protein factors that reinforce the repression of proliferating genes in the nuclei of the oncogene-induced senescent cells. Moreover, telomere-dysfunction induced foci (TIF) and telomere-associated DNA damage foci (TAF) form in the nuclei of senescent cells due to telomere shortening and accumulation of DNA damage. Finally, the co-localization of p53 or pRB with characteristic DNA damage biomarkers was observed in the nuclei of senescent cells and termed "DNA segments with chromatin alterations reinforcing senescence" (DNA-SCARS). However, these phenotypical characteristics are far from universal as they were described in certain stresses while they are completely absent from other ones.

A different approach to detect senescent cells is through assessment of expression levels of specific molecular markers such as protein factors involved in the signaling cascades shared with high frequency in the majority of the different senescent types. As it was mentioned before, the most frequently deployed pathways are the $\mathrm{p} 16^{\mathrm{INK} 4 \mathrm{a}}$ and the $\mathrm{p} 21$. The main concerns using these protein factors are that 1) they are not universally expressed in all senescent types; 2) they are expressed both in in vivo and in vitro in non-senescent cells (ironically even in cancer cells); 3) the available antibodies are not very efficient especially when destined for vivo applications. An intriguing epigenetic biomarker of senescence is based on the DNA methylation status of six specific $\mathrm{CpG}$ islands. Surprisingly, the genes hosting these six $\mathrm{CpG}$ islands had little functional relevance due to the low expression levels in most samples tested. Still the levels of methylation, either hyper- or hypo-methylated was predictive for both the state (proliferating or senescence) and the approximate passage number in a cell-independent fashion (Koch et al., 2012).

\subsection{Features of cellular senescence}

One of the major properties of senescence acting as an anti-tumour mechanism is the ability of senescent cells to interact with their microenvironment. Senescent cells actively communicate with their neighboring cells by secreting a variety of molecules, a phenotype collectively termed as senescenceassociated secretory phenotype (SASP) (Coppé et al., 2008) and represents one of the most fascinating features of senescence (Figure 2.4). The SASP repertoire includes a large number of pro-inflammatory cytokines, chemokines, growth factors and proteases. The secretory pathway engaged in the induction of SASP is highly stimuli- and cell type-dependent (Lecot et al., 2016). Despite the long-standing studies 
from various groups, it is still debatable whether SASP actually confers beneficial effects in the coordinated defense against the aberrant proliferation, or the initial burst of persistent accumulation of the secreted molecules could eventually lead to more aggressive tumour (Coppé et al., 2010).

The paracrine properties of the SASP seem important for both the establishment of senescence and its role as a tumour suppressor mechanism. The ability of senescent cells to communicate with neighboring cells represents a potential stress signaling mechanism, which ensures that neighboring cells will be "primed" for defense against a particular stress (Coppé et al., 2010). At least a subset of these SASP factors was shown to reinforce the spread and the maintenance of oncogene-induced senescence through a signaling cascade that eventually activates senescence-associated pathways (Acosta et al., 2008; Coppé et al., 2008; Kuilman et al., 2008). Beyond tumour suppression, SASP possesses further beneficial effects such as the preparation of the damage tissue for repair and acceleration of wound healing (Coppé et al., 2010). It was shown that senescent fibroblasts and endothelial cells transiently accumulate in the sites of wounding where they express an early SASP factor, PDGF-AA (Demaria et al., 2014). PDGF-AA mediates myofibroblast differentiation and enables optimal wound closure. It was shown that myofibroblast differentiation progresses through the expression and accumulation of a matricellular protein CCN1 (Kim et al., 2013). CCN1 was also linked to a p53- and p16-dependent accumulation of senescent cells in the optimal wound closure and the alleviation of fibrosis in the cutaneous wound healing (Jun and Lau, 2010). In fact, the alleviation of fibrosis depends on an additional fascinating feature of senescence. Since senescent cells are capable of secreting a large repertoire of inflammatory agents, it offers the ability of a delicate interplay with the immune system. This is of a great importance as it leads to immune-surveillance and subsequent clearance of senescent and tumour cells (Xue et al., 2007). In fact immune-clearance was also important to diminish both liver and muscle fibrosis (Demaria et al., 2014; Jun and Lau, 2010).

Despite the beneficial aspects of SASP, the secretion of this very diverse repertoire of factors is not devoid of deleterious consequences. Persistent accumulation of senescent cells and chronic inflammation driven by extensive SASP activation could potentially lead to aging-related diseases and to a more aggressive tumour-development (Coppé et al., 2010). The immune-clearance of senescent cells is not without cost, as recruitment of inflammatory cells to the sites of accumulated senescence leads to degradation also of the surrounding healthy tissue (Childs et al., 2015). The loss of healthy tissue and the resulting exhaustion of stem-cell renewal due to the increasing tissue-regeneration need leads to aging-associated phenotypes. Paradoxically, while senescence was described as a tumoursuppressive mechanism, increasing evidence suggests that SASP represents a prominent driver of tumour development and the link between inflammation and cancer has been long established. Multiple SASP factors have been described to increase their concentration leading to destabilization of cellular homeostasis and activation of cancer-related signaling cascades (Childs et al., 2015). Furthermore, 
declining healthy tissue integrity facilitates tumour progression by increasing the invasion and metastasis (Childs et al., 2015).

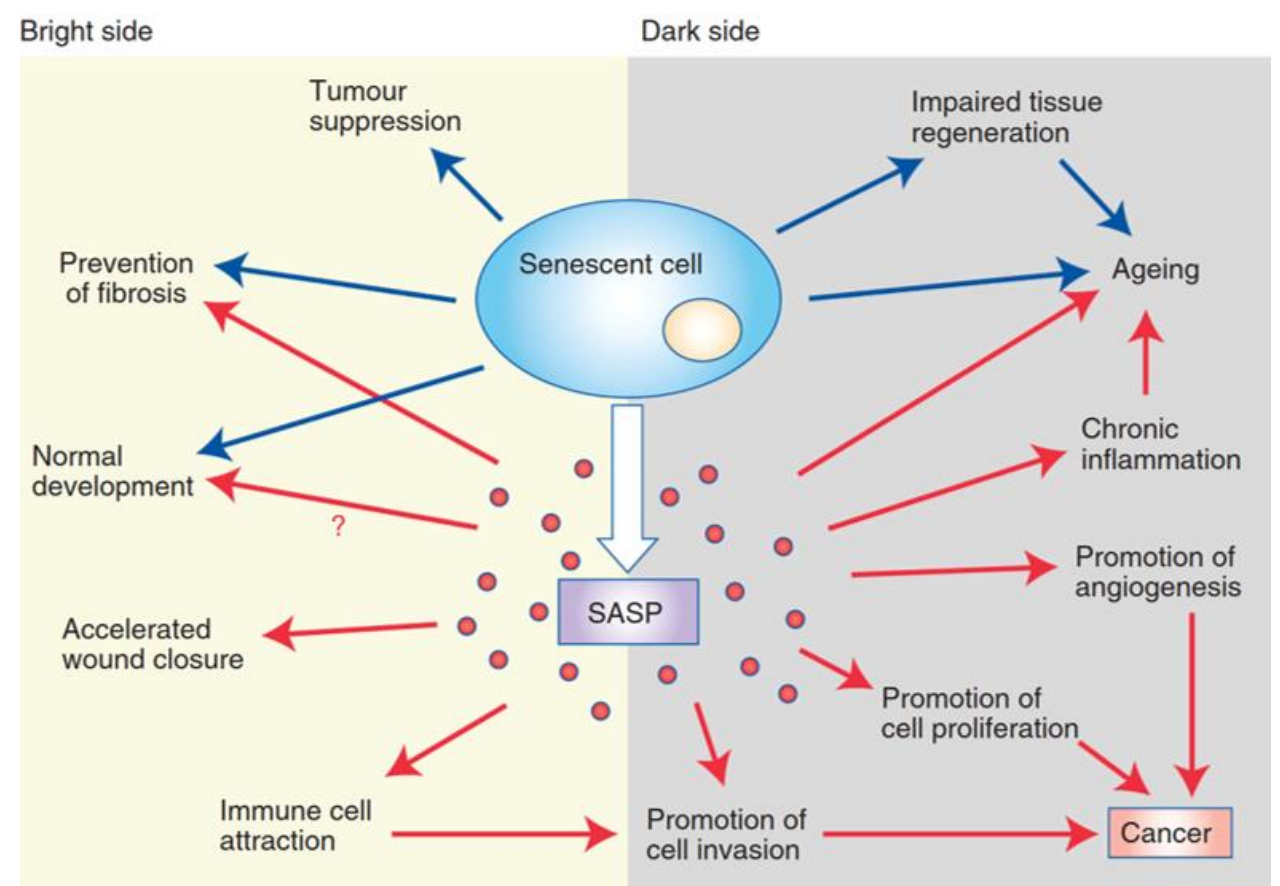

Figure 2.4. The bright and the dark side of cellular senescence.

Cellular senescence has been implicated in various cellular functions beyond the tumour suppressive mechanism. The extensive secretion of the SASP factors in turn promotes detrimental consequences to the cellular homeostasis and contributes to the emergence of age-related diseases. Adapted from (Lecot, 2016)

Despite accumulating knowledge, the complex nature of senescence raises various questions: Does senescence represent a useful molecular mechanism in the defense against cancer and disease? Do its beneficial effects outcompete the detrimental ones so as to consider senescence as a true homeostatic mechanism? Interestingly, senescence has been characterized as one of the hallmarks of organismal aging (López-Otín et al., 2013) and increasing evidence links senescence (mainly via the SASP) with cancer development (Childs et al., 2015). This causal link between senescence and aging has been mainly due to studies showing that clearance of senescence cells is sufficient to increase the lifespan and ameliorate disease-associated phenotypes (Baker et al., 2011; Chang et al., 2016; Jeon et al., 2017). Progeroid mice with a hypomorphic BubR1 (a mitotic checkpoint protein background) were shown to increase healthy lifespan through selective elimination of senescent cells (Baker et al., 2011). BubR1 progeroid mice were carrying an INK-ATTAC transgene comprised of a $16^{\text {Ink4 }}$-specific promoter and a membrane-bound myristoylated FK506-binding protein-caspase 8 (FKBP-Casp8) fusion protein. The activation of the $16^{\text {Ink4 }}$ promoter using a synthetic drug, AP201187 (Baker et al., 2011) drove dimerization of FKBP-Casp8, which targeted p16-expressing cells for apoptosis. Taking into account that p16 is one of the most reliable biomarkers of senescent cells, administration of AP201187 and subsequent expression of the caspase selectively eliminated only the p16-expressing senescent, but not 
non-senescent cells (Baker et al., 2011). Clearance of senescent cells attenuated progression of agerelated disorders, tumour formation and increased healthy lifespan. Similar results were obtained in earlier studies using $C d k n 2 a$-depleted genetic background mice (Baker et al., 2008).

These studies suggested that clearance of senescent cells positively influences organismal homeostasis and inspired senolytic drugs-development to specifically target senescent cells and alleviate senescence-mediated progression of age-related diseases. Reports focusing on senolytic drug development arose from cancer studies, taking advantage of pathways shared between cancer and senescence. The initial attempts of pharmacologically eliminating senescent cells were based on the upregulation of pro-apoptotic B cell lymphoma 2 protein family members. This pathway is frequently upregulated in various cancer types and two therapeutic agents have been developed (ABT263, ABT737) (van Delft et al., 2006; Tse et al., 2008). Later reports proposed these two drugs as senolytic drugs to effectively clear senescent cells both in vitro and in vivo. Likewise, drugs developed to target the expression of p53 drove senescent cells into apoptosis alleviating the pathophysiology of osteoarthritis [82] and increasing the healthy lifespan of either naturally aged mice or in an accelarated aging mice model [83].

\subsection{The interplay between 3D genome architecture and senescence/aging}

One of the underrated features of the work over the last decades to understand senescence is the potential contribution of dynamic alterations in 3D genome structure and epigenetic states towards the induction of cellular senescence and vice versa (Figure 2.5). Evidence of the influential contribution of 3D structure in senescence was presented mainly through telomeric attrition and the DNA damage through double stand breaks. These genomic aberrations were sufficient to activate senescence pathways and lead to cell cycle arrest. In parallel, various chromatin alterations were shown to associate with accelerated aging and aging-associated disorders. Since growing evidence suggest senescence as one of the major drivers of age-relates diseases, the dynamics of genome architecture prior, during, and after induction of senescence represent an explored filed that attracts the attention of scientific community. Despite emergence of sporadic reports, the rules governing the organization of genome organization upon senescence and the key factors involved in this still remained elusive.

The first description of the interplay between the 3D structure and senescence came by Narita et al back in 2003 (Narita et al., 2003). Using oncogene induced senescence triggered by Ras activation, they showed a discrete DNA formation in the nuclei of senescent cells, visualized as punctuated DNA foci originating from DAPI-dense staining in fluorescent microscopy (Narita et al., 2003). These DAPIdense foci were termed as "senescence-associated heterochromatin foci" (SAHF) as they were enriched with constitutive heterochromatin marks such as H3K9me3 and HP1a (Narita et al., 2003), and 
facultative heterochromatin markers such as H3K27me3 (Chandra et al., 2012) and macro H2A (Zhang et al., 2005). Interestingly staining of the H3K9me3 and H3K27me, prominent histone marks of constitutive and facultative heterochromatin respectively, develop a very defined SAHF appearance. In immunofluorescence stainings, SAHF foci are organized as concentric rings of H3K9me3 in the core, and $\mathrm{H} 3 \mathrm{~K} 27 \mathrm{me} 3$ in the surrounding of the ring. The formation of these heterochromatic rings during senescence, suggests that the transition from the proliferating into the senescence state is also accompanied by dynamic re-arrangements of high-order structure (Chandra and Narita, 2013). In fact, it was shown that this nuclear accumulation of repressive histones is not a genome-wide spreading of heterochromatin (Mikkelsen et al., 2007), but rather a re-arrangement of the existing histone pattern (Chandra et al., 2012).

The description of SAHF formation during senescence was a fascinating finding but still, the link between SAHF and the events triggering senescence was missing. Whether this very dynamic reorganization of chromatin dictates the events leading to senescence or is a downstream effect is still not clear. Notably, it was shown that the rearrangement of the histone marks during the transition from proliferating to senescence is closely linked to the switch of the transcriptional program governing each state. Genes involved in cell cycle progression and DNA replication, as those of the E2F family that regulate the S-phase of the cell cycle, are embedded into SAHF (Chandra et al., 2012). The increase of heterochromatic histones on proliferating genes coincides with their simultaneous dissociation from key senescent factors in order to activate their transcription, as was shown for the $\mathrm{p} 16^{\mathrm{INK} 4 \mathrm{a}}$ locus (Agger et al., 2009; Barradas et al., 2009). Interestingly, deletion of p16 or its downstream target pRB inhibits the formation of SAHF (Narita, 2007). On the other hand, enforced expression of p16 is sufficient to activate expression of RB, SAHF formation and down-regulation of cell cycle-specific E2F genes (Narita, 2007). Altogether, this suggests a positive feedback loop between chromatin organization and orchestration of transcriptional programs governing senescence.

Interestingly, redistribution or fluctuation of histone marks and respective histone modifiers were documented in both aging and aging syndromes across species. Aged fibroblasts resulted in reduced $\mathrm{H} 3$ and $\mathrm{H} 4$ biogenesis and protein levels due to chronic DNA damage from telomere shortening (O’Sullivan et al., 2010). Similarly to SAHF, redistribution of histone marks especially of H3K9me3 and H3K27me was also reported to premature aging diseases (Sun et al., 2018). In the Werner syndrome, mutations in the $W R N$ gene, an helicase involved in DNA repair, leads to premature aging (Zhang et al., 2015). WRN interacts with H3K9me3-methyltransferases SUV39H1 and HP1a - and depleted interaction in Werner patients leads to decreased levels of $\mathrm{H} 3 \mathrm{~K} 9 \mathrm{me} 3$ and subsequent heterochromatic loss (Saksouk et al., 2015). In the Hutchinson-Gilford progeria syndrome (HGPS), an LMNA-deficient progeroid syndrome (Eriksson et al., 2003; De Sandre-Giovannoli et al., 2003), a heterochromatic loss due to decreased H3K9me3, H3K27me3 and their respective HP1a and EZH2 methyltransferases, was also observed (Dechat et al., 2008; Jackson et al., 2006; Shumaker et al., 2006). Intriguingly, all these 
heterochromatin-associated factors gradually decrease during human lifetime leading to a progressive heterochromatic loss. Generally, heterochromatic loss leads to genomic instability, accumulation of DNA lesions and aberrant proliferation of cancer-related genes. Taken together, redistribution of epigenetic marks seems to play a pivotal role in senescence, aging and aging-related diseases.

Nuclear lamina plays a prominent role in the organization of the genome as they tethers DNA to the nuclear periphery. It was shown that this attachment is not organized randomly, but specific genomic regions of the genome preferentially interact with the nuclear lamina, termed Lamin-associated domains (LADs) (Guelen et al., 2008; Pickersgill et al., 2006). LADs possess increased levels of heterochromatic marks and are responsible to keep genome integrity by keeping the silenced chromatin compacted in the nuclear periphery (van Steensel and Belmont, 2017). The changes in the cell morphology with enlarged nucleus observed in both HGPS and senescence, is a result of disrupted laminar integrity. LMNB1 is used as a molecular biomarker robustly down-regulated and degraded through autophagy as cells enter senescence (Freund et al., 2012; Shimi et al., 2011). Upon senescence entry, these heterochromatic regions become detached from the nuclear laminar due the loss of LMNB1 thus resulting in a general re-arrangement of the chromatin, which partially dictates formation of SAHF (Chandra et al., 2012). It seems that LMNB1 is necessary for the formation of SAHF but not sufficient, as enforced depletion of LMNB1 does recapitulate SAHF (Chandra et al., 2012). The depleted nuclear lamina-integrity explains some of the observed phenotypes similar to senescence in the pathophysiology of HGPS. Absence of LMNA during HGPS leads to abnormal cellular morphology, accumulation of DNA damage and re-distribution of heterochromatin from the periphery to the interior similarly to the OIS. Interesting apart from LMNA, decrease LMNB1 levels are also observed in HGPS. Collectively, genomic alterations observed in senescence and aging are also induced by the weakening of nuclear lamina.

The advent of 3C-based technologies enabled two research groups to investigate the interplay between chromatin structure, cellular senescence and aging. Particularly, they performed Hi-C using distinct types of senescence and attempted to shed light in the dynamic alterations of 3D chromatin structure changes observed by microscopy and chromatin immunoprecipitation. Notably, Chandra and colleagues (Chandra et al., 2012) took advantage of RAF1-oncogene induced senescence that generates a homogeneous senescence population with pronounced SAHF formation (around 86\% SAHF-positive cells). By obtaining such a large number of SAHF-positive cells, they were able to focus on the most prominent senescent-associated feature related to genome architecture. Interestingly, the increased compaction seen during formation of SAHF was not apparent in their Hi-C data, as they observed a significant loss of genomic interactions within individual TADs and loss of local insulation. The sequence composition of the regions losing insulation is associated with high LMNB1 occupancy and low GC content, features that seem quite important in local de-compaction. Notably regions with LMNB1 occupancy and low GC content were highly predictive of the loss of local interactions across 
the genome and the increase of distal ones. The latter finding and the observation that these regions were significantly enriched with $\mathrm{H} 3 \mathrm{~K} 9 \mathrm{me}$, but not $\mathrm{H} 3 \mathrm{~K} 27 \mathrm{me} 3$, is at least in part reminiscent of SAHF formation.

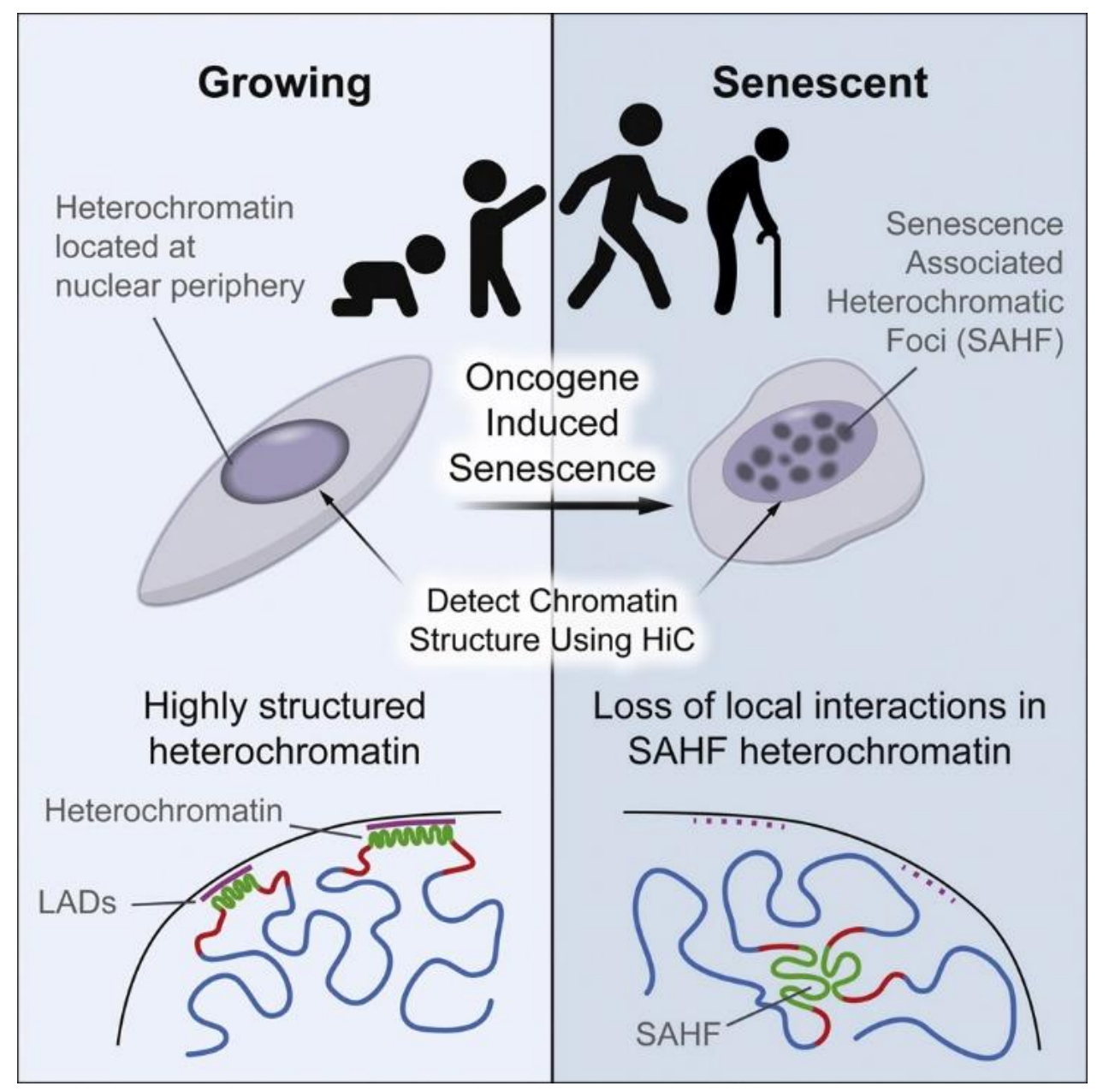

Figure 2.5. Chromatin architecture is strongly linked with the senescence profile.

Re-distribution of the chromatin in the nucleus of senescent cells formation of the SAHF foci. A coordinated crosstalk of the chromatin fiber with the lamina ensures the compaction of the heterochromatin in discrete foci in the nucleus to selectively repress genes associate with the proliferating stage. Adapted from (Chandra, 2015).

A later study by Criscione and colleagues (Criscione et al., 2016), published results convergent and divergent to that by Chandra et al regarding the chromatin organization of the senescent state. It is important to mention that they obtained "deep" senescence by culturing human diploid fibroblast (HFD) cells until replicative exhaustion and they harvested them for Hi-C after an additional four months. Thus, this type of senescence varies considerably from the senescence type that Chandra et al used that relies in short-term activation of RAF. In contrast to Chandra observation, the produced Hi-C of the deepsenescent cells showed a genome-wide decrease of long-range interactions and gain of short-range ones. Nevertheless, the changes in the interaction landscape were also associated with genomic features like enrichment in promoter sequences, high GC content and LAD-regions. One possible explanation for the 
observed discrepancy is that the chosen time-points represent distinct phases in the induction of senescence. The early time-point possibly represents early events after the induction of senescence, whereas culturing the senescent cells for many months highlights the events governing the maintenance and the adaptation of the cells to the senescent state.

Despite differences in the local connectivity observed by the two groups, there were features of the high-order structure shared in the two datasets. Despite changing insulation within and among TADs during transition to the senescence state, the vast majority of TADs remain invariable between growing and senescent cells. This is not surprising as TADs are highly conserved across cell types as different as stem cells and their downstream derived cell lineages (Dixon et al., 2012, 2015). Even though the absence of changes in TAD boundaries could imply a static scheme in the interplay of chromatin and senescence, this is not actually precise. There is significant switching of numerous TADs between the active A compartment and the repressed B compartment and vice versa. In fact, this compartmentalization switch of TADs explains, at least in part, the transcriptional switch correlated to either growing or senescent state. Indeed, around $20 \%$ of these TADs encompass genes associated with cellular proliferation, p16 upregulated pathways and chromatin architecture. Interestingly a high percentage of genes related to growing or senescent state were in stable A compartment, thus their regulation does not seem to be dependent on the compartmentalization

Taken together, despite discrepancies, the generation of Hi-C data from growing and oncogene/deep replicative-induced senescence pinpoint dynamic changes in genomic organization. However, there are some caveats centered in these experimental setup. Namely, in the Chandra study they took advantage of a non-physiological system as they employed oncogene-induced senescence, which induces rapid senescence and thus rapid alterations in the organization of the genomic structure. Similarly, SAHF that represents an explicit manifestation of chromatin alterations has only been identified in oncogene-induced senescence and has not been observed in any other physiological in vitro or in vivo to date. On the other hand, in the Criscione study they used replicative senescence, which represents a physiological system to study senescence. The drawback of this study lies in the fact that they performed Hi-C after four months of the timepoint they defined as the entry into senescence. Therefore, they drew an interaction landscape in cells that already went through all the necessary homeostatic adjustments to adapt in the senescent state. Hence, they could not investigate the dynamic events of an ongoing transition from the proliferating to the senescent state and define how dynamic alterations in 3D genomic structure instructs development of the senescent landscape and vice versa. 


\subsection{High mobility group proteins: abundant nuclear protein in the organization pf the genome}

The high mobility group box (HMGB) proteins constitute a family of very abundant and ubiquitously expressed non-histone proteins that bind to the minor groove of DNA and influence multiple processes such as transcription, replication, recombination, DNA repair and genomic stability (Štros, 2010). They belong to the superfamily of the high mobility group (HMG) proteins, which subdivide into three distinct structural families: the HMGA (HMG-AT-hook-family), HMGN (HMG nucleosome binding family) and HMGB (the HMG-box) (Reeves, 2010) . Members of each family are abundantly and ubiquitously expressed across eukaryotic cells, exhibiting different structures and unique DNA and chromatin binding motifs. HMGBs named after their high electrophoretic mobility in triton-urea gels due to their high content of positively and negatively charged residues (Goodwin et al., 2000). The HMGB proteins are the most abundant of the HMGs superfamily and consists of four members: HMGB1-3 share an 80\% identity containing two HMG boxes and a long acidic tail and differ mainly in the length of the acidic tail (Figure 2.6). HMGB4 ( 21 kDa) is less well-studied and carries two HMG boxes, but lacks an acidic tail. The vast of majority of HMGBs is localized in the nucleus, but the high occurrence of lysine residues in their protein sequence render them susceptible to acetylation and drives their translocation to the cytoplasm. Interestingly, in many instances, cells actively secrete HMGBs in the extracellular space and it was shown at least for HMGB1 that this can be critical for the proinflammatory response (Davalos et al., 2013).

The structure of HMGB proteins is highly conserved in higher eukaryotes. HMGB1-3 are 25 $\mathrm{kDa}$ proteins consisted of two L-shaped HMG boxes (each one around 80 aa) arranged in tandem and of a long acidic tail in its carboxyl-end. HMGB1-3 share an 80\% identity and differ mainly in the length of the acidic tail which differentiates to some extend the unique functions of each protein. Each HMGBbox is comprised of three positively charged $\alpha$-helices, which serve the DNA binding capacity with limited or no sequence specificity. Notably, they bind into the minor groove of non-B-type DNA structures with high affinity and distort DNA by bending, looping or unwinding. The other main feature of HMGBs, the negatively charged acidic tail, resides in the carboxy-terminus and consists of an array of glutamic and aspartic residues (up to 30). The acidic tail interacts with the binding surface of the HMG-boxes and modulates the binding affinity and functional properties of HMGBs. The length and the sequence of the acidic tail was proposed to result in different biological functions for each protein and is thought to enhance transcriptional activity explaining why HMGB4 acts as a repressor in the testis (Catena et al., 2009). Strikingly, and in a sharp contrast with the rest of HMGBs, HMGB1 contains a cytokine activity domain lying in the B-box and a RAGE receptor-binding domain explaining why HMGB1 is the only characterized secreted factor capable of interacting with surface receptors to contribute to inflammation. 
HMGBs bind DNA with minimal sequence preference in a "hit-and-run" fashion making the interaction, albeit efficient to enhance the transcriptional output. The transient nature of this interaction and the incompatibility of these proteins with commonly used fixatives (Teves et al., 2016) renders the capture of their actual binding sites genome-wide challenging. Three mechanisms have been proposed for the control of the transcription by HMGBs (Štros, 2010). The first one suggests that HMGBs binds to relevant to transcription factor sites and distorts chromatin to make it accessible for the respective transcription factors. The second one suggests that minimal transcription factors binding acts as docking points attracting HMGBs, which in turn distort chromatin and promote bulk binding of the respective transcription factors. The final model involves formation of a protein complex between HMGBs and transcription factors and chromatin bending follows (Štros, 2010).

Although in vitro experiments in cultured cells show functional redundancy between HMGB1 and HMGB2 (Bianchi and Agresti, 2005; Štros et al., 2009), in mice loss of HMGB1 cannot be substituted by HMGB2 suggesting functional diversity to multicellular organisms (Calogero et al., 1999; Ronfani et al., 2001). The functional diversity of HMGBs, despite their high sequence and structural similarities, is also made evident by their different expression in adult tissues. Although all four HMGB members are highly abundant during embryogenesis, they differentiate their expression in adult tissues. HMGB1 is still abundantly expressed in the majority of adult tissues, while HMGB2 is restricted to lymphoid organs and testes. HMGB3 is also down-regulated after embryonic development and sustains expression in hematopoietic stem cells, primitive progenitor cells, and differentiating erythroid cells in the adult mouse cells, where it controls differentiation of myeloid and B-cells (Nemeth et al., 2003, 2005). Finally, HMGB4 is also present in some stages of embryonic development and is only present in adult testes, alongside HMGB2, where it acts as a potent transcriptional repressor (Catena et al., 2009).

The importance of these abundant proteins is reflected on the severe phenotypes their absence lead to in knockout mouse models. HMGB1-knockout mice suffer from severe hypoglycemia and die after one day of birth, while intraperitoneal injection of glucose can only sustain them alive for a couple of weeks underlining the essential role of HMGB1. HMGB2-knockout mice are still viable, but male mice have reduced fertility due to germ cell degeneration and immotile spermatozoa, in line with the restricted presence of this protein in the adult testes (Ronfani et al., 2001). Finally, HMGB3-knockout fail to produce efficiently myeloid and B-cell due to overproduction of erythrocytes (Nemeth et al., 2005). These mice do not possess overt phenotypes as the overproduction of erythrocytes compensates the less efficient differentiation of the downstream cell lineages. 


\subsection{HMGBs in the induction of senescence}

As mentioned before, the 3D structure of chromatin is tightly linked with the events leading to senescence regardless of it being a cause or a consequence. There is sparse information for the role of the HMG proteins in the context of senescence. Interestingly, initial reports showed that HMGA proteins are necessary and sufficient for both the induction of RAS oncogenic senescence and SAHF formation (Narita et al., 2006). In congruency with their role as architectural proteins, the nuclear signal of HMGA proteins in immunofluorescence stainings specifically co-localized with HP1a, marking chromatin organization in SAHF. Although HMGAs were linked to transcriptional enhancement, it seems that their selective binding to the SAHF facilitates spreading of heterochromatin and repression of E2F cell cycle genes to maintain proliferative arrest.

There are also studies focusing on the role of HMGBs in the induction of senescence. The subcellular localization and the presence of HMGBs in the extracellular milieu seems to play multiple roles in the induction of senescence both in a cell-autonomous and in a non-cell autonomous fashion. In sharp contrast to HMGAs, HMGBs were shown to switch off their transcriptional activity and selectively translocate out of the nucleus robustly decreasing their levels. A striking feature of this translocation to the extracellular milieu is that HMGB1 can selectively bind to inflammatory receptors, acting as SASP factor, enhancing the expression of other inflammatory factors. Senescent cells induced by four different stimuli (replicative exhaustion, p16 overexpression, X-irradiation, RAS overexpression) led to the p53dependent release of HMGB1 in the extracellular space as part of the SASP program (Davalos et al., 2013). The p53-dependency of HMGB1 release highlights the contribution of HMGB1, since p53 is one of the most prominent protein factor employed in senescence induction (Rufini et al., 2013). The idea that HMGB1 functions as a SASP factor was also reinforced by the ability of HMGB1 to bind RAGE and TLR receptors and launch a proinflammatory transcriptional cascade controlled by these receptors (Ellerman et al., 2007). 


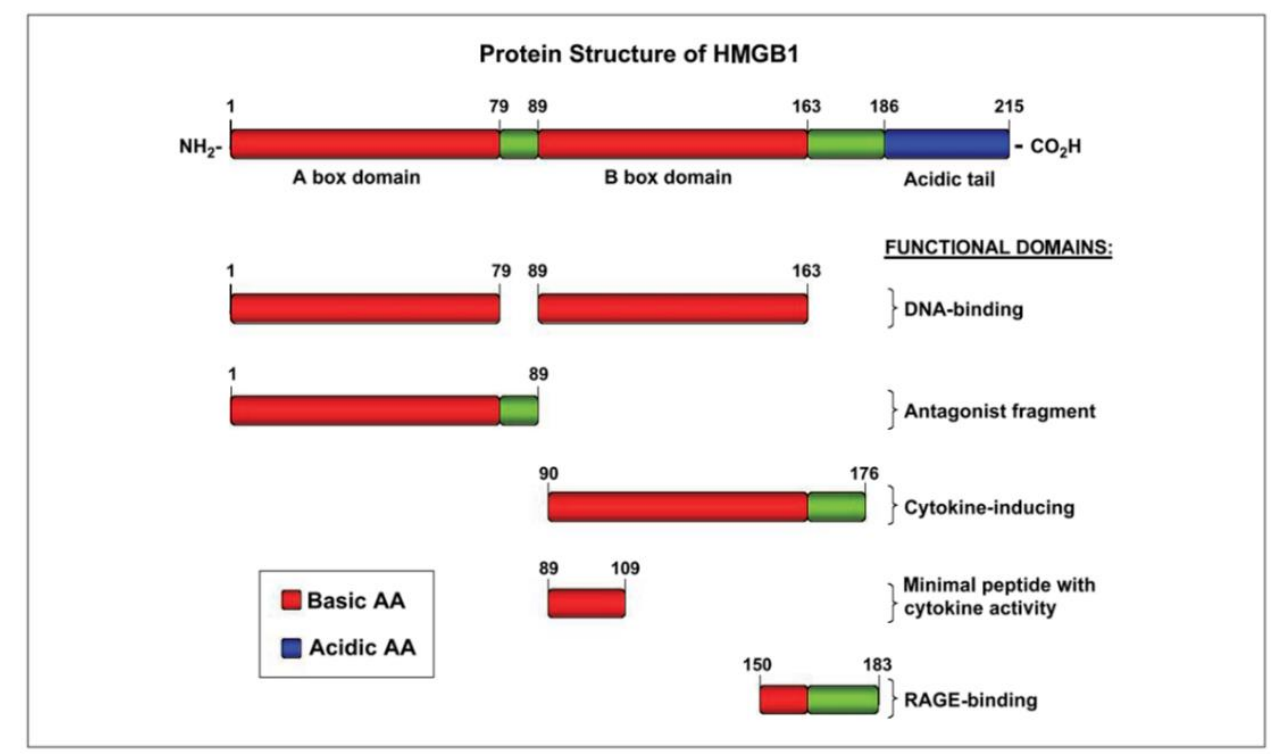

Figure 2.6. Schematic representation of HMGB1 functional domain.

HMGBs are very similar in the protein sequence and their respective functional domains. HMGB1-3 possess the two boxes responsible for the DNA binding ability and the acidic tail in the carboxyl terminus. Cytokine activity and a RAGE-binding domains are uniquely found in the HMGB1 and render this protein as a SASP factor. Adapted from (Watanabe 2017)

Much like HMGB1, HMGB2 also actively dynamically decreases its nuclear levels upon entry into senescence (Aird et al., 2016). The effect of HMGB2 nuclear eviction in individual cells and its potential paracrine effects as a SASP factor remain understudied. Despite the high sequence similarity of the two proteins, HMGB2 does not possess a cytokine-like domain and there is no described role of HMGB2 as a direct ligand in pro-inflammatory receptors up to date. The only contribution to SASP assigned to HMGB2 is through its main role as a chromatin factor and transcriptional regulator. Notably it was proposed that while HMGB2 significantly reduces its levels during senescence, any residual protein selectively bind SASP genes and promote their transcriptional activation (Aird et al., 2016). Although this is an interesting finding, the strength of the ChIP-seq peaks and the low abundance of HMGBs in senescence cells suggest that further investigation into this HMGB2 function is required. Further investigation into the role of HMGB2 is needed to fully understand its role in the induction of senescence, especially as an architectural factor.

The link of HMGBs to senescence could be postulated through the fact that overexpression of these proteins was found in various human tumours like including breast carcinoma, melanoma, gastrointestinal stromal tumours, colon carcinomas and acute myeloblastic leukemia (Štros, 2010). Considering the role of senescence as tumour-suppressive mechanism and the loss of HMGBs upon the entry into senescence, it could be inferred that nuclear HMGB levels could act as a rheostat of cellular proliferation. Whether this could be mediated by their main role as architectural proteins or by any of their other roles is still unclear. Interestingly HMGB1 expression levels are regulated by p53 and the two proteins physically interact in cell nuclei, where HMGB1 promotes phosphorylation and 
subsequentactivation of p53 (Krynetskaia et al., 2008). Finally, an anti-apoptotic role was assigned to HMGB1 through its interaction with, and modulation of the levels of pro-apoptotic factor BCL-2 (Krynetskaia et al., 2008). Bypass of apoptosis is a prerequisite for tumour development, thus, overexpression of HMGB1 could modulate resistance to tumour development. Further investigation needed to elucidate whether the $a b$ nucleo role of HMGBs as architectural factors could orchestrate a tumour-associated transcriptional repertoire and lead to aberrant proliferation

Taken together, HMGBs are versatile proteins capable of translocating from their main subcompartment (nucleus) into the cytoplasmic and extracellular milieu affecting diverse cellular pathways. Although, the vast majority of HMGBs are localized in the nucleus, fluctuations of their levels could initiate different signaling cascades and affect cell homeostasis. Similarly, it seems that there exists a correlation betweem HMGBs nuclear levels and the proliferation capacity of cells. While it was already shown that HMGB levels decrease considerably upon senescence, the actual mechanism and the molecular pathways they impact on, especially as architectural proteins, are still elusive. 


\subsection{The aim of this study}

The role of chromatin architecture in the orchestration of the cellular functions and their potential implication in various pathophysiological situations brought the chromatin architecture studies to the forefront of modern molecular biology. This $\mathrm{PhD}$ thesis attempts to shed some light to the complex topic of chromatin organization and describe some of the rules governing organization of transcription and its subsequent effect on cellular homeostasis. To this end, I exploited cellular senescence, a physiological process in mammals where primary cells progressively switch from a hyperactive and proliferating state to a more metabolically-muted and proliferative arrested one. It is already established that this dynamic and coordinated switch is accompanied by major changes in multiple transcriptional pathways. Despite the fact that various studies showed that chromatin organization changes dictate the transcriptional fate of the growing and senescence states, there were still large gaps on how 3D chromatin structure affects the onset and the maintenance of cellular senescence. This represents the main question of this work focusing on how genome-wide chromatin organization is linked to each of these diverse cellular states and whether changes in this organization suffice for driving the different transcriptional programs.

I specified the main question on the role of the abundant architectural proteins in the nucleus of the proliferating cells, HMGBs. The main reason for this is that, while it was already shown that these abundant proteins are actively evicted from nuclei during the transition into senescence, their role as architectural proteins in this context was completely unexplored. The few previous reports for HMGBs in senescence is focusing more in their role as SASP factors in the extracellular space rather than as transcriptional and architectural regulators in their main compartment of action. Thus, the second question of our study of this work is concluded on how the architectural proteins shape the organization of chromatin in proliferating cells and impacts on the transcription and how their eviction alters the structure and the senescent transcriptional output. Towards this aim, we are going to identify other architectural proteins that function in synergistically with HMGBs

Finally, despite the fact senescence represents model that we integrate our basic research for chromatin dynamics we are willing to contribute to the field of cellular senescence, which is extensively studied yet not completely understood. Senescence is linked to age-related diseases and more permissive tumour development, and despite the remarkable studies, a number of different challenges render senescence as open field for studying. 


\section{Results}

\subsection{Chapter I - HMGB2 Loss upon Senescence Entry Disrupts Genomic Organization and Induces CTCF Clustering across Cell Types}

This project was conceived and coordinated by Dr. A. Zirkel, Dr. M. Nikolic and Dr. A. Papantonis. The manuscript was written by Dr. A. Papantonis. I was involved in the majority of the experimental procedures and I undertook the revisions to successfully publish this manuscript.

My contribution for this manuscript includes the following experimental procedures

- Culturing the IMR90's and HUVECs to replicative senescence and performing the senescenceassociated assays.

- Preparing the samples and performing the Western blots, qRT-PCRs and immunofluorescence stainings

- Knock-down of HMGB2 and downstream applications (RT-PCR, Western blots, Immunofluorescence stainings)

- Optimizing and performing Chip-seq targeting CTCF, HMGB1 and HMGB2

- Co-immunoprecipitation of HMGB2 followed by Western blot

- Hi-chip targeting the interacting sites bound by CTCF

- Rescue experiment with use of piggy-bac expression and the downstream applications (Immunofluorescence, Western Blots)

The following figures and tables were summarized and prepared by A.Z, M.N and A.P using data resulted from my experimental procedures:

- Figure 3.1.1D: Characterization of the protein levels of multiple proteins shows gradual changes in 5 different passages from proliferative to the senescent state

- Figure 3.1.3C: Immunostainings of HMGB1 and HMGB2 in proliferative and senescent IMR90's and HUVECs show the nuclear decrease of these proteins the entry of senescence, a phenotype that is tightly linked with increased nuclear size

- Figure 3.1.3D: Immunostainings of HMGB1 and HMGB2 in proliferative and senescent IMR90's and HUVECs show the nuclear decrease of these proteins the entry of senescence

- Figure 3.1.3E: Immunostainings of HMGB1 and P21 in proliferative and senescent IMR90's and HUVECs show the correlation of HMGB2 decrease and p21 increase upon the entry of senescence

- Figure 3.1.5A-D: Identification of HMGB2 binding sites genome-wide. HMGB2 is mostly bound on gene promoters and gene-bodies and is associated with active transcription. HUVECs 
and IMR90s share a small number of overlapping peaks, a finding that is in line specific epigenetic state of each cell type

- Figure 3.1.5E: Mapping of HMGB2 binding sites over the Hi-C maps generated by A.Z and M.N, show the binding profiles HNGB2 in respect to TADs, TAD boundaries and CTCF.

- Figure 3.1.5G: Correlation of the differentially expressed genes with the alterations observed in TAD level and HMGB2 binding sites upon the entry of senescence

- Figure 3.1.5F-H: Mapping of the HMGB2 binding sites over the Hi-maps and correlation with the TADs margins and CTCF binding shows that HMGB2 demarcates TAD boundaries and the insulation is lost upon the entry of senescence

- Figure 3.1.6D: HMGB2-depleted nuclei drive the formation of CTCF clustering in both HMGB2-knockown and senescent cells

- Figure 3.1.7A-B: HMGB2 and CTCF alignment in respect to the generated Hi-C maps identifies the loops mediated by these proteins and the collapse of these loops upon the entry of senescence

- Figure 3.1.7E: Hi-chip for the interacting regions bound by CTCF reveals the interplay between CTCF and HMGB2 in the formation of loops

- Figure 3.1.7F: CTCF Chip-RT-PCR reveals that the absence of HMGB2 does not affect the binding profile of CTCF in the existing sites and do not form new ones, thus it suggests that the CTCF clustering is a result of CTCF co-localization

- Figure 3.1.7G: Re-introducing HMGB2 with the expression vector decreases the formation of CTCF foci and highlights the tight link between HMGB2 loss and CTCF clustering upon senescence

- Figure 3.1.7I: Expression profiles of architectural and senescence-associated factors in cancer cells upon HMGB2 knockdown

- Figure 3.1.S1B: Differential expression profiles of architectural and senescence-associated factors comparing senescent over proliferative MSCs

- Figure 3.1.S1H: Timecourse expression profiles of architectural and senescence-associated factors comparing senescent over proliferative HUVECs

- Figure 3.1.S2G: Chip-seq targeting H3K27ac and H3K27me3 shows differential binding profiles in proliferative and senescent HUVECs

- Figure 3.1.S7: The impact of HMGB2 in the organization of the proliferative genome and the associated transcriptional pathways

- Figure 3.1.S8: The positioning ofHMGB2 in respect to TAD boundaries

- Figure 3.1.S9A: knockdown of HMGB2 does affect the levels of CTCF and HMGB1 and does not suffice for the emergence of $\mathrm{p} 21$ and positive B-galactosidase staining

- Figure 3.1.S9E: Knockdown of HMGB2 promotes accumulation of the heterochromatic marker HP1a 
- Figure 3.1.S9F: Nuclei depleted by HMGB2 either in senescent cells or in knockdown cells promoted the formation of chromatin-bound CTCF clustering

- Figure 3.1.S9K-L: CTCF Co-Immunoprecipitation shows absence of interaction between HMGB2 and CTCF. RAD21 a known CTCF protein partner used as a control

- Figure 3.1.S9M: HMGB2 Co-Immunoprecipitation identifies the protein partners of HMGB2. GO terms analysis was used to characterize the pathways they are involved

- Figure 3.1.S9N: CTCF Chip-qPCR demonstrates that the CTCF clustering is not a result of de novo CTCF binding to HMGB2 sites or to neo-CTCF sites

- Figure 3.1.S10F: Hi-Chip demonstrates when the insulation of HMGB2 is lost, CTCF is able to perform larger loops explaining in part the CTCF clustering 


\title{
HMGB2 loss upon senescence entry disrupts genomic organization and induces CTCF clustering across cell types
}

\author{
Anne Zirkel, ${ }^{1,10}$ Milos Nikolic, ${ }^{1,10}$ Konstantinos Sofiadis, ${ }^{1,10}$ Jan-Philipp Mallm, ${ }^{2}$ Chris A Brackley, ${ }^{3}$ \\ Henrike Gothe, ${ }^{4}$ Oliver Drechsel ${ }^{4}$ Christian Becker, ${ }^{5}$ Janine Altmüller, ${ }^{1,5}$ Natasa Josipovic, ${ }^{1}$ Theodore \\ Georgomanolis, ${ }^{1}$ Lilija Brant, ${ }^{1}$ Julia Franzen, ${ }^{6}$ Mirjam Koker, ${ }^{7}$ Eduardo G Gusmao, ${ }^{1,8}$ Ivan G Costa, ${ }^{8}$ \\ Roland T Ullrich, ${ }^{1,7}$ Wolfgang Wagner, ${ }^{6}$ Vassilis Roukos, ${ }^{4}$ Peter Nürnberg, ${ }^{1,5,9}$ Davide Marenduzzo, ${ }^{3}$ \\ Karsten Rippe, ${ }^{2}$ and Argyris Papantonis ${ }^{1,11, *}$ \\ ${ }^{1}$ Center for Molecular Medicine Cologne, University of Cologne, 50931 Cologne, Germany \\ ${ }^{2}$ German Cancer Research Center \& Bioquant, 69120 Heidelberg, Germany \\ ${ }^{3}$ School of Physics and Astronomy, University of Edinburgh, EH9 3FD Edinburgh, UK \\ ${ }^{4}$ Institute of Molecular Biology, 55128 Mainz, Germany \\ ${ }^{5}$ Cologne Center for Genomics, University of Cologne, 50931 Cologne, Germany \\ ${ }^{6}$ Helmholtz Institute for Biomedical Engineering, RWTH Aachen University Medical School, 52074 \\ Aachen, Germany \\ ${ }^{7}$ Clinic I of Internal Medicine \& Center for Integrated Oncology, University Hospital Cologne, 50931 \\ Cologne, Germany \\ ${ }^{8}$ Interdisciplinary Centre for Clinical Research, RWTH Aachen University Medical School, 52062 \\ Aachen, Germany \\ ${ }^{9}$ Cologne Excellence Cluster on Cellular Stress Responses in Aging-Associated Diseases (CECAD), \\ University of Cologne, 50931 Cologne, Germany \\ ${ }^{10}$ These authors contributed equally to this work \\ ${ }^{11}$ Lead contact
}

\subsubsection{Abstract}

Processes like cell senescence are characterized by complex events giving rise to heterogeneous cell populations. However, the early molecular events driving this cascade remain elusive. We hypothesized that senescence entry is triggered by an early disruption of the cells' three-dimensional (3D) genome organization. To test this, we combined Hi-C, single-cell and population transcriptomics, imaging, and in silico modeling of three distinct cells types entering senescence. Genes involved in DNA conformation maintenance are suppressed upon senescence entry across all cell types. We show that nuclear depletion of the abundant HMGB2 protein occurs early on the path to senescence, and coincides with the dramatic spatial clustering of CTCF. Knocking-down HMGB2 suffices for senescence-induced CTCF clustering and for loop reshuffling, while ectopically-expressing HMGB2 rescues these effects. Our data suggest that an HMGB2-mediated genomic reorganization constitutes a primer for the ensuing senescent program. 


\subsubsection{Introduction}

Since the original description of in vitro replicative senescence (Hayflick, 1965), in vivo implications in development, wound healing, organismal ageing, and disease have been uncovered (van Deursen, 2014). In addition, clearance of senescent cells in mice was shown to improve health- and lifespan (de Keizer, 2017). Senescence entry is a result of integrated autocrine and paracrine signaling in the population (Acosta et al., 2013; Davalos et al., 2013; Hoare \& Narita, 2016) triggering replicative arrest, gene expression changes, secretory activity, and chromatin reorganization (Rai \& Adams, 2013). Telomere shortening (Herbig et al., 2004), reorganization of heterochromatin and the lamina (Narita et al., 2006; Sadaie et al., 2013; Shah et al., 2013; Swanson et al., 2013; Zhang et al., 2005), activation of transposable elements (De Cecco et al., 2013), or epigenetic changes on histones and the primary DNA sequence have been observed (Cruickshanks et al., 2013; Franzen et al., 2017; Neyret-Kahn et al., 2013). Still, despite some of these events sufficing for replicative arrest, early events signaling senescence entry remain elusive.

Thus, senescence is an attractive model for studying the structure-to-function relationship of chromosomes. This is now feasible due to the advent of Hi-C technology that captures spatial interactions within and between chromosomes (Belton et al., 2012). As a result of Hi-C studies, we now understand that chromosomes are partitioned into active A- and inactive B-compartments at the Mbpscale, and into consecutive topologically-associating domains (TADs) at the sub-Mbp scale. TADs harbor chromatin loops that tend to interact with one another more frequently than with loops in other TADs (Dixon et al., 2012). This higher-order organization is tightly linked to gene expression regulation, but also to cell cycle progression (Naumova et al., 2013).

For senescence, Hi-C has only been applied to fibroblasts undergoing oncogene-induced senescence (OIS) or maintained long-term in "deep" senescence, both constituting end-point states. In OIS, interaction loss within heterochromatin and lamin-associated regions was observed, and in a next step clustering of heterochromatic stretches to form senescence-associated heterochromatic foci (SAHFs; Chandra et al., 2015). In "deep" senescence, shorter-range interactions are favored over longerrange ones, thus compacting chromosome arms, while centromeres decondense (Criscione et al., 2016). Therefore, whether spatial genome reorganization triggers replicative senescence entry remains unaddressed.

We used three human primary cell types from individual donors - umbilical vein endothelial cells (HUVEC), fetal lung fibroblasts (IMR90), and mesenchymal stromal cells (MSC) - to identify a shared regulatory backbone instructing replicative arrest. We combined genomics, super-resolution microscopy and single-cell sequencing to discover that proteins of the high mobility group B (HMGB) family are implicated in regulating specific TAD boundaries, as well as in the spatial clustering of CTCF-bound chromatin. HMGBs are abundant nuclear proteins with characteristic HMG-box DNA- 
binding domains; they are known to distort DNA via unwinding, bending or looping (Stros, 2010). This renders them important for transcription, nucleosome remodeling, genome integrity, and recombination (Bonaldi et al., 2002; Laurent et al., 2010; Redmond et al., 2015; Lee et al., 2010; Little et al., 2013; Polanska et al., 2012). Critically, HMGBs are depleted from cell nuclei in senescent and ageing tissues (Abraham et al., 2013; Aird et al., 2016; Davalos et al., 2013; Ly et al., 2000; Taniguchi et al., 2009), and markedly overexpressed across cancer types (http://www.cbioportal.org/). Despite their physiological relevance, the binding and roles of HMGBs on chromatin remain enigmatic, but this work sheds new light onto their connection to the proliferative capacity, 3D genome folding, and transcriptional output of primary cells.

\subsubsection{Results}

\section{A shared regulatory backbone for senescence entry across cell types}

We hypothesized that different lineages share a common regulatory backbone controlling entry into replicative senescence. To test this, we obtained three distinct primary human cell types from individual donors/isolations: HUVEC (mesodermal) from three single donors, two isolates of IMR90 (endodermal), and MSC (multipotent) from five donors. As the path to senescence is characterized by heterogeneity (Smith \& Whitney, 1980), we used phenotypic and molecular markers to define the passage at which $\sim 65 \%$ of cells in each population had entered senescence. Cells staining positive for $\beta$-galactosidase, showing significantly reduced proliferation, and specific methylation changes at six senescence-predictive CpGs (Franzen et al., 2017) were deemed senescent (Figures 1A and S1A,B). Note here that one HUVEC and one MSC donor displayed a limited number of population doublings until senescence and, despite their convergent gene expression profiles, were excluded from further analyses. This highlights the idiosyncratic nature of cellular ageing due to donor-specific features.

Total RNA from proliferating and senescent populations from all cell types and donors was collected, depleted of rRNA, poly(A)+-enriched (except for HUVEC), and sequenced to >50 million read pairs each. Following mapping to the genome (hg19), read counts were normalized in silico to control for differences in transcript abundance as a result of senescence entry (Risso et al., 2014; Figure 3.1.S1C). Differential gene expression analysis revealed different numbers of up-/down-regulated genes in each cell type ( $\pm 0.6 \log 2$ fold-change), which nonetheless showed converging gene ontology (GO) profiles (Figure 3.1.S1D,E). 153 upregulated genes, mostly involved in cell growth, p53 responses, and ECM reorganization, were shared by all cell types (Figure 3.1.S1F). On the other hand, the 206 shared downregulated genes associated with cell cycle regulation, replication, DNA metabolism, but critically also with DNA conformation, chromatin organization, and DNA packaging (Figure 3.1.1B). The latter 
terms are due to genes like HMGB1/B2, TOP2A, NCAPD/-G/-H, or ASF1, and aligned with our hypothesis on 3D chromatin reorganization upon senescence entry.

We validated gene expression changes by comparison to available data from senescent IMR90 (Rai et al., 2014; Figure 3.1.S1G), and by time-course RT-qPCR on selected targets (Figure 3.1.S1H). We also isolated and analyzed nascent RNA from proliferative/senescent IMR90 via "factory" RNAseq (Melnik et al., 2016) to show that most regulation occurs at the level of transcription (Figures 1C and S1I). Due to their ability to bend DNA in vitro, their reduced expression in senescent and ageing tissue in vivo, and their unknown roles on chromatin, we focused on HMGB1/B2 and verified their suppression at the protein level in both IMR90 and HUVEC (Figure 3.1.1D). Single-cell mRNA profiling of senescent-entry cells

Transcriptional heterogeneity is inherent to senescent cell populations (Hernandez-Segura et al., 2017). To show that HMGB1/B2 are suppressed before known senescence markers appear, we performed single-cell mRNA sequencing by analyzing $\sim 8,300$ proliferating and $\sim 5,200$ senescent HUVEC on a 10X Genomics platform. Following multiplexed sequencing, we generated $\sim 30,000$ reads per cell in either state, and >2,500 individual transcripts were robustly captured per cell. Next, we clustered cells from each state based on their individual expression profiles. Unsupervised clustering showed proliferating and senescent cells segregating into four clusters (Figure 3.1.2A). Interestingly, the three major senescent-cell clusters reflected the relative distributions of HMGB2 and CDKN1A (p21) expression: low HMGB2 levels mostly marked cells with medium-to-high CDKN1A levels (i.e. replicatively-arrested cells), yet >1,800 cells carried little HMGB2 mRNA without CDKN1A induction. Similarly, >2,500 proliferating cells showed robust HMGB1 but already low HMGB2 levels (Figure 3.1.2B,C). Senescent cells lacking HMGB2 expression were also marked by high levels of the IL6 and IL8 SASP genes (Figure 3.1.2B). Importantly, the gene expression profiles previously reported to hold key roles in senescence and the cell cycle, like LMNB1, EZH2, PCNA or CCNA2 also differentially marked proliferating and senescent subpopulations, but were less informative for clustering (Figure 3.1.2B).

Last, we exploited the heterogeneity in HUVEC populations to perform pseudo-time course analyses of scRNA-seq data (Haghverdi et al., 2016). We found that the gradual drop in HMGB1/B2 levels in proliferating HUVEC sufficed for describing 7 states that appear consecutively derived from one another (Figure 3.1.2D). On the other hand, the 8 senescent cell-states along the main path display variable amounts of HMGB1/B2, and are best described by a rise in CDKN1A levels and by strong IL8 and CXCL1 SASP-gene expression. Still, subclusters 1 and 4 had HMGB2 suppressed, but HMGB1 robustly detected (Figure 3.1.2D). This, to our knowledge, first ever transcriptome of single replicatively-senescent cells documents how HMGB2 suppression is an early event marking senescence entry. 

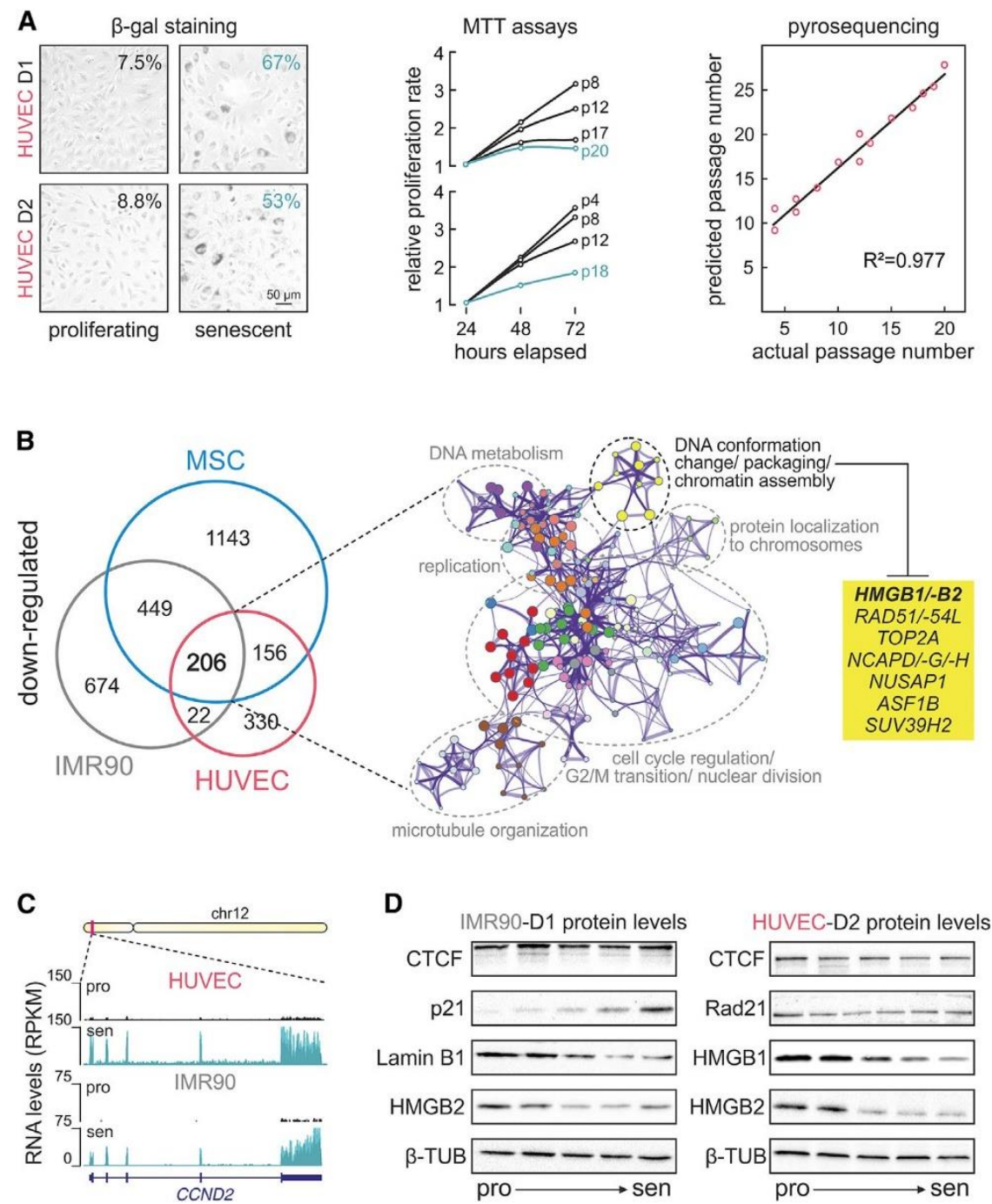

Figure 3.1.1. Senescence entry signals suppression of specific pathways.

(A) Left: $\beta$-galactosidase staining of proliferating and senescent HUVEC donors D1 and D2; percent of $\beta$-gal positive cells are indicated. Middle: MTT assays for D1 and D2 at different passages; cells were deemed senescent at passage 20 and 18, respectively (green lines). Right: Spearman's correlation (R2) between actual and predicted HUVEC passage based on the methylation at six CpGs.

(B) Left: Genes downregulated in senescence across cells types. Right: Pathways associated with shared downregulated genes. Nodes (yellow) containing genes involved in DNA conformation, DNA packaging or chromatin assembly are highlighted, and selected genes are listed (box).

(C) CCND2 locus RNA-seq profiles in HUVEC and IMR90.

(D) HMGB1, HMGB2, CTCF, Lamin B1, Rad21, and p21 western blots at five different passages in HUVEC and IMR90; $\beta$-tubulin provides a control. 


\section{HMGB2 depletion precedes p21 activation and induces heterochromatic changes}

To complement scRNA-seq data and examine changes in HMGB1/B2 titers, we turned to single-cell immunofluorescence. Super-resolution imaging found the abundant HMGB1/B2 proteins localizing in non-DAPI-dense nuclear areas (Figure 3.1.S2A). Widefield microscopy coupled to semiautomated quantification of fluorescence levels (see STAR Methods) revealed that senescent nuclei almost doubled in area across cell types, and larger nuclei were almost invariably $\beta$-galactosidase positive (Figure 3.1.3A,B). This urged us to stratify all microscopy data according to increasing nuclear sizes. This way we could verify that HMGB1/B2 were depleted from essentially all large HUVEC, IMR90, or MSC nuclei (Figure 3.1.3C). Although HMGB1/B2 nuclear depletion appeared coordinated amongst individual cells $(\rho=0.81)$, p21 upregulation - a hallmark of replicative arrest - correlated rather poorly with low HMGB2 titers ( $\rho=0.35$; Figure 3.1.3D,E). This agrees with our scRNA-seq data where HUVEC with low HMGB2 titers and no CDKN1A and/or robust HMGB1 levels represent an early state on the path to senescence.

Focusing on size-discretized nuclei, we found that larger and HMGB2-depleted nuclei show specific decrease in H3K27me3 levels, the mark characteristic of facultative heterochromatin (see Shah et al., 2013), while also displaying increased HP1 $\alpha$ levels (marking constitutive heterochromatin, but without SAHF formation as in OIS; Narita et al., 2006; Figure 3.1.S2B). This was concomitant with a drop in histone acetylation measured using H3K27ac or H4K16ac as markers (Figure 3.1.S2C,D). Finally, increased H3K9-methylation was confirmed in senescent HUVEC and IMR90 via ELISA (Figure 3.1.S2E), while the senescent-induced drop in $\mathrm{H} 3 \mathrm{~K} 27 \mathrm{ac} / \mathrm{me} 3$ was in line with changes in H3K27ac/me3 ChIP-seq signal (Figure 3.1.S2F,G).

\section{Senescence entry is marked by changes in 3D chromatin folding}

Population and single-cell data encouraged us to interrogate changes in whole-genome 3D folding upon senescence entry via Hi-C. We used HindIII and obtained >300 million read pairs per donor and condition, after sequencing two technical replicates from two HUVEC and IMR90 donors/isolates from, and one MSC donor (Figure 3.1.S3A). After confirming reproducibility between Hi-C maps from the same cell type and condition (Figure 3.1.S3B), we merged replicates and could now afford $20-\mathrm{kbp}$ resolution of spatial chromatin interactions in both proliferating and senescent cells (higher than that afforded for "deep" senescence; Criscione et al., 2016). Initially, we looked into interchromosomal interaction changes between whole chromosomes upon senescence entry; both HUVEC and IMR90 chromosomes mostly displayed decreased, cell type-specific, trans-interactions (Figure 3.1.S3C).

We next examined intrachromosomal interactions; two features stand out when comparing Hi$\mathrm{C}$ interaction maps from proliferating and senescent HUVEC. First, stronger longer-range interactions 
emerge upon senescence entry; second, apparent insulation, especially between large higher-order domains, is seen in senescent cells (Figures 3.1.4A and 3.1.S4A). This holds true across all cell types and chromosomes studied (Figure 3.1.S3D and S4A-C). Second, interaction changes between single donors/isolates visualized at a $200 \mathrm{kbp}$-resolution are almost invariably of the same nature (Figure 3.1.S3E). But, when different cell types are compared, the same chromosomal regions refold in a cell type-specific manner upon senescence (Figures 3.1.4B and 3.1.S4F), and this is in line with senescence epigenetically reprogramming each cell type.

In addition, most $\mathrm{Hi}-\mathrm{C}$ data to date display more changes at the level of $\mathrm{A} / \mathrm{B}$ compartments than at that of TADs (e.g. Criscione et al., 2016; Dixon et al., 2012). However, we observed limited switching between $\mathrm{A} / \mathrm{B}$ compartments (Figures 3.1.4B and 3.1.S5A). We went on to define TADs in our Hi-C data from both conditions across cell types. We employed "TADtool" (Kruse et al., 2016) and the resolution afforded by our lowest-covered dataset (40-kbp resolution in MSC), while applying similar TAD-calling parameters for proliferating or senescent data in each cell type. This analysis returned a total of 3,000 TADs for HUVEC and IMR90, and 3,500 for MSC in either state. In IMR90 and MSC, $\sim 50 \%$ of TADs remain unchanged upon senescence entry, while $\sim 20 \%$ shift at least one of their boundaries by $\geq 80 \mathrm{kbp}$ and $\sim 25 \%$ fuse into larger TADs (Figure 3.1.4C). TAD changes were more drastic in HUVEC, with 24\% TADs shifting boundaries, and 43\% fusing into larger ones - in agreement with the strong differences seen in HUVEC Hi-C (Figure 3.1.S4A-C). Interaction decay plots revealed widespread changes in both longer- and shorter-range interactions upon senescence entry (Figures 3.1.4D and 3.1.S5B), which differs from what was observed for "deep" senescence (Criscione et al., 2016; Figure 3.1.S5C,D).

Finally, we used 3D-DNA FISH (Roukos et al., 2015), with probes targeting three increasingly separated regions on the long arm of chr12 to query conformational changes. In senescent HUVEC, which are predominantly tetraploid (Figure 3.1.S6A), FISH showed all probes becoming more separated from one another upon senescence and assuming less peripheral positioning in the larger senescent nuclei (Figure 3.1.S6B-E). In IMR90, which remain diploid, the two most distant probes come significantly closer together upon senescence, while the intervening one separates out, and only one probe pair shows less peripheral positioning (Figure 3.1.S9A-E). Critically, in both HUVEC and IMR90 the FISH data agree with changes seen by Hi-C (i.e. increased probe separation matched by decreaseinteraction frequency; Figure 3.1.S6C,E). 

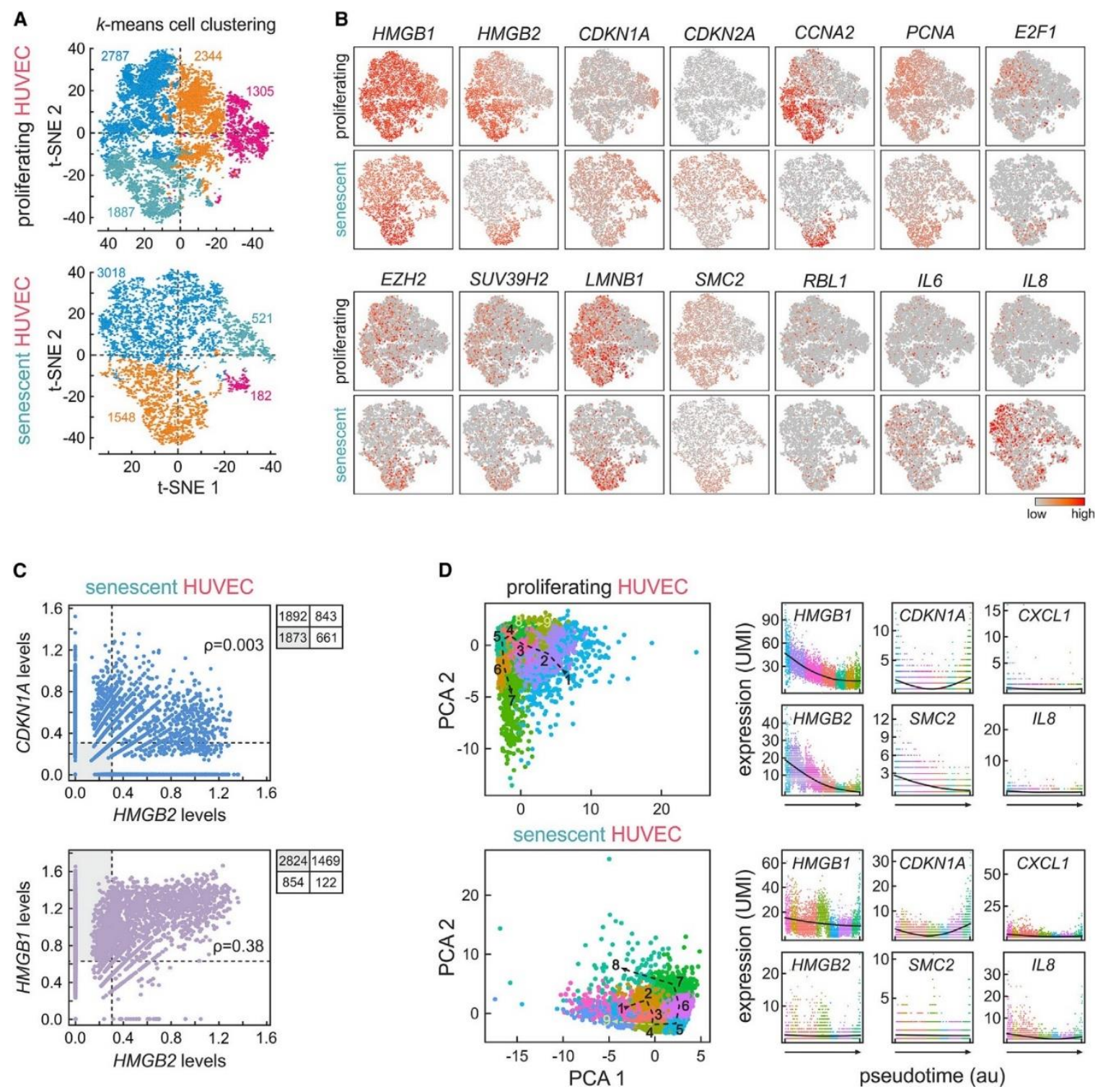

Figure 3.1.2. scRNA-seq highlights early HMGB2 suppression.

(A) Unsupervised clustering of scRNA-seq data from proliferating or senescent HUVEC. Each dot represents one cell, and numbers of cells per cluster is indicated.

(B) mRNA levels of 14 senescence-regulated genes in proliferating or senescent cells.

(C) HMGB2 scRNA-seq read counts were plotted against HMGB1 and CDKN1A read counts; median values minus one S.D. (dotted lines), Pearson's correlation coefficients ( $\rho$ ), and the number of cells in each quadrant are indicated.

(D) Left: Pseudo-time course clustering of scRNA-seq data from proliferating or senescent HUVEC. The main path (black line) also breaks into a secondary leg (yellow line). Right: Expression of 6 key genes in the clusters along the main path of proliferating (top) or senescent cells (bottom). 
Results
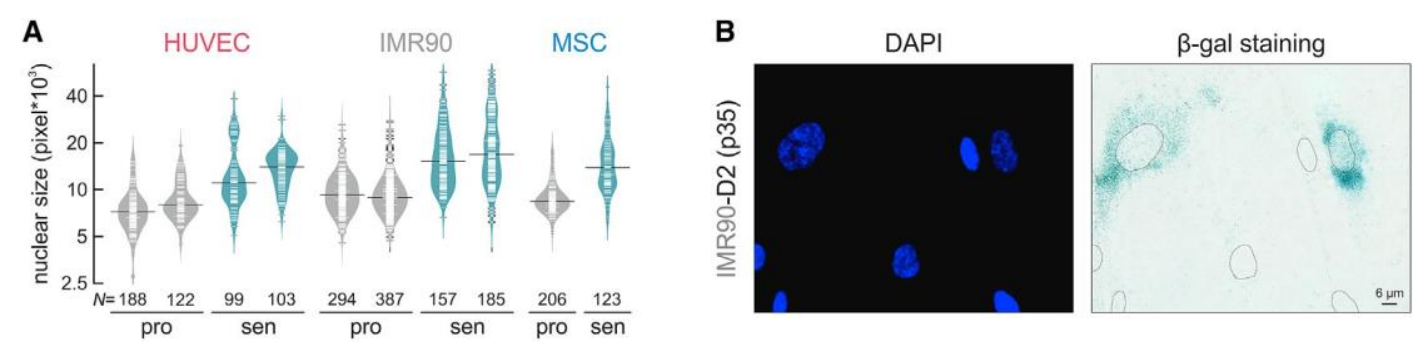

C
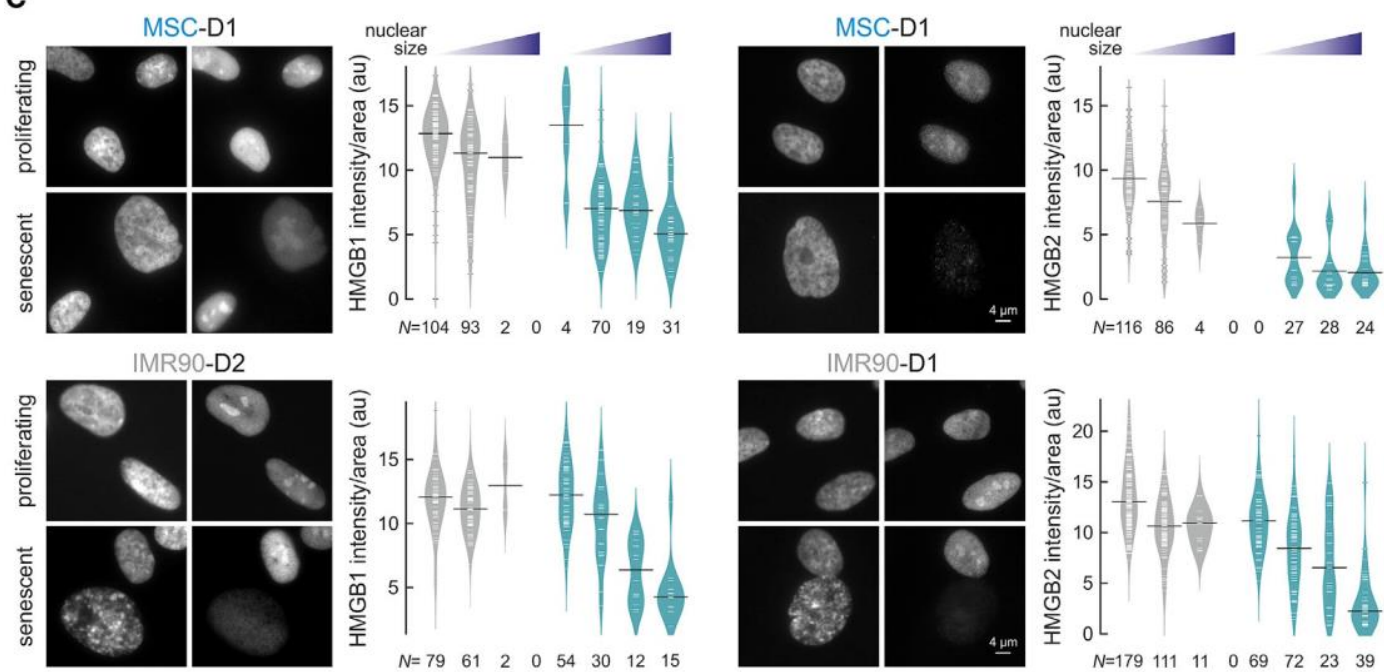

HUVEC-D2
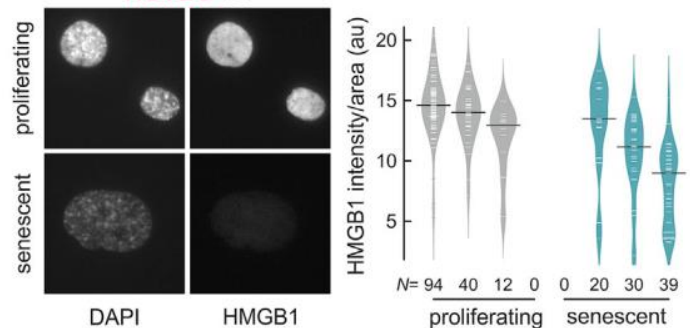

HUVEC-D2

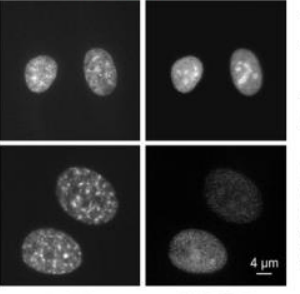

DAPI
$N=179 \begin{array}{lllllll}111 & 11 & 0 & 69 & 72 & 23 & 39\end{array}$

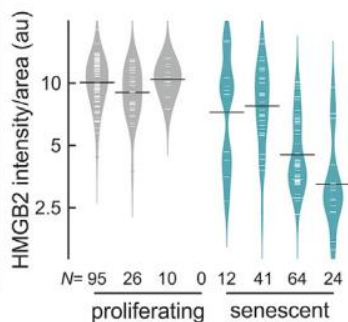

D
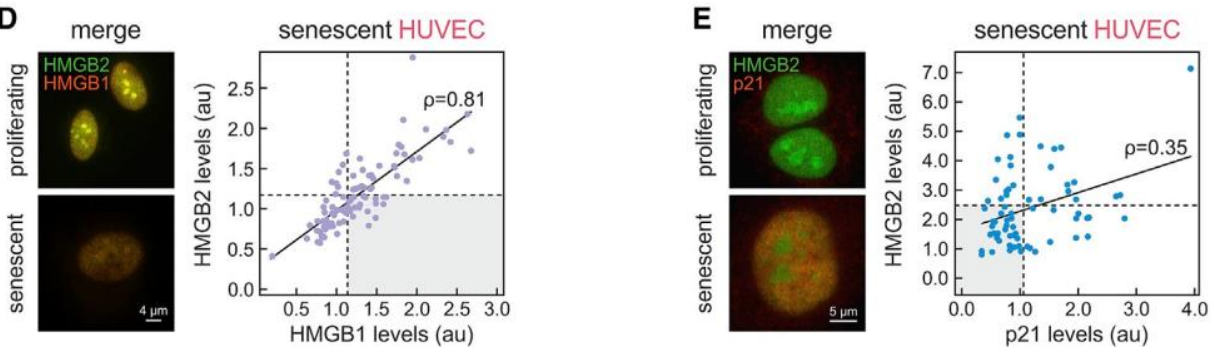

Figure 3.1.3. Senescence entry is characterized by HMGB2 nuclear loss.

(A) Distribution of nuclear sizes in proliferating (grey) or senescent (green) HUVEC, IMR90, and MSC. The total number of nuclei measured $(\mathrm{N})$ is indicated.

(B) Typical images of senescent IMR90 stained for $\beta$-galactosidase and DAPI.

(C) Typical images of proliferating/senescent MSC, IMR90, and HUVEC immunostained for HMGB1/B2 (left). Bean plots (right) show changes in HMGB1/B2 levels in cells grouped according to increasing nuclear size. The total number of nuclei measured $(\mathrm{N})$. is indicated.

(D) Typical images of proliferating/senescent HUVEC immunostained for HMGB1/B2. Fluorescence levels were used to produce scatter plots; median values minus one S.D. (dashed lines) and Pearson's correlation coefficients ( $\rho)$ are indicated.

(E) As in panel D, but for HMGB2 and p21. 


\section{Heterochromatic remodeling does not adequately explain 3D genome reorganization}

Genome reorganization in OIS and in "deep" senescence involves formation of characteristic SAHFs (Chandra et al., 2015) and strong chromatin compaction, respectively (Criscione et al., 2016). Here, senescence entry coincides with a heterochromatic shift (Figure 3.1.S2B), and we asked whether this suffices to explain the changes seen by Hi-C (Figure 3.1.S4). As it is not straightforward to experimentally decouple HMGB1/B2 loss from heterochromatic changes, we used molecular dynamics simulations of 3D chromatin folding. This in silico approach models preferential low-attraction interactions of loci on the basis of their chromatin identity (defined using ENCODE ChIP-seq; see STAR Methods) that faithfully recapitulate Hi-C interactions (Brackley et al., 2016a). We focused on a 15Mbp region on the long arm of chr12. Based on our ChIP-seq data, heterochromatic positions were kept the same for both states modelled, but H3K9me3-mediated interaction strength was increased by 30\% in the senescent model (consistent with Figure 3.1.S2B-G). Following multiple iterations, average interaction profiles only partly recapitulated experimental Hi-C profiles (Figure 3.1.S6F,G). Thus, as this strengthened heterochromatic bridging did not sufficiently explain all changes occurring upon senescence entry (Figure 3.1.S6H), we went on to explore a potential contribution of HMGB1/B2 in chromatin folding.

\section{HMGB1 and HMGB2 bind non-overlapping active chromatin sites}

Our understanding of the roles of HMGB1/B2 remains incomplete due to the fact that formaldehyde crosslinking fails to capture HMG-box factors bound to chromatin (Pallier et al., 2003; Redmond et al., 2015; Teves et al., 2016). To circumvent this, we devised a dual-crosslinking approach for HMGB1/B2 ChIP-seq (see STAR Methods) and identified 1574 and 1188 robust HMGB2 peaks in proliferating HUVEC and IMR90, respectively; we also identified 391 peaks in senescent IMR90 (Figures 3.1.5A and 3.1.S7A). HMGB2 mostly binds promoters or bodies of active genes associated with processes relevant to senescence (e.g. ECM organization, wound healing or mitotic phase transition; Figures 3.1.5B and 3.1.S7B). Notably, at least twice as many genes bound by HMGB2 are up- rather than downregulated (Figure 3.1.5C). As HUVEC and IMR90 deploy different gene expression programs and HMGB2 predominantly associates with active loci, the two cell types only share 131 ( 10\%) HMGB2 peaks. Still, there are eight-fold more up- than downregulated genes associated with these 131 peaks, and they are linked to such processes as cell proliferation and cell ageing (Figure 3.1.5C).

Of the 391 HMGB2 peaks found in the senescent IMR90 population, 220 were at positions also bound in proliferative cells (and are thus rather the result of heterogeneity than of persistent binding), whereas 171 seem to form de novo. These 220 peaks associated with mitotic processes and mRNA surveillance (Figure 3.1.5D). Critically, none of the 171 senescence-specific peaks overlapped SASP 
genes as was proposed for OIS (Aird et al., 2016; Figures S7D). Thus, remodeling of HMGB2-binding in replicative senescence does not involve SASP loci.

Next, to identify transcription factors (TFs) potentially co-bound with HMGB2, we analyzed DNase I-hypersensitive footprints under HMGB2 ChIP-seq peaks for known TF motif enrichment. Following filtering for well-expressed TFs, this analysis returned a number of candidate TFs that included architectural ones, like CTCF, relevant to cell cycle regulation, like E2F-family members and FOXP1/2, or involved in proinflammatory signaling, like NFKB2 and RELA (Figure 3.1.S7E). These motifs are enriched under HMGB2 peaks from proliferative cells, which implies that loss of HMGB1/B2 from these positions might either dismantle co-bound complexes (e.g. in the case of E2F factors to hinder cell cycle progression) or facilitate assembly of new ones (e.g. in the case of proinflammatory SASPrelated TFs that remain largely inactive in proliferating cells).

Finally, using the same dual-crosslinking approach, we generated HMGB1 ChIP-seq profiles in proliferating HUVEC and IMR90. We identified 810 and 593 HMGB1 peaks, respectively, more than $30 \%$ of which were intergenic, and rarely overlapped HMGB2-bound sites, suggesting that the two factors have non-overlapping functions (Figure 3.1.S7F,G).

\section{Chromatin-bound HMGB2 marks a subset of TAD boundaries}

Using high-resolution Hi-C data from IMR90 (Rao et al., 2014), we found that HMGB2 ChIPseq peaks display good correlation to positions of interaction insulation (i.e. at TAD/sub-TAD boundaries; Figure 3.1.S8A). Correlation of HMGB2 peaks to TAD extremities from proliferating HUVEC and IMR90 showed that they mark TAD boundaries comparably to CTCF, and this demarcation markedly weakened for TADs from senescent cells (Figure 3.1.5E). This implies that TAD-boundary changes upon senescence entry (see Figure 3.1.4F) are linked to HMGB2 depletion from nuclei. In more detail, HMGB2 marks TADs of proliferating cells that both shift their boundaries or remain unchanged in senescence - but not of those that fuse. Intriguingly, non-changing TADs have both boundaries marked by HMGB2, whereas those that shift a boundary display reciprocal demarcation with HMGB2 enriched on one side and CTCF on the other (Figures 5F and S8B). Also, TADs that lose HMGB2 from their boundaries harbor almost twice as many up- than down-regulated genes compared to other TAD subgroups, in line with our ChIP-seq data (Figure 3.1.5C,G). Approx. 10\% of HMGB2 peaks fell within $40 \mathrm{kbp}$ of a TAD boundary in proliferating cells, which is significantly more than what would be expected by chance ( $\mathrm{P}<0.001$, Fisher's exact test). In order to link HMGB2 boundarypositioning to spatial reorganization upon senescence entry, we looked at average Hi-C interaction profiles around HMGB2 peaks. Interaction insulation at HMGB2-bound boundaries collapsed when comparing senescent cells to proliferating cells, and this effect was far less pronounced when considering all peaks (Figure 3.1.5H). Of course, since our HUVEC Hi-C afforded higher resolution, we repeated all analyses using 20-kbp resolution data. At this resolution, more TAD changes arose

(Figure 3.1.S8C), more HMB2 peaks resided at TAD boundaries ( 16\%; Figure 3.1.S8D), but still 
demarcation of TAD subgroups, gene expression changes therein, and changes around HMGB2-bound boundaries were of the same nature (Figure 3.1.S8E-G).

A
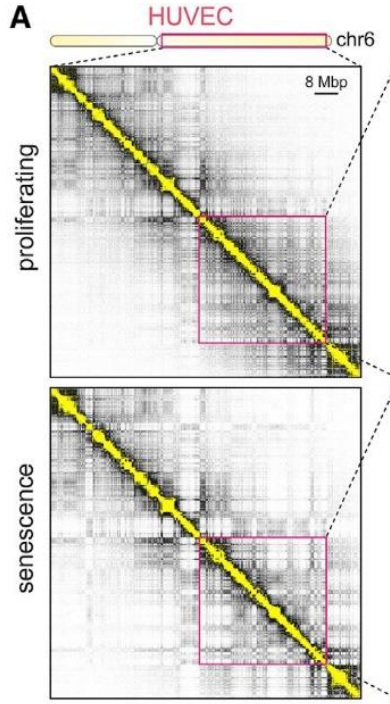

100-kbp resolution

B
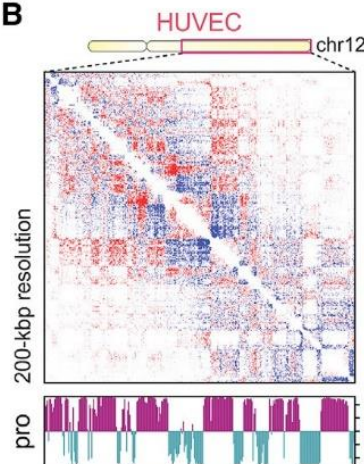

๖్

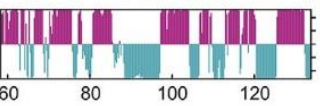

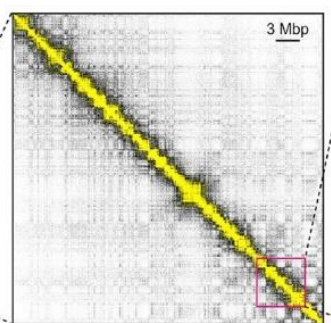
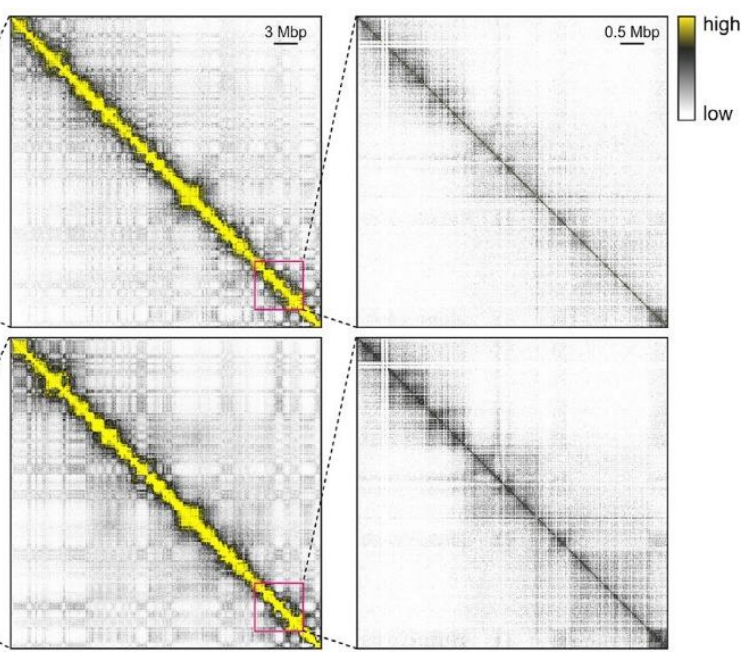

40-kbp resolution

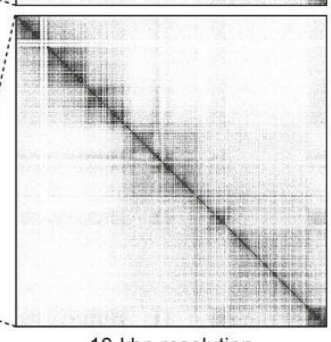

10-kbp resolution

MSC
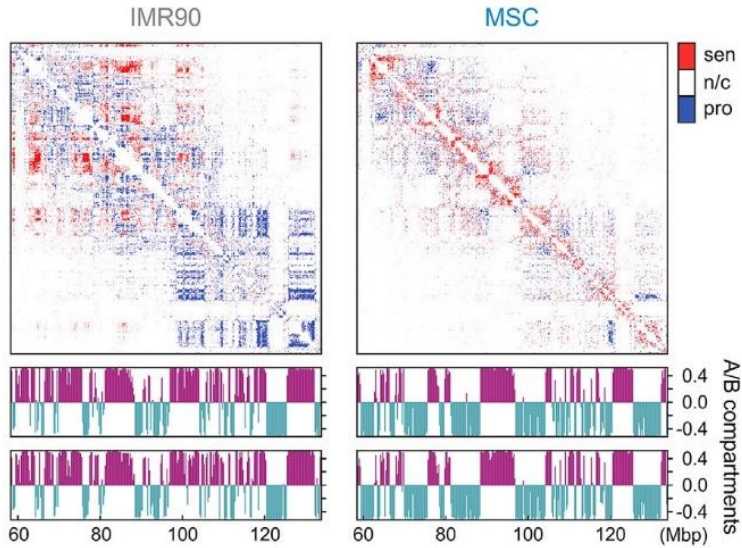

C

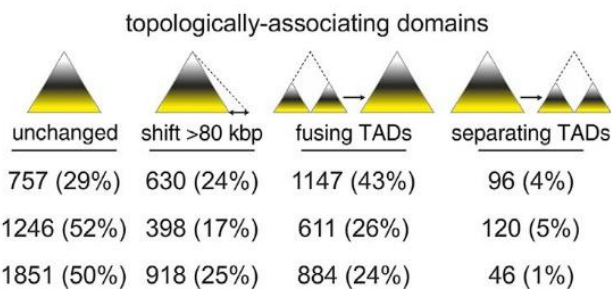

D HUVEC chr21

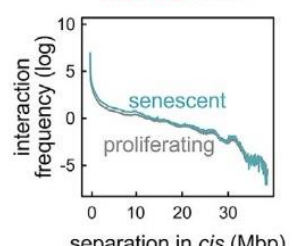

Figure 3.1.4. Senescence entry is accompanied by 3D genome reorganization.

(A) $\mathrm{Hi}-\mathrm{C}$ contact frequencies along the long arm of chr6 from proliferating and senescent HUVEC at increasing resolution.

(B) Binary increase/decrease in Hi-C contact frequencies between senescent and proliferating HUVEC, IMR90, or MSC along a subregion of chr12. Aligned below are graphs showing A- (purple) and B-compartments (green) for each cell type and condition.

(C) The number of TADs (percentages in brackets) that remain unchanged, shift one boundary, fuse into one larger TAD, or separate into smaller TADs upon senescence are listed for all cell types.

(D) Interaction frequency decay as a function of cis-separation between fragments for chr21 in proliferating or senescent HUVEC Hi-C data. 
A

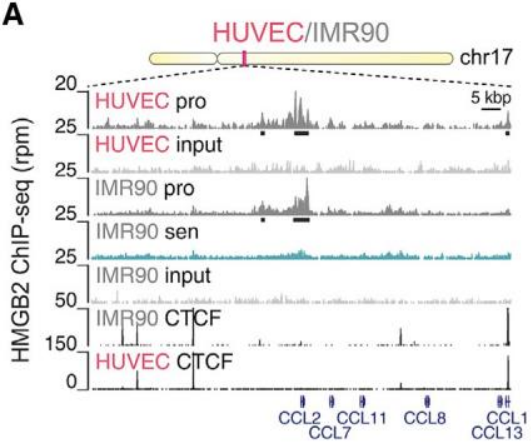

c

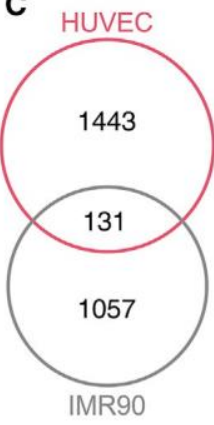

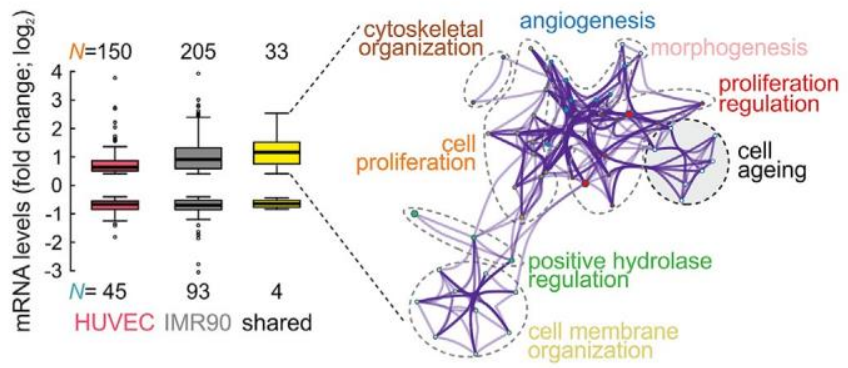

B

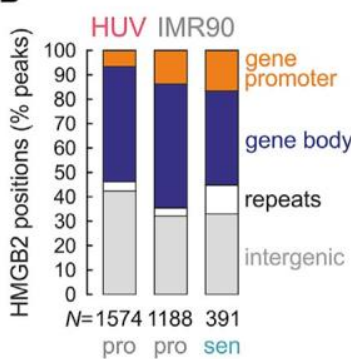

E

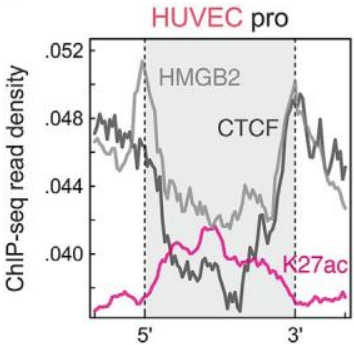

$\mathbf{F}$

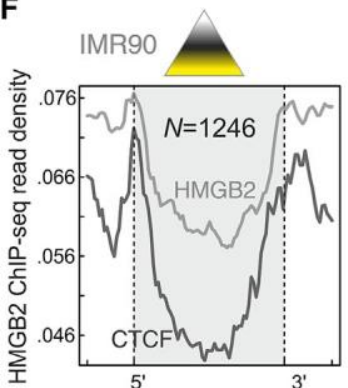

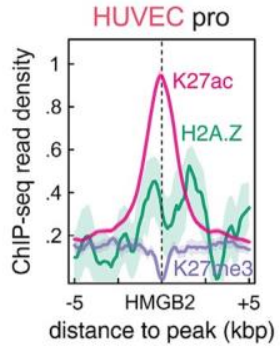

IMR90 pro
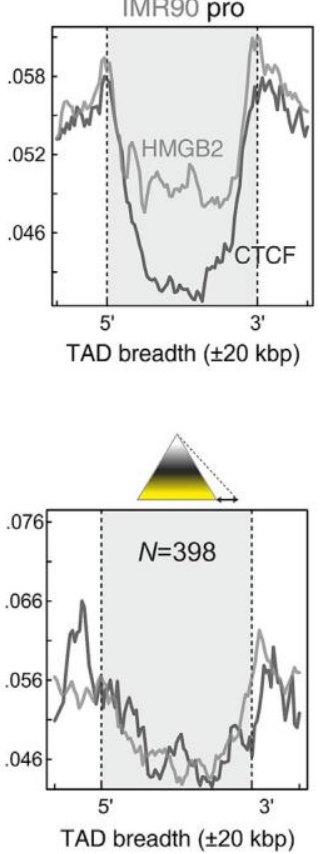

D
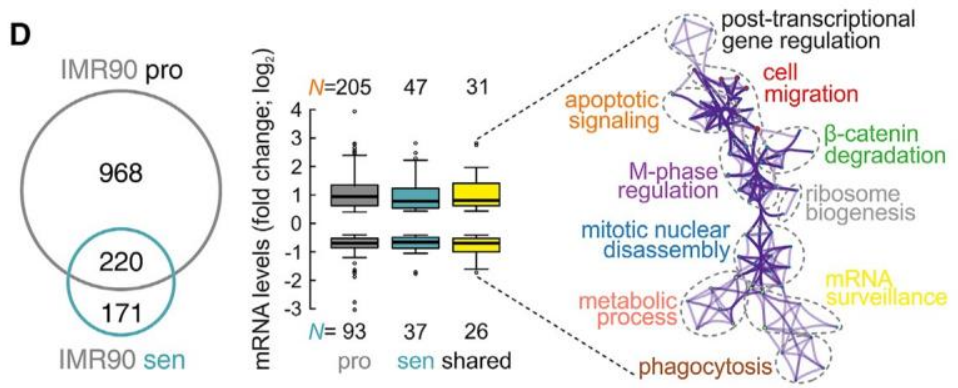

G
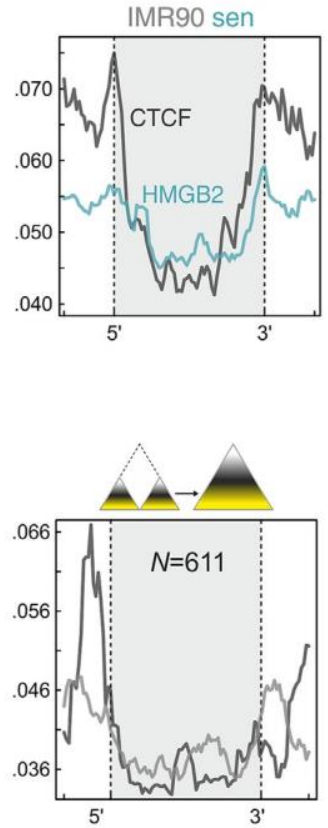

IMR90 TADs
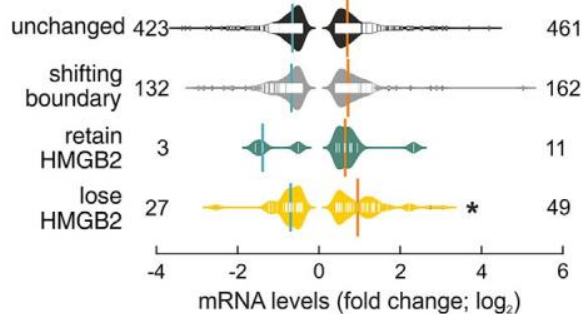

H

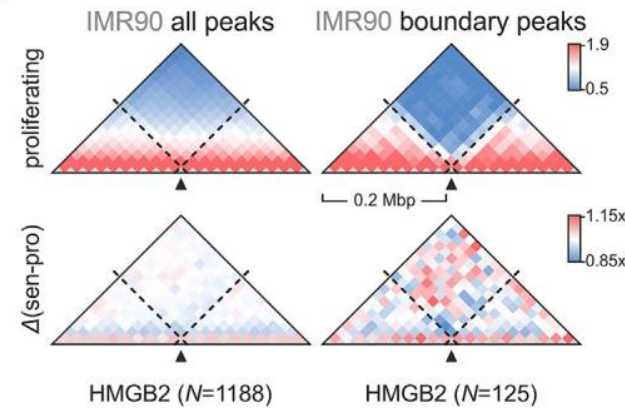

Figure 3.1.5. HMGB2 chromatin-binding properties.

(A) HMGB2 ChIP-seq from proliferating/senescent HUVEC and IMR90 along a chr17 locus; CTCF ChIP-seq and input profiles are aligned below.

(B) Left: Percentage of HMGB2 peaks from proliferating/senescent HUVEC and IMR90 overlapping promoters, gene bodies, repeats, or intergenic space. The total number of peaks $(\mathrm{N})$ per dataset is indicated. Right: Distribution of H3K27ac, H3K27me3, and H2A.Z ChIP-seq signal in the $10 \mathrm{kbp}$ around HUVEC HMGB2 peaks.

(C) Left: HMGB2 peaks shared between HUVEC and IMR90. Middle: Log2 fold changes of differentiallyexpressed genes bound by HMGB2. The total number of genes $(\mathrm{N})$ is indicated. Right: GO terms associated with HMGB2-bound upregulated genes in both HUVEC and IMR90.

(D) As in panel C, but for HMGB2 peaks shared between proliferating and senescent IMR90.

(E) Distribution of HMGB2 ChIP-seq signal along TADs ( $\pm 20 \mathrm{kbp})$ in proliferating/senescent HUVEC or IMR90; CTCF and H3K27ac distributions serve as controls.

(F) As in panel E, but for IMR90 TADs that do not change (left), shift one boundary (middle), or fuse into one larger TAD (right). The total number of TADs in each group $(\mathrm{N})$ is indicated. 
(G) $\log 2$ fold changes of differentially-expressed genes located within the different TAD subsets. The total number of genes in each group $(\mathrm{N})$ is indicated. *significantly different to "unchanged"; $\mathrm{P}<0.01$, Wilcoxon-MannWhitney test.

(H) Normalized Hi-C interaction frequencies (top). or senescence-induced changes Hi-C interactions (bottom). in the $0.4 \mathrm{Mbp}$ around all or TAD-boundary HMGB2 peaks from IMR90.

\section{HMGB2 knockdown affects heterochromatin and induces spatial CTCF clustering}

The above analyses are support a role for HMGB2 in reorganizing chromatin topological upon senescence entry. To functionally establish this, we knocked-down HMGB2 in HUVEC and IMR90 using self-delivering siRNAs (as primary cells are difficult to transfect and lipofectamine stresses cells and convolutes their phenotypes). This approach strongly depleted the HMGB2 protein (Figure 3.1.S9A), but unlike previously reported effects for sustained HMGB1 knockdown (Davalos et al., 2013), the loss of HMGB2 only caused a mild increase in the number of senescent cells in the 72-hour treatment window, while also marginally affecting nuclear size, DNA replication, or gene expression (Figure 3.1.S9B-D). However, after labeling nascent transcripts in live cells via a short (5-min) EUTP pulse and quantifying EU-RNA fluorescence, we uncovered a $~ 30 \%$ drop in nascent RNA levels upon HMGB2 knockdown; a similar decrease of $\sim 10 \%$ in nascent RNA production was seen in senescent IMR90 (Figure 3.1.6A). Also, knocking-down HMGB2 sufficed for inducing HP1 $\alpha$ and suppressing H3K27me3 levels (Figures 3.1.6B and 3.1.S9E), thus recapitulating the shift between constitutive and facultative heterochromatin seen in senescence. These effects highlight that HMGB2 depletion is an upstream event affecting both the organization of the genome and its functional output.

Next, we asked whether HMGB2 depletion also affected the levels and distribution of CTCF in primary cells. Super-resolution imaging revealed that in proliferating HUVEC HMGB2 and CTCF foci are tightly alternating and interwoven. Upon senescence entry, CTCF is dramatically reorganized in large senescence-induced CTCF clusters (SICCs) devoid of HMGB2 signal (Figure 3.1.6C). SICC formation was consistently found in senescent HUVEC and IMR90, and HMGB2 knockdown phenocopied this despite the smaller nuclear sizes in knockdown-cells (Figures 3.1.6D and 3.1.S9FH). Notably, SICCs did not overlap HP1 $\alpha$ foci (Figure 3.1.S9I), thus bear no resemblance to SAHFs and are specific to replicative senescence. SICC formation was not accompanied by strong cohesin reorganization in senescent IMR90, despite extensive colocalization of CTCF and cohesin on chromatin (Rao et al., 2014). Still, decreased cohesin levels in SICC-positive cells were recorded (Figure 3.1.S9J). Perhaps surprisingly, <200 HMGB2 peaks from HUVEC or IMR90 were proximal to CTCF peaks, and co-immunoprecipitation of either CTCF or HMGB2 did not reveal physical interaction between the two factors (Figure 3.1.S9K-M). In addition, CTCF binding at both standalone and HMGB2-proximal sites remains unchanged between proliferating and senescent cells (Figure 3.1.S9N).

To assess the impact of HMGB2 depletion on whole-genome folding, we applied Hi-C to control and HMGB2-knockdown HUVEC. This data revealed apparent differences between the two states, but, just like in senescence, only limited A/B-compartment remodeling. At 40-kbp resolution, 
interactions around HMGB2 peaks were particularly disrupted (Figures 3.1.6E and 3.1.S10A). Changes to TAD boundaries mirrored precisely those seen in senescent HUVEC (Figures 3.1.6F) and average interaction profiles around HMGB2- and/or CTCF-bound positions again recapitulated the effects of senescence entry at TAD boundaries (Figure 3.1.S10B,C).

\section{Towards a molecular mechanism for SICC formation}

Closer inspection of HMGB2 ChIP-seq aligned to high-resolution HUVEC Hi-C (Rao et al., 2014) revealed loop-like interactions between multiple HMGB2 peaks (Figure 3.1.7A). Thus, we inquired whether HMGB2 forms loops similar to CTCF (Rao et al., 2014). We interrogated all pairwise cis-interactions involving HMGB2 peaks looking for local Hi-C signal maxima exceeding background. We found that $\sim 2,900$ and $~ 5,000$ loop structures emanating from HMGB2-bound positions in IMR90 and HUVEC, respectively, with their average profiles and intensities being directly comparable to those of CTCF loops (Figure 3.1.7B).

As heterochromatic changes did not fully explain conformation changes observed by Hi-C, we revisited modeling of the 15-Mbp region on chr12. Typically, HMGB2 peaks are positioned between CTCF loops (Figures 3.1.7A,C) and we hypothesized they might act to insulate further CTCF-CTCF interactions. To model this mode-of-action, we devised three in silico scenarios. First, in proliferating cells stable looping between convergent CTCF-bound sites was allowed (based on IMR90 loops; Rao et al., 2014), but loops across HMGB2 peaks were disallowed. Second, in "senescence model I" insulation by HMGB2 was alleviated to mimic its depletion from nuclei. Third, in "senescence model II", additional stochastic CTCF-CTCF interactions are favoured upon HMGB2 depletion. All three scenarios were implemented on top of the framework used before (Figure 3.1.S6F-H). Each scenario was modeled for 48 iterations, and generated increasingly stronger clustering of CTCF-bound chromatin in the absence of HMGB2 (Figure 3.1.7C,D).

To validate these models experimentally, we used HiChIP that allows spatial interactions owing to a particular TF to be subselected and interrogated independently of all others (Mumbach et al., 2016). We pulled-down CTCF and were able to detect $\sim 2,000$ and $\sim 1,000$ robust CTCF-CTCF loops per condition in HUVEC and IMR90, respectively (despite lower complexity in CTCF-HiChIP compared to Hi-C libraries; Figure 3.1.S10D). Comparing intra-chromosomal loops between proliferating and senescent cells revealed increased loop lengths, with $\sim 40 \%$ being unique to each state (Figure 3.1.S10E). Inspection of multi-Mbp genomic stretches populated by CTCF-CTCF loops, new longrange CTCF loops develop around positions where HMGB2 binding is lost, just as our in silico models predicted. On the other hand, around positions where HMGB2 binding persists or only occurs in senescence, local loop depletion was observed (Figures 3.1.7E and 3.1.S10F). Hence, HMGB2 peaks indeed function as "insulators" for flanking CTCF-bound sites. However, we ruled out the possibility of CTCF binding at HMGB2 sites that contain motifs resembling those cognate for CTCF by assessing its binding at these "neoCTCF" sites in senescent and HMGB2-knockdown cells (Figure 3.1.7F). 
Importantly, in both HUVEC and IMR90, genes within $10 \mathrm{kbp}$ of CTCF loop anchors that reshuffle upon senescence entry display significantly stronger expression changes compared to genes within 10 kbp of static loops (Figure 3.1.S10G).

Ultimately, to unambiguously link HMGB2 loss to SICC formation, we generated IMR90 populations carrying either a doxycycline-inducible HMGB2-GFP fusion or GFP alone. The constructs were inserted into the genome using a piggybac-based vector. We selected, expanded, and drove cells into senescent heterogeneous populations (to average out clonal variation). HMGB2 overexpression was induced in senescent cells using doxycycline for $24 \mathrm{~h} ; \sim 50 \%$ of cells in each population expressed GFP or the HMGB2-GFP fusion (Figure 3.1.7G). Of those carrying only GFP, $>45 \%$ showed strong SICC formation, while significantly fewer $(<20 \%)$ of those expressing HMGB2-GFP displayed SICCs (Figure 3.1.7G). Thus, the nuclear presence or absence of HMGB2 is sufficient and instructive for induction of CTCF clusters upon senescence entry.

\section{Cancer cell proliferation potency relies on HMGB factors}

HMGB2 proteins are depleted from cell nuclei upon senescence entry and this depletion reduces nascent RNA levels globally (Figure 3.1.6A). Conversely, in tumour cells enhanced proliferative capacity is accompanied by increased global transcription (Kotsantis et al., 2016). Importantly, unlike senescent cells, a large variety of cancer cells display HMGB2 overexpression (Figure 3.1.7H). We hypothesized that these higher titers might explain their proliferative potency. As proof-of-principle, we used two lung lines for HMGB2 knockdown, and could show that suppressing HMGB2 affects genes like HDAC9, RBL1, and TP53 (upregulated), or LMNB1, EZH2, and SUV39H2 (downregulated), but not HMGB1 (Figure 3.1.7I). Importantly, this resulted in severe growth arrest of adenocarcinoma cells (Figure 3.1.7J), highlighting the potential role of HMGB2 in the maintenance of the proliferative state in cancer. 

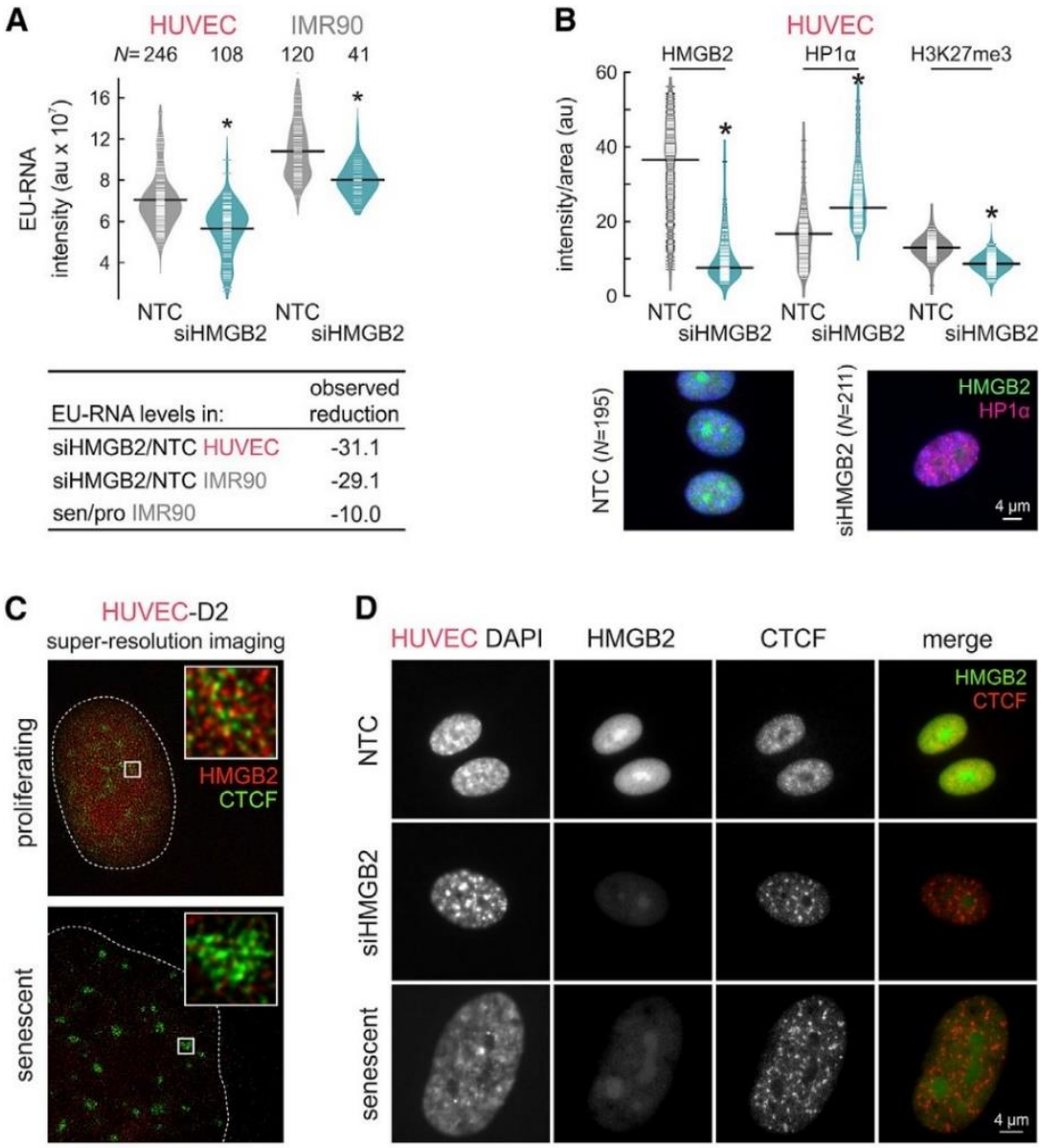

E

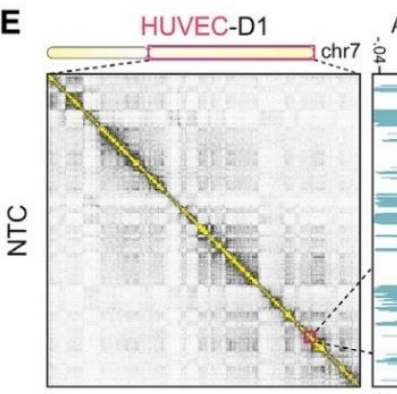

$\begin{array}{r}\mathrm{A} / \mathrm{B} \\ \dot{0} \\ \dot{1} \\ \hline\end{array}$
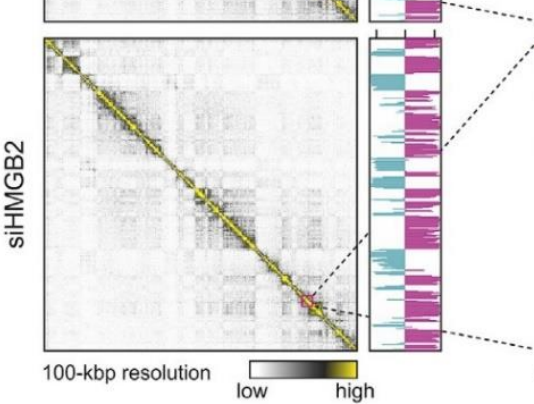

$\mathbf{F}$

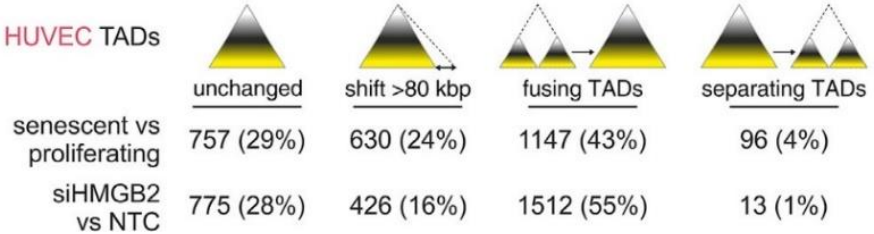

Figure3.1.6. HMGB2 knockdown alters CTCF and chromatin organization.

(A) Decrease in nascent EU-RNA between control (NTC) and knockdown (siHMGB2) HUVEC or IMR90. *significantly different to NTC; P<0.05, Wilcoxon-MannWhitney test. The total number of cells $(\mathrm{N})$ analyzed is indicated. Average EU-RNA levels reduction in knockdown/control is compared (below) to that in senescent/proliferating cells (normalized for the increase in nuclear size).

(B. Concomitant increase in HP1 $\alpha$ and decrease in H3K27me3 levels in HMGB2-knockdown HUVEC. *significantly different to NTC; $\mathrm{P}<0.05$, Wilcoxon-Mann-Whitney test. Typical images of NTC or siHMGB2 HUVEC immunostained for HMGB2/HP1 $\alpha$ (below); the total number of cells $(\mathrm{N})$ analyzed is indicated.

(C) Super-resolution images of proliferating and senescent HUVEC immunostained for HMGB2 and CTCF. Insets: magnified clusters.

(D) Typical widefield images of control, HMGB2-knockdown or senescent HUVEC immunostained for HMGB2 and CTCF.

(E) Hi-C contact frequencies along the long arm of chr7 in control and HMGB2-knockdown HUVEC. 100 kbp-resolution maps are aligned to A/B compartment plots. Zoom-ins are aligned to HMGB2 and CTCF ChIP-seq and changed interactions around HMGB2 peaks are indicated (arrows).

(F) The number (percentages in brackets) of TADs that remain unchanged, shift one boundary, fuse into one larger TAD, or separate into smaller TADs in senescent/proliferating or HMGB2knockdown/ control HUVEC. 
Results

A

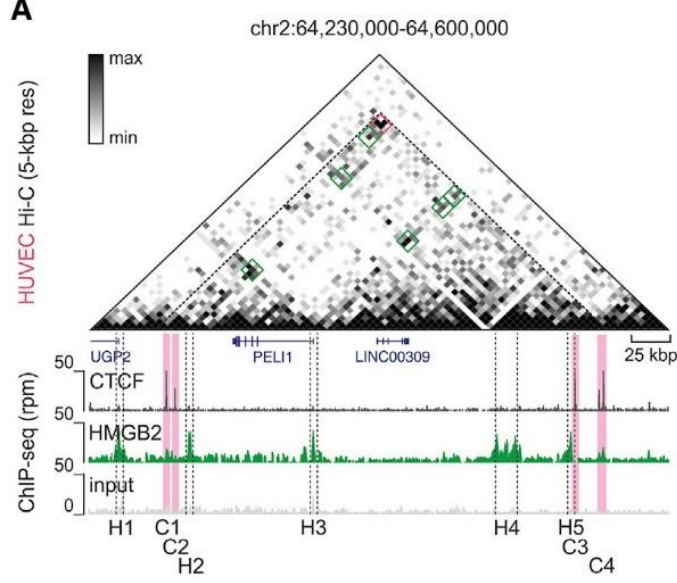

C

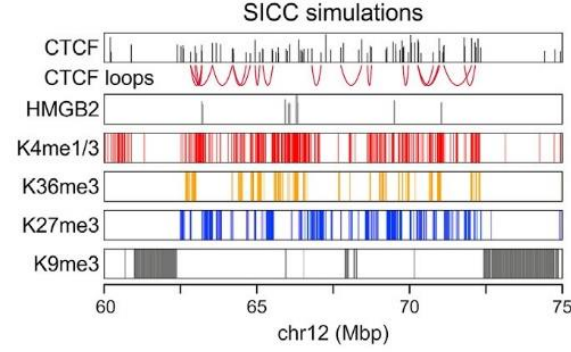

E

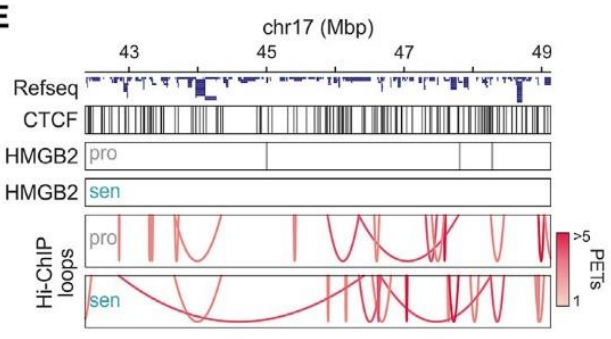

G
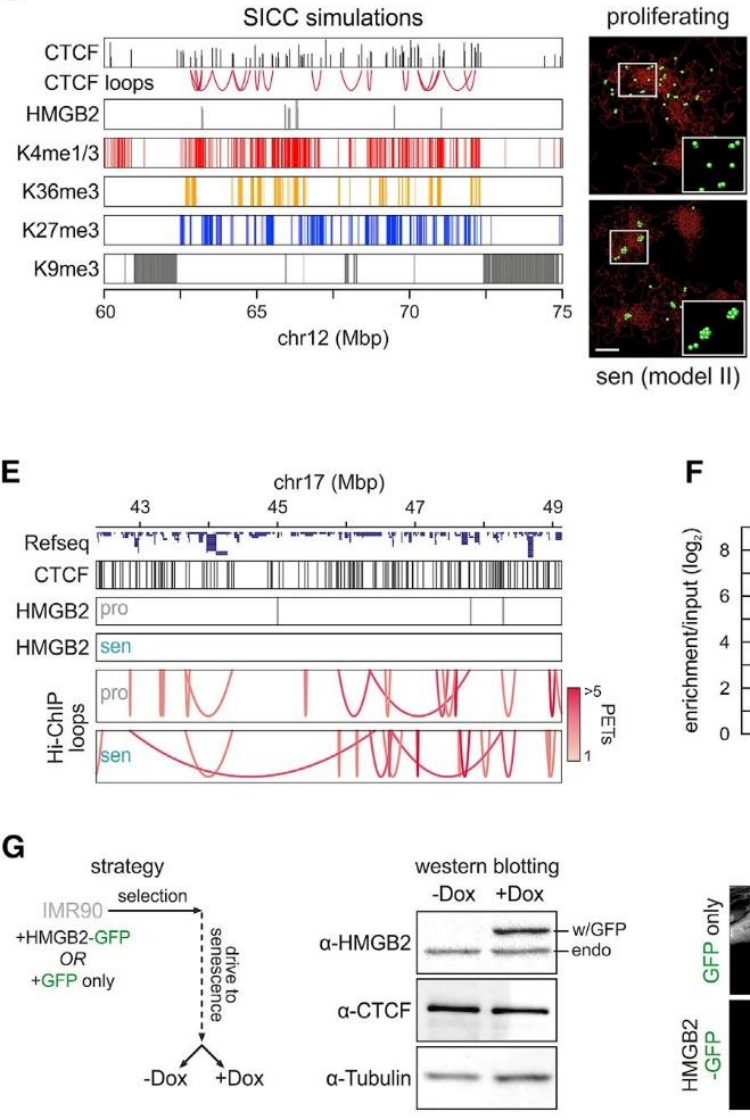
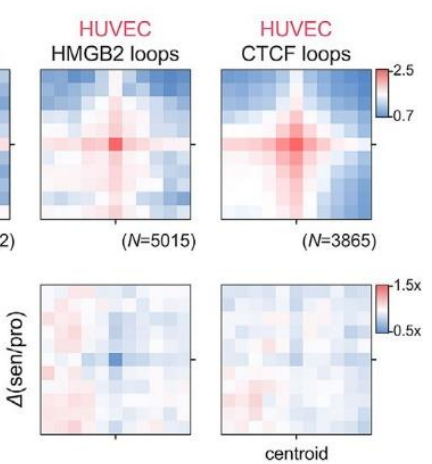

H

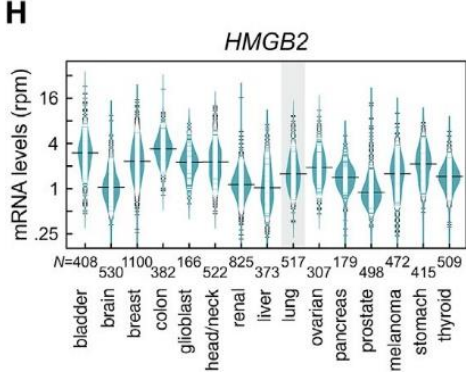

D

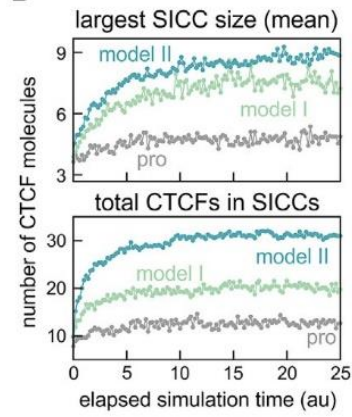

F
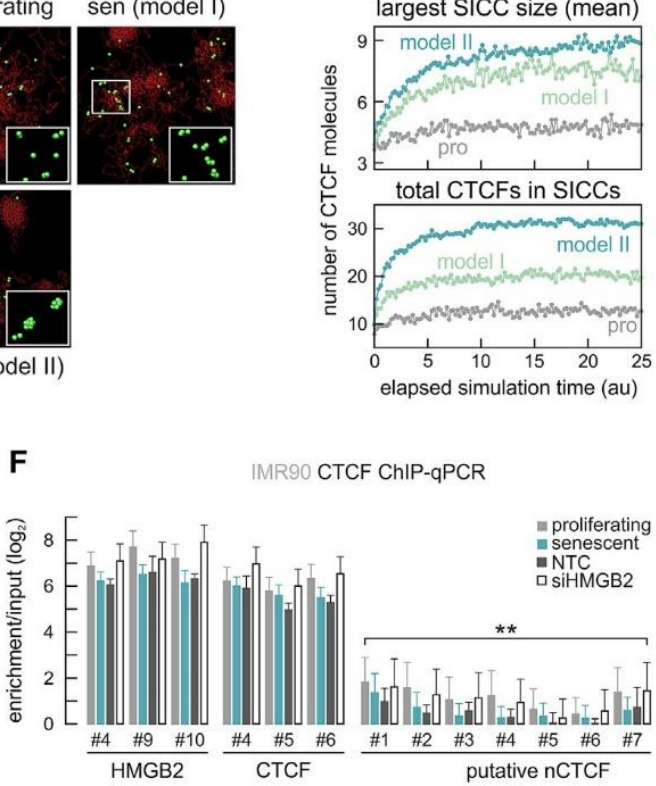
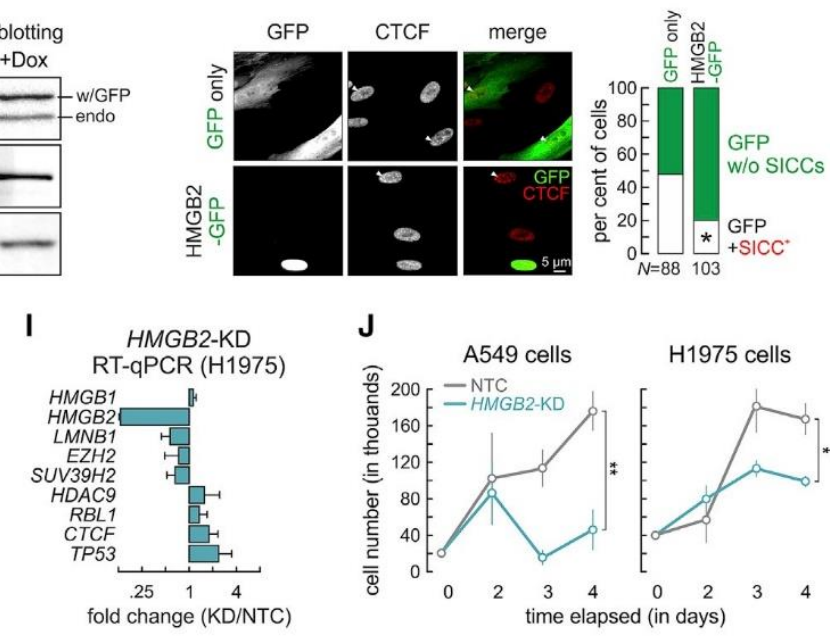

J

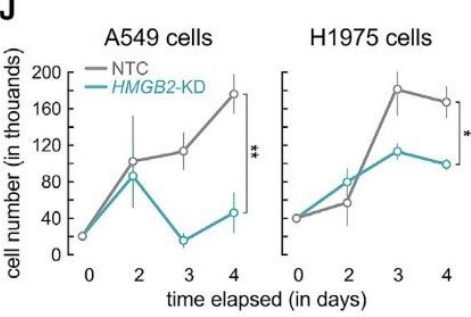


Figure 3.1.7. HMGB2 forms loops and deters CTCF clustering.

(A) CTCF and HMGB2 ChIP-seq from proliferating HUVEC aligned to high-resolution Hi-C (Rao et al., 2014). Loops (local Hi-C signal maxima) involving CTCF (C1-3; magenta) or HMGB2 peaks (H1-5; green) are demarcated.

(B) Average Hi-C signal in the $100 \mathrm{kbp}$ around CTCF-CTCF loops (separated by $\geq 150 \mathrm{kbp}$ ) from proliferating cells (top) or in senescent versus proliferating cells (bottom)..

(C) Left: CTCF peaks and loops, and HMGB2, H3K4me1/3, H3K36me3, H3K27me3, and H3K9me3 ChIP-seq peaks along $15 \mathrm{Mbp}$ of chr12 used in simulations. Right: Snapshots of single conformations from proliferating or senescent-cell models ("I" and "II"). showing chromatin (red) and bound CTCF (green).

(D) Graphs showing increasingly larger SICC sizes (top). and CTCF molecules per SICC (bottom) with simulation time for each of the three models in panel C.

(E) HiChIP CTCF loops in a 6.5-Mbp region of chr17 from proliferating and senescent IMR90. CTCF and HMGB2 peaks in proliferating and senescent IMR90 are aligned above.

(F) Mean ChIP-qPCR enrichment (over input and negative controls; $\log 2 \pm$ S.D., $n=2$ ) in different IMR90 populations with primers targeting CTCF, HMGB2 or putative "neoCTCF" sites.

(G) Left: Strategy for ectopic HMGB2 expression in IMR90. Middle: Overexpression of endogenous ("endo"). or GFP-fusion HMGB2 ("w/GFP"); CTCF and $\beta$-tubulin levels provide a control. Right: Typical images of Doxinduced IMR90 expressing only GFP or HMGB2-GFP (white arrowheads indicate SICCs), and quantification of GFP-positive cells carrying SICCs (white) with the number of cells in each group (N) displayed below. *significantly different; $\mathrm{P}<0.01$, Fisher's exact test.

(H) HMGB2 expression levels across 15 cancer types (http://www.cbioportal.org/); the number of patient samples per each type is indicated, and lung adenocarcinoma data highlighted.

(I) Changes in selected mRNAs levels upon HMGB2 knockdown in H1975 knockdown versus control cells (mean fold change \pm S.D.; $\mathrm{n}=2$ ).

(J) The number of live A549 and H1975 lung adenocarcinoma cells that remain (mean \pm S.D.; $n=3$ ) in the 4 days after HMGB2 knockdown. *significantly different mean; P<0.01, unpaired, two-tailed, Student's t-test.

\subsubsection{Discussion}

Proliferating cells have mechanisms in place that preserve their inherent chromatin state and its higher-order architecture in conjunction with DNA replication and cell division - and 3D chromatin folding gradually dissolves towards mitosis, before being reinstated after cell division concludes (Naumova et al., 2013). However, how this maintenance is achieved in proliferatively-arrested cells remains unclear. Here we hypothesized that entry into senescence of different cell types is governed by a shared regulatory backbone involving an early event of spatial genomic reorganization. We assign this presumed event to the downregulation of genes associated with DNA conformation maintenance, of which HMGB1/B2 stand out due to their nuclear abundance and inherent ability to bend chromatin (Stros, 2010).

At least $10 \%$ of HMGB-bound loci mark TAD boundaries, often coinciding with active gene promoters. This is consistent with active protein-coding and tRNA genes overrepresented at TAD boundaries (Dixon et al., 2012) and the notion that transcription acts to partition eukaryotic genomes (Rowley et al., 2017). At HMGB-marked TAD boundaries, the change in activity of the underlying genes coincides with interaction reshuffling upon senescence entry. Notably, HMGB2 contributes to loop formation along human chromosomes, much like CTCF, and this unveils a novel looping factor specifically contributing to $3 \mathrm{D}$ genome conformation in proliferating primary cells. 
Unlike manipulations of HMGB1 in fibroblasts (Davalos et al., 2013), HMGB2 loss does not suffice for senescence induction given the transient nature of a knockdown experiment. However, its suppression recapitulated three key features of senescence entry: It globally reduced transcriptional output, yielding as much as $30 \%$ less nascent RNA, induced a shift between facultative and constitutive heterochromatin, and HMGB2-bound TAD boundaries collapsed as regards interaction insulation. All three effects were widespread and constitute prime examples of genomic function following form, highlighting how replicative senescence is a suitable and perhaps underappreciated model for studying this relationship in human chromosomes.

Senescent cells are also characterized by the dramatic clustering of CTCF foci into SICCs. In proliferating cells, HMGB2-bound positions seem to insulate flanking CTCF peaks from forming longrange interactions with one another; this is alleviated upon senescence entry. Notably, SICC formation is reminiscent of "phase separation" (Hnisz et al., 2017), but proved reversible upon reintroduction of HMGB2, which should perhaps be expected for an early event on the path to senescence. Another notable feature is that HMGB1/B2 have been detected bound to mitotic chromatin (Pallier et al., 2003) in a manner similar to another HMGB-box factor, Sox2 (Teves et al., 2016). This opens up the possibility of mitotic bookmarking by HMGB2, thus connecting proliferative capacity to topological demarcation and looping. Although further work is needed, such bookmarking could explain the inability of lung adenocarcinoma cells to propagate in the persistent absence of HMGB2. We propose that deregulation of higher-order chromatin organization via the nuclear depletion of HMGB2 constitutes a deterministic primer for the ensuing senescent program across cell types, and HMGB2 itself acts as a rheostat of topological insulation, the depletion of which reversibly affects global transcription competency and heterochromatin organization.

\subsubsection{Materials and Methods}

\section{Primary human cell lines and senescence markers}

HUVECs from single, apparently healthy, donors (passage 2; Lonza Inc.) were continuously passaged to replicative exhaustion in complete Endopan-2 supplemented with $2 \%$ FBS under $5 \% \mathrm{CO}^{7}$. Cells were constantly seeded at $\sim 10,000$ cells $/ \mathrm{cm} 2$, except late passages that were seeded at $\sim 20,000$ cells/cm2. Single IMR90 isolates (I90-10 and -79, passage 5; Coriell Biorepository) were continuously passaged to replicative exhaustion in MEM (M4655, Sigma-Aldrich) supplemented with non-essential amino acids and 20\% FBS under 5\% $\mathrm{CO}_{2}$. Mesenchymal stromal cells (MSCs) were isolated at the Aachen Medical School, and cultured to senescence as previously described (Franzen et al., 2017). Cell proliferation was monitored by MTT assays at different passages. In brief, 5,000 cells are seeded in 96-well format plates in quadruplicates. On the next day, the medium is replaced with $100 \mu \mathrm{l}$ fresh 
medium plus $10 \mu 1$ of a $12 \mu \mathrm{M}$ MTT stock solution (Invitrogen), and cells are incubated at $37^{\circ} \mathrm{C}$ for 4 h. Subsequently, all but $25 \mu \mathrm{l}$ of the medium is removed from the wells, and formazan dissolved in 50 $\mu 1 \mathrm{DMSO}$, mixed thoroughly and incubated at $37^{\circ} \mathrm{C}$ for $10 \mathrm{~min}$. Samples are then mixed again and absorbance read at $530 \mathrm{~nm}$. Measurements are taken at 24, 48 and $72 \mathrm{~h}$ post-seeding, background subtracted, and normalized to the $24 \mathrm{~h}$ time point. Senescence-associated $\beta$-galactosidase assay (Cell Signaling) was performed according to the manufacturer's instructions to evaluate the fraction of senescent cells at different passages. Finally, DNA methylation at six selected $\mathrm{CpG}$ islands (Franzen et al., 2017) was measured by isolating genomic DNA at the different cell states and performing targeted pyrosequencing (Cygenia $\mathrm{GmbH})$.

\section{Immunofluorescence and image analysis}

Cells were grown on coverslips from the stage indicated; they were fixed in 4\% PFA/PBS for 15 min at room temperature. After washing once in PBS, cells were permeabilized with $0.5 \%$ Triton-X/PBS for 5 min at room temperature. Blocking with $1 \%$ BSA/PBS for $1 \mathrm{~h}$ was followed by incubation with the indicated primary antibodies for 1-2 h. Cells were washed twice with PBS for $5 \mathrm{~min}$ before incubating with secondary antibodies for $1 \mathrm{~h}$ at room temperature. Nuclei were stained with DAPI (Sigma-Aldrich) for $5 \mathrm{~min}$, washed, and coverslips mounted onto slides in Prolong Gold Antifade (Invitrogen).. For image acquisition, a widefield Leica DMI 6000B with a HCX PL APO 63x/1.40 (Oil) objective was used; confocal and super-resolution images were acquired on a Leica TCS SP8 gSTED microscope with a 100x/1.40 (Oil) STED Orange objective. Deconvolution of super-resolution images was performed using the Huygens software from Scientific Volume Imaging. Note that for the immunostainings of preextracted nuclei (Figure 3.1. S9F), cells grown on overspills were incubated in CSK buffer (10 mM HEPES pH 7.9, $300 \mathrm{mM}$ sucrose, $100 \mathrm{mM} \mathrm{Nacl}, 3 \mathrm{mM} \mathrm{MgCl}$ ) for $1 \mathrm{~min}$ at room temperature, and then in $\mathrm{CSK}+0.5 \%$ Triton $\mathrm{X}-100$ for $1 \mathrm{~min}$ at room temperature before fixation as described above.

\section{Genome-wide chromosome conformation capture (Hi-C) and analysis}

For Hi-C studies, 25-35 million proliferating/senescent HUVEC were crosslinked by adding 1\% PFA (Electron Microscopy Sciences) for $10 \mathrm{~min}$ at room temperature with subsequent quenching (125 mM glycine), and gently scraped off the plates on ice. Then, Hi-C was performed as previously described (Belton et al., 2012) under conditions where nuclei remained largely intact. In brief, cells were lysed (10 $\mathrm{mM}$ Tris-HCl pH 8.0, $10 \mathrm{mM} \mathrm{NaCl}, 0.4 \% \mathrm{NP} 40,1 \mathrm{x}$ protease inhibitor cocktail) twice for $20 \mathrm{~min}$ on ice, washed with 1x NEBuffer 2 twice, and distributed into 4-5 tubes. Following SDS treatment (0.1\%). for $10 \mathrm{~min}$ at $65^{\circ} \mathrm{C}$ and quenching with Triton-X for $15 \mathrm{~min}$ at $37^{\circ} \mathrm{C}$, chromatin was digested with 600 units of HindIII overnight at $37^{\circ} \mathrm{C}$ under constant agitation. Next day, digestion was extended for another hour with an additional 600 units of HindIII for 1-2 hours. DNA overhangs were filled-in using a biotin-

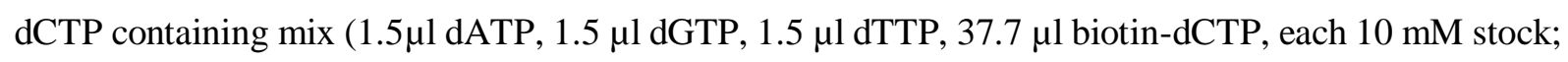
Invitrogen) in the presence of $1.5 \mu 150 \mathrm{U} / \mu 1$ Klenow (NEB) for $45 \mathrm{~min}$ at $37^{\circ} \mathrm{C}$, and all enzymes are inactivated by adding $86 \mu 110 \%$ SDS to all tubes and incubating for $30 \mathrm{~min}$ at $65^{\circ} \mathrm{C}$. Next, each 
individual mixture was transferred into a $15 \mathrm{ml}$ tube, incubated for 10-20min on ice to quench SDS, and a mixture containing $745 \mu \mathrm{l}$ of 10\% Triton-X, $745 \mu \mathrm{l}$ 10x ligation buffer (500 mM Tris-HCl pH 7.5, 100 $\mathrm{mM} \mathrm{MgCl} 2,100 \mathrm{mM}$ DTT), $80 \mu \mathrm{l}$ of a $10 \mathrm{mg} / \mathrm{ml}$ BSA stock, $80 \mu \mathrm{l}$ of $100 \mathrm{mM}$ ATP and $5.96 \mathrm{ml}$ milliQ water is added to each tube, and blunt-end ligation initiated by adding $15 \mu 1$ of 5 U/ $\mu 1$ T4 DNA ligase (Invitrogen) and incubated for 6 hours at $16^{\circ} \mathrm{C}$. Crosslinks were then reversed and proteins degraded by adding $35 \mu \mathrm{l}$ of $15 \mathrm{mg} / \mathrm{ml}$ proteinase $\mathrm{K}$ and incubated overnight at $65^{\circ} \mathrm{C}$. Next day, an additional $35 \mu \mathrm{l}$ of proteinase $\mathrm{K}$ was added for 2 hours at $65^{\circ} \mathrm{C}$, mixtures were cooled to room temperature, transferred to 50-ml tubes, and DNA was extracted twice with phenol:chloroform $(\mathrm{pH} \mathrm{8.0)}$ and ethanol precipitated. The resulting pellets were dissolved in $450 \mu \mathrm{l} 1 \mathrm{x}$ TE, transferred to $1.7 \mathrm{ml}$ tubes, DNA was re-extracted twice, precipitated, pellets were washed once in 70\% ethanol, air-dried, and resuspended in $25 \mu 1$ x TE. Subsequently, three tubes, each with $\sim 5 \mu \mathrm{g}$ of the Hi-C template, were incubated for $2 \mathrm{~h}$ at $12^{\circ} \mathrm{C}$ in the presence of $1 \mu 110 \mathrm{mg} / \mathrm{ml}$ BSA, $10 \mu \mathrm{l}$ 10x NEBuffer 2, $1 \mu 110 \mathrm{mM}$ dATP, $1 \mu 110 \mathrm{mM}$ dGTP (for NcoI samples dCTP was used instead) and 5 units of T4 DNA polymerase (NEB). in a total volume of $100 \mu 1$ to remove biotin-16-dCTP at non-ligated DNA ends. Reactions were stopped by adding $2 \mu 1$ of $0.5 \mathrm{M}$ EDTA pH 8.0, and the DNA was subsequently purified by phenol extraction and ethanol precipitation. DNA pellets were resuspended and pooled in a total of $100 \mu 1$ of milliQ water, and residual salts were removed via Amicon 30K columns via three washing steps (1x $200 \mu 1$ and 2x $100 \mu 1$ milliQ water). Finally, DNA was sheared to a size of 300-600 bp on a Bioruptor Plus (Diagenode; 2x 10 cycles of 30 sec on and $30 \mathrm{sec}$ off, at the highest power setting).

For IMR90 and MSC cells, the procedure was slightly modified to follow the in situ Hi-C protocol (Rao et al., 2014).. Approx. 20 million cells were lysed twice in $900 \mu 1$ lysis buffer (10 mM Tris-HCl pH 8.0, $10 \mathrm{mM} \mathrm{NaCl}, 0.4 \% \mathrm{NP}-40,1 \mathrm{x}$ protease inhibitor cocktail) for $15 \mathrm{~min}$ on ice, nuclei were pelleted and washed once in 1x NEBuffer 2, distributed into 4 tubes, resuspended in $200 \mu 10.5 \%$ SDS/NEBuffer 2, and incubated for $10 \mathrm{~min}$ at $62^{\circ} \mathrm{C}$. After repelleting, SDS-treated nuclei were resuspended in $150 \mu 11 \%$ Triton-X/NEBuffer 2, and digested overnight using 400 units of HindIII at $37^{\circ} \mathrm{C}$ with constant agitation. Next day, another 400 units of HindIII were added for another $1-2 \mathrm{~h}$ at $37^{\circ} \mathrm{C}$. Following heat inactivation for $20 \mathrm{~min}$ at $62^{\circ} \mathrm{C}$, nuclei were pelleted by centrifugation and resuspend in $256 \mu 1$ NEBuffer 2. DNA ends were then biotinylated by adding $50 \mu 1$ of a biotin-dCTPKlenow mix (37.5 $\mu \mathrm{l}$ of $0.4 \mathrm{mM}$ biotin-dCTP, $1.5 \mu \mathrm{l}$ of $10 \mathrm{mM}$ dTTP, dATP, and dGTP, and $1.5 \mu \mathrm{l}$ of 50 $\mathrm{U} / \mu \mathrm{l}$ Klenow). to each tube and incubating for $1 \mathrm{~h}$ at $37^{\circ} \mathrm{C}$. Subsequently, $900 \mu \mathrm{l}$ of ligation mix (603 $\mu 1 \mathrm{H} 2 \mathrm{O}, 240 \mu 15 \mathrm{x}$ Invitrogen ligation buffer, $30 \mu 1$ of $10 \%$ Triton-X, $12 \mu \mathrm{l}$ of $10 \mathrm{mg} / \mathrm{ml} \mathrm{BSA}$, and 15 $\mu 1$ ligase) was added to each sample, mixed, and incubated for $6 \mathrm{~h}$ at $16^{\circ} \mathrm{C}$; tubes were inverted frequently. Nuclei were next pelleted and a volume of $900 \mu \mathrm{l}$ was removed. Following reversal of crosslinking and proteinase $\mathrm{K}$ addition, DNA is phenol:chloroform extracted and ethanol precipitated; all subsequent steps were performed as described above. 
Hi-C DNA was used as template for adding sequencing linkers via 10 PCR cycles. Then, Hi-C libraries were sequenced to generate at least 400 million paired-end reads (50-75 bp in length) per each donor/condition on a HiSeq4000 platform (Illumina). Raw reads were mapped to the human reference genome (hg19) iteratively (to ensure maximum recovery of uniquely mapped pairs). using BWA (Li \& Durbin, 2010), duplicates were removed (http://picard.sourceforge.net/), and the output converted into BEDPE format. Next, custom R scripts were used to bin the genome into non-overlapping bins (typically 10-kbp ones), assign reads to bins, remove read pairs not representing valid interactions, and to normalize read counts to library size. Finally, the HiTC Bioconductor package was used to annotate, correct matrices for biases in genomic features (Servant et al., 2012), and visualize 2D heat maps at different resolutions. In addition, we applied a composite, uniform, correction to Hi-C maps from senescent HUVEC due to their tetraploidy; the scaling factor was calculated by adjusting for the ratio of $2 \mathrm{n}$ versus $4 \mathrm{n}$ cells in the two HUVEC populations (as recorded using DNA FISH; Figure 3.1. S6A), as well as the shift in cis-/trans-contact ratio (expecting the tetraploid cells will have a small bias in favour of cis-interactions. The final factors used were $0.961 \mathrm{x}$ for D1, and $0.641 \mathrm{x}$ for $\mathrm{D} 2$, and were applied before data merging. For subtracted Hi-C maps, we determined bins under a particular cutoff (typically $5 \mathrm{rpm}$ ) and set them to 0 , while all others were set to 1 ; then, matrices were subtracted to give a binary appreciation of the interaction changes between the different states. For plotting insulation heatmaps, normalized interactions values in the twenty 20-kbp bins around each HMGB1/B2 peak were added up, normalized to the median value in each bin and plotted. All R scripts used here are available on request, and all HMGB2-HMGB2 loops identified are listed in Table S1. Note that the visualization of high-resolution Hi-C data (from Rao et al., 2014) was via the "3D Genome Browser" interface (http://promoter.bx.psu.edu/hi-c/).

\section{RNA isolation, sequencing, and analysis}

Cell of different types/conditions were harvested in Trizol LS (Life Technologies) and total RNA was isolated and DNase I-treated using the Direct-zol RNA miniprep kit (Zymo Research). Following, rRNA-depletion using the RiboZero Gold kit (Illumina), barcoded cDNA libraries were generated using the TruSeq RNA library kit (Illumina) with (IMR90, MSC) or without selection on poly(dT) beads (HUVEC); in addition some IMR90 and MSC libraries were spiked using a synthetic ERCC Spike-In mix (Thermo Fischer Scientific) to facilitate normalization. The resulting libraries were paired-end sequenced to at least 50 million read pairs on a HiSeq4000 platform (Illumina)., and raw reads were mapped to the human reference genome (hg19) and analyzed via the QuickNGS pipeline (Wagle et al., 2015) to obtain read counts. Then, read counts from different single-donors/conditions were normalized using the RUVSeq package (Risso et al., 2014). Please note that, in our hands, normalization using either synthetic spike-in controls or the intrinsic "top quantile" function of the RUVseq package is essentially interchangeable. Finally, for analysis of nascent RNA in IMR90 the "factory RNA-seq" approach was applied on $\sim 5$ million proliferating or senescent cells (Melnik et al., 2016), RNA was isolated and 
sequenced as above, and intronic read counts were obtained and differentially analyzed for the two conditions using the iRNAseq package (Madsen et al., 2015). All box/bean plots were plotted using the BoxPlotR tool online (http://shiny.chemgrid.org/boxplotr/), and GO term/pathway enrichment analyses using Metascape (http://metascape.org/gp/index.html; Tripathi et al., 2015). Differentially-regulated genes per each cell type are listed in Table $\mathrm{S} 2$.

\section{D-DNA FISH and high-throughput imaging}

A three color DNA FISH was performed using the BAC probes RP11-347B21, RP11-296G9 and RP11$781 \mathrm{M} 19$ (Chori BACPAC) fluorescently labeled with Alexa-488 dUTP, Alexa 568 dUTP and Alexa 647 dUTP, respectively by nick translation. Briefly, HUVEC and IMR90 cells grown and fixed on coverslips were permeabilized in $0.5 \%$ saponin $/ 0.5 \%$ tritonX100/PBS for 20 minutes and incubated in $0.1 \mathrm{~N} \mathrm{HCl}$ for 15 minutes. Following a 30-min incubation in 50\% formamide/2X SSC, samples were denatured with the labelled BACs at $85^{\circ} \mathrm{C}$ for $5 \mathrm{~min}$, and hybridized at $37^{\circ} \mathrm{C}$ overnight. Three washing steps with 1xSSC and 0.1x SSC were performed before mounting of coverslips with Vectashield containing DAPI onto glass slides. Images were acquired with an Opera Phenix High Content Screening System (PerkinElmer)., equipped with four laser lines (405 nm, $488 \mathrm{~nm}, 568 \mathrm{~nm}$, and $640 \mathrm{~nm}$ ) and two 16-bit CMOS cameras. Images for 3D and radial distances were acquired in confocal mode using a 40X water objective lens (NA 1.1). For each sample, 13 z-planes separated by $0.5 \mu$ m were obtained for a total number of at least 108 fields, on a minimum of 1500 cells per condition. For cell cycle staging by DAPI content analysis, cells were imaged using a 20X water objective lens (NA 1.0) in wide-field mode. For each sample, 3 z-planes separated by $1 \mu \mathrm{m}$ were obtained for a total number of 72 fields, on a minimum of 3500 cells per condition.

\section{Molecular dynamics simulations}

Polymer simulations in Figure 3.1. S6 were performed according to a predictive scheme developed previously (Brackley et al., 2016a; Brackley et al., 2016b). First, the target region (chr12: 60,000,00075,000,000; hg19) was divided into 3-kbp windows, each represented by a polymer "bead". Protein complexes are also represented by simple spheres, which interact attractively with specific beads on the polymer. These are determined using IMR90 ChIP-seq data for H3K4me3, H3K36me3, H3K27me3, and H3K9me3 (ENCODE, 2012). We accordingly specified three types of changing bridging proteins and polymer beads. The first type of protein, denoted "active", interacts with polymer beads marked by H3K4me1/3 and H3K36me3 (binding strongly to the enhancer-/promoter-associated marks, and weakly to the H3K36me3 regions); the second type, denoted "repressed", interacts with beads marked by H3K27me3; the third type, denoted "heterochromatin", interacts with beads marked by H3K9me3. Since polymer beads cover 3-kbp, it was possible for a bead to cover regions in different states, and so interact with more than one type of protein; beads not carrying any mark do not interact attractively with any bridging proteins. The dynamics of the diffusing polymer and of the protein complexes are simulated using LAMMPS (Plimpton, 1995) in "Langevin Dynamics" mode; the position of each bead is 
determined by an equation that describes the potential for interactions between all elements in the system. These potentials include: spring bonds between adjacent beads along the polymer (finite extensible non-linear elastic bonds), angle interactions between triplets of adjacent polymer beads (giving the polymer bending rigidity), steric interactions between all beads to prevent them from overlapping (Weeks-Chandler-Anderson potential), and attractive interactions between protein spheres and polymer beads (shifted truncated Lennard-Jones potential). We performed 48 independent simulations of this 5000-bead polymer with 167 active, 109 H3K27me3-binding, and 165 H3K9me3binding proteins (these protein numbers were chosen such that the ratio of binding sites to proteins is as previously found to give an optimal prediction of Hi-C data; Brackley et al., 2016b). The system extends 120 -bead diameters $(\sigma)$, the persistence length of the polymer is $4-\sigma$, and we use interaction energies of $5.5 \mathrm{kBT}$ and $2 \mathrm{kBT}$ for the strong and weak Lennard-Jones interactions, respectively (with a cut-off of $1.8-\sigma)$. Each 3-kbp polymer bead has a 30-nm diameter, and each simulation is run for the equivalent of $76 \mathrm{sec}$. These simulations modelled proliferating cells; to model senescent cells a further set of 48 simulations were performed where we increased the number of $\mathrm{H} 3 \mathrm{~K} 9 \mathrm{me} 3$-binding proteins by $30 \%$ to 220.

For the CTCF clustering simulations in Figure 3.1.7, in addition to protein bridges, we also included additional spring interactions between pairs of CTCF sites such that permanent loops form. We used HUVEC ChIP-seq data for H3K4me3, H3K36me3, H3K27me3, and H3K9me3 (ENCODE, 2012) as above to label beads as binding for "active", "repressed" or "heterochromatin" proteins. In each of 48 independent simulations, pairs of convergent CTCF sites are stochastically chosen to form loops depending on the ChIP-seq signal strength in HUVEC data - in this way, a different set of convergent CTCF sites could be looped in each simulation, allowing for cell-to-cell variation. Operating on the hypothesis that CTCF looping is disrupted by the high mobility group proteins, in the case of proliferating cells we switched off any CTCF loops which enclosed HMBG2 binding sites or for which an HMGB2 sites was adjacent to the CTCF site; additionally we switched off protein-bridge binding to beads adjacent to all HMGB2 and CTCF sites. As before the number of heterochromatin binding bridges was $30 \%$ higher in the senescent cell simulations. Another possible mechanism for CTCF clustering in senescence is that in these cells there are multimeric interactions between CTCFs (i.e., more than pairwise binding, either directly between CTCF proteins or mediated by another protein); to model this scenario we perform a set of simulations as above, but which also include a direct attractive interaction between all CTCF sites (i.e., fixed-spring interactions between convergent pairs, plus short range attraction between all CTCFs; models "I" and "II" in Figure 3.1. 7). 


\section{ChIP-seq and analysis}

For each batch of ChIP experiments approx. 25 million cells, cultured to $>80 \%$ confluence in $15-\mathrm{cm}$ dishes, were crosslinked in $15 \mathrm{mM}$ EGS/PBS (ethylene glycol bis(succinimidyl succinate); Thermo) for $20 \mathrm{~min}$ at room temperature, followed by fixation for $40 \mathrm{~min}$ at $4^{\circ} \mathrm{C}$ in $1 \%$ PFA. From this point onwards, cells were processed via the ChIP-IT High Sensitivity kit (Active motif) as per manufacturer's instructions. Chromatin was sheared to 200-500 bp fragments on a Bioruptor Plus (Diagenode; 2x 9 cycles of $30 \mathrm{sec}$ on and $30 \mathrm{sec}$ off, at the highest power setting), and immuno-precipitation was carried out by adding $4 \mu \mathrm{g}$ of the appropriate antiserum (HMGB1: PCRP-HMGB1-4F10-s, DSHB; HMGB2: ab 67282, Abcam; CTCF: 61311, Active motif) to approx. $30 \mu \mathrm{g}$ of chromatin and incubating on a rotator overnight at $4^{\circ} \mathrm{C}$ in the presence of protease inhibitors. Following addition of protein $\mathrm{A} / \mathrm{G}$ agarose beads and washing, DNA was purified using the ChIP DNA Clean \& Concentrator kit (Zymo Research) and used in qPCR or next-generation sequencing on a HiSeq4000 platform (Illumina). Where ChIP-seq was performed, at least 35 million reads were obtained and the respective "input" sample was also sequenced. Raw sequencing reads (typically 100 bp-long) were mapped to the reference human genome (hg19). using BWA (Li \& Durbin, 2010), and the resulting .BAM files were processed via the MACS2 software (Zhang et al., 2008) to identify signal enrichment over input. Thresholded HMGB1/B2 ChIPseq peaks per each cell type are listed in Table S1, and oligonucleotides used as primers in ChIP-qPCR listed in Table 3.1.S4. The .BAM files were also used in ngs.plot (Shen et al., 2014) for plotting ChIPseq coverage over particular genomic positions for different conditions/cell types. Finally, transcription factor recognition motif enrichments within DHS footprints under HMGB1/B2 ChIP-seq peaks were calculated using the Regulatory Genomics Toolbox (Gusmao et al., 2014).. Note that for the H3K27ac/me3 ChIP-seq, crosslinking was with $1 \%$ PFA for $10 \mathrm{~min}$ at room temperature, rabbit polyclonal antisera were used (H3K27me3: C15410069, H3K27ac: C15410174; Diagenode), and preparations were spiked with chromatin prepared from Drosophila S2 cells as described (Orlando et al., 2014).

\section{CTCF-HiChIP and analysis}

For HiChIP (Mumbach et al., 2016) 30 million proliferating or senescent IMR90 and HUVEC were crosslinked in 1\% PFA for $10 \mathrm{~min}$ at room temperature with subsequent quenching (125 mM glycine), and gently scraped off the plates on ice. Then, the in situ Hi-C protocol described above was applied until the ligation step, and followed by CTCF-bound chromatin immunoprecipitation. Briefly, cells were lysed twice (10 mM Tris- $\mathrm{HCl} \mathrm{pH} 8.0,10 \mathrm{mM} \mathrm{NaCl}, 0.4 \%$ NP-40, 1x protease inhibitor cocktail) for 15 min on ice, nuclei were pelleted and washed once in 1x NEBuffer 2, distributed into 3 tubes, treated with SDS, and incubated for $10 \mathrm{~min}$ at $62^{\circ} \mathrm{C}$. Chromatin was digested using HindIII (for IMR90). or $\mathrm{NcoI}$ (for HUVEC) at $37^{\circ} \mathrm{C}$. Following heat inactivation, DNA ends were biotinylated by a biotin-dCTPKlenow mix and incubated for $1 \mathrm{~h}$ at $37^{\circ} \mathrm{C}$. Subsequently, ligation was performed for $6 \mathrm{~h}$ at $16^{\circ} \mathrm{C}$, during which tubes were frequently inverted. Nuclei were next pelleted, and $260 \mu \mathrm{l}$ of shearing buffer $(0,1 \%$ 
SDS, $1 \mathrm{mM}$ EDTA, $10 \mathrm{mM}$ Tris- $\mathrm{HCl} \mathrm{pH}$ 8.0) was used to resuspend the pellet. Sonication was performed using a Bioruptor (Diagenode; 2x 6 cycles of $30 \mathrm{sec}$ on/30 sec off, at a low power setting). Immunoprecipitation was carried out by adding $4 \mu \mathrm{g}$ of the appropriate antiserum ( $\alpha$-CTCF; 61311, Active motif) to approximately $30 \mu \mathrm{g}$ of biotinylated chromatin before incubating on a rotating platform overnight at $4^{\circ} \mathrm{C}$, in the presence of protease inhibitors. Following addition of protein-G magnetic beads (Active motif)., DNA was purified using the Chip DNA Clean concentrator kit (Zymo Research). HiChIP DNA was used as template for adding Illumina sequencing linkers via 12 PCR cycles, and libraries were sequenced to generate $>150$ million paired-end reads ( $75-100 \mathrm{bp}$ in length) per condition on a HiSeq4000 platform (Illumina). Raw reads were processed and loops identified using "hichipper" (Lareau et al., 2018).

\section{Protein extraction and western blotting}

For assessing protein abundance at the different cell states, approx. $4 \times 106$ cells were gently scraped off 15-cm dishes, and pelleted for $5 \mathrm{~min}$ at $600 \mathrm{x} \mathrm{g}$. The supernatant was discarded, and the pellet resuspended in $100 \mu \mathrm{l}$ of ice-cold RIPA lysis buffer (20 mM Tris-HCl pH 7.5, $150 \mathrm{mM} \mathrm{NaCl}, 1 \mathrm{mM}$ EDTA pH 8.0, 1 mM EGTA pH 8.0, 1\% NP-40, 1\% sodium deoxycholate) containing 1x protease inhibitor cocktail (Roche)., incubated for $20 \mathrm{~min}$ on ice, and centrifuged for $15 \mathrm{~min}$ at $>20,000 \mathrm{x} \mathrm{g}$ to pellet cell debris and collect the supernatant. The concentration of the nuclear extracts was determined using the Pierce BCA Protein Assay Kit (Thermo Fisher Scientific), before extracts were aliquoted and stored at $-70^{\circ} \mathrm{C}$ to be used for western blotting. Resolved proteins were detected using the antisera and dilutions listed in the Key Resources Table.

\section{EdU and EU labeling of nucleic acids}

Nascent DNA synthesis was monitored by EdU incorporation and subsequent labelling with the ClickiT® chemistry (Click-iT EdU Imaging Kit; Invitrogen). In brief, cells were incubated in $10 \mu \mathrm{M}$ EdU for $7 \mathrm{~h}$, fixed using 3.7\% PFA/PBS for $15 \mathrm{~min}$ at room temperature, permeabilized, and labeled as per manufacturer's instructions. Similarly, nascent RNA synthesis was monitored by incorporating EU in transcripts by incubating cells in $1 \mathrm{mM}$ of 5 -ethynyl uridine for exactly $5 \mathrm{~min}$ at $37^{\circ} \mathrm{C}$. Cells were then fixed, permeabilized, and labeled via Click-iT chemistry as above. Before imaging on a widefield Leica microscope as described above, cells were also immunostained for HMGB2, stained with DAPI, and mounted onto glass slides.

\section{Single-cell mRNA sequencing and analysis}

Freshly-frozen early- (p. 4) and late-passage HUVEC (p. 16) were thawed, washed once in warm PBS, and subjected immediately to encapsulation in oil droplets. Each droplet contained beads carrying barcoded oligos for cDNA synthesis that allow both cell barcoding and unique transcript identification on a Chromium 10X Genomics platform as per manufacturer's instructions. This way 8,323 early-and 5,269 late-passage cells were processed (with a $0.8 \%$ chance of capturing a cell duplet). Samples from 
each condition were pooled and sequenced separately in two HiSeq2500 lanes each (Illumina) yielding $\sim 30,000$ reads per early- and $\sim 47,000$ reads per late-passage cell. This accounts for a $>50 \%$ sequencing saturation, and returned $>2,500$ and $>3,000$ robustly captured transcripts for early- and late-passage cells, respectively. Raw sequencing data were processed and visualized using default settings in the Cell Ranger suite provided by the manufacturer.

\section{siRNA-mediated HMGB2 knock-down}

HUVEC or IMR90 were seeded at 20,000 cells/cm2 the day before transfection. An Accell-siRNA pool (Dharmacon) against HMGB2, plus a non-targeting control (NTC; fluorescencently-tagged to allow transfection efficiency to be monitored for each experiment), were added to the cells at a final concentration of $1 \mu \mathrm{M}$. Knock-down efficiency and its downstream effects were evaluated at $72 \mathrm{~h}$ after transfection using RT-qPCR and/or immunofluorescence assays. For HUVEC Hi-C, cells were seeded the same way but in $2 \times 15 \mathrm{~cm}$ plates per condition. The cells were crosslinked at $72 \mathrm{~h}$ post transfection and processed as described for IMR90 and MSC Hi-C. Due to the lower cell number ( $\sim 5$ million cells) permeabilized nuclei were kept in a single tube for downstream processes.

\section{SICC rescue experiments}

Doxicycline-inducible overexpression IMR90 cells were generated using PiggyBac transposition. The HMGB2 ORF was TOPO-cloned from cDNA (Invitrogen). Following validation by Sanger sequencing, it was subcloned into the DOX-inducible KA0717 expression vector to generate an HMGB2-GFP fusion. The construct was co-transfected into IMR90 together with transactivator and transposaseencoding vectors (KA0637 and SBI Biosciences \#PB200PA-1, respectively) at a DNA mass ratio of 10:1:3 using Fugene HD (PRomega) as per manufacturer's instructions. Stable, transgene-positive, proliferating IMR90 were selected using $250 \mu \mathrm{g} / \mathrm{ml} \mathrm{G418} \mathrm{(Sigma} \mathrm{Aldrich),} \mathrm{and} \mathrm{driven} \mathrm{into} \mathrm{replicative}$ senescence. HMGB2-GFP expression was induced using doxycycline for $>6 \mathrm{~h}$, and IMR 90 carrying the empty GFP vector served as a control (for more details see Rao et al., 2016).

\section{Quantification and statistical analyses}

\section{Image analyses}

For immunofluorescence image analysis, the NuclearParticleDetector2D of the "MiToBo" software (from version 1.4.3; available at (http://www2.informatik.uni-halle.de/agprbio/mitobo/). was used. Measurements of nuclear immunofluorescence signal were automatically generated using a mask drawn on DAPI staining to define nuclear bounds. Background subtractions were then used to precisely determine the mean intensity per area of each immunodetected protein. All primary antibodies used here are listed in the Key Resources Table. For STED microscopy only, the 2C Pack STED 775 IR-R(1:2000; 2-0032-052-6, Abberior) secondary antibodies were used. 
For 3D-DNA FISH, image analysis was performed using the Harmony software (version 4.4, PerkinElmer) and custom-made scripts. Segmentation of nuclei was performed via algorithm B in maximum-projection images using the DAPI channel. Any cell in the periphery of the image was excluded from further analysis. In each individual nucleus, FISH spots were identified in all three channels using the spot detection algorithm C. Distances between all different spots of all different channels were calculated in 2D using custom-made building blocks, while the z-plane having the maximum pixel intensity of each spot was identified. Single cell information was then exported as text files and custom R scripts were used to calculate Euclidean distances between the different spots in 3D. In order to only select intrachromosomal distances between the three probes, the minimum distance of each spot (each allele) to other fluorescently labelled alleles was identified and plotted. For data analysis, only cells with equal spot number in all three channels were selected, and at least 1000 cells per condition were analyzed. Distances between spot pairs were plotted as box plots with 5-95\% whiskers in GraphPad and significances determined by a Mann-Whitney U test. For determination of radial distances of each spot to the nuclear center and border, custom scripts were built based on previous analyses (Shachar et al., 2015). DNA content analysis was performed and plotted as previously described (Roukos et al., 2015).

\section{Statistical tests}

P-values associated with Student's t-tests and Fischer's exact tests were calculated using GraphPad (http://graphpad.com/)., those associated with the Wilcoxon-Mann-Whitney test using the EDISONWMW tool (Marx et al., 2016), while Pearson's correlation coefficients ( $\rho$ ) using the function embedded in MS Excel (Microsoft). Unless otherwise stated, P-values $<0.01$ were deemed significant. 


\subsubsection{Supplementary Figures}

A

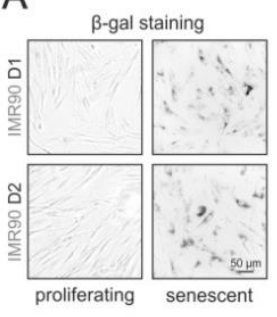

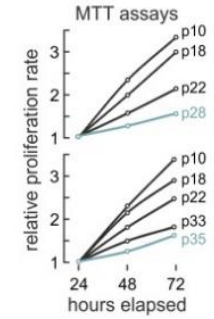

C HUVEC
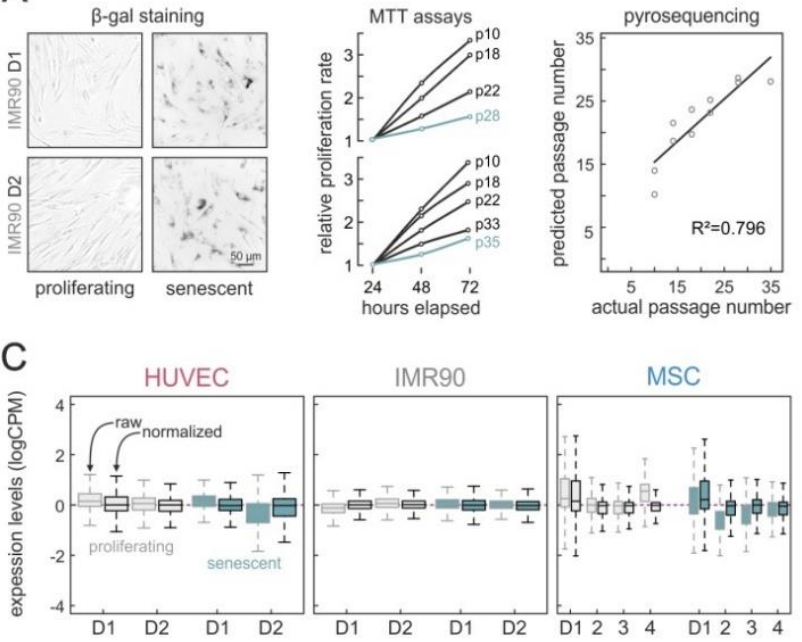

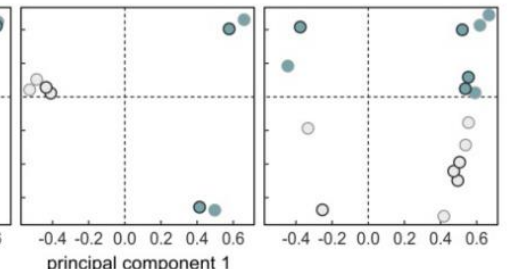
principal component 1

B
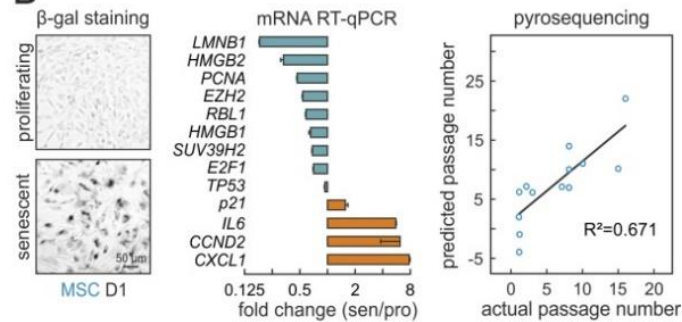

D

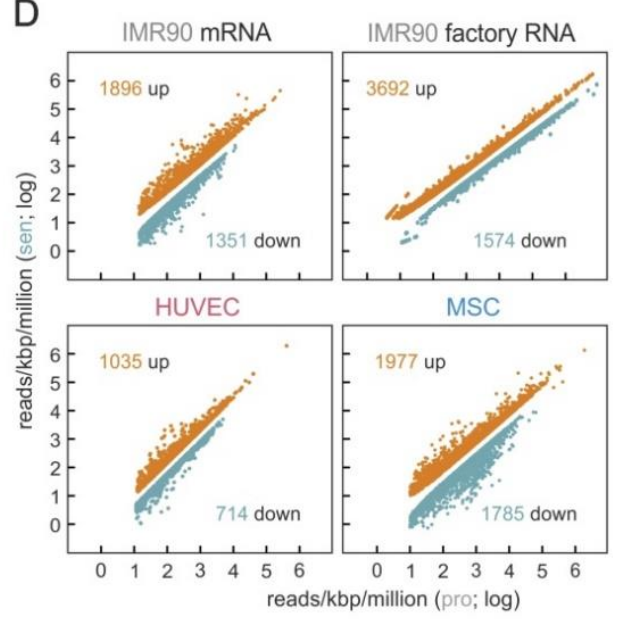

E

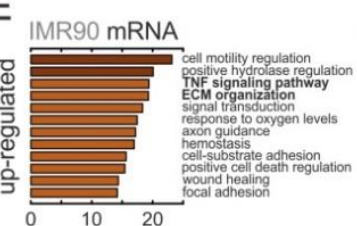

IMR90 factory RNA
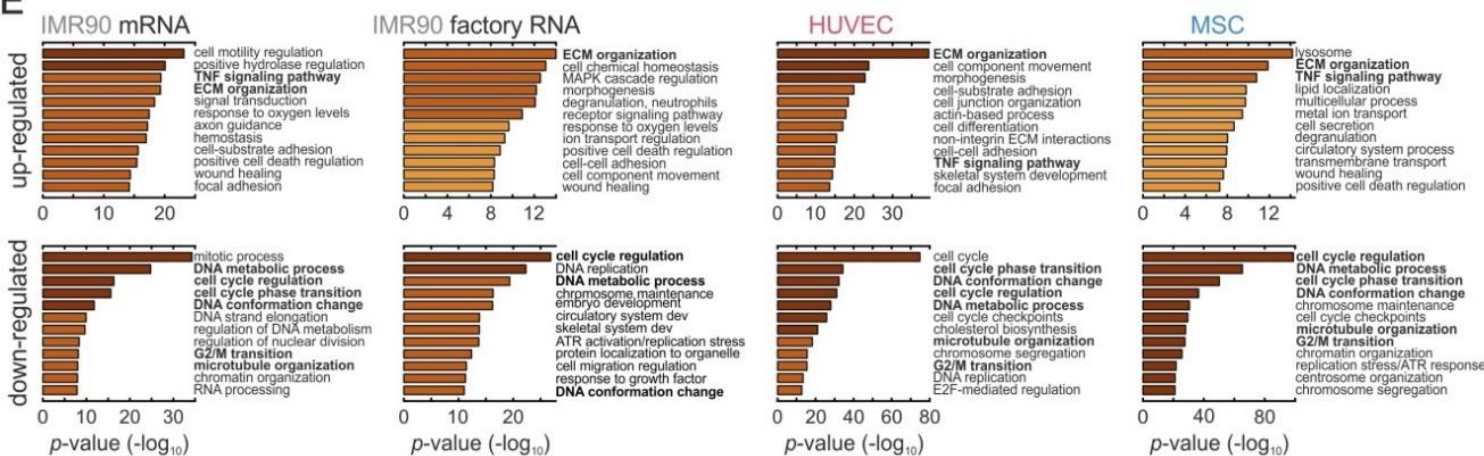

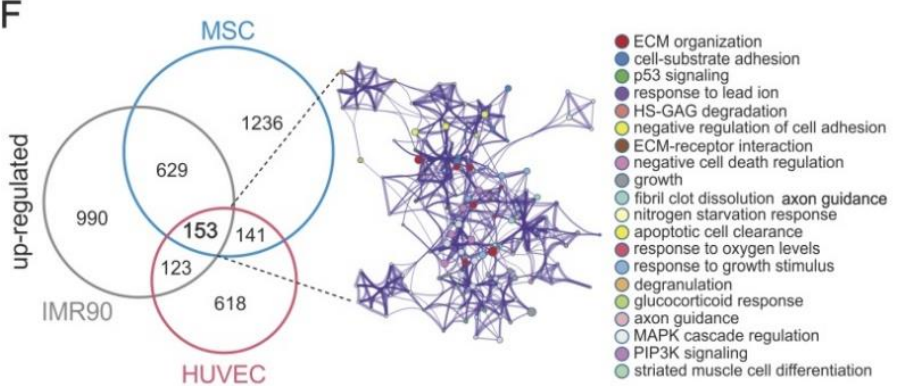

H HUVEC (RT-qPCR)
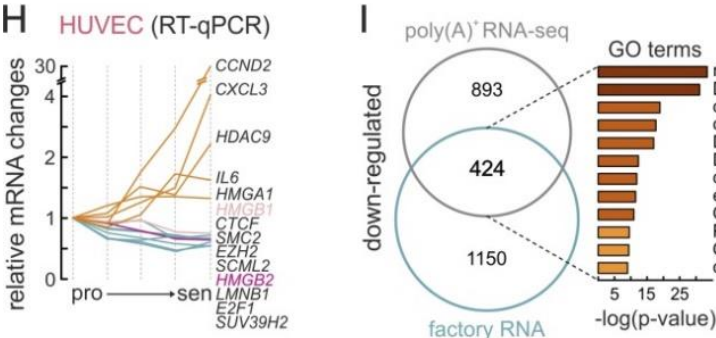

G

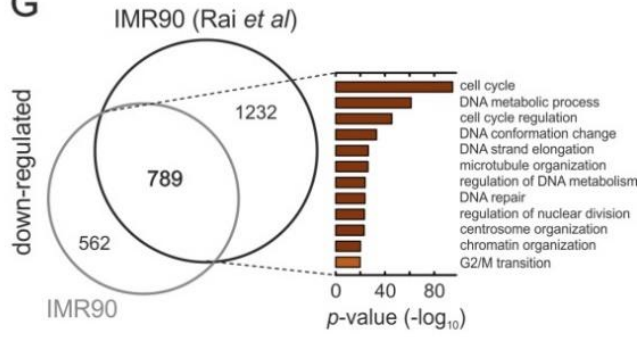

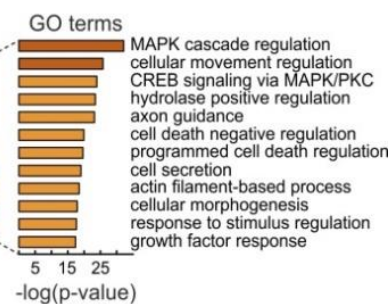


Figure 3.1.S1. Senescence entry determination and RNA-seq data analysis (related to Figure 3.1.1).

(A) Left: $\beta$-galactosidase staining of proliferating and senescent IMR90 from two individual isolates (D1 and D2). Middle: MTT proliferation assays over 72 hours for single-donor IMR90 at different passages; D1 cells in passage 28 , and D2 cells in passage 35 were deemed as senescent (green curves). Right: Plot showing the correlation $\left(\mathrm{R}^{2}\right)$ between actual and predicted passage number based on the methylation levels of six CpGs measured by pyrosequencing.

(B) Left: $\beta$-galactosidase staining of proliferating and senescent MSC from a single donor (D1). Middle: changes in mRNA levels of selected genes were assessed by RT-qPCR. Genes down- (green) or up-regulated (orange) in senescent versus proliferating cells are shown (mean fold change \pm S.D.; $n=3$ ). Right: Plot showing the correlation $\left(\mathrm{R}^{2}\right)$ between actual and predicted passage number based on the methylation levels of six CpGs measured by pyrosequencing (from.

(C) Raw RNA-seq data from all donors and across all three cell types were normalized in silico using the RUVseq package. Left: Box plots show the fluctuation of mRNA expression levels in proliferating ( grey) and senescent cell populations (green) per each donor, before ("raw"; grey outline) and after normalization ("norm"; black outline). Right: Principal component analysis (PCA) plots for each RNA-seq data set in proliferating (grey) and senescent cells (green) per each donor, before (grey outline) and after normalization (black outline). Note that MSC-D1 RNA-seq has twice as many reads as those for D2-4, and thus clusters further away.

(D) Plots showing differentially expressed mRNAs ( $\pm 0.6-\log _{2}$ fold change or more; $\left.P_{\text {adj }}<0.05\right)$ after RNA-seq data normalization for IMR90 mRNA, IMR90 factory RNA, HUVEC, and MSC from two single donors (with the exception of four donors used for MSC). The number of up- (orange) and down-regulated genes (green) is shown in each plot.

(E) Gene ontology (GO) and pathway enrichment analysis for the up- (top) or down-regulated genes (bottom) from each cell type/condition; common GO terms/pathways are displayed in bold.

(F) Shared stimulated GO terms/pathways in senescence. Left: Venn diagram showing genes up-regulated across all cells types. Right: 153 shared up-regulated genes associate with the pathways displayed in the drawn colorcoded network.

(G) Overlap of suppressed genes seen in our and in publicly-available IMR90 RNA-seq data (from Rai et al., 2014). Left: Venn diagram showing genes down-regulated in both datasets. Right: 789 shared down-regulated genes associate with the GO terms/pathways shown in the bar plot.

(H) Validation of gene expression changes in two HUVEC donors using RT-qPCR. Relative fold changes in mRNA levels of selected genes from successively passaged cells ("pro" to "sen") are shown (mean fold change \pm S.E.M.; $n=2$ ). Changes in HMGB1 (pink) and HMGB2 levels (magenta) are highlighted.

(I) Overlap of suppressed (left) or induced (right) genes seen in poly(A) ${ }^{+}$and factory RNA-seq data from two IMR90 isolates. Venn diagram showing genes up-/down-regulated in both datasets; 424 shared down- and 450 upregulated genes associate with the GO terms/pathways listed. 

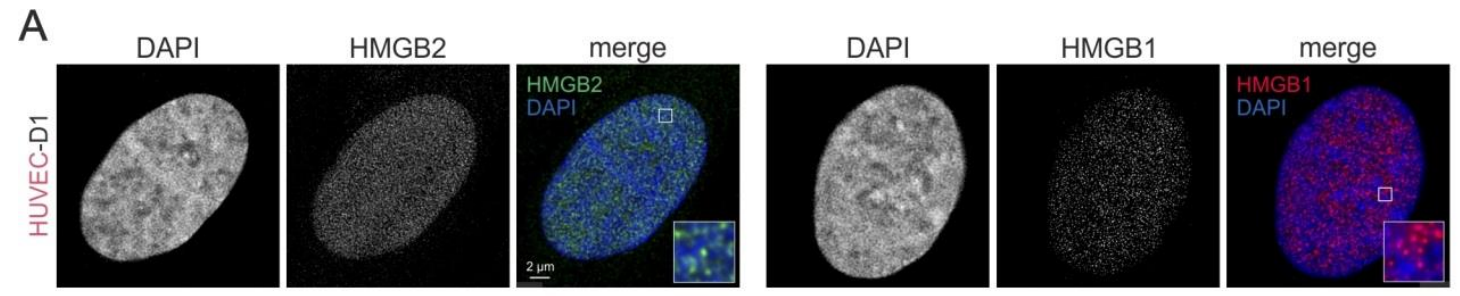

super-resolution imaging

B

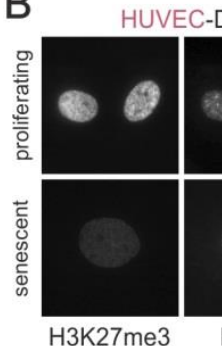

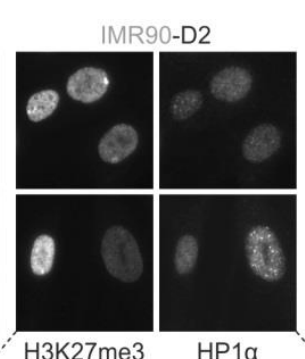

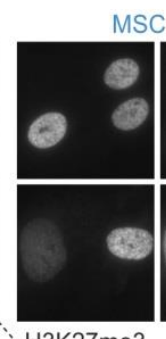

H3K27me3
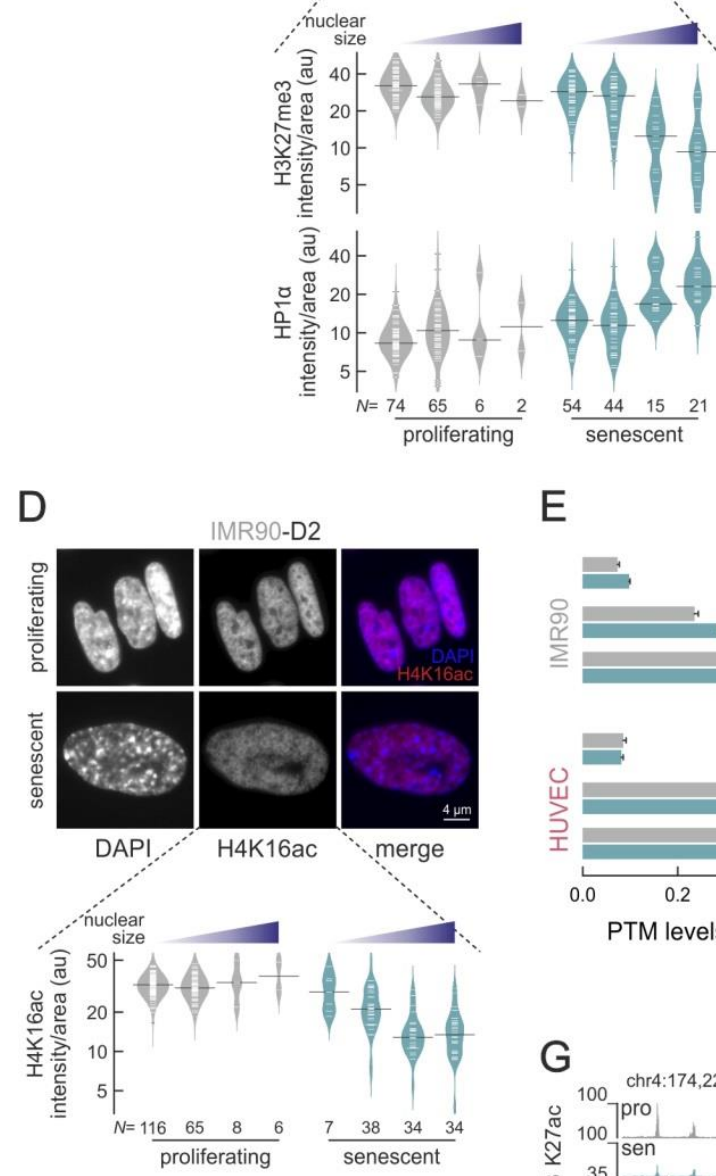

E
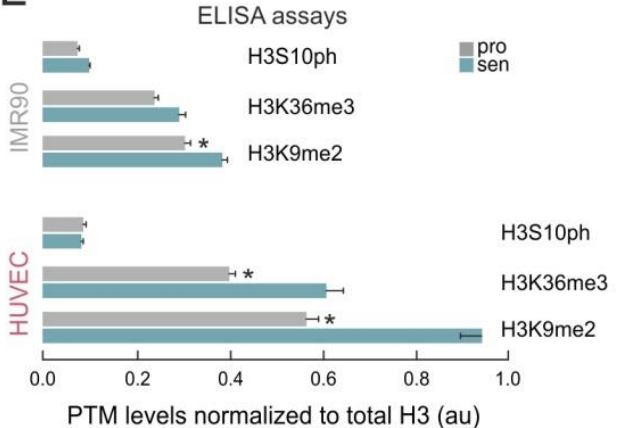

G

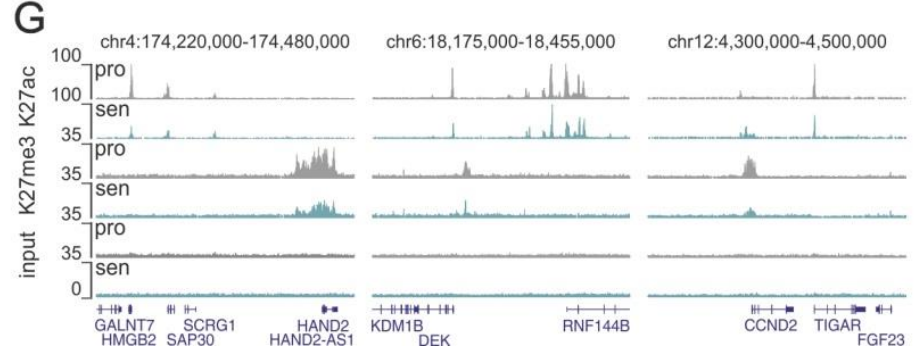

C

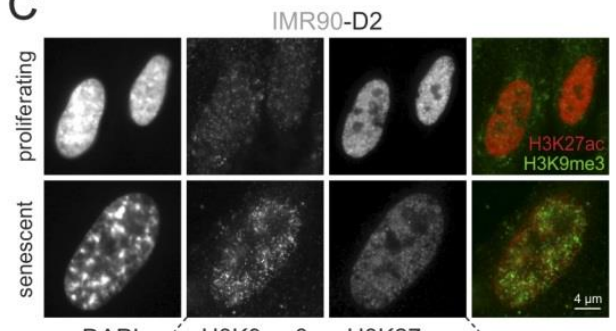

DAPI H3K9me3 H3K27ac merge

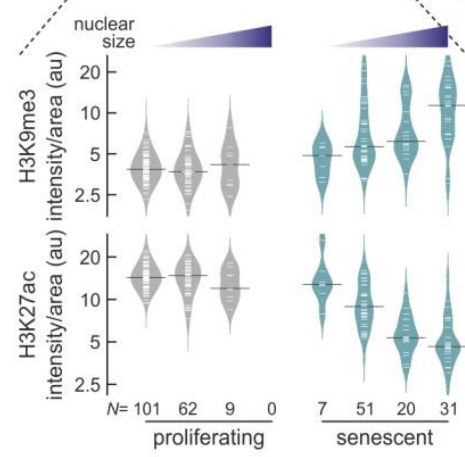

$\mathrm{F}$

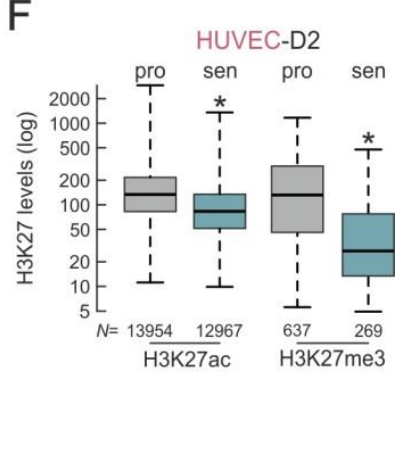


Figure 3.1.S2. Senescence-induced nuclear changes studied at the single-cell level (related to Figure 3.1.3) (A) Super-resolution imaging of HMGB1/B2 nuclear distribution. gSTED images of a representative proliferating HUVEC nucleus immunostained for HMGB2 (top row) and HMGB1 (bottom row).

(B) Widefield images of representative proliferating (top row) and senescent (bottom row) HUVEC, IMR90, and MSC single-donor cells immunostained for H3K27me3 and HP1 $\alpha$. Below: Bean plots show changes in H3K27me3 and HP1 $\alpha$ levels in proliferating (grey) or senescent IMR90 (green) grouped according to increasing nuclear size (from left to right: nuclei smaller than the mean of proliferating cells; between the mean and $1.5 \mathrm{x}$ that of proliferating cells; between $1.5 \mathrm{x}$ and $2 \mathrm{x}$ the mean of proliferating cells; larger than $2 \mathrm{x}$ the mean size of proliferating cells). The total numbers of nuclei measured $(N)$ per group are indicated below each bean plot.

(C) As in panel B, but for IMR90 immunostained for H3K9me3 and H3K27ac.

(D) As in panel B, but for IMR90 immunostained for H3K16ac.

(E) Bar graphs showing normalized levels ( \pm S.D.; $n=4)$ of post-transcriptional histone modifications (PTM) in proliferating (grey) or senescent (green) IMR90 and HUVEC. *: significantly different mean; $P<0.01$, unpaired, two-tailed, Student's t-test.

(F) Box plots showing total (log) signal under H3K27ac (left) and H3K27me3 ChIP-seq peaks (right) from proliferating (grey) and senescent (green) HUVEC. *: significantly different; $P<0.01$, Wilcoxon-Mann-Whitney test. The total numbers of peaks analyzed $(N)$ per condition are indicated below.

(G) Representative browser views showing H3K27ac and H3K27me3 ChIP-seq data from proliferating (grey) and senescent (green) HUVEC along three chromosomal regions. Input data are aligned below. 
A

\begin{tabular}{lcccc}
\multirow{2}{*}{ A } & \multicolumn{4}{c}{ Hi-C sequencing read statistics } \\
\cline { 2 - 6 } samples & $\begin{array}{c}\text { read-pairs } \\
\text { sequenced }\end{array}$ & $\begin{array}{c}\text { \% pairs } \\
\text { mapped }\end{array}$ & $\begin{array}{c}\text { pairs binned/ } \\
\text { processed }\end{array}$ & $\begin{array}{c}\text { cis:trans } \\
\text { read ratio }\end{array}$ \\
\hline HUV-D1 pro $382,431,967$ & 91.81 & $246,141,090$ & $61: 39$ \\
& sen $433,798,275$ & 93.72 & $290,010,130$ & $64: 36$ \\
HUV-D2 & pro $331,260,858$ & 90.52 & $195,085,263$ & $56: 44$ \\
sen $383,019,681$ & 93.19 & $249,439,756$ & $62: 38$ \\
IMR-D1 pro $324,791,121$ & 69.83 & $170,869,508$ & $83: 17$ \\
sen $309,340,828$ & 82.09 & $190,423,483$ & $81: 19$ \\
IMR-D2 pro $365,955,873$ & 67.51 & $172,224,811$ & $84: 16$ \\
& sen $339,556,076$ & 75.48 & $179,744,196$ & $82: 18$ \\
MSC-D1 & pro $290,778,132$ & 80.79 & $170,500,231$ & $78: 22$ \\
sen $343,421,137$ & 80.55 & $205,287,734$ & $80: 20$ \\
\hline
\end{tabular}

\section{B}

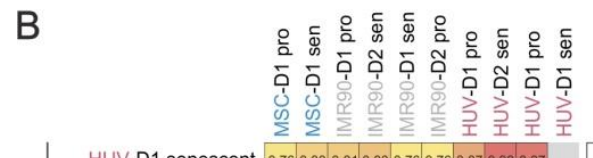

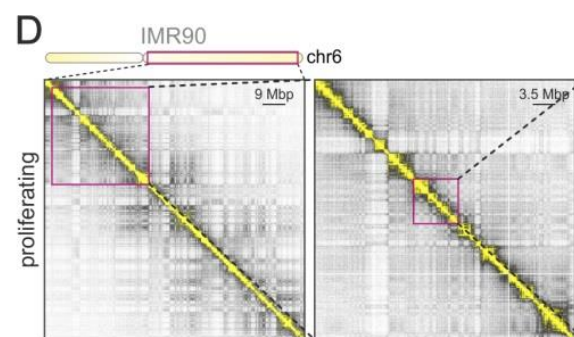
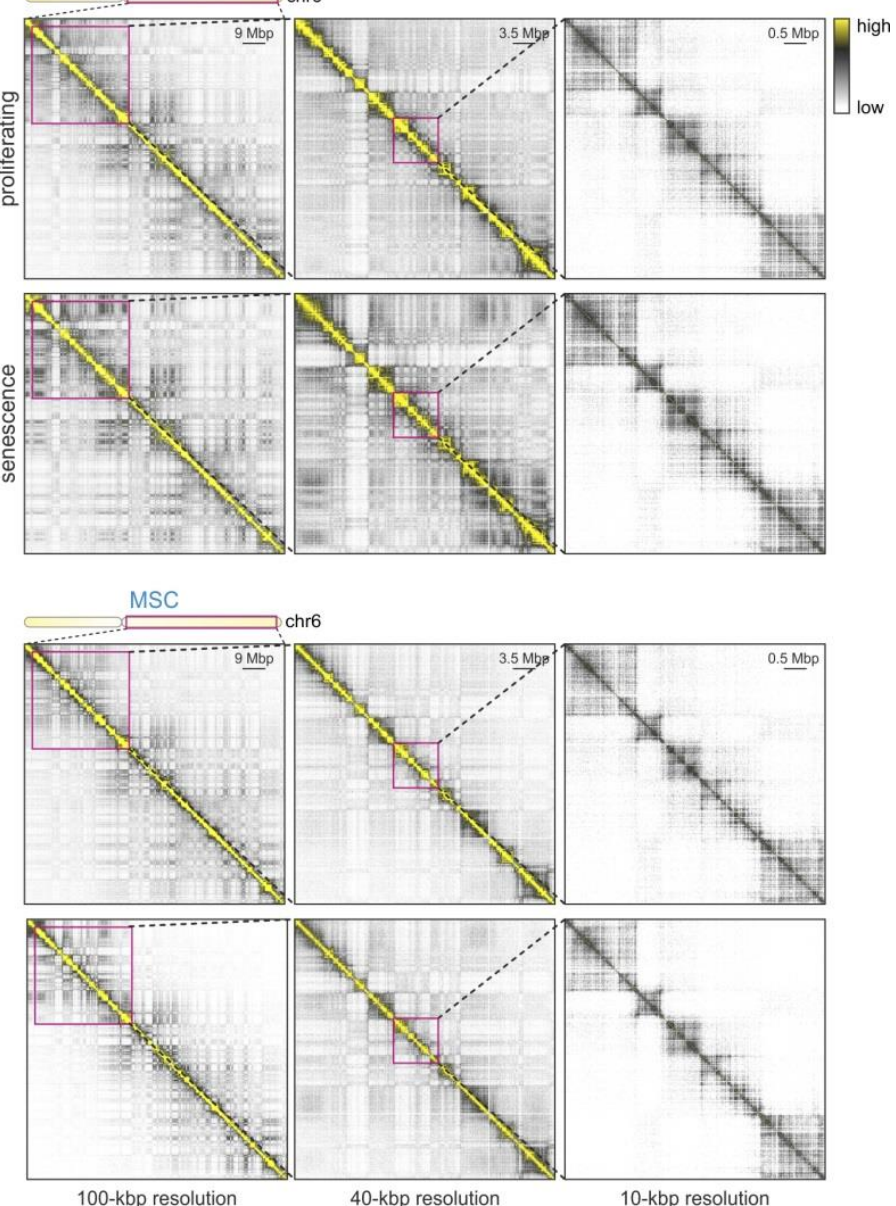

100-kbp resolution

10-kbp resolution

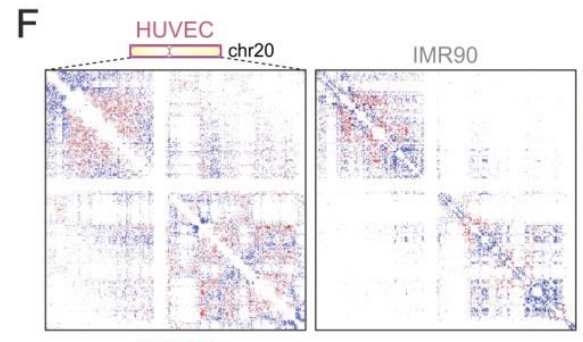

HUVEC
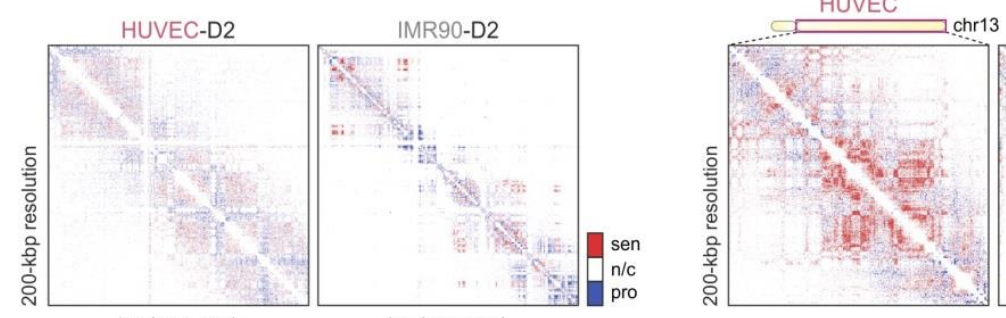

$\log$ (sen-pro)

$\log ($ sen-pro)
IMR90

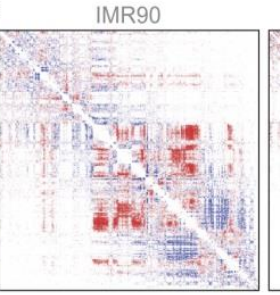

$\log ($ sen-pro)
MSC
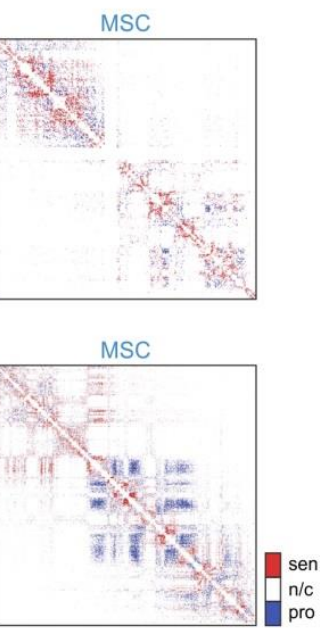

Figure 3.1.S3. Hi-C data generated from proliferating and senescent cells (related to Figure 3.1.4).

(A) Table showing (from left to right) the total number of read pairs generated for each Hi-C library, the per cent of reads mapped, the number of read pairs remaining after binning (in 10-kbp bins) and processing, and the ratio of cis:trans interactions in the final contact matrices.

(B) Heatmap showing correlation values between Hi-C replicas for chromosomes 12 (black squares) and 2 (blue squares) calculated using HiCRep (Yang et al., 2017) and 40-kbp resolution data.

(C) Heatmaps showing increase/decrease (red/blue) in normalized interchromosomal interactions between individual chromosomes from HUVEC and IMR90 data. 
(D) Hi-C heatmaps showing interaction frequencies along the long arm of chromosome 6 (ideogram) in proliferating and senescent IMR90 and MSC at 100-, 40-, and 10-kbp resolution.

(E) Heatmaps show binary increase/decrease (log; red/blue) in normalized Hi-C contact frequency along chromosome 7 (ideogram) for HUVEC and IMR90 from two individual donors/isolates (D1 and D2) at 200-kbp resolution.

(F) As in panel E, but for the long arm of chromosomes 20 and 13 (ideogram) from senescent versus proliferating HUVEC, IMR90, and MSC at 200-kbp resolution. 

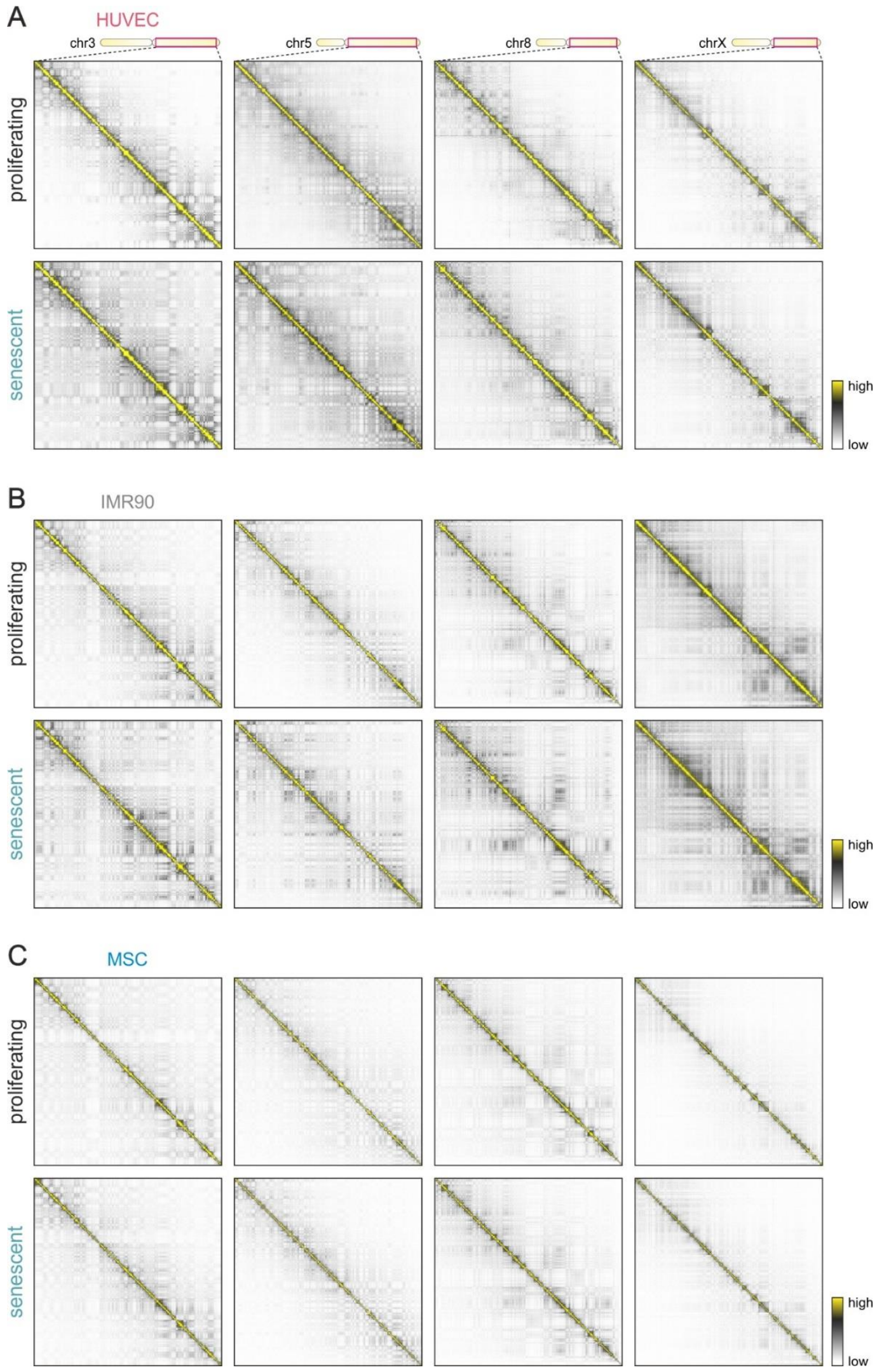

Figure 3.1.S4. Hi-C contact maps from proliferating and senescent cells (related to Figure 3.1.4).

$\mathrm{Hi}-\mathrm{C}$ heatmaps showing contact frequencies along the long arms of chromosomes 3, 5, 8, and $\mathrm{X}$ (ideograms) from proliferating/senescent HUVEC (A), IMR90 (B), and MSC (C) at 40-kbp resolution. 
A
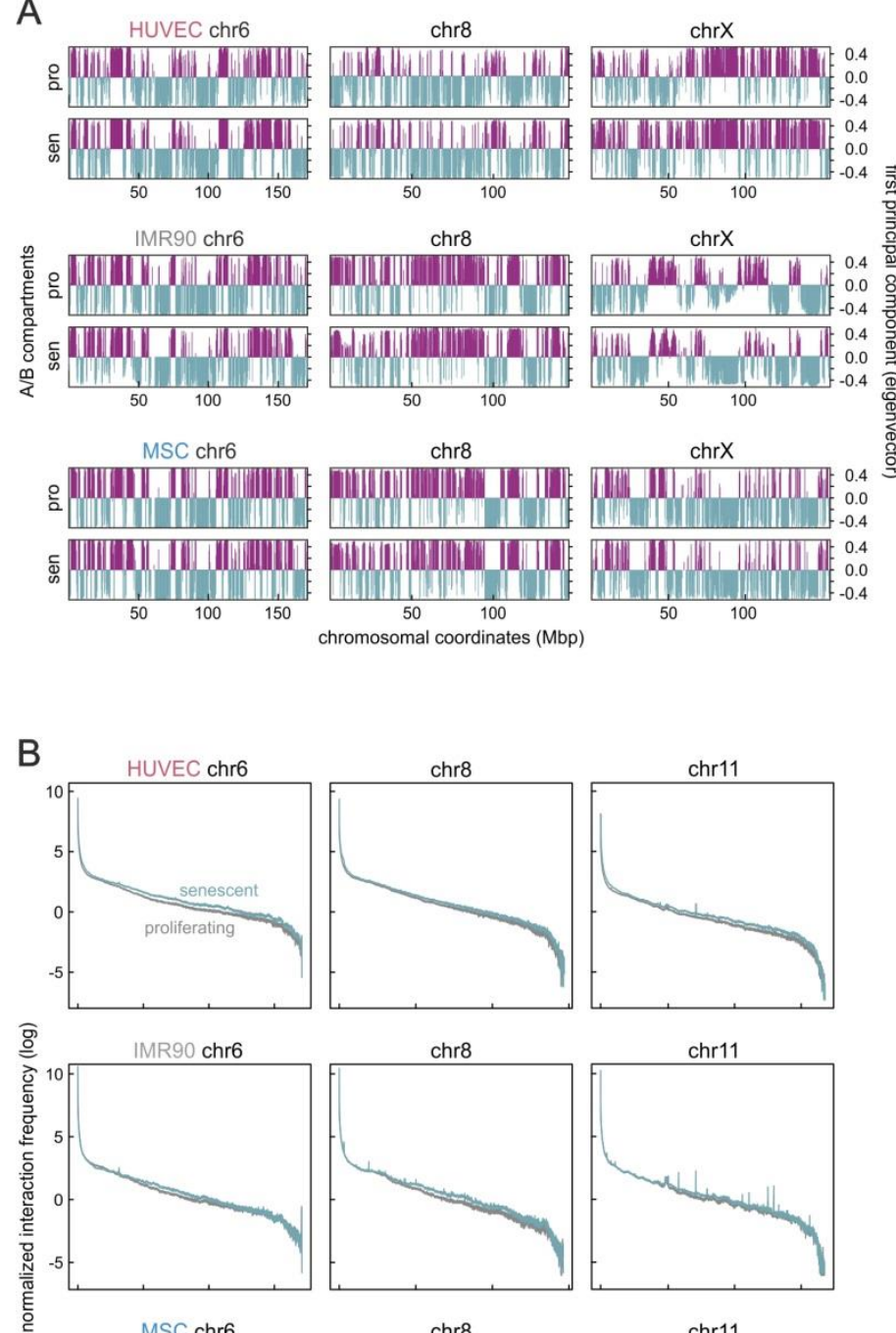

chr8
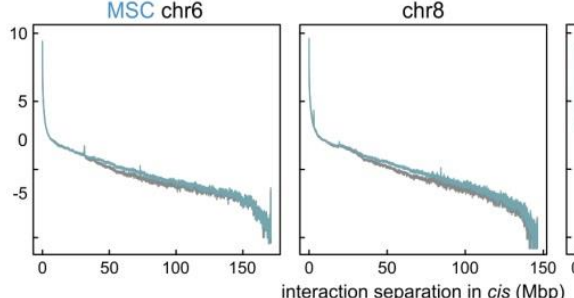
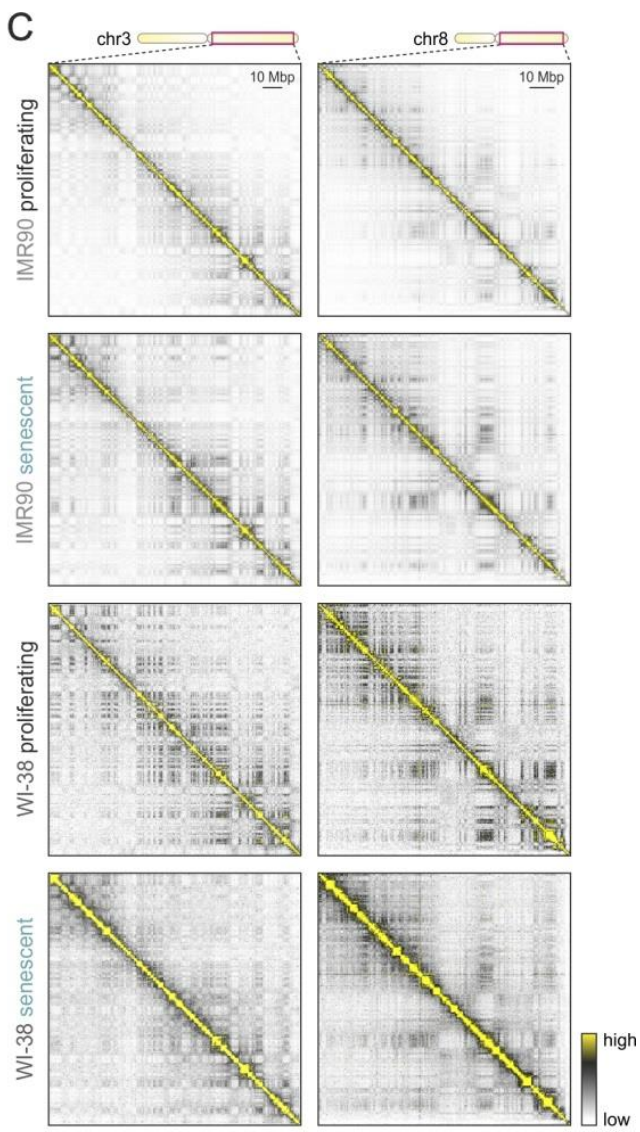

D

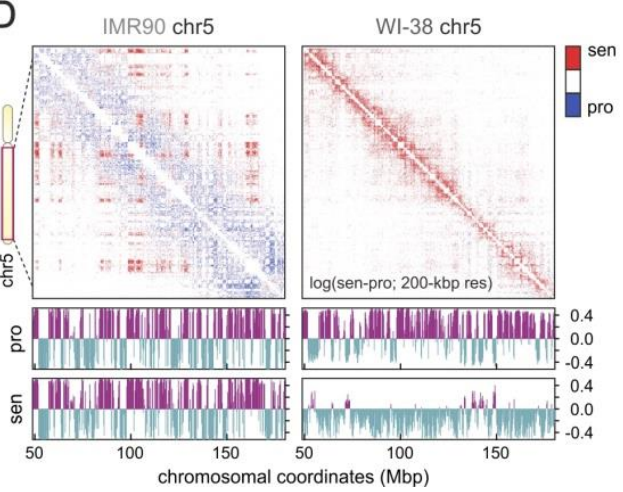

Figure 3.1.S5. Changes in chromosome-wide interactions upon senescence entry and comparison to Hi-C data from "deep" senescence (related to Figure 3.1.4).

(A) Graphs showing switching between A (purple) and B compartments (green) along chromosomes 6, 8, and X in proliferating (top row) and senescent (bottom row) HUVEC, IMR90, and MSC.

(B) Line plots showing normalized Hi-C contact frequency $(\log )$ with distance along chromosomes 6, 8, 11, and 16 from proliferating (grey line) and senescent (green line) HUVEC, IMR0, and MSC.

(C) Hi-C heatmaps showing interaction frequencies along the long arm of chromosomes 3, 5, and 8 (ideograms) from proliferating/senescent IMR90 (top) and WI-38 (bottom) at 40-kbp resolution.

(D) Heatmaps show increase/decrease (log; red/blue) in normalized Hi-C interaction frequency along the long arm of chr5 (ideogram) from senescent versus proliferating IMR90 and WI-38 cells. Graphs showing switching between A (purple) and B compartments (green) along the long arm of chr5 in proliferating (top row) and senescent (bottom row) IMR90, and WI-38 are aligned below. 


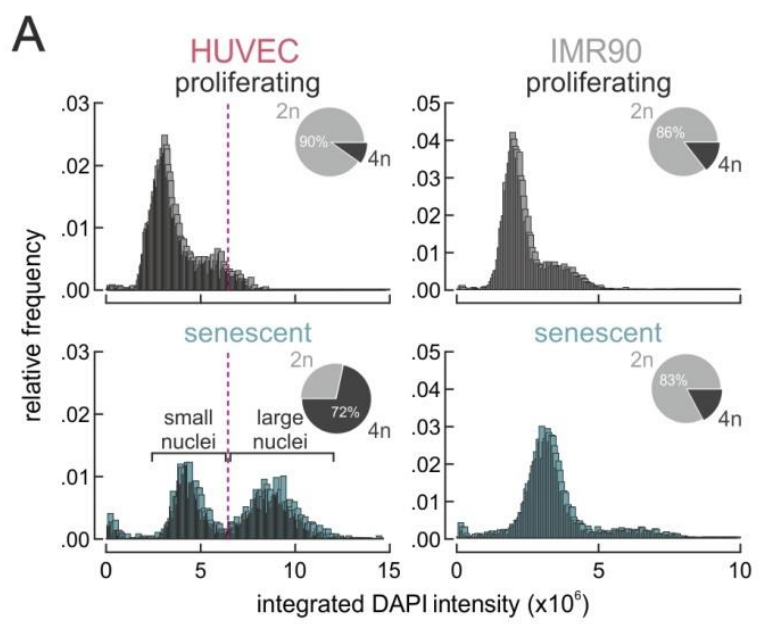

B HUVEC

IMR90

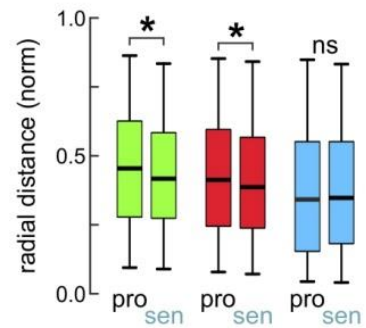

C

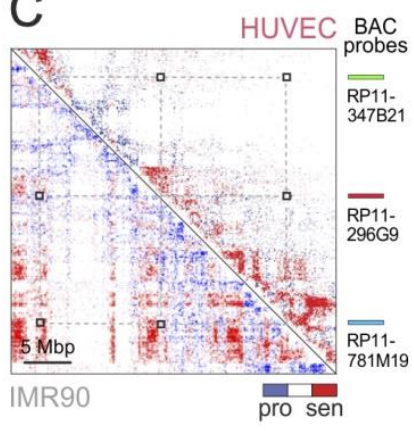

D

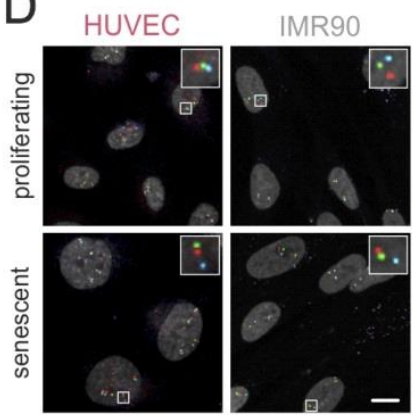

$E$

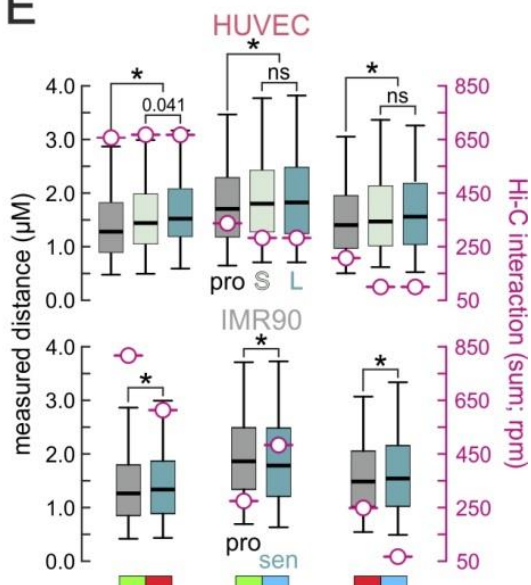

$\mathrm{F}$

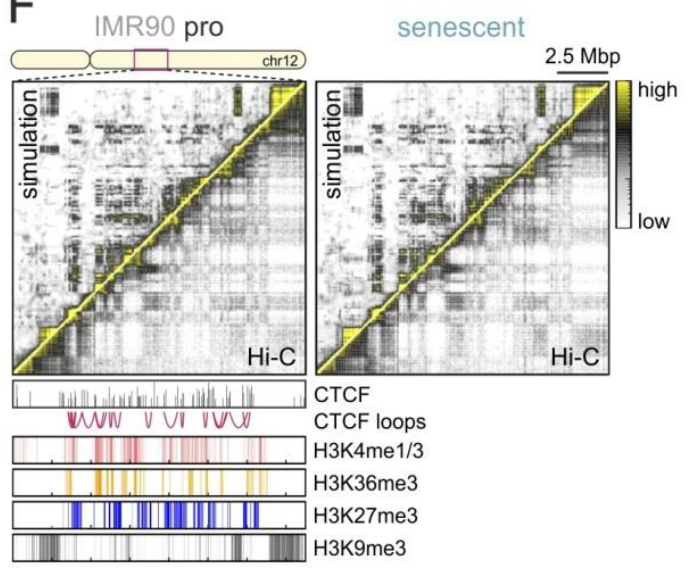

G
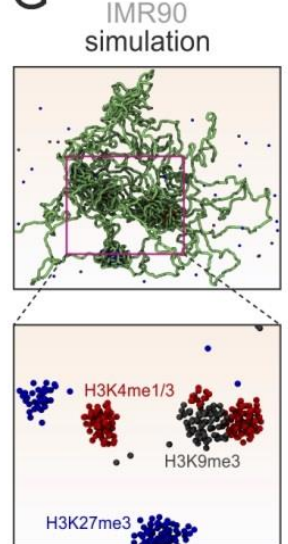

$\mathrm{H}$

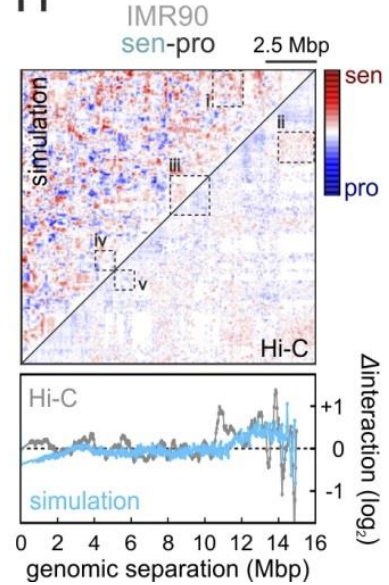

Figure 3.1.S6. 3D-DNA FISH and modeling of genomic organization in senescence (related to Figure 3.1.4). (A) Plots showing the distribution of DAPI intensity in proliferating and senescent HUVEC (left) and IMR90 nuclei (right) as revealed by high throughput 3D-DNA FISH image analysis. Note the bimodal distribution of senescent HUVEC nuclear sizes that allowed us to group them into smaller and larger ones. Insets: Pie charts show the percent of diploid $(2 n)$ or tetraploid $(4 n)$ nuclei in each population.

(B) Box plots showing the normalized radial positions $(0=$ central to the nucleus; $1=$ peripheral $)$ of the three BACs (green, red, blue) used as probes in 3D-DNA FISH in proliferating ("pro") or senescent ("sen") HUVEC and IMR90. *: significantly different; $P<0.05$, Wilcoxon-Mann-Whitney test. 
(C) Heatmaps show binary increase/decrease (red/blue) in normalized Hi-C contact frequencies along a 30-Mbp subregion chromosome 12 (ideogram) in HUVEC and IMR90 at 200-kbp resolution. The relative positions of the BAC clones used as DNA FISH probes ( $g r e e n$, red, and blue), and their interactions are indicated. Bottom: a zoomin on the changing Hi-C interactions between each BAC probe pair in a 1-Mbp space.

(D) Representative images of HUVEC (left) and IMR90 nuclei (right) hybridized with the three BAC probes (colours as in panel A). Bar: $6 \mu \mathrm{m}$.

(E) Box plots showing the shift in measured 3D-distances in proliferating (grey) and senescent (green) HUVEC and IMR90, and their respective changes in Hi-C interaction frequencies (magenta ovals) for each probe pair (bottom). Note how HUVEC senescent cells are grouped according to their nuclear size ( $\mathrm{S}$, small; L, large) like in panel A. *: significantly different; $P<0.01$, Wilcoxon-Mann-Whitney test.

(F) Comparison of in silico ("simulation") and experimental contact heatmaps ("Hi-C") for the 15-Mbp subregion of chromosome 12 (ideogram) used in simulations in both the proliferative and senescent state. The positions of CTCF, H3K4me1/3, H3K36me3, H3K27me3, and H3K9me3 ChIP-seq peaks are also aligned below.

(G) Snapshots of individual simulation runs using the parameters from the proliferating-cell model; the chromatin fiber (green) and CTCF proteins (spheres) are shown. Magnification: a subregion of the simulated system is shown, in the absence of the chromatin fiber, to visualize active ( $r e d)$, inactive (blue) and heterochromatic (black) protein clusters.

(H) Heatmaps show increase/decrease (red/blue) in normalized Hi-C interaction frequencies in the 15-Mbp modelled subregion of chromosome 12 at 100-kbp resolution. Representative regions where contact changes are similar (rectangles $i$ - $i i$ and $i v-v$ ) or differ (rectangle iii) are demarcated. Below: plot showing the changes in interactions $\left(\log _{2}\right)$ from in silico (light blue) or experimental data (grey) along the subregion. 


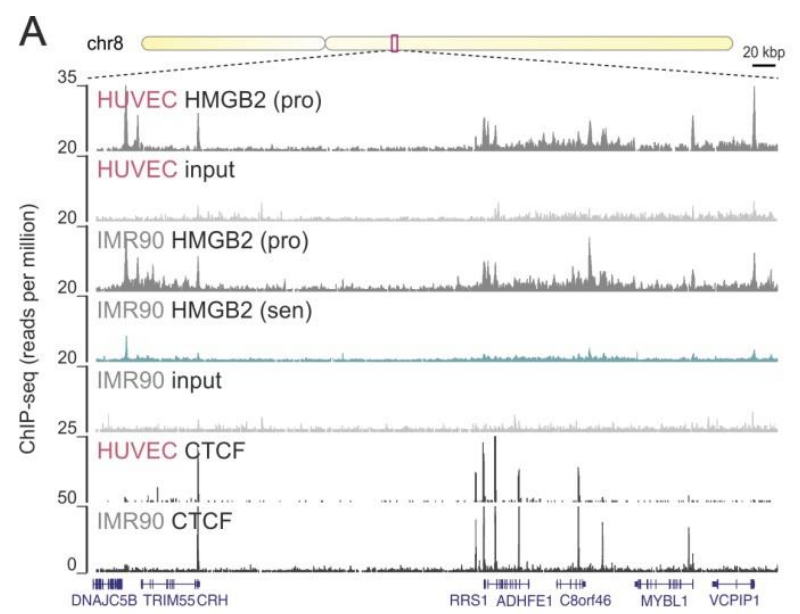

D
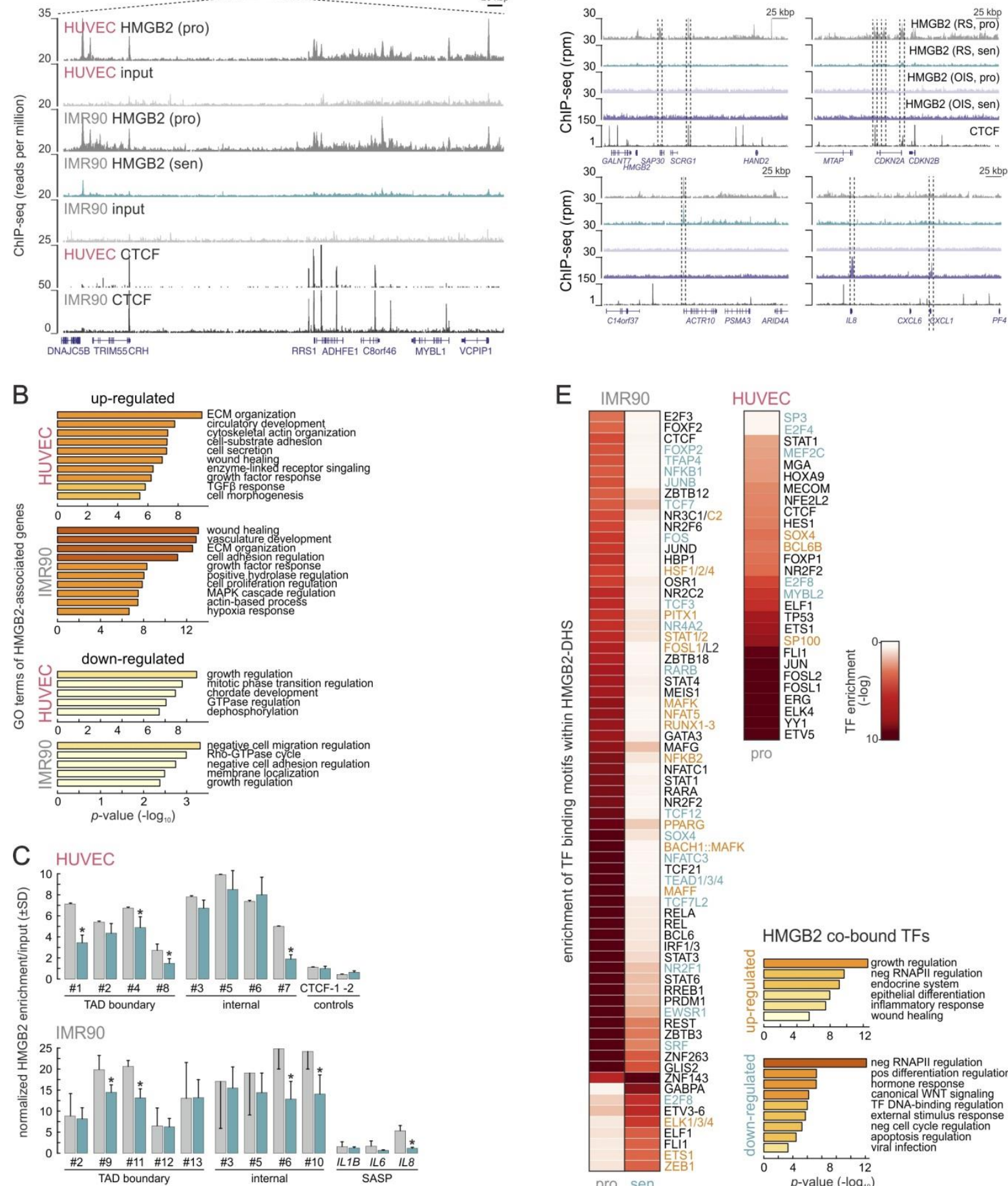

E
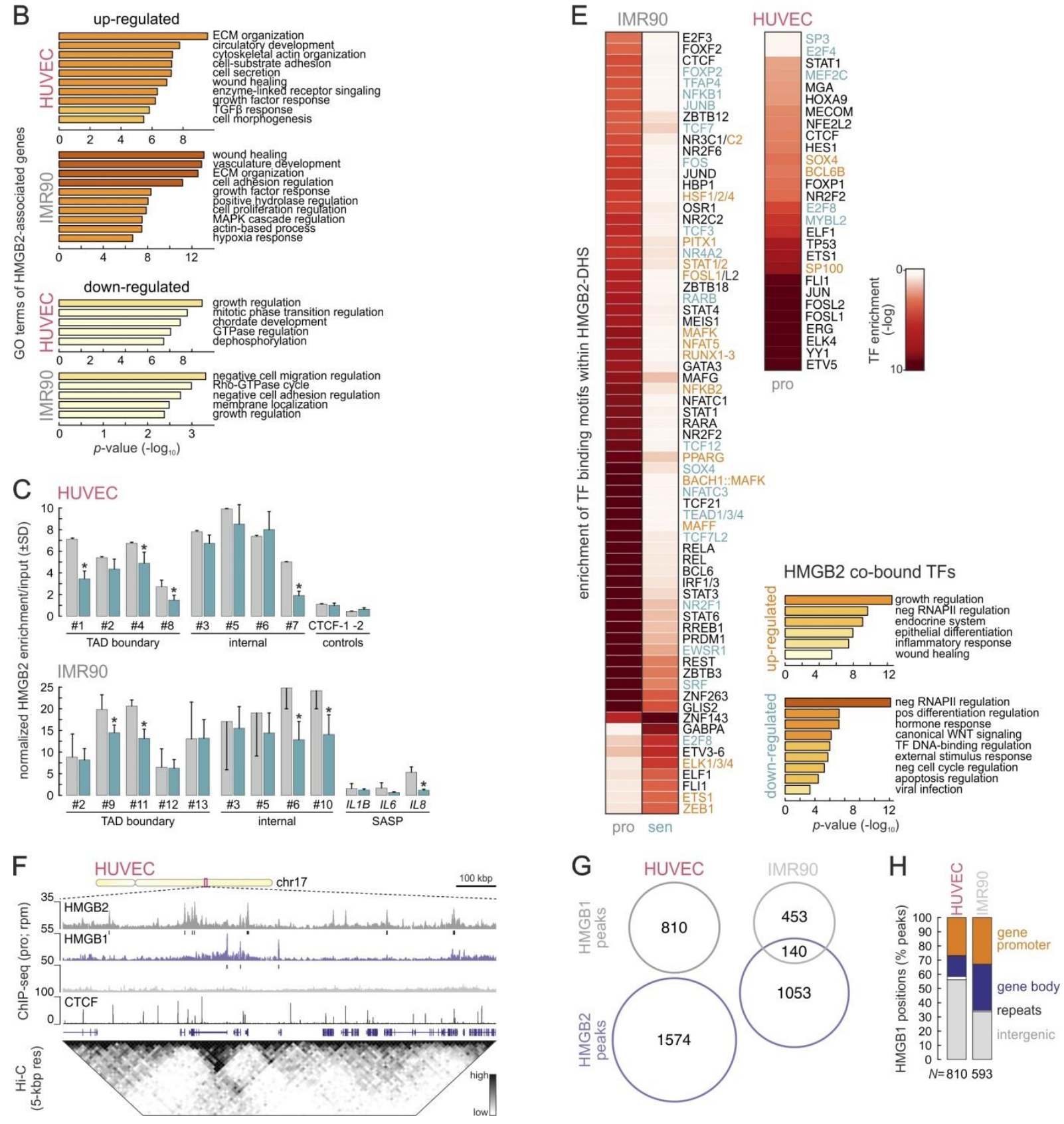
Figure 3.1.S7. Analysis of HMGB1/B2 chromatin-binding properties (related to Figure 3.1.5).

(A) Genome browser view of HMGB2 ChIP-seq profiles from proliferating HUVEC and proliferating/ senescent IMR90 along a subregion of chromosome 8 (ideogram); CTCF ChIP-seq profiles and Refseq gene models are also aligned below.

(B) Gene ontology (GO) and pathway enrichment analysis for the up- (top) or down-regulated genes (bottom) bound by HMGB2 in HUVEC or IMR90.

(C) HMGB2 ChIP-qPCR ( \pm S.D.; $n=3)$ was used to interrogate changes in binding between proliferating ( grey) and senescent cell populations (green). HMGB2 peaks are grouped into those positioned at TAD boundaries and those internal to TADs; CTCF-bound sites and SASP genes serve as controls.

(D) Browser views showing representative HMGB2 peaks from proliferating (grey) and replicatively-senescent IMR90 (green) or from proliferating (light purple) and oncogene-induced senescent WI-38 (dark purple). Top row: HMGB2 peaks lost in RS, but are not at all picked up in OIS; bottom left: an HMGB2 peak persisting upon RS, but again not picked up in OIS; bottom right: two OIS-specific HMGB2 peaks at the SASP genes IL 8 and CXCL1 are not present in RS, despite activation of both loci.

(E) Heat maps showing known transcription factor (TF) binding motif enrichment (log scale) within the DNasehypersensitive sites (DHS) under HMGB2 peaks in IMR90 (left) or HUVEC (top right); TFs up-/down-regulated upon senescence are colored orange and green, respectively. Enriched GO terms/pathways of up- or downregulated TFs co-bound by HMGB2 are shown (bottom right).

(F) A browser view of HMGB2/B1 ChIP-seq profiles from proliferating HUVEC along a subregion of chromosome 17; CTCF ChIP-seq and 5-kbp resolution Hi-C data (Rao et al., 2014) are aligned below.

(G) Venn diagram showing the overlap between HMGB2 (grey) and HMGB1 peaks (purple) detected in proliferating HUVEC and IMR90.

(H) Bar plots showing the percent of HMGB1 ChIP-seq peaks from HUVEC and IMR90 overlapping gene promoters (orange), gene bodies (blue), repeats (white), or intergenic regions (grey). The total number of peaks $(N)$ per dataset is displayed below. 


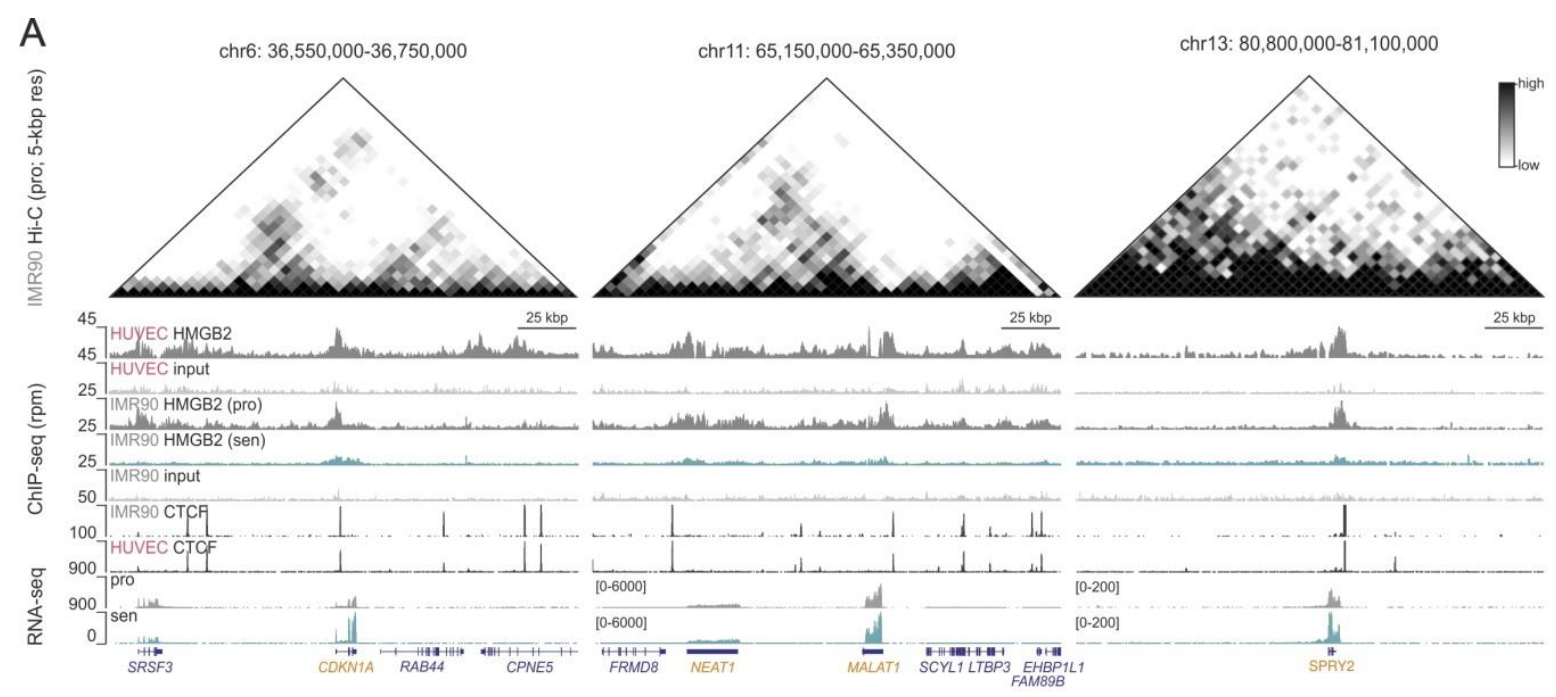

B

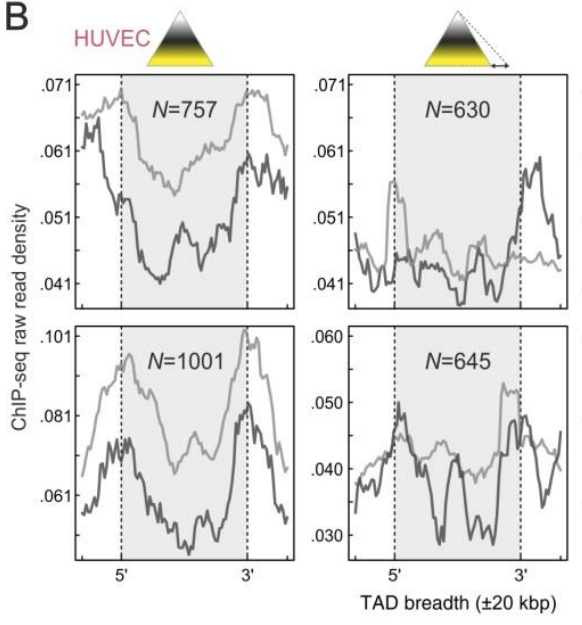

C
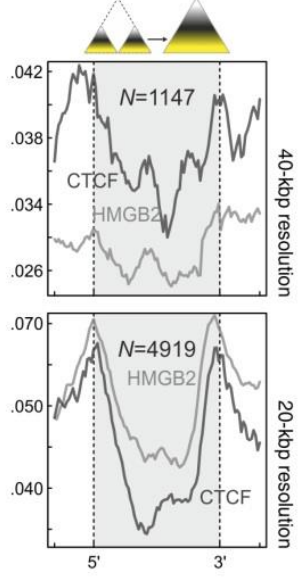

D
HUVEC topologically-associating domains
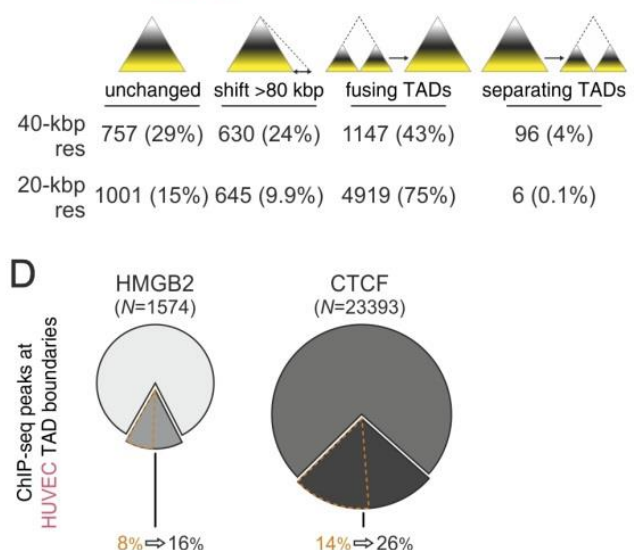

E

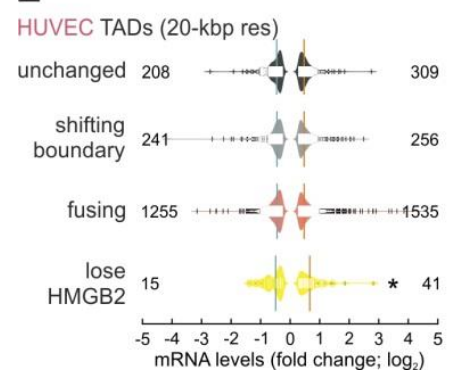

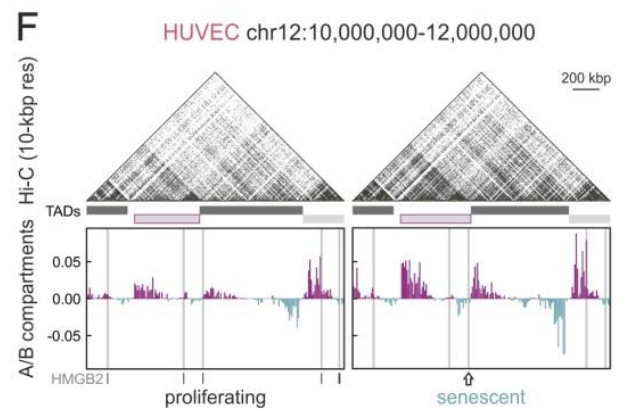

G

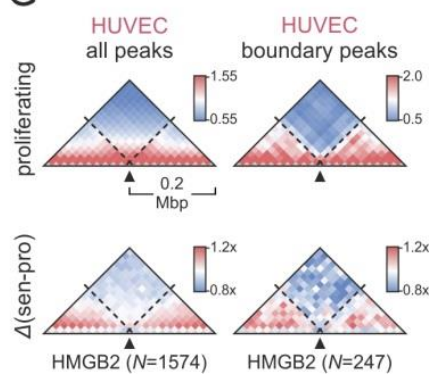

Figure 3.1.S8. Relative positioning of HMGB2 in respect to TAD boundaries (related to Figure 3.1.5 and S6).

(A) Genome browser views of HMGB2 ChIP-seq profiles from proliferating HUVEC and proliferating/ senescent IMR90 along the indicated regions of chromosomes 6, 11, and 13. 5-kbp resolution IMR90 Hi-C data (Rao et al., 2014), and CTCF ChIP-seq, proliferating/senescent RNA-seq profiles, and Refseq gene models are aligned above and below, respectively.

(B) Meta-plots showing the distribution of HMGB2 (grey) and CTCF ChIP-seq signal (black) along HUVEC TADs ( $\pm 20 \mathrm{kbp}$ ) that do not change (left), shift one boundary by $>80 \mathrm{kbp}$ (middle), or fuse into one larger TAD (right). TADs identified using 40- or 20-kbp Hi-C data and the total numbers of TADs in each group $(\mathrm{N})$ are shown.

(C) The number of TADs (percentages in brackets) that remain unchanged, shift one boundary by $>80 \mathrm{kbp}$, fuse into one larger TAD, or separate into smaller TADs upon senescence are listed for the two resolution studied here. 
(D) Pie charts showing the percent of HMGB2 (light grey) and CTCF (dark grey) that overlap HUVEC TAD boundaries called at 40- (orange dashed line) and 20-kbp resolution (solid line).

(E) Bean plots showing $\left(\log _{2}\right)$ fold changes in mRNA levels of differentially-expressed genes contained within TADs with unchanged, shifting boundaries or fusing, or at boundaries that lose HMGB2 upon senescence entry. The total numbers of up-/down-regulated genes $(N)$ are shown. *: significantly different; $P<0.01$, Wilcoxon-MannWhitney test.

(F) Graphs showing switching between A/B compartments along a 2-Mbp subregion of chromosome 12 in proliferating (left) and senescent (right) HUVEC at 10-kbp resolution.

(G) Top: Heatmaps showing normalized interaction frequencies in the $0.4 \mathrm{Mbp}$ around all HMGB2 peaks or around those at TAD boundaries in proliferating HUVEC. Bottom: Heatmaps showing changes in interaction frequencies in the $0.4 \mathrm{Mbp}$ around all or boundary HMGB2 peaks (at 20-kbp resolution) between senescent/proliferating HUVEC. 

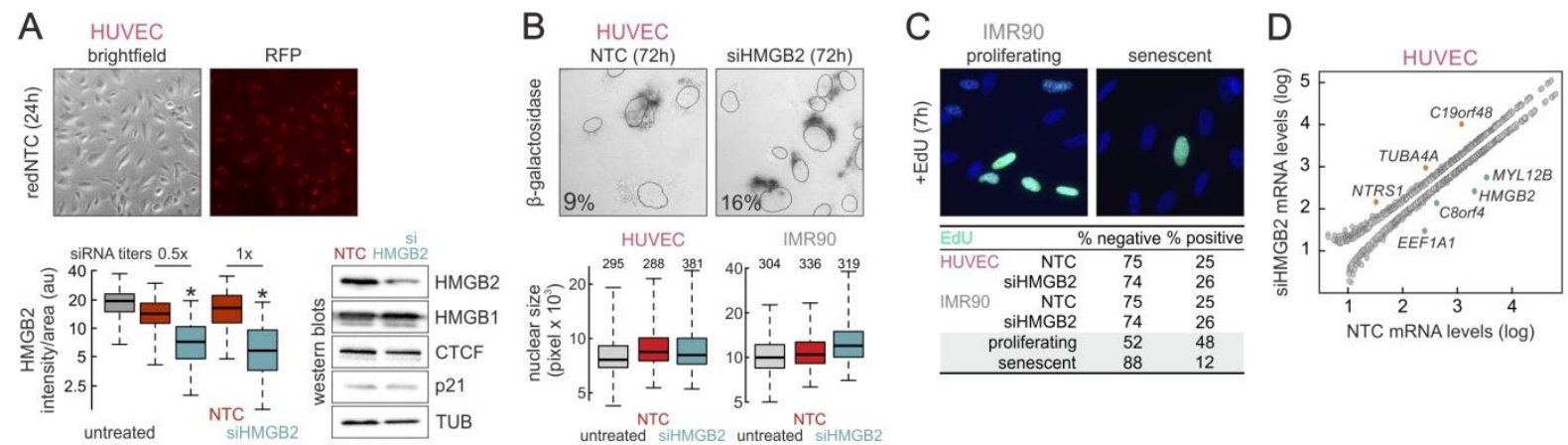

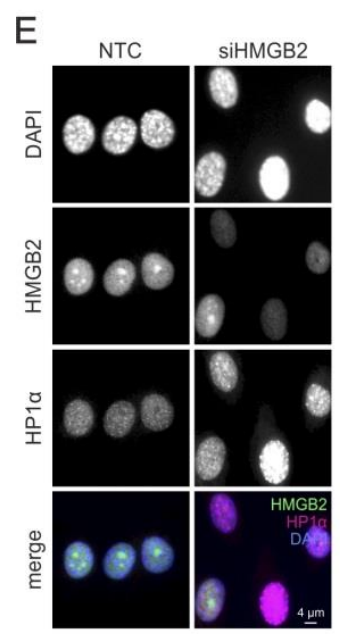

widefield microscope
$\mathrm{F}$

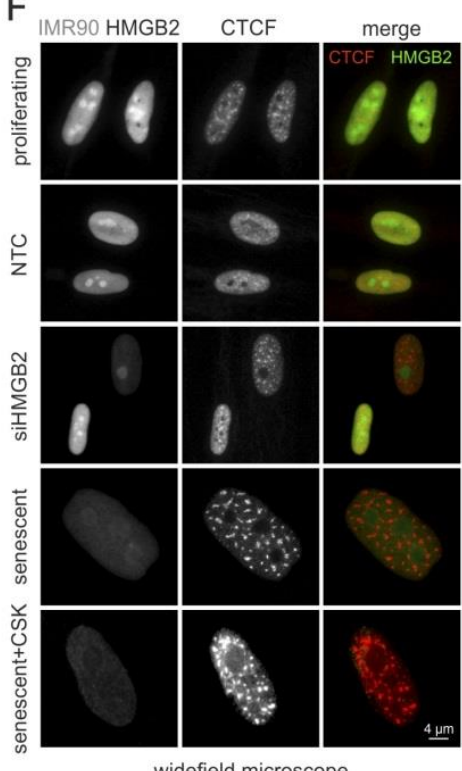

widefield microscope
G

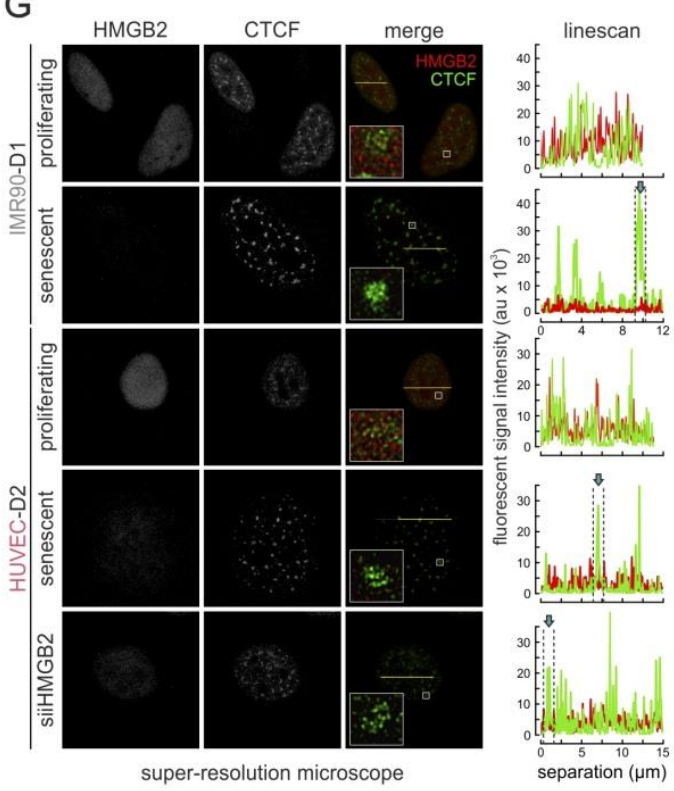

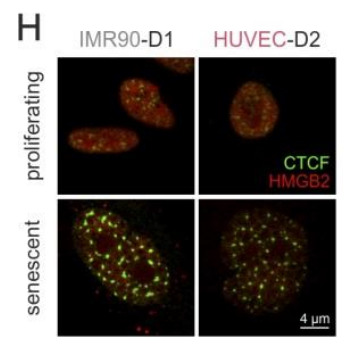

confocal microscope

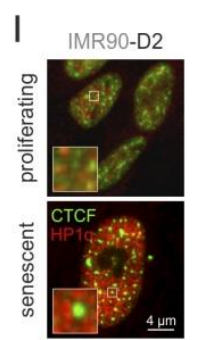

widefield microscope

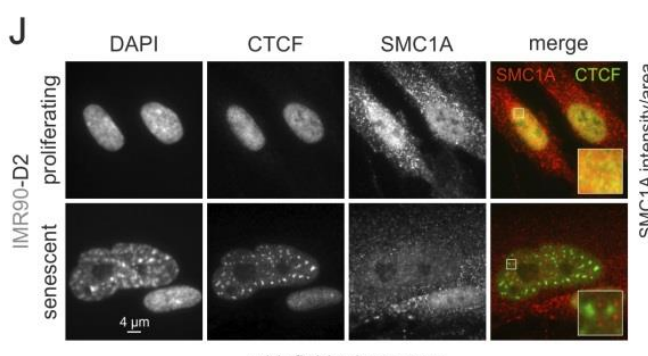

widefield microscope
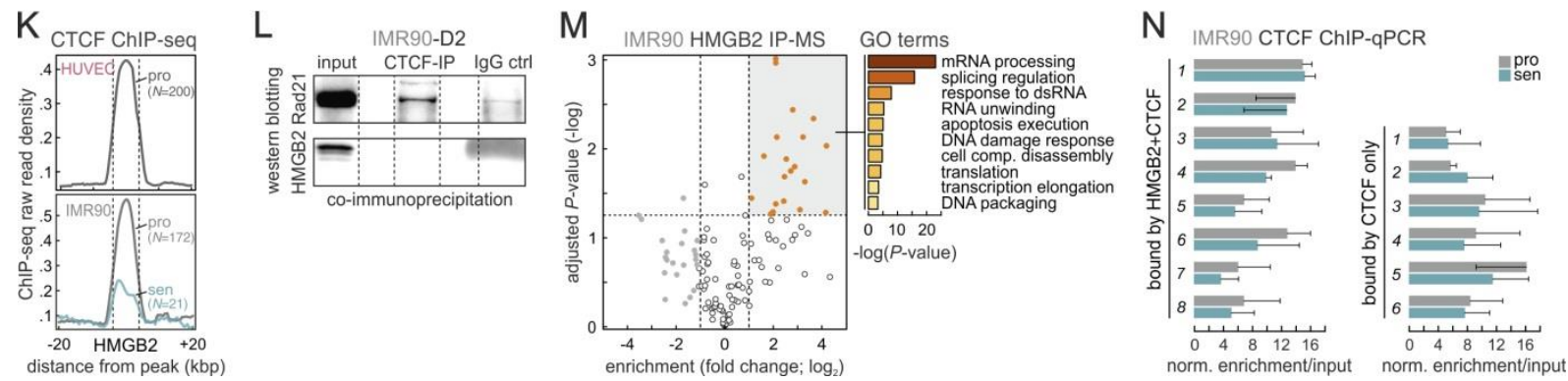
Figure 3.1.S9. Effects of $H M G B 2$ knock-down on primary human cells (related to Figure 3.1.6).

(A) Top: Typical images of proliferating HUVEC treated with self-delivering, fluorescently-labeled, non-targeting siRNAs ("redNTC") for $24 \mathrm{~h}$. Bottom left: Box plots showing HMGB2 levels immuno-detected in control ("NTC") or knock-down HUVEC ("siHMGB2") treated with different siRNA titers. *: significantly different; $P<0.01$, Wilcoxon-Mann-Whitney test. Bottom right: Western blots of HMGB1, HMGB2, CTCF, and p21 in NTC and knock-down HUVEC; tubulin levels provide a control.

(B) Top: Typical images of control ("NTC") and knock-down HUVEC ("siHMGB2") stained for HMGB2 and $\beta$ galactosidase activity; percent of $\beta$-gal positive cells are indicated. Bottom: Box plots showing distribution in nuclear size for untreated, NTC, or siHMGB2 HUVEC (top) and IMR90 (bottom).

(C) Top: Typical images of proliferating (left) and senescent IMR90 (right) grown in the presence of EdU (green) for $7 \mathrm{~h}$; DAPI staining was used to visualize nuclei. Bottom: Table showing percentages of EdU-negative and EdUpositive cells in NTC- or siHMGB2-treated HUVEC/IMR90; the percentages recorded in proliferating/senescent IMR90 serve as a control.

(D) Scatter plot showing differentially-expressed mRNAs (at least $\pm 0.3-\log _{2}$ fold change; $P<0.05$ ) after RNA-seq data normalization for knock-down ("siHMGB2") versus control HUVEC ("NTC") from two replicates. Genes most up- (orange) or down-regulated (green) are indicated.

(E) Widefield images of representative control (top), and HMGB2-knockdown HUVEC nuclei (bottom) immunostained for HMGB2 and HP1 $\alpha$.

(F) Representative wide-field images of proliferating (top row), control (second row from top), knock-down (third row from top), senescent (penultimate row), or senescent and pre-extracted with CSK IMR90 (bottom row) immunostained for HMGB2 and CTCF.

(G) gSTED images of proliferating, senescent, or siHMGB2 IMR90 and HUVEC immunostained for HMGB2 (left column) and CTCF (middle column); characteristic regions are magnified (inset). Line scans (far right) provide profiles of CTCF (green curve) and HMGB2 fluorescence (red curve) across a 10-15 $\mu \mathrm{m}$ section indicated in the "merge" panel (yellow line) with SICCs are indicated (arrows).

(H) Confocal imaging of representative proliferating (top row) and senescent (bottom row) IMR90 and HUVEC cells immunostained for HMGB1/B2 and CTCF.

(I) Representative wide-field images of proliferating (left) and senescent IMR90 (right) showing no overlap between immunostainings for HP1 $\alpha$ and CTCF foci.

(J) Left: Representative wide-field images of proliferating (top row) and senescent IMR90 (bottom row) immunostained for SMC1A and CTCF. Right: Box plots showing SMC1A levels immunodetected in proliferating (grey), senescent (blue), and SICC-positive senescent IMR90 (green). Bar: $4 \mu \mathrm{m}$.

(K) Line plot showing distribution of CTCF ChIP-seq signal in the $40 \mathrm{kbp}$ around HMGB2 peaks from proliferating HUVEC and IMR90 (grey) or senescent IMR90 (green). The number of overlapping peaks $(N)$ per cell types is indicated.

(L) Representative western blot of Rad21 and HMGB2 in co-immunoprecipitation experiments with CTCF; control co-IPs using non-specific IgG antisera provide a control.

(M) Volcano plot showing mass-spectrometry data for the relative enrichment of proteins interacting with HMGB2 (orange dots; $N=67$ ) versus IgG controls (grey dots denote contaminants) using $P$-value $<0.05$ and fold change $\left(\log _{2}\right)>1$ as cutoffs. The 10 most enriched GO terms associated with HMGB2 interaction partners are listed (right). $(\mathrm{N})$ CTCF ChIP-qPCR ( \pm S.D.; $n=3$ ) reveals little change in binding between proliferating (grey) and senescent IMR90 (green). CTCF peaks are grouped into those overlapping ("HMGB2+CTCF") or not overlapping HMGB2bound sites ("CTCF only"). 
A HUVEC-D1 NTC

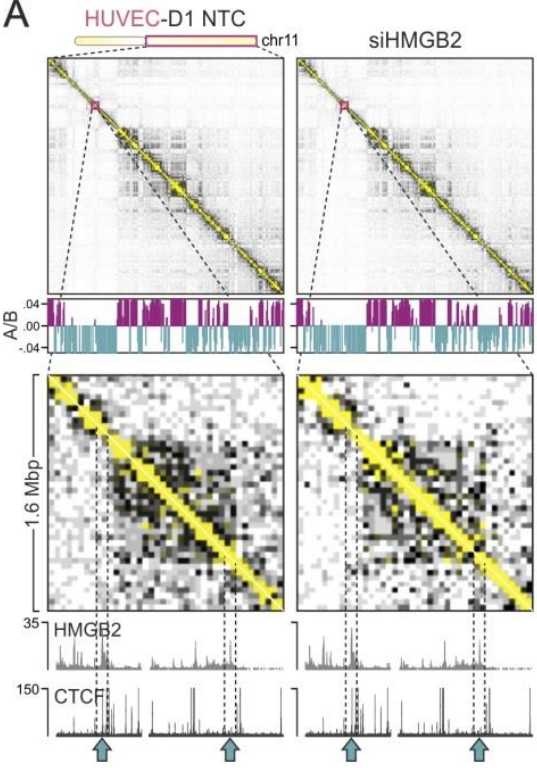

D

\begin{tabular}{|c|c|c|c|c|c|}
\hline & $\begin{array}{l}\text { total read } \\
\text { pairs }\end{array}$ & $\begin{array}{l}\% \text { mapped } \\
\text { read pairs }\end{array}$ & $\begin{array}{l}\text { interaction } \\
\text { pairs (cis) }\end{array}$ & $\begin{array}{l}\text { reads in loop } \\
\text { anchors (cis) }\end{array}$ & $\begin{array}{l}\text { filtered } \\
\text { loops (cis) }\end{array}$ \\
\hline pro & $156,578,197$ & 66.9 & $5,367,729$ & $23,110,588$ & 2,903 \\
\hline sen & $156,140,941$ & 62.8 & $2,068,577$ & $21,825,041$ & 1,398 \\
\hline 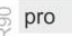 & $175,893,621$ & 57.3 & $1,108,942$ & $14,808,240$ & 1,453 \\
\hline sen & $119,605,102$ & 67.7 & $1,429,210$ & $11,307,054$ & 756 \\
\hline
\end{tabular}

F HUVEC CTCF loops

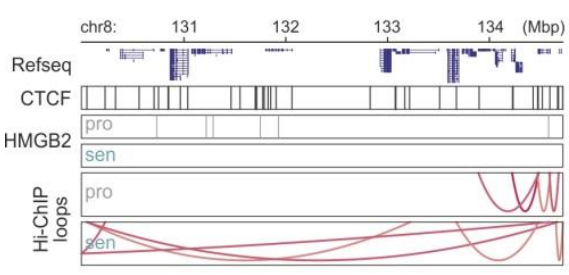

IMR90 CTCF loops
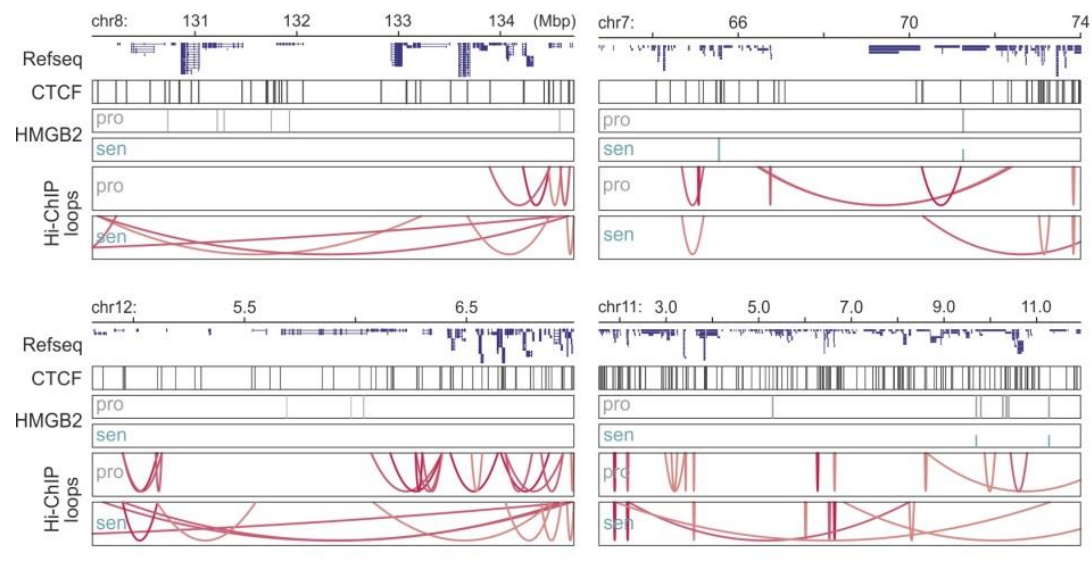

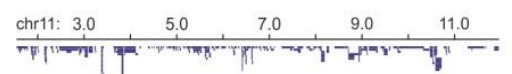


B HUVEC/IMRgo
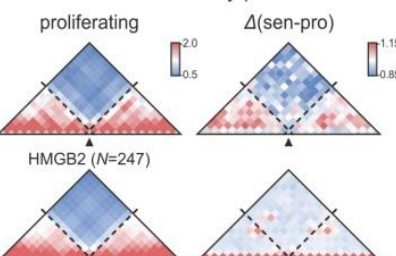

$\operatorname{CTCF}(\hat{N}=2661)$
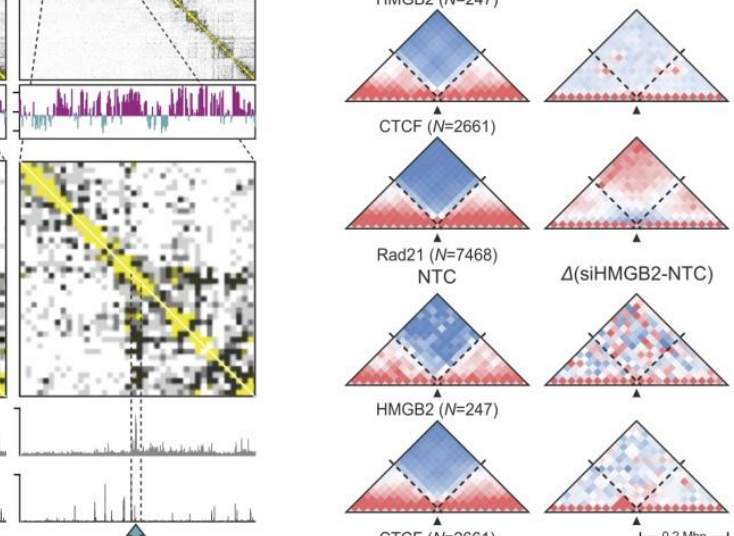

CTCF $(N=2661)$

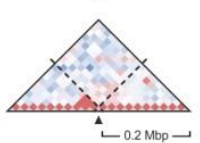

C HUVEC / IMR9O all peaks

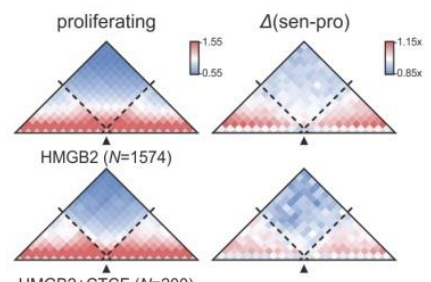

HMGB2+CTCF $(N=200)$
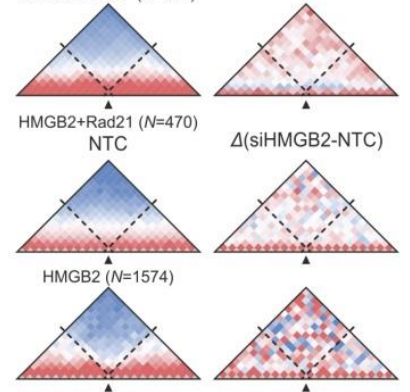

HMGB2+CTCF $(N=200)$

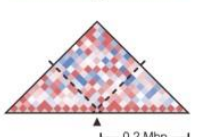

G gene expression changes

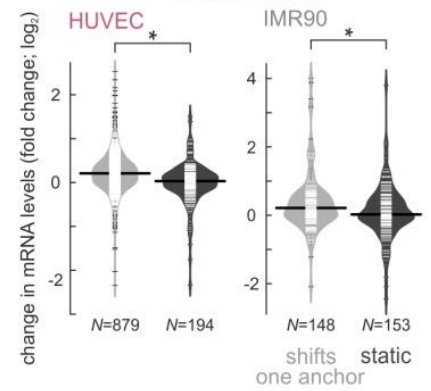


Figure 3.1.S10. Disruption of higher-order organization upon HMGB2 knock-down (related to Figure 3.1.7). (A) Hi-C heatmaps showing interaction frequencies along the long arm of chromosomes 11 and $\mathrm{X}$ (ideograms) in control ("NTC") and knock-down ("siHMGB2") HUVEC at 100- (left), and zoom-ins at 40-kbp resolution (right). Low-resolution maps are aligned to A/B compartment plots. Zoom-in maps are aligned to HMGB2 and CTCF ChIP-seq data, and two regions with changing interactions around HMGB2 peaks are indicated (arrows).

(B) Left column: Heatmaps showing normalized Hi-C interaction frequencies (at 20-kbp resolution) in the $0.4 \mathrm{Mbp}$ around HMGB2, CTCF, and Rad21 peaks in proliferating or NTC HUVEC positioned at TAD boundaries. Right column: Heatmaps showing changes in contact frequency in the same $0.4 \mathrm{Mbp}$ upon senescence entry or HMGB2 knock-down. Rad21/HMGB2 heatmaps refer to data from IMR90 (grey square). The number of peaks $(N)$ used in each heatmap is indicated.

(C) As in panel B, but for all HMGB2, CTCF or Rad21 ChIP-seq peaks.

(D) Table showing (left to right) the total number of read pairs generated for each HiChIP library, the per cent of reads mapped, the number of interaction pairs and read pairs that map to them, and the final number of CTCFCTCF loops called in cis.

(E) Bar plots showing the percentage of all CTCF-CTCF loops detected by HiChIP that do not change ("static"), change only on one end ("one anchor static") or are only seen in proliferating or senescent HUVEC and IMR90 ("unique"). The number of loops detected per cell type and condition $(N)$, and their median length (blue) are indicated below the graph.

(F) CTCF-CTCF loops detected using HiChIP along representative region of different chromosomes. CTCF (black) and HMGB2 peak positions from proliferating (grey) and senescent HUVEC/IMR90 (green) are shown aligned to Refseq gene models, and CTCF loops (colour-coded for intensity; PETs).

(G) Bean plots showing changes in mRNA levels of genes ( $\log _{2}$ fold-change) with 10-kbp of CTCF loops that do not change ("static") or change only on one end ("one anchor static"). The number of genes associated with each CTCF loop subset $(N)$ is indicated. *: significantly different; $P<0.01$, Wilcoxon-Mann-Whitney test. 


\section{Supplemental Tables}

Table 3.1.S4 (related to Figures 3.1.5 and 3.1.7). List of primers used in HMGB2/CTCF ChIP-qPCR experiments.

\begin{tabular}{|c|c|c|c|}
\hline Location (hg19) & Primer pair ID & Forward primer & Reverse primer \\
\hline \multicolumn{4}{|l|}{ HMGB2 primers } \\
\hline $\begin{array}{l}\text { chr10: } 92671441- \\
92671590\end{array}$ & $\# 1$ & ATGCGGGTTTACCATGCAGA & GGCCGAGAGCCATAAAGACA \\
\hline $\begin{array}{l}\text { chr1: } 205091401- \\
205091550\end{array}$ & $\# 2$ & TCAGACTCCGCAGGAAAGGT & AAGTACGCGCCTTGGTGAG \\
\hline $\begin{array}{l}\text { chr1: 205091401- } \\
205091550\end{array}$ & $\# 2$ & TCAGACTCCGCAGGAAAGGT & AAGTACGCGCCTTGGTGAG \\
\hline $\begin{array}{l}\text { chr15: 39890983- } \\
39891451\end{array}$ & $\# 4$ & CATACAATAAAGGTGGTGCCAG & TTCAGGAGCTTAATACTGGAGGC \\
\hline $\begin{array}{l}\text { chr20: } 43229526- \\
43229628\end{array}$ & $\# 8$ & GACACGCTCAATAGGCTGAGT & GGGCTCTTATCCTTTCCCGA \\
\hline $\begin{array}{l}\text { chr7: } 135242558- \\
135242707\end{array}$ & $\# 9$ & GCCTTTTGTACGCCTGGGA & ACCTGAGCGAAGCCTGAAC \\
\hline $\begin{array}{l}\text { chr10: } 12085032- \\
12085261\end{array}$ & $\# 11$ & TGAGTGCCATTCACTTAACAGC & GCTGGTAGTGGCTACCTTACG \\
\hline $\begin{array}{l}\text { chr1: } 54304149- \\
54304338\end{array}$ & $\# 12$ & TCTGGCGACCAAACGGTCC & ATTCCTCCTACTTCCTCTCAGGG \\
\hline $\begin{array}{l}\text { chr1: 71546764- } \\
71546943\end{array}$ & $\# 13$ & CCTGACTCGCGCCTACTACT & CTGGACAACAACGTACAAGCTG \\
\hline $\begin{array}{l}\text { chr3: } 197676616- \\
197677150\end{array}$ & \#3 & TCAGTGCGAAGCCGATTTCC & AACATCTTTCGACTCCGCCC \\
\hline $\begin{array}{l}\text { chr3: } 197676616- \\
197677150\end{array}$ & $\# 3$ & TCAGTGCGAAGCCGATTTCC & AACATCTTTCGACTCCGCCC \\
\hline $\begin{array}{l}\text { chr12: } 110841451- \\
110841950\end{array}$ & $\# 5$ & GGTCCATCGGGTCCACAC & CACCTGAAAAGGACCACGAC \\
\hline $\begin{array}{l}\text { chr12: } 110841451- \\
110841950\end{array}$ & $\# 5$ & GGTCCATCGGGTCCACAC & CACCTGAAAAGGACCACGAC \\
\hline $\begin{array}{l}\text { chr14: } 89290652- \\
89291101\end{array}$ & $\# 6$ & CCAGGGGCAGGTGTCTAACT & GCTTGCCTAAACTTGGTTGGA \\
\hline $\begin{array}{l}\text { chr14: 89290652- } \\
89291101\end{array}$ & $\# 6$ & CCAGGGGCAGGTGTCTAACT & GCTTGCCTAAACTTGGTTGGA \\
\hline $\begin{array}{l}\text { chr4: } 145598489- \\
145598788\end{array}$ & $\# 7$ & TCTCTGGAGCCAAGGACAGA & ACAACACCTCGATTTCCCCT \\
\hline $\begin{array}{l}\text { chr17: } 45000353- \\
45000542\end{array}$ & $\# 10$ & ССTTCTCCGATCTCGCAACC & CCTTCCGGTTTCCGGCTTC \\
\hline \multicolumn{4}{|l|}{ CTCF primers } \\
\hline $\begin{array}{l}\text { chr12: 58934310- } \\
58934609\end{array}$ & CTCF_1 & CTGGGGTTGAGGGATGCAAA & AAGTGCAGTAGTGAACCAGCA \\
\hline $\begin{array}{l}\text { chr2: } 231024897- \\
231025196\end{array}$ & CTCF_2 & ATCTTCCTCCСCTTCAAAGCC & CTCCAGAGACGGAAAGGAATTG \\
\hline $\begin{array}{l}\text { chr2: } 113587071- \\
113587195\end{array}$ & IL1B & ACTACCAGTCCTGACTCCCT & GCCACCGAAGACTATCCTCC \\
\hline $\begin{array}{l}\text { chr7: 22770135- } \\
22771841\end{array}$ & IL6 & CAATAACCACCCCTGACC & AGCTGCGCAGAATGAGATG \\
\hline
\end{tabular}




\begin{tabular}{|c|c|c|c|}
\hline $\begin{array}{l}\text { chr4: } 74609611- \\
74609731\end{array}$ & IL8 & GTGTCCTCCACAGAATGTTGG & TCCAGCTATGCTAAAGCGCA \\
\hline $\begin{array}{l}\text { chr12: } 58934310- \\
58934609\end{array}$ & CTCF_1 & CTGGGGTTGAGGGATGCAAA & AAGTGCAGTAGTGAACCAGCA \\
\hline $\begin{array}{l}\text { chr2: } 231024897- \\
231025196\end{array}$ & CTCF_2 & ATCTTCCTCCCCTTCAAAGCC & CTCCAGAGACGGAAAGGAATTG \\
\hline $\begin{array}{l}\text { chr19: } 4139796- \\
4140095\end{array}$ & CTCF_4 & AGAAGATTTCCGGCAGAGGAG & CCTGGTTTCTCAGCTCCCATT \\
\hline $\begin{array}{l}\text { chr1: 203141394- } \\
203141693\end{array}$ & CTCF_5 & GGTAAGCATCCTGGGCACTC & AGGGGGAGACAGAGAGTCAG \\
\hline $\begin{array}{l}\text { chr11: } 73336782- \\
73337081\end{array}$ & CTCF_6 & GGAAGTAATGCCTCTCGCCT & СTCCATCTGGCTCTTCTGCT \\
\hline $\begin{array}{l}\text { chr1: } 23823644- \\
23823893\end{array}$ & CTCF_7 & AACAGTTCGAGTGGTCAGGG & AGTGGTTGTGTTGACCCAGT \\
\hline $\begin{array}{l}\text { chr6: } 136965421- \\
136965570\end{array}$ & HMGB/CTCF_10 & CCTGTAGGACACACCACAAGG & TGCTGTTTTCCCTGATTCCCA \\
\hline $\begin{array}{l}\text { chr10: } 92671441- \\
92671590\end{array}$ & HMGB/CTCF_4 & ATGCGGGTTTACCATGCAGA & GGCCGAGAGCCATAAAGACA \\
\hline $\begin{array}{l}\text { chr19: } 1848761- \\
1848910\end{array}$ & HMGB/CTCF_5 & GGGCCCGCTATTACTCATTTG & CCAGGTTTAAGGCCACCTCC \\
\hline $\begin{array}{l}\text { chr1: } 205091401- \\
205091550\end{array}$ & HMGB/CTCF_6 & TCAGACTCCGCAGGAAAGGT & AAGTACGCGCCTTGGTGAG \\
\hline $\begin{array}{l}\text { chr11: } 66206561- \\
66206710\end{array}$ & HMGB/CTCF_7 & ATCTGCTGCACACGGTGATA & ACAGCATGTGAGGAACAGAGAG \\
\hline $\begin{array}{l}\text { chr2: } 74619201- \\
74619350\end{array}$ & HMGB/CTCF_9 & GGGAAGCCGATTAGGACCAT & TGCAAATAAGTCCTCCAGCCC \\
\hline $\begin{array}{l}\text { chr5: } 142078622- \\
142078921\end{array}$ & HMGB/CTCF_11 & TGGACTGCACTTTCCACTACC & AAGCCTTGCAGACTCCCATC \\
\hline $\begin{array}{l}\text { chr10: } 92671421- \\
92671670\end{array}$ & HMGB/CTCF_14 & ATGCGGGTTTACCATGCAGA & GGCCGAGAGCCATAAAGACA \\
\hline $\begin{array}{l}\text { chr13:27825572- } \\
27826096\end{array}$ & neoCTCF_1 & TCAGAGGTCGTTCATTGGGAC & CAGAACATGGTTTAACCCGCC \\
\hline $\begin{array}{l}\text { chr17:45000194- } \\
45000494\end{array}$ & neoCTCF_2 & AGAACAGCAGATGATAGCGGC & CTCAGGATTCAGTCAGGCGTT \\
\hline $\begin{array}{l}\text { chr14:89290401- } \\
89290745\end{array}$ & neoCTCF_3 & TCAGATGTTGAGATTCGGTGGG & ACAGTACCATCCACCCATCAAG \\
\hline $\begin{array}{l}\text { chr4:107237138- } \\
107237537\end{array}$ & neoCTCF_4 & ATCCGAGCTTTTCACCCTCTAC & CACAAGTCGGGGTCTTTCCT \\
\hline $\begin{array}{l}\text { chr7:99006271- } \\
99006670\end{array}$ & neoCTCF_5 & CTCATGCCTTAGAGATCGCCT & GGAAAACACATACCGGAAGCTC \\
\hline $\begin{array}{l}\text { chr1:87379554- } \\
87380000\end{array}$ & neoCTCF_6 & ACCGAATGTTGCGTCTTTCTC & TACGGTTGTTGTTGGCGACT \\
\hline $\begin{array}{l}\text { chr1:151299135- } \\
151299504\end{array}$ & neoCTCF_7 & ATGGGCATTATGGGAGTCGTC & TCATCCCTGTTGCCTACGTCT \\
\hline
\end{tabular}




\subsection{Chapter II -HMGB1 as a rheostat of chromatin topology and RNA homeostasis on the path to senescence}

This project was conceived and coordinated by Dr. A. Zirkel, Dr. M. Nikolic and Dr. A. Papantonis. The manuscript was written by Dr. A. Papantonis. I was involved in the majority of the experimental procedures and I fully undertook the project after the departure of by Dr. A. Zirkel, Dr. M. Nikolic.

$\underline{\text { My contribution for this manuscript includes the following experimental procedures }}$

- Culturing the IMR90's and HUVECs to replicative senescence and performing the senescence-associated assays.

- Preparing the samples and performing the Western blots, qRT-PCRs and immunofluorescence stainings

- Knock-down of HMGB1 and downstream applications (RT-PCR, Western blots, Immunofluorescence stainings)

- Optimizing and performing the Chip-seq targeting CTCF, HMGB1 and HMGB2

- Co-immunoprecipitation of HMGB1 followed by Western blot or mass-spectrometry

The following figures and tables were summarized and prepared by A.Z, M.N and A.P using data resulted from my experimental procedures:

- Figure 3.2.1C: Characterization of the protein levels of multiple protein in 4 different passages from proliferative to the senescent state

- Figure 3.2: Identification of HMGB1 binding sites and mapping in respect to the Hi-C maps generated by A.Z and M.N

- Figure 3.4A: The interactive proteome of HMGB1 is consisted of multiple RNA binding proteins

- Figure 3.5A-D: Knockdown of HMGB1 is linked with senescence-associated GO terms

- Figure 3.5E-F: HMGB1 is a bona fide RNA binding protein controlling the splicing events in multiple genes associated with senescence

- Figure 3.S3: HMGB1 binding sites are associated with the binding motifs of senescence associated protein factors surrounding genes relevant to senescence

- Figure 3.S5F: Comparison of HMGB1 binding sites and the differential expression analysis of RNAseq shows the impact of HMGB1 on the induction of senescence 


\section{HMGB1 as a rheostat of chromatin topology and RNA homeostasis on the path to senescence}

Konstantinos Sofiadis ${ }^{1,2, \S}$, Milos Nikolic ${ }^{1, \S}$, Anne Zirkel ${ }^{1, \S}$, Yulia Kargapolova ${ }^{1, \#}$, Natasa Josipovic ${ }^{1,2, \#,}$ Antonis Papadakis ${ }^{3}$, Eduardo G. Gusmao ${ }^{1,2}$, Athanasia Mizi ${ }^{1,2}$, Theodore Georgomanolis ${ }^{1}$, Mirjam Koker ${ }^{1,4}$, Roland T. Ullrich ${ }^{1,4}$, Janine Altmüller ${ }^{5}$, Peter Nürnberg ${ }^{5}$, Andreas Beyer ${ }^{3} \&$ Argyris Papantonis ${ }^{1,2, *}$

${ }^{1}$ Center for Molecular Medicine Cologne, University of Cologne, 50931 Cologne, Germany

${ }^{2}$ Institute of Pathology, University Medical Center Göttingen, 37075 Göttingen, Germany

${ }^{3}$ Cluster of Excellence Cologne for Age-associated Disease, University of Cologne, 50931 Cologne, Germany

${ }^{4}$ Clinic I of Internal Medicine and Center for Integrated Oncology, University Hospital Cologne, 50931 Cologne, Germany

${ }^{5}$ Cologne Center for Genomics, University of Cologne, 50931 Cologne, Germany

§,\#These authors contributed equally to this work

*Corresponding author; Tel.: +49 55139 65734, Email: argyris.papantonis@med.uni-goettingen.de

\subsubsection{Abstract}

Spatial organization and gene expression of mammalian chromosomes are maintained and regulated in conjunction with cell cycle progression. This link is perturbed once cells enter senescence. The highly abundant HMGB1 protein, known to associate with bent and looped DNA, is actively depleted from senescent cell nuclei to act as an extracellular proinflammatory stimulus. Despite its physiological importance, we still lack understanding of the positioning and functional roles of HMGB1 on chromatin in vivo. To address this, we mapped HMGB1 binding genome-wide in different primary cells using a tailored protocol. We then integrated ChIP-seq and Hi-C data with a knot theory approach to uncover HMGB1 demarcation at the boundaries of particular topologically-associating domains (TADs). These TADs harbor genes involved in the key proinflammatory leg of the senescent transcriptional program. Moreover, we used sCLIP and siRNA-mediated knockdown to show that HMGB1 is a bona fide RNA- binding protein also affecting splicing choices. Together, our findings highlight a broader than hitherto assumed role for HMGB1 in chromatin homeostasis connected to cell cycle potency, and allow us to postulate a "rheostat" model for HMGB function with implications in cancer biology.

Keywords: 3D chromatin folding; replicative senescence; chromatin loop; RNA binding; splicing; single cell analysis; inflammation; senescence-associated secretory phenotype 


\subsubsection{Introduction}

The high-mobility group box 1 (HMGB1) protein, a member of the highly conserved non-histone DNA binding HMG protein family, was named after its characteristically rapid electrophoretic mobility (Štros, 2010). HMGB1, together with histone H1, are the next most abundant proteins after core histones, with one HMGB1 molecule for every 10 nucleosomes (Thomas and Stott, 2012). Despite its documented abundance in cell nuclei, HMGB1 (also known as an "alarmin") has been predominantly studied in vivo as an extracellular signaling molecule (Lohani and Rajeswari, 2016; Bianchi et al, 2017). HMGB1 is actively secreted by activated monocytes and macrophages to signal tissue damage, and passively released by necrotic and damaged cells. Once received by cells, HMGB1 molecules can be recognized with high affinity by RAGE receptors to potently signal inflammation (Scaffidi et al, 2002; Bonaldi et al, 2003; Orlova et al, 2007). In cells entering senescence, HMGB1 relocalizes from the nucleus to the cytoplasm and is then secreted to stimulate NF-אB activity via Toll-like receptor signaling. This relocalization and secretion controls and contributes to the senescence-associated secretory phenotype (SASP) of mammalian cells, representing a major paracrine contributor both in vitro and in vivo (Salminen et al, 2012; Acosta et al, 2013; Davalos et al, 2013).

Within proliferating cell nuclei, HMGB1 is thought to bind DNA in a nonspecific manner via its two HMGB-box domains, and to bend and contort the double helix - a mode of action that facilitates recruitment of various DNA-binding factors, like p53 (Štros et al, 2004; Štros, 2010; Rowell et al, 2012). HMGB1 has been studied in detail for its contribution to DNA repair (Ito et al, 2015; Mukherjee et al, 2016), V(D)J recombination (Little et al, 2013; Zagelbaum et al, 2016) or chromatin assembly (Bonaldi et al, 2002), but not so much for its transcriptional role (Calogero et al, 2009). Notably, cells lacking HMGB1 also contain reduced numbers of nucleosomes, rendering chromatin more susceptible to DNA damage, spurious transcription, and inflammatory activation (Giavara et al, 2005; El Gazzar et al, 2009; Celona et al, 2011; De Toma et al, 2014).

HMGB1 associates with its cognate DNA sites with characteristically high "on"/“off” rates, and its acidic tail is important for stabilizing binding (Pallier et al, 2003; Ueda et al, 2004; Štros, 2010; Blair et al, 2016). However, it is now understood that HMG-box DNA-binding domains are particularly sensitive to commonly-used fixatives, like formaldehyde; thus, capturing them on chromatin can be challenging (Pallier et al, 2003; Teves et al, 2016). As a result, there exist no genome-wide datasets describing HMGB binding repertoires in primary mammalian cells (see http://chip-atlas.org), and our appreciation of their onchromatin roles remains vague. To address this, we employed here a tailored dual-crosslinking approach previously used to map HMGB2 binding genome-wide (Zirkel et al, 2018). We can now show that HMGB1 binding in proliferating primary endothelial and lung fibroblast cells is far from nonspecific, while also disparate to that by HMGB2. Once integrated with whole-genome chromosome conformation capture (Hi- 
C) data from proliferating and senescent cells, HMGB1-bound positions demarcate the boundaries of a considerable and specific fraction of topologically-associating domains (TADs; Dixon et al, 2012; Nora et al, 2017). This topological contribution is eliminated upon senescence entry, and knockdown experiments show that HMGB1 directly controls the expression of genes central to the senescent transcriptional program. Critically, as HMGB1 was proposed to also constitute a bona fide RNA-binding protein (Castello et al, 2016), we used sCLIP (Kargapolova et al, 2017) to show it does also influence RNA splicing. In summary, we use senescence as a model to comprehensively characterize the multifaceted in nucleo roles of HMGB1 and show how these converge to underpin transition into replicative arrest by linking chromatin homeostasis with cell cycle progression - a link with far-reaching implications for the attenuation of proliferation of cancer cells overexpressing HMGB1.

\subsubsection{Results}

\section{HMGB1 nuclear loss marks senescence entry coinciding with chromatin changes}

To investigate the in nucleo roles of HMGB1 across cellular contexts we used primary human umbilical vein endothelial cells (HUVECs) and fetal lung fibroblasts (IMR90) that are of distinct developmental origins and have disparate gene expression programs. We defined an early-passage proliferative state and a late-passage senescent state by combining $\beta$-galactosidase staining, cell cycle staging by FACS, and MTT proliferation assays (Figure 3.2.1A). Next, we used RNAseq data from proliferating and senescent HUVEC and IMR90 (from two different biological donors/isolates) to look into changing mRNA levels of chromatin-associated factors. Changes that were convergent between the two cell types involved genes encoding lamin B1, various histones, centrosomal proteins, cohesin and condensin complexes, as well as HMG family proteins, most of which were consistently suppressed upon senescence entry, also at the protein level (Figure 3.2.1B,C; Davalos et al, 2013; Shah et al, 2013; Rai et al, 2014; Zirkel et al, 2018). Here, we chose to focus on HMGB1 due to its high nuclear abundance (Thomas and Stott, 2012; Figure 3.2.1D) and key role in SASP induction (Davalos et al, 2013), but mostly due to its still elusive on- chromatin functions, especially as regards spatial chromatin folding.

HMGB1 immunodetection in early- and late-passage cells documented a $>50 \%$ decrease in its total nuclear levels in senescence-entry cell populations of HUVECs or IMR90 (Figure 3.2.1E). However, HMGB1 nuclear depletion was most dramatic in the enlarged nuclei of senescent cells of either cell type, while smaller nuclei remained largely unaffected. FACS-sorting IMR90 based on light scattering allowed enrichment for cell populations with enlarged nuclei (i.e., $~ 70 \%$ of cells had larger than average nuclei, with $>35 \%$ being $>1.5$-fold larger than the average nucleus of a proliferating cell; Figure 3.2.S1A). This showed that, within such heterogeneous populations, enlarged nuclei almost invariably represent senescent cells lacking HMGB1 and concomitantly exhibiting a drop in H3K27me3 levels that mark facultative heterochromatin - effects which would otherwise be masked (Figure 3.2.1C and 3.2.S1B,C). Last, we 
showed that it is those larger cells that secrete HMGB1, but not HMGB2, into their growth medium to contribute to the SASP (Figure 3.2.S1D).
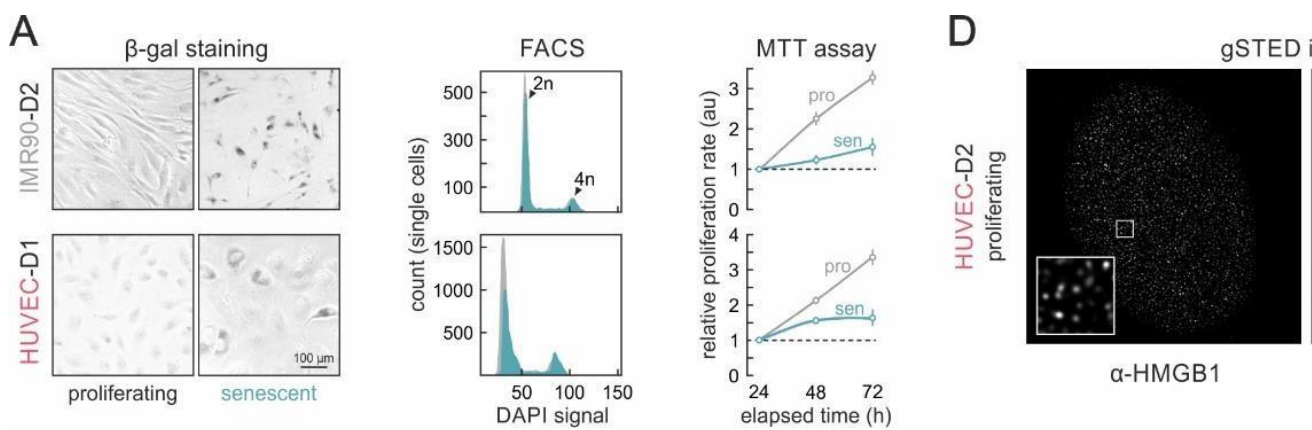

a-HMGB1

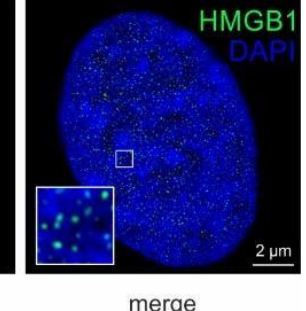

merge

$E$
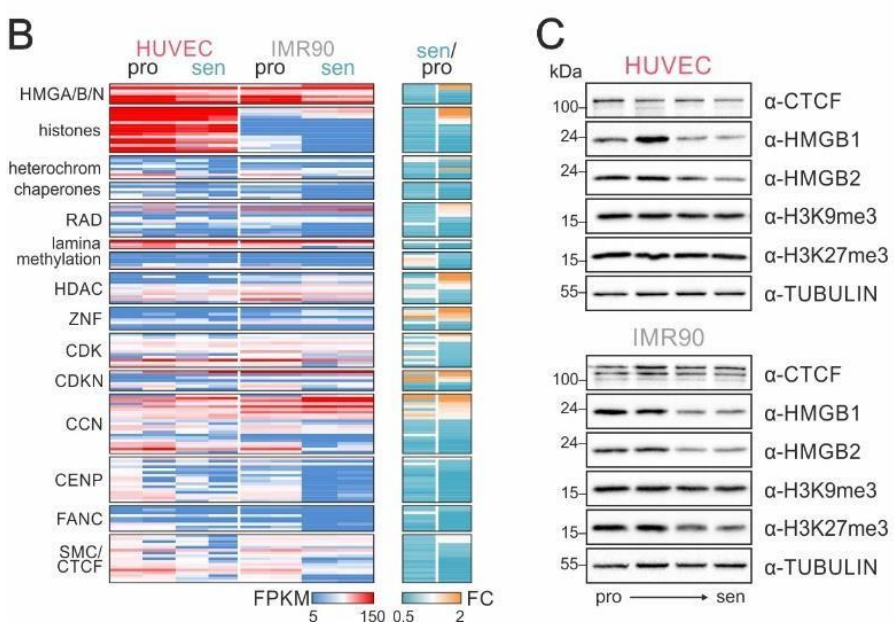

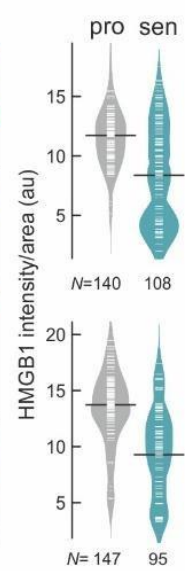

Figure 3.2.1. Senescence entry affects chromatin-binding factors in primary human cells.

(A) Proliferating and senescent IMR90 and HUVECs were tested for $\beta$-galactosidase activity (left), cell cycle progression via FACS (middle), and proliferation rates via MTT assays (right).

(B) Heatmaps show gene expression levels (FPKM; left) and fold-change upon senescence (log2; right) of selected genes encoding chromatin-associated factors.

(C) Western blots show changing protein levels in IMR90 and HUVECs on the path to senescence.

(D) Super-resolution (gSTED) imaging of HMGB1 distribution in proliferating HUVEC nuclei. Bar: $2 \mu \mathrm{m}$.

(E) Representative immunofluorescence images of IMR90 and HUVECs show reduced HMGB1 levels in enlarged senescent nuclei (left), and bean plots quantify this reduction ( $r i g h t ; N$ indicates the number of cells analyzed per each condition/cell type). Bars: $5 \mu \mathrm{m}$.

\section{Regulated HMGB1 nascent transcription in single cells}

Since senescence entry is idiosyncratic to each individual cell and occurs asynchronously in a given cell population, it is important to obtain a single-cell understanding of the transcriptional changes linked to the nuclear loss of HMGB1. To this end, we developed a new protocol for single-cell sequencing of nascent RNA. Typically, scRNA-seq relies on capturing and barcoding cellular mRNAs via reverse- transcription 
of their long poly(A) tails (See et al, 2018). To capture nascent RNA, we decided to add poly(A) tails to any RNA associated with an active site of transcription. We employed the previously- introduced "factory" RNA-seq protocol (Caudron-Herger et al, 2015) as a basis for isolating intact nuclei rich in nascent transcripts from both proliferating and senescent HUVEC. We next removed most chromatin not attached to active transcription sites, and polyadenylated nascent transcripts in situ, before a standard library preparation via a 10X Genomics platform (overview in Figure 3.2.S2A). Using this new approach, and despite low rates of non-duplet capturing (see Methods for more details), we obtained $>550$ single nuclei with an average of 1,650 transcripts in each nucleus, $>55 \%$ of which represented introns or intron-exon junctions. Unsupervised t-SNE clustering of single-nucleus transcriptomes grouped all senescent cells in a single cluster, while subdividing the larger proliferating HUVEC population into three clusters (Figure 3.2.S2B). Reassuringly, genes differentially-expressed amongst proliferating and senescent clusters associated with GO terms relevant to senescence entry and SASP induction (Figure 3.2.S2C).

Mapping nascent RNA levels of individual gene loci onto those clusters, showed that HMGB1 and HMGB2 are actively repressed not only in senescent cells, but also in numerous proliferating cells; the senescence-induced HMGA1/A2 loci strongly produce nascent RNA in senescent cells, but are markedly upregulated also in proliferating cells showing lower HMGB1/B2 transcription (e.g., in cells of the blue cluster; Figure 3.2.S2B). As expected, SASP-related genes like IL4R and MMP14 show strong transcription in cells repressing HMGB1/B2, while the p21-encoding locus, CDKN1A, has most nascent RNA detected in the senescent cluster (Figure 3.2.S2D). Notably, correlating HMGB1 and HMGB2 levels in senescent cells confirms that switching off the latter locus is an early event on the path to senescence (as recently proposed; Zirkel et al, 2018), and this trend is already obvious in proliferating cells (Figure 3.2.S2E). Similarly, correlating HMGB1 levels with those of CDKN1A, HMGBA1 or IL4R, all of which are activated upon senescence entry, we observed that their hyperactivation mostly occurs in those cells where HMGB1 nascent RNA production is diminished (Figure 3.2.S2E).

\section{HMGB1 binds active chromatin loci in a cell type-specific manner}

Capturing HMGB proteins bound to chromatin has proven challenging, because their tandem HMG- box DNA-binding domains are not compatible with standard formaldehyde fixation (Pallier et al, 2003; Belmont et al, 2015; Teves et al, 2016). Here, we employed a tailored fixation strategy in ChIP assays to efficiently capture HMGB1 bound to its cognate sites genome-wide in both proliferating HUVECs and IMR90 (Figures 3.2.2A and 3.2.S3A; see Methods for details). HMGB1 ChIP-seq peaks were predominantly found at the promoters and gene bodies of active genes (>75\% and $>65 \%$ of 810 and 593 peaks in HUVECs and IMR90, respectively; Figure 3.2.2B), overlapping regions marked by H3K27ac and oftentimes H2A.Z (Figure 2.2.2C). HMGB1 peaks were often found clustering along chromosomes of proliferating cells (Figure 3.2.S3B), but as HMGB1 mostly binds active loci, and HUVECs have a gene expression program disparate to that of IMR90, the two repertoires only share 40 peaks (Figure 3.2.S3C). 
It is noteworthy that none of these peaks mark SASP-related genes, despite HMGB1's role in proinflammatory stimulation.

De novo motif discovery in accessible "footprints" within DNase I-hypersensitive sites (derived from ENCODE data) overlapping HMGB1 peaks revealed rather specific CG-rich motifs, which do not however converge into a single consensus sequence (Figure 3.2.S3D). We also surveyed these same accessible footprints for known transcription factor (TF) motifs to infer possible co-binding complexes or complexes that might replace HMGB1 on senescent chromatin. We found that HMGB1 binding sites are significantly enriched for E2F-family motifs, which are notably down-regulated upon senescence entry. Also, motifs for senescence-activated corepressors (e.g., REST and HEY2) and for the architectural ZBTB7B protein that is important for inflammatory gene activation (Nikopoulou et al, 2018) are also enriched therein (Figure 3.2.S3E). Last, we combined RNA-seq with HMGB1 ChIP-seq data to catalogue differentially-expressed genes in either cell type that are directly regulated upon HMGB1 loss in senescence. In both HUVECs and IMR90 we find >2-fold more up- rather than downregulated genes bound by HMGB1 (Figure 3.2.S3F). These senescence-induced genes are involved in ECM organization, regulation of apoptosis, as well as with NOTCH-/TGF $\beta$-signalling that is now understood to represent a second "wave" of paracrine signalling in senescent cells (Hoare et al, 2017). On the other hand, downregulated genes in both cell types converge to cell growth and cell cycle regulation (Figure 3.2.S3F). Together, this data demonstrates how HMGB1 nuclear eviction regulates a critical leg of the program defining senescence entry. 
A

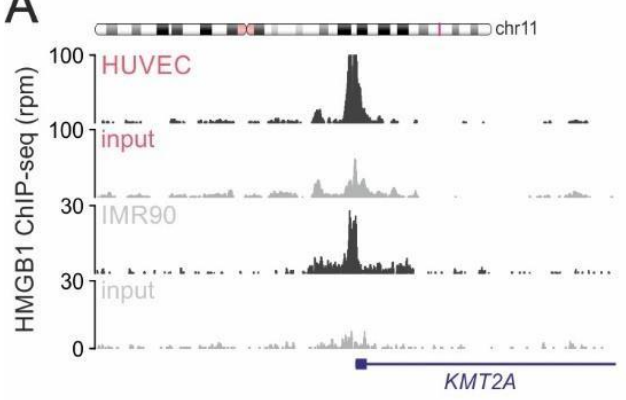

D

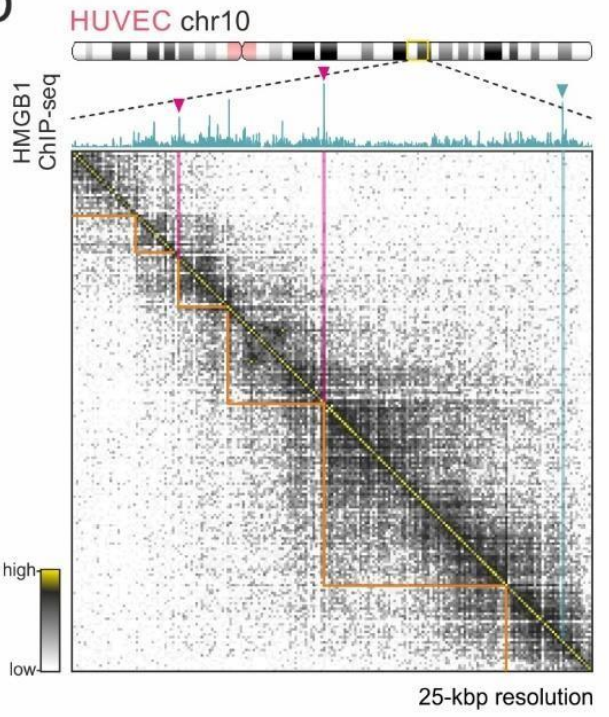

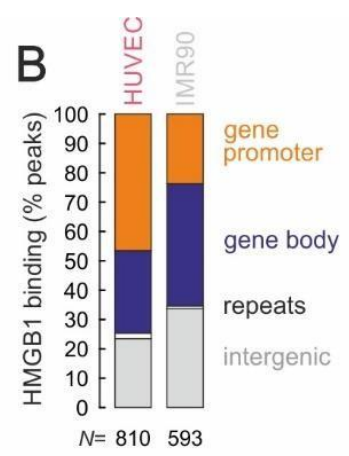

E

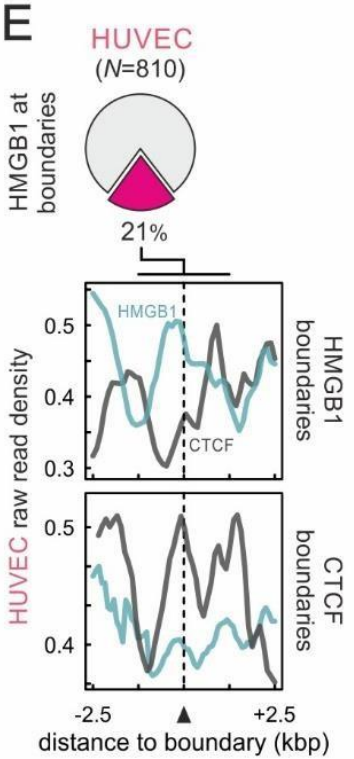

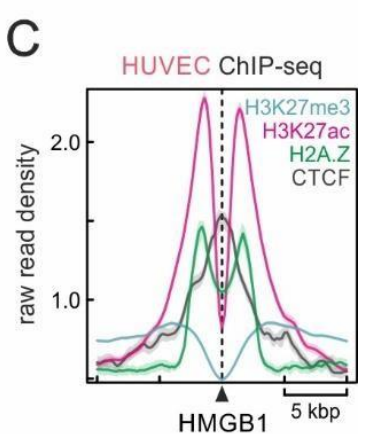
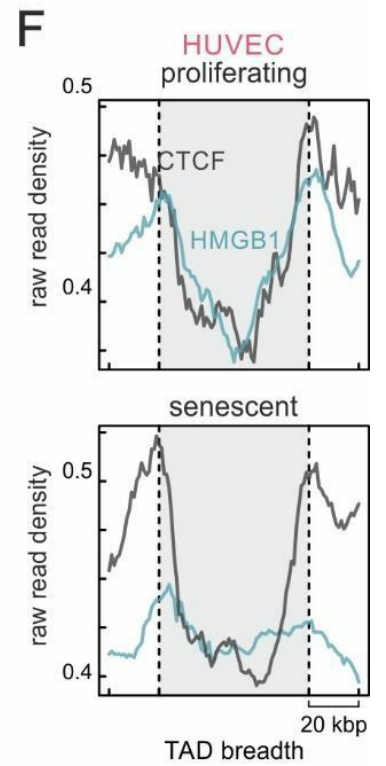

G
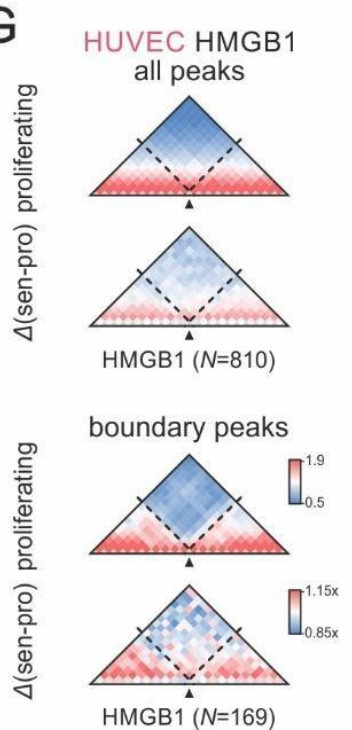

$\mathrm{H}$

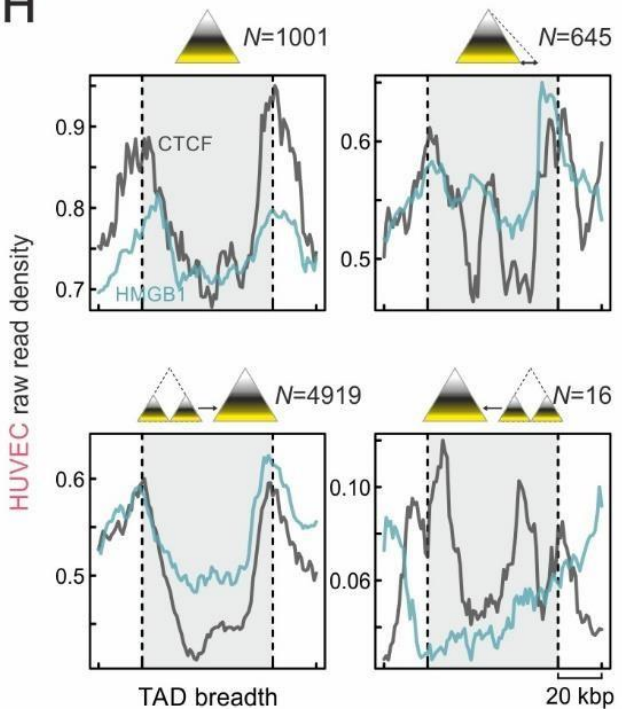

I
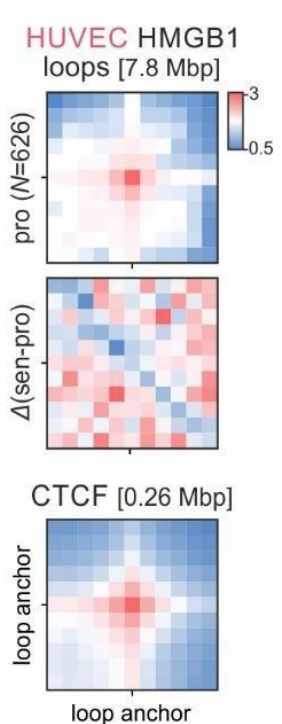

Figure 3.2.2. HMGB1 binds active genes and demarcates a subset of TAD boundaries.

(A) Genome browser views showing HMGB1 ChIP-seq signal at the KMT2A promoter in HUVEC and IMR90 (black); input tracks (grey) provide a negative control.

(B) Bar graphs show the genomic distribution of HMGB1 binding peaks in HUVEC and IMR90. The number of peaks $(N)$ analyzed per each cell type is indicated. 
(C) Line plots show the distribution of H3K27ac (magenta), H3K27me3 (light blue), H2A.Z (green), and CTCF ChIP-seq signal (grey) in the $10 \mathrm{kbp}$ around all HUVEC HMGB1 peaks.

(D) Exemplary Hi-C heatmap for a sub region of HUVEC chromosome 10 aligned to HMGB1 ChIP-seq shows signal peaks (magenta) at TAD boundaries (orange triangles).

(E) Pie chart (top) shows the fraction of HUVEC HMGB1 peaks residing at TAD boundaries. Line plots (below) show the mean distribution of HMGB1 (green) and CTCF ChIP-seq signal (grey) in the $5 \mathrm{kbp}$ around HMGB1or CTCF-marked boundaries.

(F) Line plots show the mean distribution of HMGB1 (green) and CTCF ChIP-seq signal (grey) along all TADs $\pm 20 \mathrm{kbp}$ from proliferating or senescent HUVECs (left) and IMR90 (right).

(G) Heatmaps show mean Hi-C signal from proliferating (top row) or senescent/proliferating HUVEC (bottom row) in the 0.4 Mbp around all HMGB1 peaks (left) or those at TAD boundaries (right). $N$ indicates the number of peaks in each subgroup.

(H) As in panel C, but for TADs that remain unchanged (top left), shift one boundary (top right), merge together (bottom left), or break up upon senescence entry (bottom right). $N$ indicates the number of TADs in each subgroup.

(I) Heatmaps show focal Hi-C interactions between HMGB1-bound peaks from proliferating HUVEC (left) and their loss upon senescence entry (middle); HUVEC CTCF loops provide a positive control (right). Median sizes of CTCF and HMGB1 loops are shown in square brackets.

\section{HMGB1 demarcates a particular subset of senescence-regulated TAD boundaries}

The boundaries of TADs represent genomic sites of strong local insulation of spatial interactions, and are often marked by the presence of CTCF and/or active gene promoters (Dixon et al, 2012; Nora et al, 2017). We recently showed that a considerable number of TAD boundaries in proliferating human cells are marked by HMGB2, and this demarcation is lost upon senescence entry leading to the formation of senescenceinduced CTCF foci (Zirkel et al, 2018). Based on this, we reasoned that HMGB1 might also function similarly Hi-C data from proliferating and senescent HUVEC to investigate this. First, we found that $>20 \%$ of the 810 HMGB1 peaks reside at TAD boundaries (called at 25-kbp resolution; Figure 3.2.2D,E). Remarkably, and much unlike HMGB2, we identified TAD boundaries marked by HMGB1, but lacking CTCF (and the converse; Figure 3.2.2E). As expected, TADs of senescent nuclei lose this demarcation (Figure 3.2.2F), and HMGB1-marked boundaries exhibit an obvious loss of insulation and rearrangement of spatial interactions (Figure 3.2.2G). Next, we grouped TADs according to whether or not their boundaries change upon senescence entry. We found that the $\sim 1,000$ TADs remaining invariant in senescence, boundaries are more enriched for CTCF rather than HMGB1 binding. CTCF and HMGB1 demarcation is comparable in TADs shifting one boundary or collapsing into a larger TAD ( 5,500 TADs in total; Figure 3.2.2H). Thus, we postulate that the loss of HMGB1 in senescence contributes to the remodeling of TADs. Last, we asked whether HMGB1 engages in long-range loop formation; the 810 HMGB1-bound genomic sites give rise to $>600$ interchromosomal loops, which appear to collapse upon senescence entry (Figure 3.2.2I). 


\section{Spatial TAD co-association reveals functional chromosome compartmentalization}

It is now understood that human chromosomes undergo large-scale changes upon replicative arrest (Zirkel et al, 2018), which are accentuated in "deep" senescence (Criscione et al, 2016). Looking at TADs as building blocks of chromosomes (Sexton and Cavalli, 2015), one can ask how these might spatialuly associate to give rise to "meta-TADs" (Fraser et al, 2015) and ultimately to diverse functional topologies. To address this question, we employed a bias-free and unsupervised approach based on "topologicallyintrinsic lexicographic ordering" (TiLO; Johnson, 2014), whereby TADs are treated as nodes in a clustered spatial network tested for robustness via iterative network slicing (see Figure 3.2.3A and Methods for details). By applying this to TADs derived from proliferating and senescent HUVEC Hi-C data, we found that there is a marked increase in clusters that include multiple consecutive TADs in senescence, indicative of TAD merging (Figure 3.2.3B,C). This is consistent with the observation of general spatial chromatin compaction in senescence (Criscione et al, 2016; Zirkel et al, 2018) and with the fact that $~ 75 \%$ of TADs merge in senescence (Figure 3.2.2G). However, we also identified individual ("singular") TADs typically positioned between multi-TAD clusters (Figure 3.2.3C, arrows). Strikingly, the boundaries of singular TADs were strongly marked by HMGB1, compared to the extremities of clusters comprised of $>3$ consecutive TADs (Figure 3.2.3D). To assess the functional impact of this, we investigated expression level changes of genes embedded in different cluster types. Both singular TADs and TADs with $>3$ consecutive TADs harbor genes significantly more upregulated that those in all other TADs, but singular TADs also show significantly more modest gene downregulation (Figure 3.2.3E). Notably, these two subsets harbor most of the genes differentially-regulated upon senescence entry. What further discriminates these clusters functionally, is the fact that multi-TAD clusters uniquely harbor genes involved in the regulation of chromatin organization and conformation, while those unique to singular TADs associate with SASP production and its downstream effects (Figure 3.2.3F). Thus, TiLO identifies functional entities that involve spatial co-association of multiple TADs in cis, explaining the functional specialization of different genomic segments upon HMGB1 loss. 
A

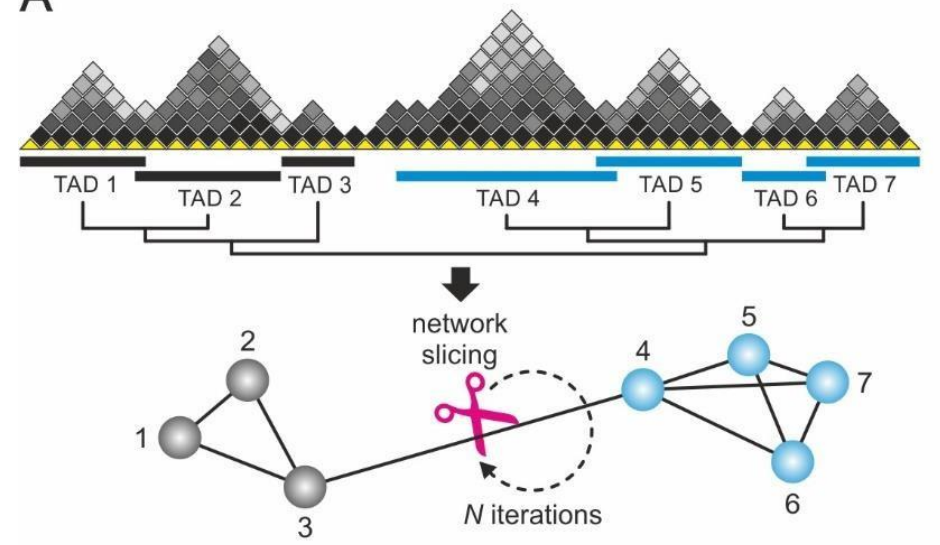

C

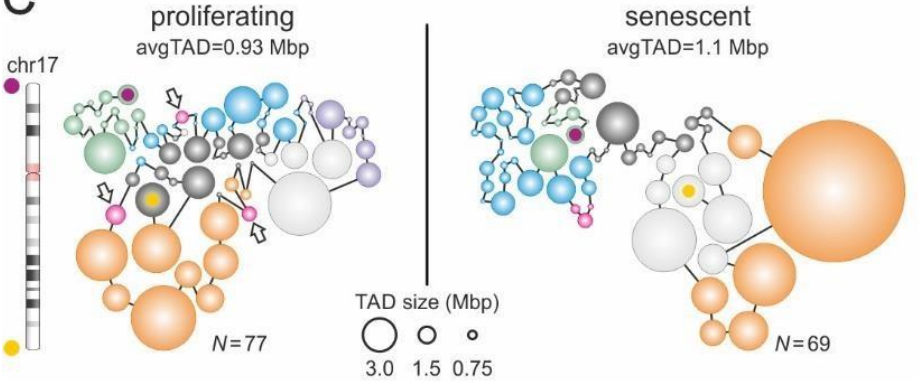

E

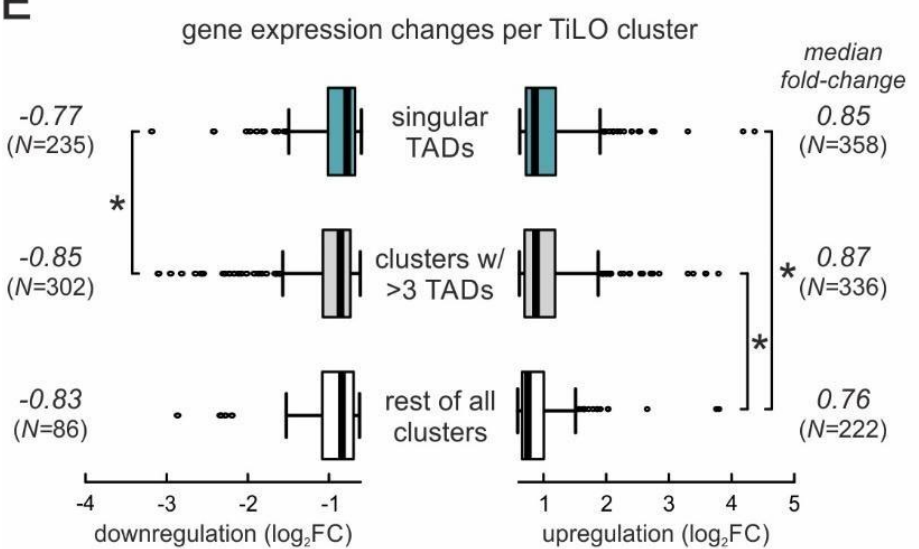

B
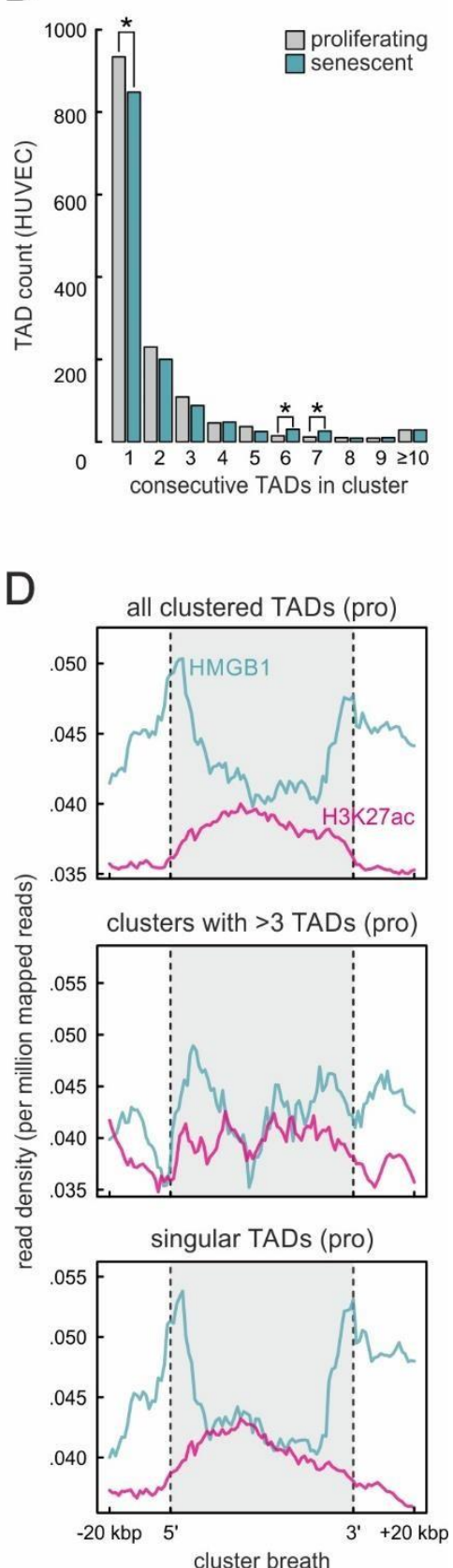

F

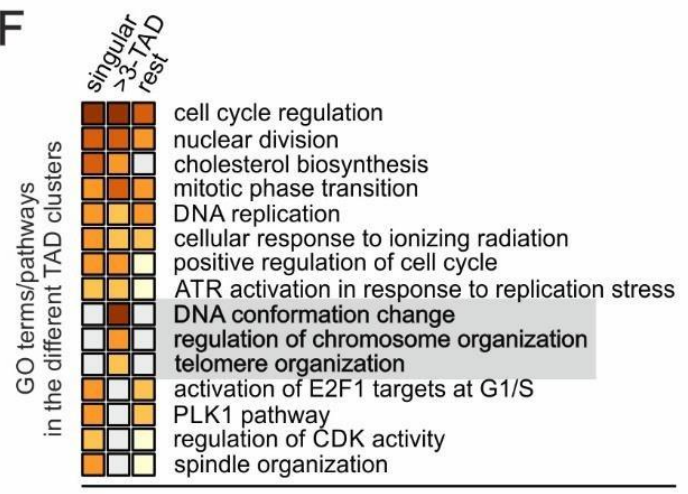

downregulated genes

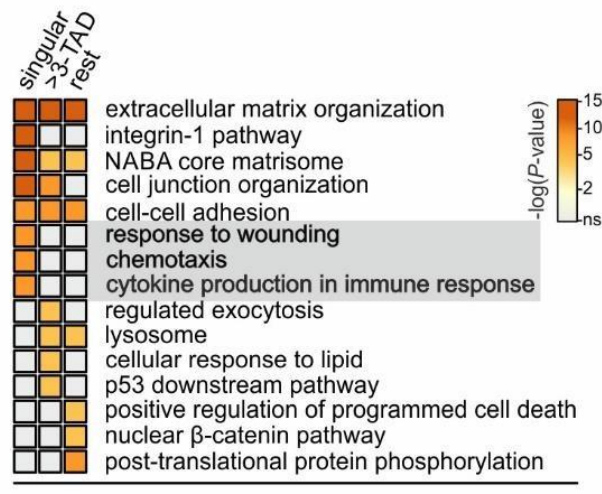

upregulated genes 
Figure 3.2.3 HMGB1 marks individual TADs harboring SASP-related genes.

(A) Overview of TiLO. TADs in each HUVEC chromosome are treated as nodes in an interacting network and interTAD interaction strength is used to infer network connections. These connections are then sliced and network robustness is assessed iteratively; the final network clustering is determined once the minimum number of TAD clusters is reached.

(B) Bar plots show the number of TADs contained in clusters of 1 or more TADs derived using TiLO on proliferating (grey) and senescent HUVEC Hi-C data (green). *: P<0.01; Fisher's exact test.

(C) Illustration of TAD clusters identified using proliferating (left) and senescent HUVEC Hi-C data (right) from chromosome 17. Each sphere represents one TAD; the most 5'/3' TADs are indicated by purple and yellow dots, respectively; arrows indicate "singular" TADs.

(D) Line plots show the mean distribution of HMGB1 (green), CTCF ( rey), and H3K27ac ChIP-seq signal (magenta) at the extremities of all clustered TADs ( $\pm 20 \mathrm{kbp}$; top), of clusters of >3 TADs (middle), or of "singular" TADs (bottom; as those indicated in panel C) from proliferating HUVECs.

(E) Box plots show the $(\log 2)$ fold-change in mRNA levels of senescence-regulated genes embedded in the three cluster categories from panel $D$. The median fold-changes in expression and the numbers of genes in each plot are also indicated. *: $P<0.01$; Wilcoxon-Mann-Whitney test.

(F) Heatmaps show GO terms/pathways associated with differentially-expressed genes in the three cluster categories from panel D. Chromatin organization- and SASP-related GO terms are highlighted (grey rectangles).

\section{HMGB1 directly binds and regulates senescence-relevant transcripts}

On top of its known ability to bind chromatin, HMGB1 was recently also classified as a bona fide RNA- binding protein (Castello et al, 2016; Trendel et al, 2018). Thus, its loss in senescence could also affect some aspect of RNA metabolism. Our suspicion was reinforced by cataloguing the proteins that coimmunoprecipitate with HMGB1 from proliferating IMR90 using mass spectrometry. This returned a diverse range of RNA-binding proteins and splicing regulators, in addition to the expected proteins involved in the regulation of chromatin conformation (Figure 3.2.4A).

To study HMGB1 as a direct RNA binder and regulator, we performed sCLIP (Kargapolova et al, 2017) in proliferating IMR90 in two well correlated biological replicates (Figure 3.2.S4A-C). These two datasets provided a compendium of 1,773 binding peaks on 866 different transcripts (e.g., ASH1L and CCNL2; Figure 3.2.4B and Table S1), which reassuringly display <25\% overlap to HMGB1-bound genes in ChIP-seq. On RNA, HMGB1 was mostly found bound to exons and 5'/3' UTRs, but also to a substantial number of non-coding RNAs (Figure 3.2.4C). These HMGB1 RNA-binding sites present a hexameric 5'NMWGRA-3' $(\mathrm{M}=\mathrm{A} / \mathrm{C}, \mathrm{W}=\mathrm{A} / \mathrm{T}, \mathrm{R}=\mathrm{A} / \mathrm{G})$ motif, irrespective of the predicted folding of the underlying RNA or the exclusion of repeat sequences from the genome build used for motif analysis (Figures 3.2.4D and 3.2.S4D). Much like the trend observed in ChIP-seq data, HMGB1 was found bound to $\sim 3$-fold more transcripts that are up- rather than downregulated upon senescence (Figure 3.2.S4E, left). Upregulated transcripts associated with senescence-relevant GO terms like "ECM organization", "wound healing” and "negative regulation of cell proliferation", while downregulated ones mostly converged on processes like RNA splicing, RNA-/miRNA-mediated gene silencing, or histone remodeling and deacetylation (Figure 3.2.S4E, right). This suggests a feedback loop whereby transcripts encoding RNA regulators are affected by the senescence-induced loss of HMGB1, but also pointed to a more direct impact on splicing regulation. 
We next examined how splicing patterns are altered upon senescence entry by IMR90 using Whippet (Sterne-Weiler et al, 2018). We documented significant changes involving 4,000 transcripts, the majority of which concerned the use of alternative transcription start and polyadenylation sites $(>80 \%$ of cases; Figure 3.2.4E). Interestingly, looking at the splicing changes involving 342 HMGB1-bound mRNAs, this trend remained invariant (Figure 3.2.4E). Finally, we asked whether transcripts undergoing different types of splicing changes are involved in different functional processes. GO term analysis revealed that transcripts with alternative TSS usage encoded regulators of ECM organization and growth control, whereas those with alternative poly(A)-site usage encoded mostly splicing and RNA processing factors; HMGB1-bound transcripts, however, associated with functions from the whole of this spectrum (Figure 3.2.4F). Thus, the nuclear loss of HMGB1 also directly affects processing of the cell's transcriptome.
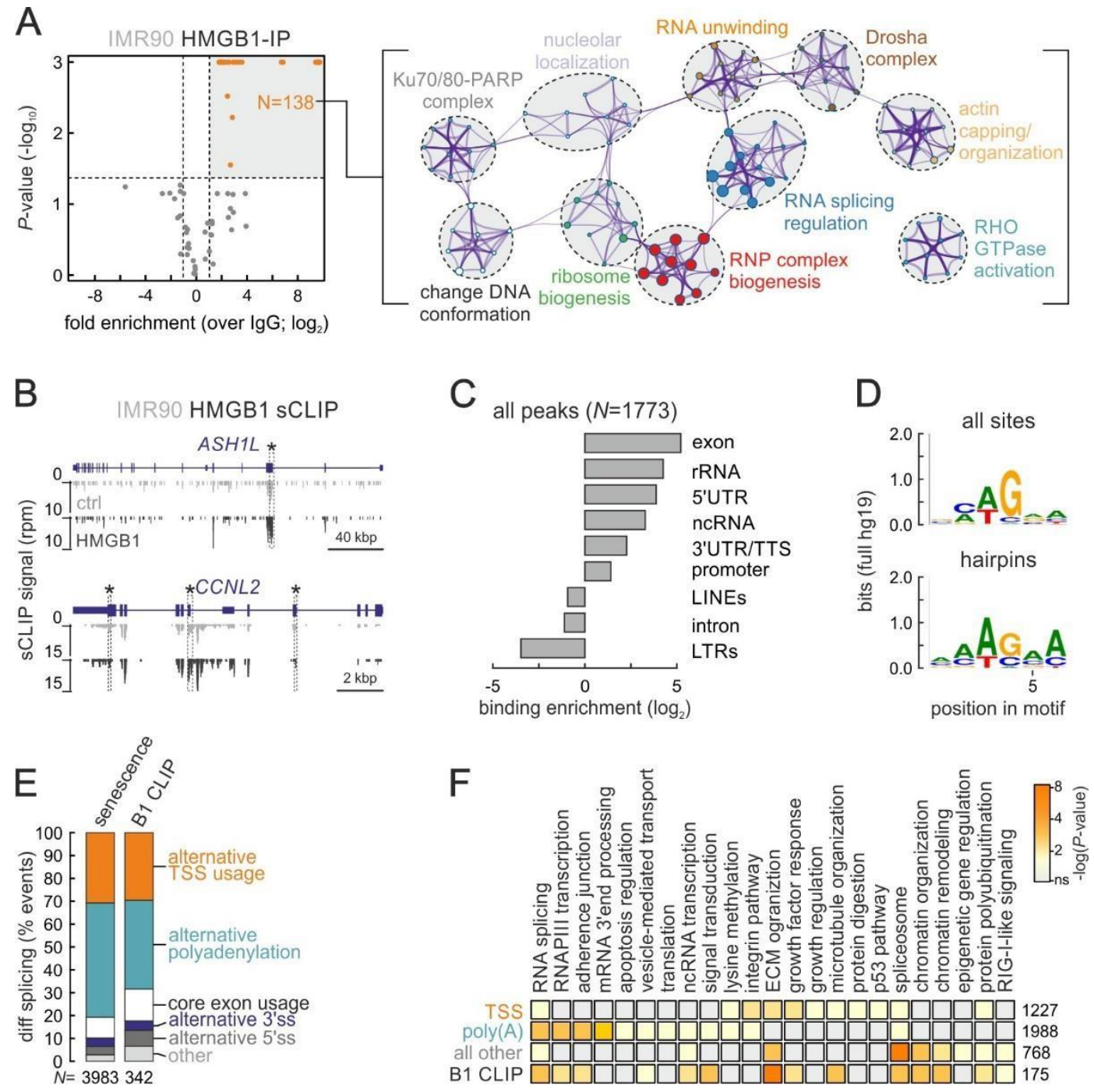
Figure 3.2.4. HMGB1 binds specific mRNAs and affects splicing choices.

(A) Scatter plot (left) shows proteins from proliferating IMR90 co-immunoprecipitating with HMGB1 (orange dots) compared to IgG controls. HMGB1 interactors associate with GO terms/pathways illustrated in the network analysis (right; node size refelcts the number of proteins it includes).

(B) Genome browser views showing HMGB1 sCLIP signal (black) along the ASH1L and CCNL2 IMR90 loci; input tracks (grey) provide a negative control. *: significantly-enriched peak.

(C) Bar graphs show genomic distribution of HMGB1 RNA-bound peaks (log2 enrichment) from proliferating IMR90.

(D) Logos show consensus hexameric motifs deduced from all (top) or hairpin-embedded HMGB1 sCLIP peaks (bottom) using ssHMM analysis.

(E) Bar plots show relative occurrence of differential-splicing events in IMR90 undergoing senescence or only for those mRNAs bound by HMGB1. The number of bound mRNAs $(\mathrm{N})$ analyzed is indicated.

(F) Heatmaps show GO terms/pathways associated with differentially-spliced mRNAs from three categories from panel E (TSS, poly(A), all other) or with all HMGB1-bound mRNAs (B1 CLIP).

\section{HMGB1 depletion underlies induction of the senescence transcriptional program}

It was previously demonstrated that transduction of human fibroblasts with shRNAs against HMGB1 sufficed to induce senescence (Davalos et al, 2013). To avoid using lentiviral vectors, we first treated HUVECs with a pool of self-delivering siRNAs targeting HMGB1. This led to a $\sim 2$-fold reduction of HMGB1 protein and RNA levels within $72 \mathrm{~h}$ (Figure 3.2.S5A), was accompanied by a doubling of $\beta$ gal and p21-positive cells in the knockdown population, but only small changes in nuclear size and BrdU incorporation were recorded (Figure S5B-E). We subsequently turned to IMR90, where standard siRNA transfections allowed for a >10-fold decrease in HMGB1 levels, while also suppressing or inducing expression of known senescence-regulated genes (but only marginally affecting HMGB2; Figure 3.2.5A). "Deep" sequencing and analysis of mRNAs from HMGB1-knockdown and control IMR90 revealed 900 up- and $~ 950$ downregulated genes (Figure 3.2.5B). GO term and gene set enrichment analyses showed that genes that were upregulated associated with the SASP and proinflammatory signalling, whilethose that were downregulated with changes in chromatin organization, transcriptional silencing, and the p53 pathway (Figure 3.2.5C,D).

For a more precise understanding for the role of HMGB1 in these processes, we focused on genes differentially-regulated upon HMGB1-knockdown that also carried at least one HMGB1 ChIP- seq peak. This returned 44 up- and 56 downregulated genes constituting direct HMGB1 targets linked to NF-кB activation, and to chromatin organization or non-inflammatory signalling, respectively (Figure 3.2.S5F). Nonetheless, this left >850 genes in either category that could not be directly linked to an HMGB1 chromatin-binding event. To bridge this gap, we repeated the above analysis using sCLIP binding events. We found 56 and 97 HMGB1-bound mRNAs up- and downregulated upon HMGB1- knockdown, respectively. Consistent to our previous observations, those upregulated could be linked to processes like ECM organization and proinflammatory activation, while downregulated ones to non-inflammatory signalling and the organization of chromatin (Figure 3.2.S5G). Notably, splicing changes deemed significant upon HMGB1-KD displayed an trend in favour of alternative TSS and poly(A)-site usage as, 
essentially identical to that observed in senescence, with $>60 \%$ of senescence splicing events also being recorded in knockdown cells (Figure 3.2.5E). These mRNAs encode factors linked to senescence-regulated processes like cell cycle and cell growth regulation or the p53 pathway (Figure 3.2.5F). Taken together, our data are in support of a model whereby HMGB1 acts both at chromatin loci and on RNA transcripts to regulate cellular functions, and this interplay is disrupted in senescent cells by its nuclear depletion.
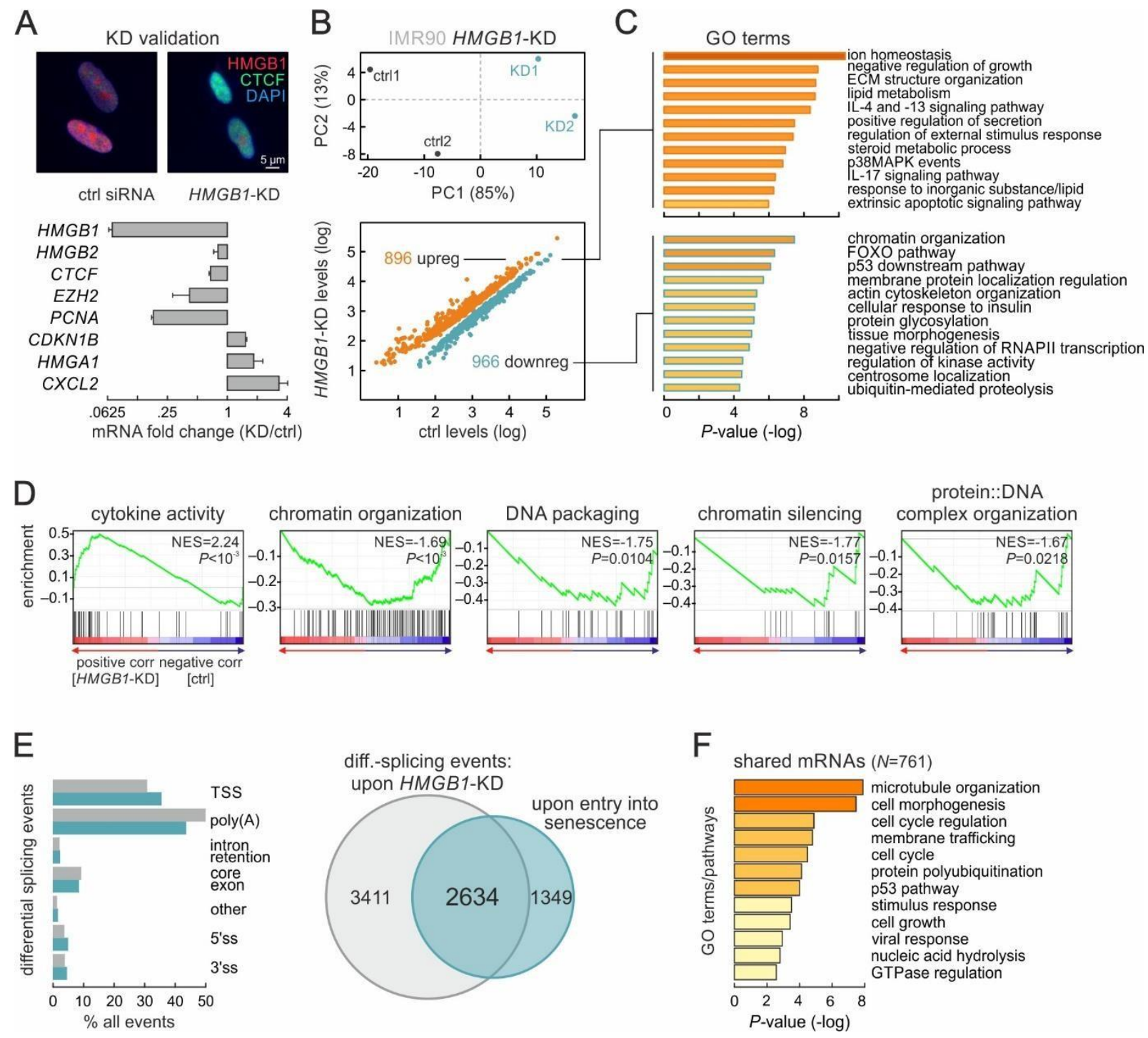

Figure 3.2.5. HMGB1 knockdown induces senescence-specific gene expression changes.

(A) Immunofluorescence (top) and RT-qPCR analyses (bottom; mean fold-change \pm S.D., N=3) confirm HMGB1 knockdown in IMR90 at the protein and mRNA levels, respectively. Bar: $5 \mu \mathrm{m}$.

(B) PCA analysis plot (top) of the two control (black) and HMGB1-knockdown replicates (green). Scatter plot (bottom) shows genes significantly up- $(>0.6 \log 2$-fold change; orange $)$ or downregulated $(<-0.6 \log 2$-fold change; green) upon $H M G B 1$ knockdown.

(C) Bar plots show the most enriched GO terms associated with the gene sets in panel B and their enrichment $P$-values (right).

(D)Gene set enrichment analysis (GSEA) of gene expression changes in HMGB1-KD IMR90. Normalized enrichment scores (NES) and $P$-values are indicated for each correlation. 
(E) Bar plots (left) show occurrence of significant splicing events upon senescence (grey) or HMGB1- KD (green). Venn diagram (right) shows the overlap of these events.

(F) Bar plots show the most enriched GO terms associated with the 761 shared differentially-spliced mRNAs in panel E and their enrichment $P$-values (right)

\subsubsection{Discussion}

Unlike its well documented proinflammatory role, the intracellular positioning and gene expression control exerted by HMGB1 on mammalian chromosomes is poorly understood. Here, we were able to assign a multifaceted role to HMGB1 - as an on-chromatin regulator of active gene loci, and as a bona fide RNA-binding protein recognizing a distinct subset of transcripts. As a result, we can deduce the following. First, we that the loss of HMGB1 from the nuclei of senescent cells mostly triggers upregulation of its previously-bound target loci and mRNAs, suggesting that HMGB1 tends to act a "buffering" factor thereon. Second, that $\sim 1 / 5$ of HMGB1-bound positions mark the boundary of a TAD, and many of these TADs specifically harbor SASP-related genes induced upon senescence entry. Last, that the loss of HMGB1 correlates essentially only with alternative usage of TSS and polyadenylation sites in its bound transcripts. These observations come to substantiate a previous hypothesis that low nuclear titers of HMGB1 are necessary for the fully-fledged development of the SASP (Davalos et al, 2013). This is because there is a need for alleviating the regulatory effects that HMGB1 exerts on active promoters, on mRNAs being processed, as well as on TAD boundaries. This is a rather unique example of a regulatory circuit, where the programmed deregulation in one cellular compartment (the nucleus) is in direct and quantitative crosstalk with the signaling deployed in another (in extracellular space to initiate paracrine activation). Thus, the senescent regulatory program has a strong, if not hierarchical, dependency on the nuclear events preceding SASP deployment.

Recently, we characterized the function of the sister protein to HMGB1, HMGB2, for the entry into replicative senescence (Zirkel et al, 2018). The loss of HMGB2 is an event preceding the loss of HMGB1, and leads to the formation of large senescence-induced CTCF clusters (SICCs). This has an apparent effect on the spatial architecture of chromosomes, and concomitantly on gene expression. Intriguingly, direct HMGB2 target loci are also typically upregulated when relieved of HMGB2 binding; however, this is the only similarity between the functions of HMGB1 and HMGB2. The loss of HMGB1 does not trigger SICC formation, the same way that the loss of HMGB2 does not trigger immediate senescence entry. Also, HMGB1 and HMGB2 bind non-overlapping targets and also demarcate TADs in two distinct modes - HMGB2 marks the extremities of TADs that shift one boundary in senescence, while HMGB1 is mostly found at the boundaries of TADs that collapse together, in line with the overall compaction observed in senescent chromosomes (Criscione et al, 2016; Zirkel et al, 2018). Finally, it is important to note that the HMGB1 knockdown does not reduce HMGB2 levels in primary human cells, nor does the converse hold true, meaning that the two pathways these related factors control do not really cross one another, but are rather deployed in parallel. 
Interestingly, the knockdown of HMGB1 in primary lung fibroblasts exhibits differential gene expression patterns that are partially inversed in the same cells upon senescence entry (e.g., the negative regulation of RNAPII transcription is suppressed in the knockdown, but not in senescence). This hints towards a coordinated counter-regulation of HMGB1 effects by the rest of the program of cells entering senescence. This can be explained by the fact that the nuclear presence of HMGB1 is linked to favorable proautophagic effects that enhance cell survival and limit programmed cell death (Tang et al, 2010). This might also be a simple way to explain the strong overexpression of HMGB1 (and often also HMGB2) in various cancer types ( $\mathrm{Li}$ et al, 2014; Zirkel et al, 2018). This overexpression seems to favor increased cell proliferation ( $\mathrm{Li}$ et al, 2014), and it is only reasonable to assume that its targeting might be an effective anti-cancer strategy. Here, we used three lung adenocarcinoma lines to show that indeed a simple siRNAmediated inhibition of HMGB1 suffices for replicative arrest and cell death. However, we observed that the response of each of these three lines correlated inversely to their proliferation rates, suggesting that higher rates come with stronger addiction to HMGB1 presence. Moreover, the formation (or strengthening) of SICCs in these cancer cells also aligned well with their response to HMGB1 knockdown -i.e., obvious SICC emergence signified replicative arrest.

In summary, the above allows to propose a simple model by which HMGB1 titers can be seen as a "rheostat" of cell cycle potency of a given cell. Primary proliferating cells maintain normal nuclear HMGB1 levels, cells entering senescence arrest upon nuclear depletion of HMGB1, while aberrantly proliferating cancer cells actively overexpress HMGB1 and are addicted to it for propagation (Figure 3.2.6G). Thus, in a next step, elucidating the exact molecular dependencies of cancer cells to HMGB1 (and probably also HMGB2) nuclear overrepresentation for proliferation may lead to new ideas for combinatorial cancer treatments.

\subsubsection{Materials and Methods}

\section{Primary cell culture and senescence markers}

HUVECs from single, apparently healthy, donors (passage 2; Lonza) were continuously passaged to replicative exhaustion in complete Endopan-2 supplemented with 2\% FBS under 5\% CO2. Cells were constantly seeded at $\sim 10,000$ cells $/ \mathrm{cm} 2$, except for late passages when they were seeded at $\sim 20,000$ cells/cm2. Single IMR90 isolates (I90-10 and -79, passage 5; Coriell Biorepository) were continuously passaged to replicative exhaustion in MEM (M4655, Sigma-Aldrich) supplemented with non-essential amino acids and 20\% FBS under 5\% CO2. Senescence-associated $\beta$-galactosidase assay (Cell Signaling) was performed according to the manufacturer's instructions to evaluate the fraction of positively- stained cells at different passages. Cell proliferation was monitored by MTT assays at different passages. In brief, 
$\sim 5,000$ cells are seeded in 96-well format plates in quadruplicates. On the next day, the medium is replaced with $100 \mathrm{ml}$ fresh medium plus $10 \mathrm{ml}$ of a $12 \mathrm{mM}$ MTT stock solution (Invitrogen), and cells are incubated at $37 \mathrm{oC}$ for $4 \mathrm{~h}$. Subsequently, all but $25 \mathrm{~mL}$ of the medium is removed from the wells, and formazan dissolved in $50 \mathrm{~mL}$ DMSO, mixed thoroughly and incubated at $37 \mathrm{oC}$ for $10 \mathrm{~min}$. Samples are then mixed again and absorbance read at $530 \mathrm{~nm}$. Measurements are taken at 24, 48 and $72 \mathrm{~h}$ post-seeding, background subtracted, and normalized to the $24 \mathrm{~h}$ time point. DNA methylation at six selected CpG islands (Franzen et al, 2017) was measured by isolating genomic DNA at different passages and performing targeted pyrosequencing (Cygenia $\mathrm{GmbH})$. Finally, nascent DNA synthesis was monitored by EdU incorporation and subsequent labelling with Click-iT chemistry (Click-iT EdU Imaging Kit; Invitrogen). In brief, cells were incubated in $10 \mathrm{mM}$ EdU for $7 \mathrm{~h}$, fixed using 3.7\% PFA/PBS for $15 \mathrm{~min}$ at room temperature, permeabilized, and labelled as per manufacturer's instructions, before imaging on a widefield Leica microscope.

\section{Immunofluorescence and image analysis}

Proliferating and senescent cells were grown on coverslips from the stage indicated and were fixed in $4 \%$ PFA/PBS for $15 \mathrm{~min}$ at room temperature. After washing once in PBS, cells were permeabilized in $0.5 \%$ Triton-X/PBS for $5 \mathrm{~min}$ at room temperature. Blocking with $1 \% \mathrm{BSA} / \mathrm{PBS}$ for $1 \mathrm{~h}$ was followed by incubation with the following primary antibodies for $1-2 \mathrm{~h}$ at the indicated dilution: mouse monoclonal anti-HMGB1 (1:1000; Abcam ab190377-1F3); rabbit polyclonal anti-HMGB2 (1:1000; Abcam ab67282); mouse monoclonal anti-HMGB1/2 (1:1000; Sigma-Aldrich 12248-3D2); rabbit polyclonal anti-CTCF (1:500; Active motif 61311); rabbit polyclonal anti-H3K27me3 (1:1000; Diagenode C15410069); mouse monoclonal anti-p21 (1:500; Abcam ab184640-GT1032); rabbit polyclonal anti- lamin B1 (1:2000; Abcam ab16048); mouse monoclonal anti- $\beta$-tubulin (1:1000; Sigma-Aldrich T0198- D66). Following immunodetection, cells were washed twice with PBS for $5 \mathrm{~min}$ before incubating with secondary antibodies for $1 \mathrm{~h}$ at room temperature. Nuclei were stained with DAPI (Sigma-Aldrich) for $5 \mathrm{~min}$, washed, and coverslips mounted onto slides in Prolong Gold Antifade (Invitrogen). Note thatfor gSTED microscopy only, the 2C Pack STED 775 secondary antibodies (1:2000; Abberior 2-0032-052-6) were used. For image acquisition, a widefield Leica DMI 6000B with an HCX PL APO 63x/1.40 (Oil) objective was used; confocal and super-resolution images were acquired on a Leica TCS SP8 gSTED microscope with a 100x/1.40 (Oil) STED Orange objective. For immunofluorescence image analysis, the NuclearParticleDetector2D of MiToBo (version 1.4.3; available at http://mitobo.informatik.unihalle.de/index.php/Main_Page) was used. Measurements of nuclear immunofluorescence signal were automatically generated using a mask drawn on DAPI staining to define nuclear bounds. Background subtractions were then implemented to precisely determine the mean intensity per area of each immunodetected protein. Deconvolution of super-resolution images was performed using the default settings of the Huygens software (Scientific Volume Imaging). 


\section{Protein extraction and western blotting}

For assessing protein abundance at different passages, $\sim 4 \times 106$ cells were gently scraped off 15 -cm dishes, and pelleted for $5 \mathrm{~min}$ at $600 \mathrm{x}$ g. The supernatant was discarded, and the pellet resuspended in $100 \mathrm{~mL}$ of ice-cold RIPA lysis buffer (20 mM Tris-HCl pH 7.5, $150 \mathrm{mM} \mathrm{NaCl}, 1$ mM EDTA pH 8.0, 1 mM EGTA $\mathrm{pH}$ 8.0, 1\% NP-40, $1 \%$ sodium deoxycholate) containing 1x protease inhibitor cocktail (Roche), incubated for $20 \mathrm{~min}$ on ice, and centrifuged for $15 \mathrm{~min}$ at $>20,000 \mathrm{x}$ g to pellet cell debris and collect the supernatant. The concentration of the nuclear extracts was determined using the Pierce BCA Protein Assay Kit (Thermo Fisher Scientific), before extracts were aliquoted and stored at $-70 \mathrm{oC}$ to be used for western blotting. Resolved proteins were detected using the antisera mentioned above, plus a mouse monoclonal antiH3K9me3 (1:200; Active motif 39286).

\section{Chromatin immunoprecipitation (ChIP) sequencing and analysis}

For each batch of ChIP experiments $~ 25$ million proliferating cells, cultured to $>80 \%$ confluence in 15 cm dishes, were crosslinked in 15 mM EGS/PBS (ethylene-glycol-bis-succinimidyl-succinate; Thermo) for $20 \mathrm{~min}$ at room temperature, followed by fixation for $40 \mathrm{~min}$ at $4 \mathrm{oC}$ in $1 \%$ PFA. From this point onward, cells were processed via the ChIP-IT High Sensitivity kit (Active motif) as per manufacturer's instructions. In brief, chromatin was sheared to 200-500 bp fragments on a Bioruptor Plus (Diagenode; 2x 9 cycles of $30 \mathrm{sec}$ on and $30 \mathrm{sec}$ off at the highest power setting), and immunoprecipitation was carried out by adding $4 \mathrm{mg}$ of a monoclonal HMGB1 antiserum (Developmental Studies Hybridoma Bank; PCRP-HMGB1$4 \mathrm{~F} 10$ ) to $\sim 30 \mathrm{mg}$ of chromatin and rotating overnight at $4 \mathrm{oC}$ in the presence of protease inhibitors. Following addition of protein A/G agarose beads and washing, DNA was purified using the ChIP DNA Clean \& Concentrator kit (Zymo Research) and used in next-generation sequencing on a HiSeq4000 platform (Illumina) to obtain at least 25 million reads were obtained of both sample and its respective ' 'input'. Raw reads (typically 100 bp-long) were mapped to the reference human genome (hg19) using BWA (Li and Durbin, 2010), and the resulting .BAM files were processed using Picard tools (http://broadinstitute.github.io/picard/) before MACS2 software (Zhang et al, 2008) was used to identify signal enrichment over input. Thresholded HMGB1 ChIP-seq peaks per each cell type were annotated using Chipseeker (Yu et al, 2015) and are listed in Table S2A; .BAM files were used in ngs.plot (Shen et al, 2014) for plotting signal coverage over particular genomic positions for different conditions/cell types. Finally, transcription factor recognition motif enrichments within DHS footprints under HMGB1 ChIP-seq peaks were calculated using the Regulatory Genomics Toolbox (Gusmao et al, 2014). Note that all other ChIP-seq datasets used here come from previous work (Zirkel et al, 2018). 


\section{Whole-genome chromosome conformation capture (Hi-C) and TiLO analysis}

Hi-C data from proliferating and senescent HUVEC were generated previously (Zirkel et al, 2018), and the HiTC Bioconductor package was used to annotate, correct data for biases in genomic features (Servant et al, 2012), and visualize 2D heatmaps with a maximum resolution of 20-kbp. For plotting insulation heatmaps and "loop-o-grams", normalized interactions values in the twenty 20-kbp bins around each HMGB1 peak were added up, normalized to the median value in each matrix and plotted provided the local maxima are higher than the third quantile of Hi-C data in the matrix. All R scripts are available on request; HMGB1-anchored loops are listed in Table S2B.

For Topologically-intrinsic Lexicographic Ordering (TiLO), we directly applied an algorithm from mathematical knot theory that makes zero assumptions about the structure, shape or number of clusters in the data (Johnson, 2014). In brief, topologically-intrinsic ordering was used to permutate the linear order of TADs (as a starting organization level in the Hi-C matrices) until a certain "robustly irreducible" topological condition is satisfied. Then, the "pinch ratio" algorithm is used (Heisterkamp and Johnson, 2013) is applied to heuristically slice the network at connections between TADs were local interaction minima are, while also considering noise in the matrices. Finally, this analysis returns a list of TADs grouped into multiple clusters in cis, also via its built-in measure for network robustness defining the endpoint.

\section{Single-cell nascent RNA sequencing and analysis}

Proliferating (p. 4) and senescent HUVEC (p. 16) were washed once in an isotonic near-physiological buffer (PB) that maintains the cells' transcriptional activity and subjected immediately to the first steps of the "factory RNA-seq" protocol (Melnik et al, 2016). In more detail, cell nuclei are gently isolated using $\mathrm{PB}+0.4 \%$ NP-40, DNase I-treated at $33 \mathrm{oC}$ for $25 \mathrm{~min}$ to detach most chromatin, pelleted and washed once in ice-cold PB, before polyadenylation of nascent RNA as described (Kargapolova et al, 2017). Next, $\sim 2,500$ cells from each state were loaded onto the Chromium 10X Genomics platform for encapsulation in oil droplets and generation of barcoded cDNA libraries from individual nuclei as per manufacturer's instructions. Despite the documented $0.8 \%$ chance of capturing a cell duplet on this platform, HUVEC nuclei are particularly prone to aggregation. As a result, 494 proliferating and 129 senescent single nuclei were efficiently captured and processed. Following sequencing on a HiSeq4000 platform (Illumina), and mapping to the reference genome (hg38) using STAR (Dobin et al, 2013) and filtering via UMI-tools (Smith et al, 2017), $\sim 45,000$ and $\sim 60,000$ reads were generated per each proliferating or senescent cell, respectively. Poor quality cells were excluded (i.e. cells with $<300$ or $>5,000$ expressed genes), as were genes expressed $<10$ cells. This returned 1,650 robustly captured transcripts per cell on average, with $>55 \%$ of reads mapping to introns or exon-intron junctions, 575 genes being expressed in at least $25 \%$ of all cells, and with the 50 most-expressed genes taking over $22 \%$ of all sequencing reads. Mapped and filtered data 
were then processed and visualized using a compilation of ZINB-WaVE (ver. 1.3.4; Risso et al, 2018) and Seurat (ver. 2.3.4; Butler et al, 2018) for clustering and t-SNE visualization. ZINB-WaVE was used to create a low-dimensional representation of the gene expression matrix, and factors inferred in the ZINBWaVE model were added as one of the low-dimensional data representations in the Seurat object in order to identify cell subpopulations via a shared nearest neighbour (SNN) modularity optimization-based clustering algorithm as applied in the FindClusters function of Seurat. Visualization was performed using the t-SNE map by applying the Rtsne function on the ZINB-WaVE output. This map was integrated into the Seurat object and used to plot gene expression. For differential gene expression analysis between the clusters, we applied a combination of ZINB-WaVE and DESeq2 (Love et al, 2014), where the posterior probabilities that counts were generated from the negative binomial count component of the ZINB-WaVE model were used as observation-level weights in the estimation of regression parameters in DESeq2 (Van den Berge et al, 2018). Differentially-expressed genes identified via this method were filtered using a threshold of $\log 2 \mathrm{FC}> \pm 1$ plus $\mathrm{P}$-value $<0.05$ and are listed in Table S3A.

\section{HMGB1 sCLIP and analysis}

sCLIP was performed on $\sim 25$ million UV-crosslinked nuclei from proliferating IMR90 as previously described (Kargapolova et al, 2017) using the same the monoclonal HMGB1 antiserum (DSHB; PCRPHMGB1-4F10) as for ChIP. Following sequencing of strand-specific libraries on a HiSeq4000 platform (Illumina), raw reads were mapped to the human reference genome (hg19). Consistent peaks were identified by overlapping intervals of peaks with a P-value $<0.05$ from 2 biological replicates to obtain 1773 peaks. This peak annotation was used to count reads uniquely aligned to each peak region using HTSeq, HMGB1bound transcript coordinates were retrieved via Ensembl (GRCh37, ver. 78) and annotated using HOMER (http://homer.ucsd.edu), while Gene Ontology analysis was performed using Metascape (www.metascape.org). Finally, the final merged peak list was use for de novo motif analysis using ssHMM (Heller et al, 2017) and significantly enriched motifs were next compared to existing RBP motifs to predict proteins potentially recognizing similar sequences using Tomtom (http://meme- suite.org/tools/tomtom). All HMGB1-bound mRNAs are listed in Table S1.

\section{siRNA-mediated HMGB1 knockdown}

HUVECs were seeded at $\sim 20,000$ cells/cm2 the day before transfection. Self-delivering Accell-siRNA pools (Dharmacon) targeting HMGB1, plus a non-targeting control (NTC; fluorescently-tagged to allow transfection efficiency to be monitored), were added to the cells at a final concentration of $1 \mathrm{mM}$. Knockdown efficiency was assessed $72 \mathrm{~h}$ after transfection using RT-qPCR and immunofluorescence. For the knockdown in lung adenocarcinoma lines and IMR90 cells, standard siRNA transfections were carried out using RNAiMAX (Invitrogen) as previously described (Zirkel et al, 2018). 


\section{Total RNA isolation, sequencing, and analysis}

Control and HMGB1-knockdown were harvested in Trizol LS (Life Technologies) and total RNA was isolated and DNase I-treated using the DirectZol RNA miniprep kit (Zymo Research). Following selection on poly(dT) beads, barcoded cDNA libraries were generated using the TruSeq RNA library kit (Illumina) and were paired-end sequenced to at least 50 million read pairs on a HiSeq4000 platform (Illumina). Raw reads were mapped to the human reference genome (hg19) using default settings of the STAR aligner (Dobin et al, 2013), followed by quantification of unique counts using featureCounts (Liao et al, 2014). Counts were further normalized via the "top quantile" function of RUVseq (Risso et al, 2014) to correct for unwanted variation, before differential gene expression was estimated using DESeq2 (Love et al, 2014). Genes with an FDR $<0.01$ and an absolute ( $\log 2)$ fold-change of $>0.6$ were selected as differentiallyexpressed and are listed in Table S3B. For splicing analysis, a reference index on the basis of hg19 annotation was first constructed, combined with all splice sites contained in the mapped RNA-seq reads. Raw reads were then aligned using Whippet (Sterne-Weiler et al, 2018) to the constructed index in order to quantify and annotate alternative splicing events. Subsequent box/bean plots were plotted using BoxPlotR (http://shiny.chemgrid.org/boxplotr/), and GO term/pathway enrichment plots using Metascape (http://metascape.org/gp/index.html; Tripathi et al, 2015).

\section{Statistical tests}

P-values associated with Student's t-tests and Fischer's exact tests were calculated using GraphPad (https://graphpad.com/), and those associated with the Wilcoxon-Mann-Whitney test using the $\mathrm{U}$ test interface (https://www.socscistatistics.com/tests/mannwhitney/Default2.aspx). Unless otherwise stated, Pvalues $<0.01$ were deemed as statistically significant. 

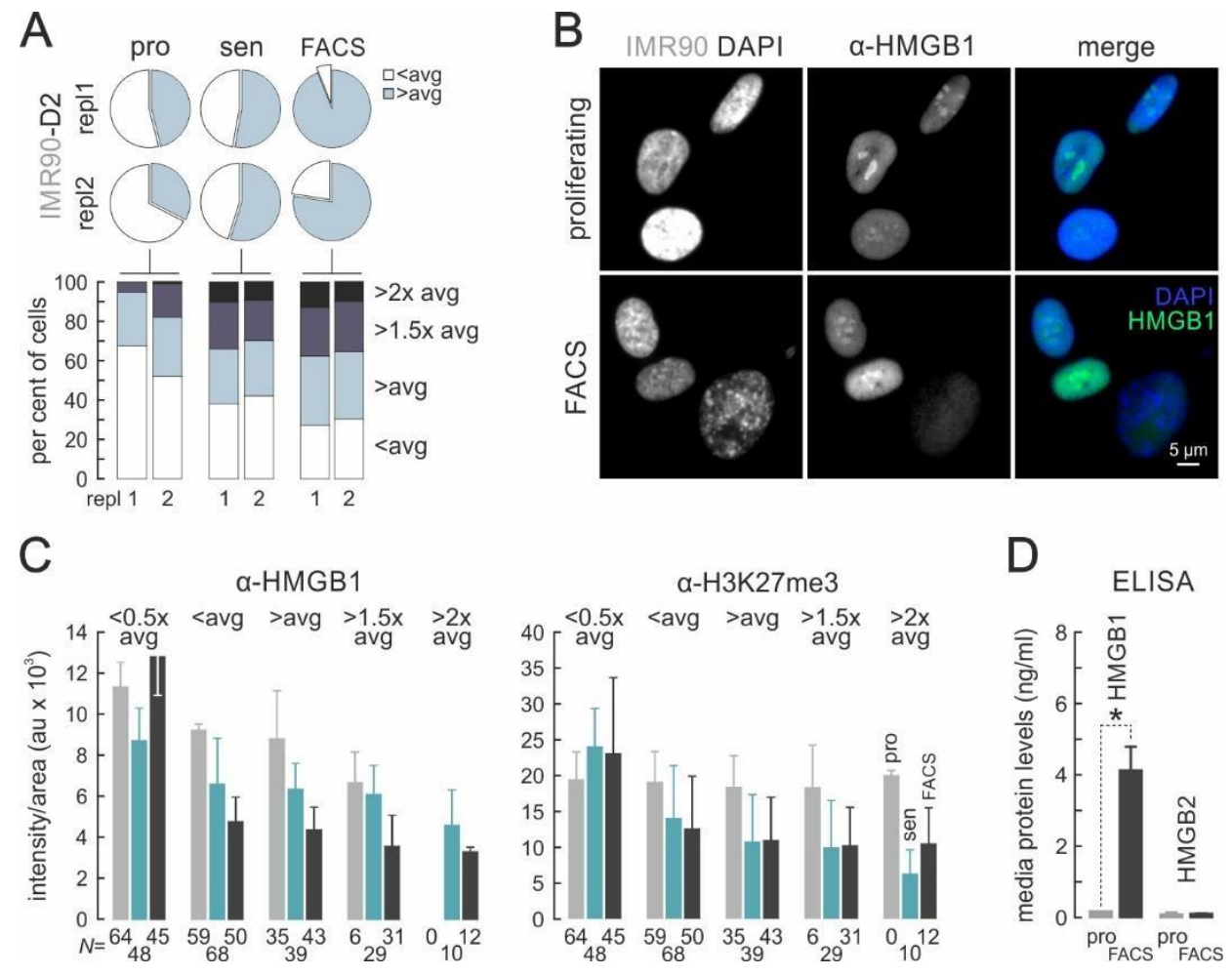

Figure 3.2.S1. HMGB1 depletion mostly occurs in enlarged nuclei.

(A) Pie charts (top) show senescent IMR90 cell populations containing consistently larger nuclei, which can be enriched via FACS sorting. Bar graphs (bottom) show stratification of population by increasing nuclear size (compared to the population average).

(B) Representative immunofluorescence images of proliferating (top row) and senescent FACS-sorted IMR90 (bottom row) show reduced HMGB1 levels in enlarged nuclei. Bar: $5 \mu \mathrm{m}$.

(C) Bar graphs show reduced mean HMGB1 (left) and H3K27me3 levels \pm S.D. (right) in proliferating (grey), senescent (green), and FACS-sorted IMR90 (black) stratified according to nuclear size from images like those in panel B. $N$ indicates the number of cells analyzed in each subgroup.

(D) Bar graphs show mean HMGB1/B2 protein levels \pm S.D. in the growth medium of proliferating (grey) or senescent IMR90 (green). *: $P<0.01$; unpaired two-tailed Student's t-test $(N=2)$. 
A

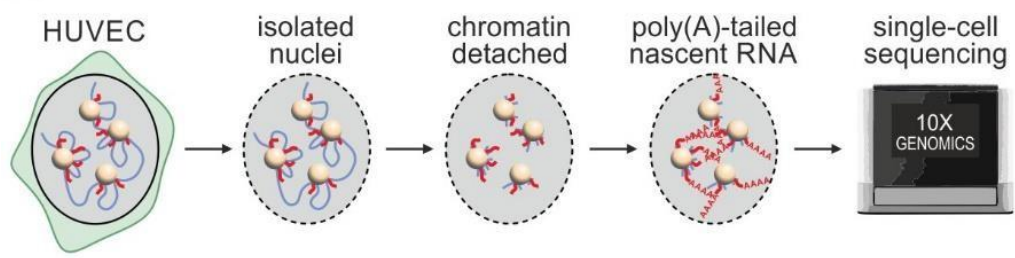

B

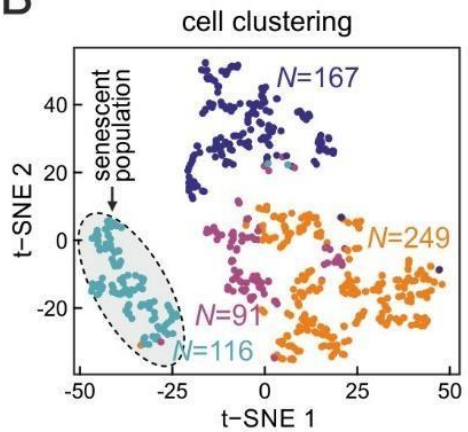

\section{C}

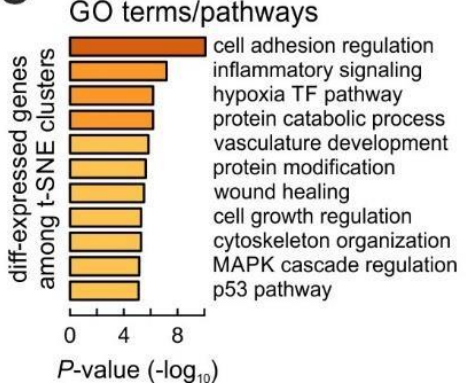

D

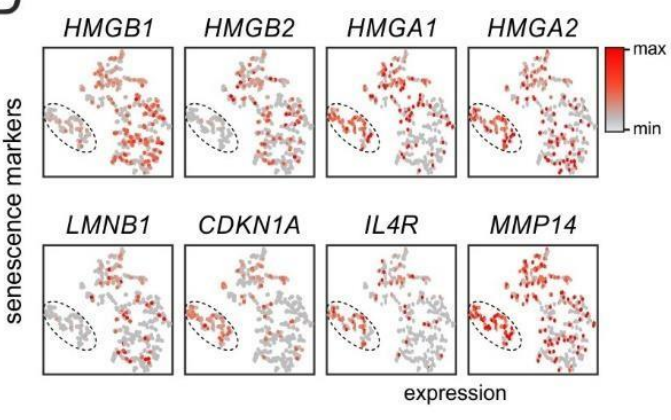

E

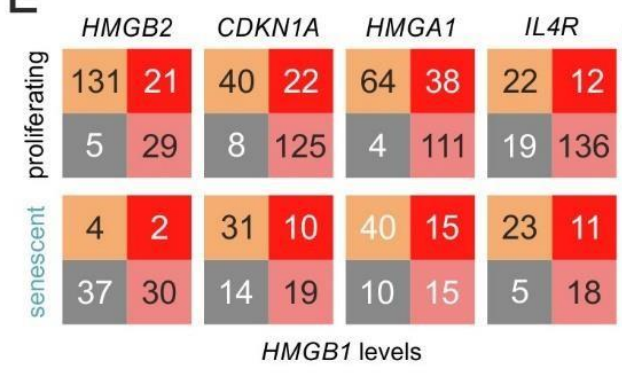

Figure 3.2.S2. Single-cell sequencing of nascent RNA.

(A) Strategy for nascent scRNA-seq involves isolation of intact HUVEC nuclei, detachment of non- transcribed chromatin (blue) via DNase I digestion, and in situ polyadenylation of nascent RNA (red) at transcription sites (spheres), before processing on a 10X Genomics platform.

(B) t-SNE clustering of proliferating and senescent cell populations (grey oval) revealed 4 distinct clusters (blue, red, purple, and green; $N$ indicates the number of cells per cluster).

(C) Bar graphs show (-log) enrichment $P$-values for GO terms associated with differentially-expressed genes amongst clusters in panel B.

(D) Heatmaps show normalized expression of exemplary senescence marker genes in single cells.

(E) Correlation of $H M G B 1$ expression to that of genes from panel D in single proliferating (top row) or senescent HUVEC (bottom row; low: $\leq 1$, high: $>1$ transcript per million). 
A

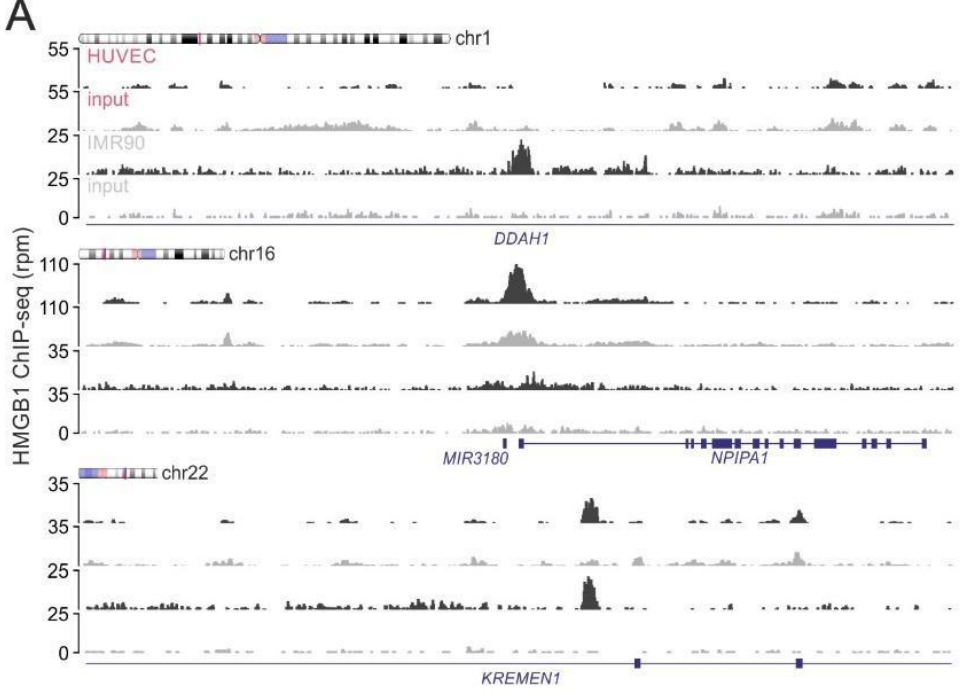

C

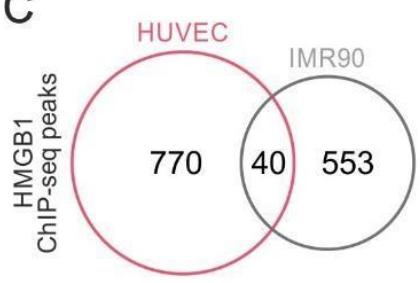

$\mathrm{D}$

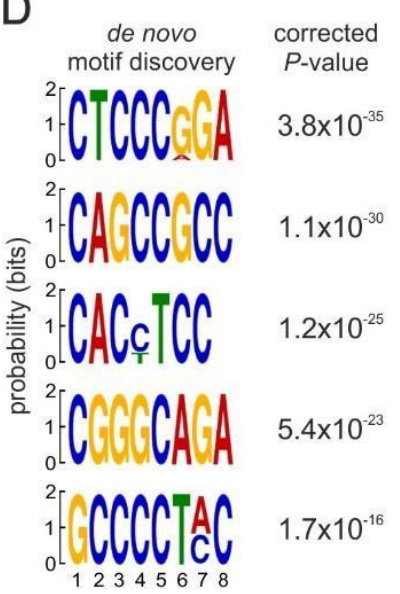

$\mathrm{E}$

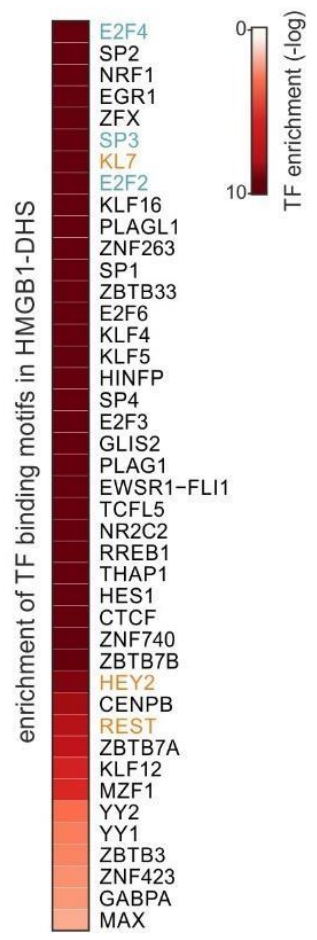

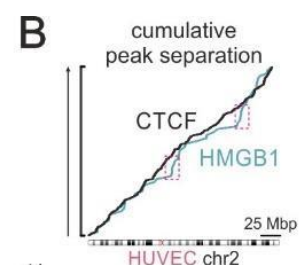
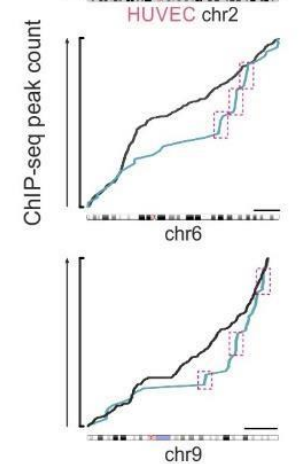

$\mathrm{F}$
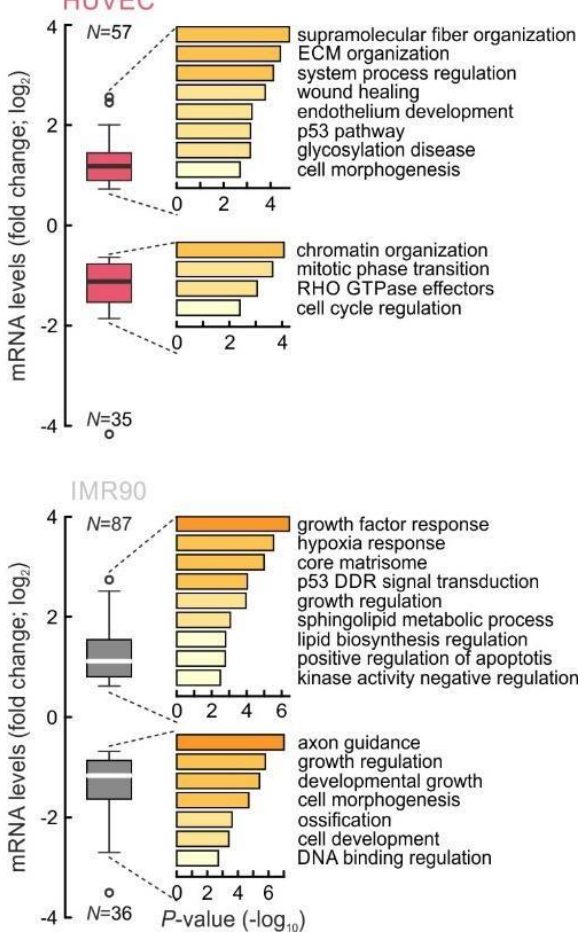

Figure 3.2.S3. HMGB1 Chromatin-binding features in proliferating cells

(A) Genome browser views showing HMGB1 ChIP-seq signal from loci in chromosomes 1, 16, and 22 of both HUVEC and IMR90 (black); input tracks (grey) provide a control.

(B) Plots show the cumulative distribution of HMGB1 binding peaks along HUVEC chromosomes 2, 6 and 9 (green); the distributions of CTCF peaks (black) provide a reference.

(C) Venn diagrams show minimal overlap between HMGB1 binding peaks from HUVEC and IMR90. (D)Logos for the five most enriched de novo motifs discovered under accessible HMGB1 peaks.

(D) Heatmap shows most enriched TF binding motifs under accessible HMGB1 peaks; up- ( $>0.6 \log 2$ - fold change; orange) or downregulated TFs (<-0.6 $\log 2$-fold change; green $)$ are indicated.

(E) Box plots show the fold-change in mRNA levels $(\log 2)$ of genes differentially-expressed upon senescence and directly bound by HMGB1 in HUVECs (top) and IMR90 (bottom). The most enriched GO terms associated with each subgroup and their enrichment $P$-values are shown. The number of peaks $(N)$ analyzed is indicated below each bar. 
Results

A

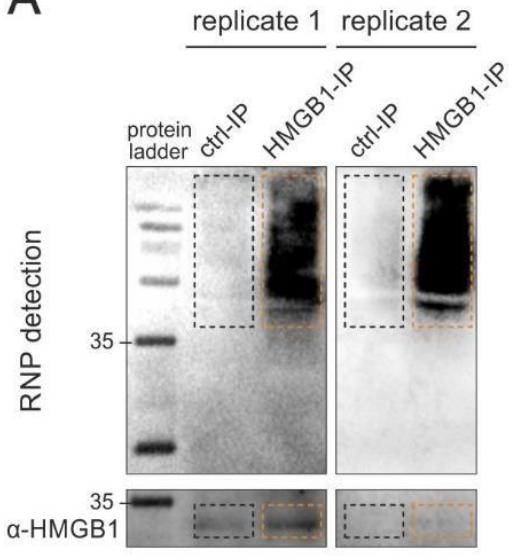

C

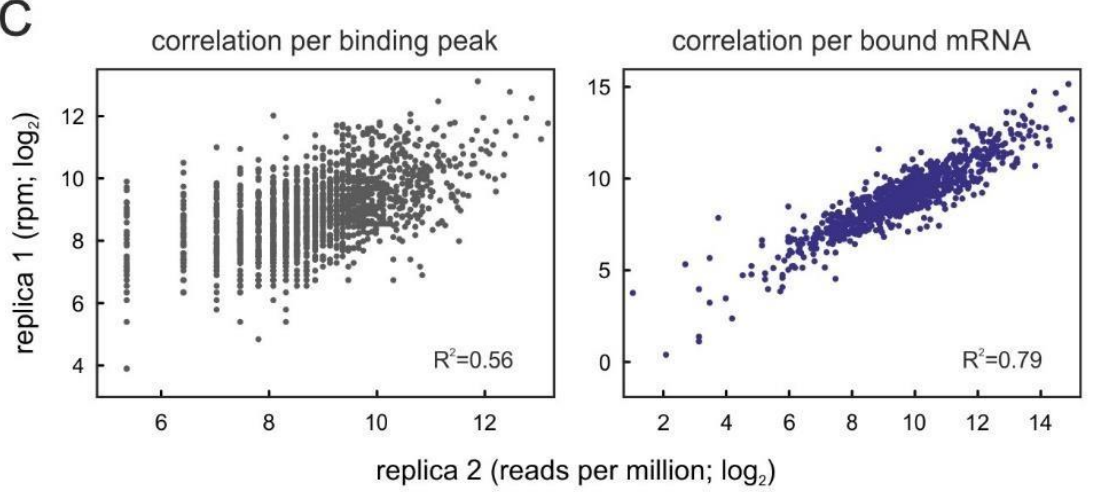

D

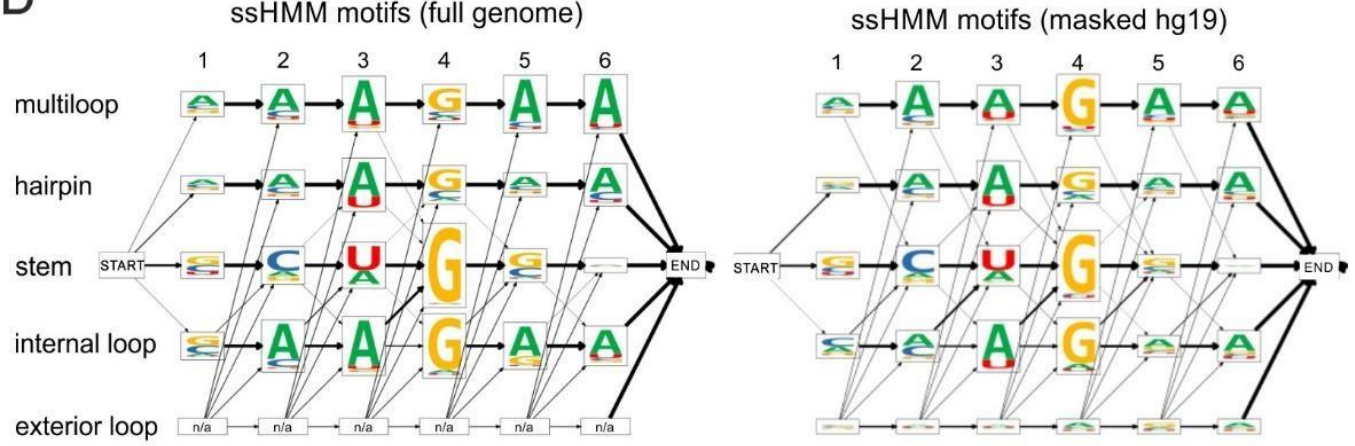

E

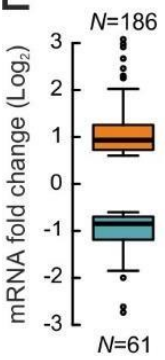

B

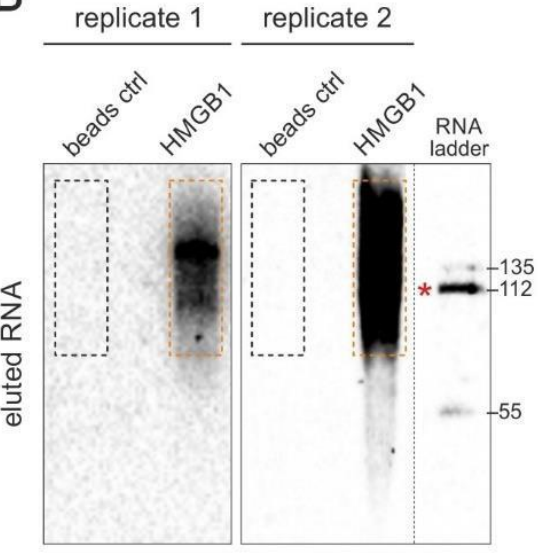


Figure 3.2.S4. HMGB1 sCLIP controls and analysis.

(A) Electrophoretic profiles of control (beads only; black dotted square) and HMGB1 IP (orange dotted rectangle) probed for RNA (top) or the HMGB1 protein (bottom) in both sCLIP replicates. The 35-kDa band of the molecular mass ladder is indicated.

(B) Electrophoretic profiles of RNA eluted from control (beads only; black dotted square) or HMGB1 IP (orange dotted rectangle) in both sCLIP replicates. The 112-nt band of the molecular mass ladder is indicated (red star) and corresponds to $\sim 144$ pg of RNA.

(C) Scatter plots show the correlation of sCLIP data from two independent biological replicates compared per binding peak (left) or per bound mRNA normalized read count (right). Spearman correlation values $\left(\mathrm{R}^{2}\right)$ are indicated.

(D) Output of ssHMM motif analyses of sCLIP data showing sequence probabilities for HMGB1-bound hexameric motifs predicted to form different structures using either the complete (top) or masked reference human genome (bottom; hg19).

(E) Box plots show fold-change in expression $(\log 2)$ of mRNAs differentially-regulated upon senescence and directly bound by HMGB1 in IMR90 sCLIP. The most enriched GO terms associated with each subgroup and their enrichment $P$-values are shown. The number of peaks $(N)$ analyzed is indicated below each bar. 

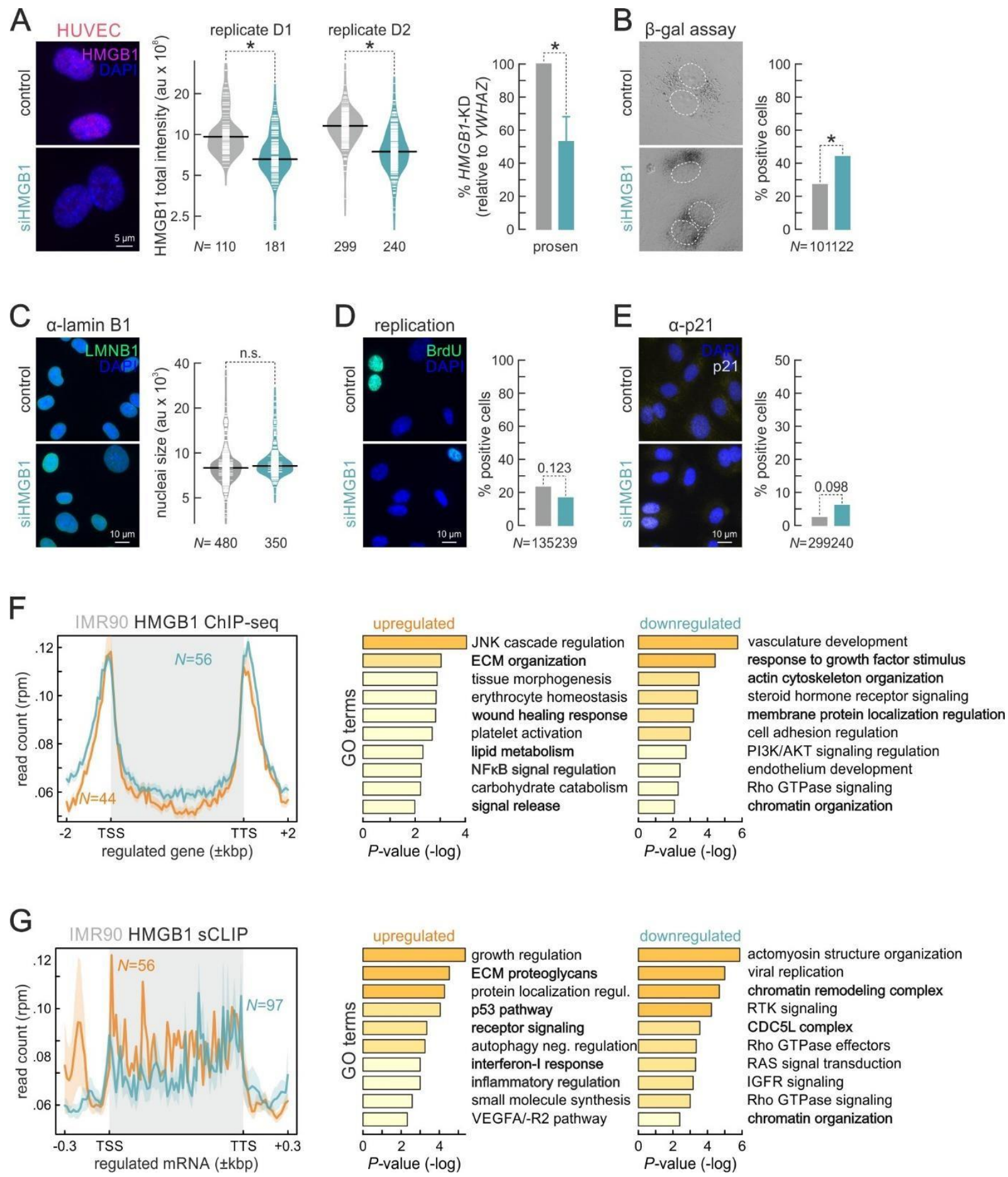

Figure 3.2.S5. Effects of HMGB1 knockdown in proliferating HUVECs and IMR90.

(A) Representative immunofluorescence images of siHMGB1-treated HUVECs show reduced HMGB1 levels compared to control cells (left), and bean plots quantify knockdown efficiency (middle; $N$ indicates the number of cells analyzed). Bar plots (right) show normalized $H M G B 1$ mRNA levels ( \pm S.D.; $N=2)$ in knockdown compared to control cells. Bar: $5 \mu \mathrm{m}$. *: $P<0.01$; Wilcoxon-Mann- Whitney and unpaired two-tailed Student's t-test for bean and bar plots, respectively.

(B) Representative brightfield images of siHMGB1-treated HUVECs show elevated $\beta$-galactosidase activity levels compared to control cells (left), and bar plots quantify this increase (right; $N$ indicates the number of cells analyzed). *: $P<0.01$; Fisher's exact test. 


\section{Results}

(C) As in panel A, but for LMNB1 levels (left), and bean plots quantify this (right; $N$ indicates the number of cells analyzed). Bar: $10 \mu \mathrm{m}$. No statistically significant difference (n.s.); Wilcoxon- Mann-Whitney test.

(D) As in panel A, but for BrdU incorporation (left), and bar plots quantify BrdU-positive cells (right; $N$ indicates the number of cells analyzed). Bar: $10 \mu \mathrm{m} . P=0.123$; Fisher's exact test.

(E) As in panel A, but for p21 levels (left), and bar plots quantify p21-positive cells (right; $N$ indicates the number of cells analyzed). Bar: $10 \mu \mathrm{m} . P=0.098$; Fisher's exact test.

(F) Line plots (left) show the mean distribution of IMR90 HMGB1 ChIP-seq signal along gene bodies of genes up- (orange) or downregulated upon HMGB1 knockdown (green; $N$ indicates the number of directly HMGB1bound genes). Bar plots show the most enriched GO terms associated with either gene set and their enrichment $P$-values (middle/right), and those relevant to senescence are highlighted (bold).

(G) As in panel C, but using IMR90 HMGB1 sCLIP data 
Results

3.2 Chapter III - Repurposing a small-molecule HMGB inhibitor to induce rapid and uniform SASPless senescence

This project was conceived and coordinated by Dr. A. Papantonis. The manuscript was written by Konstantinos Sofiadis and Dr. A. Papantonis. I was involved in the majority of the experimental procedures. Natasa Josipovic performed all the bioinformatic analyses. Nadine Übelmesser and Spyridon Palikyras performed proliferation assays and part of the experimental procedures. 


\section{Repurposing a small-molecule HMGB inhibitor to induce rapid and uniform SASPless senescence}

Konstantinos Sofiadis ${ }^{1,2,}$, Natasa Josipovic ${ }^{1,2,}$, Nadine Übelmesser ${ }^{1}$, Spyridon Palikyras ${ }^{1}$, Janine Altmüller ${ }^{2}$, Peter Nürnberg ${ }^{2} \&$ Argyris Papantonis ${ }^{1}$

${ }^{1}$ Institute of Pathology, University Medical Center Göttingen, 37075 Göttingen, Germany

${ }^{2}$ Cologne Center for Genomics, University of Cologne, 50931 Cologne, Germany

§.\#These authors contributed equally to this work

\subsubsection{Abstract}

Cellular senescence is a state of permanent cell cycle arrest that acts as a potent tumour-suppressive mechanism. However, recent evidence links senescence to age-associated diseases, including to more permissive tumour development. A major role in this homeostatic imbalance is attributed to the SASP, the dynamic proinflammatory secretome of senescent cells. Fine tuning of the balance between senescence induction and SASP magnitude is important to counteract the development of senescenceassociated pathophysiological phenotypes. Here, we are repurposing a small-molecule antiinflammatory agent, inflachromene (ICM), previously described to block HMGB eviction from the nucleus and show that it irreversibly induces SASPless senescence within few days. This coincides with a blockage of autophagic flux mediated by the ICM-driven alteration of HMGB1 kinetics. Critically, ICM also has an antiproliferative effect on cancer cells that are addicted to high HMGB titers. 


\subsubsection{Introduction}

Cellular Senescence is a natural barrier against the aberrant proliferation of malignant cells (Campisi, 2013). Although its initial definition describes a potentially beneficial process for cellular homeostasis, accumulating evidence render senescence a hallmark of organismal aging (López-Otín et al., 2013). One of the major features of cellular senescence, and a key contributor to organismal aging, is the senescence-associated secreted phenotype (SASP), as a result of which numerous pro-inflammatory cytokines, chemokines, and growth factors are secreted from senescent cells (Coppé et al., 2010). The beneficial role attributed to the SASP involves wound healing and immuno-clearance of senescence cells (Watanabe et al., 2017), whereas extensive and chronic inflammation due to senescent cell accumulation in tissue contributes to the progression of age-related diseases and tumour development (Coppé et al., 2010). In fact, it was shown that targeted elimination of senescent cells either by genetic manipulations (Baker et al., 2011; Jeon et al., 2017) or via administration of senolytic drugs (Chang et al., 2016; Xu et al., 2018; Yosef et al., 2016) increases health- and lifespan of mice. Thus, controlling the secretion of SASP factors and specifically target the senescent cells represents an era of senolytic drugs development (van Deursen, 2019).

Various factors have been described as contributors of senescence induction, including the high mobility group proteins (HMGBs) (Davalos et al., 2013; Sofiadis et al., 2019; Zirkel et al., 2018). HMGBs are highly abundant in proliferating cells, but also actively evicted from the nuclei of cells entering senescence (Davalos et al., 2013; Sofiadis et al., 2019; Zirkel et al., 2018). In fact, the sub-cellular localization of HMGBs and their relative levels determine the function of these versatile proteins. Notably, in the nucleus of proliferative cells, HMGBs possess a dual function by acting as both architectural proteins capable of increasing the accessibility of transcription factors to enhance the transcriptional output (Štros, 2010), and as regulators of nascent and mature RNAs (Sofiadis et al., 2019). Finally, for cellular senescence HMGB1 is actively secreted into extracellular space, where it can diffuse and bind to TLR and RAGE receptors acting as a key SASP factor (Davalos et al., 2013). Thus, manipulation of the sub-cellular localization of HMGBs could affect multiple pathways with senescence and SASP being among them.

Recently, a small benzopyranyl tetracycle drug called inflachromene (ICM) was introduced as an anti-inflammatory agent blocking the translocation of HMGBs from cell nuclei by altering their posttranslational modification state (Lee et al., 2014). Increased nuclear levels of HMGBs suppress spreading of microglia-mediated inflammation and ameliorate the effects of experimental autoimmune encephalomyelitis (EAE) and sepsis (Cho et al., 2017; Lee et al., 2014). However, the exact mechanism that determines the fate of HMGB1 in the absence of translocation, the subsequent diminished inflammation and the long-term impact of ICM on primary human cells remains elusive. 
Contrary to previous data, we report here that just a few days of ICM treatment irreversibly block proliferation of human primary cells and induce a homogeneous senescence-like program. We can show that the induction of this type of senescence is due to the global HMGB1/2 loss rather than due to a block of its translocation to the cytoplasm. This loss also leads to diminished autophagic flux. Furthermore, we show that the ICM-induced senescence (IIS) lacks SASP, due to poor HMGB1 secretion and loss of HMGB1-mediated paracrine signaling. We also suggest that these changes attributed to ICM are mediated by alterations in the chromatin architecture due to the ICM-modulation of HMGB kinetics. Taken together, ICM represents a useful tool for manipulating HMGB1/2 to such an extent that cancer cells addicted to high HMGB titers can be selectively killed by this small-molecule agent.

\subsubsection{Results}

\section{ICM triggers a replicative senescence-like transcriptional program}

Inflachromene was previously reported as a selective inhibitor of HMGB nuclear eviction (Lee et al., 2014). We hypothesized that an ICM-mediated block of HMGBs export might prove sufficient to block or stall senescence induction. To test this, we obtained two distinct primary human cell types previously used in our senescence studies from individual donors/isolations: human umbilical vein endothelial cells (HUVECs; mesodermal) from two single donors and two isolates of fetal lung fibroblasts (IMR90s; endodermal). Surprisingly, treatment with previously reported concentrations of ICM (5 and $10 \mu \mathrm{M})$ rapidly arrested population growth of both cell types even after treatment for as few as three days. Since we did not observe cell death, we sought to decipher whether lower cell numbers were due to cell cycle arrest. We cultured equal number of DMSO- and ICM-treated cells and confirmed the gradual drop of proliferation rates (Figure 3.3.1A), which persisted even after ICM removal (data not shown). Beta-galactosidase staining showed positive staining in the ICM- treated cells (Figure 3.3.1B), indicating that the cell cycle arrest is possibly the result of ICM-induced senescence (ISS). Fluorescence-activated cell sorting (FACS) of cells stained with PI to differentiate for the various stages of the cell cycle showed that ICM induces an S-phase arrest (Figure 3.3.1C).

Although $\beta$-galactosidase staining is extensively used as a senescence marker, it does not allow for a confident characterization of senescence. Therefore, we decided to interrogate the transcriptional profiles of IMR90s at different lengths of ICM treatment looking for a senescence signature. To this end, we used a previously described method that isolates RNA from the sites of active transcription and we performed “factory" RNA-seq (Melnik et al., 2016). We next compared this factory RNA-seq data with genes previously identified as differentially-expressed upon entry into replicative senescence (Zirkel et al., 2018). Direct comparison of the two datasets resulted a high correlation of a large number of genes between ICMtreated and replicative senescence conditions (Figure 3.3.1D). Finally, we intersected differentially expressed genes from both the 3- and 6-day time points to check for potential differences and similarities. 
We found a large number (>1100) of shared down-regulated genes (Figure 3.3.1E). Gene-ontology (GO) analysis of this shared gene set also generated a pronounced senescence signature (cell cycle, cell division, DNA replication) confirming cell cycle arrest due to IIS.

A
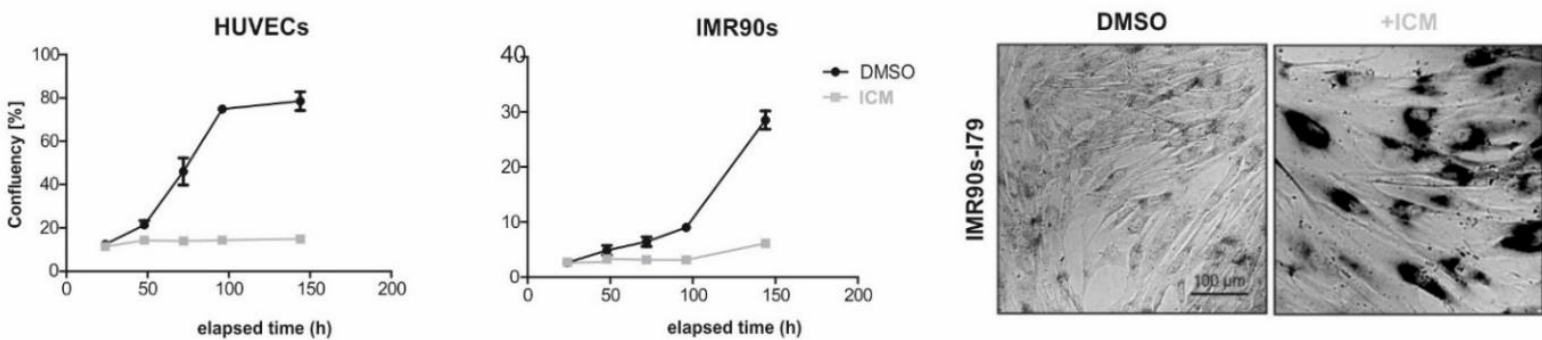

B
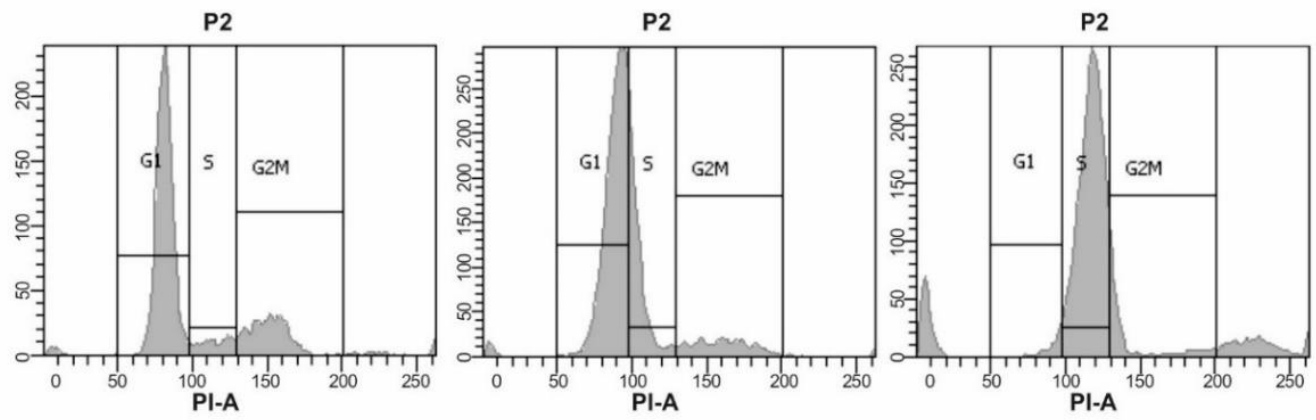

C
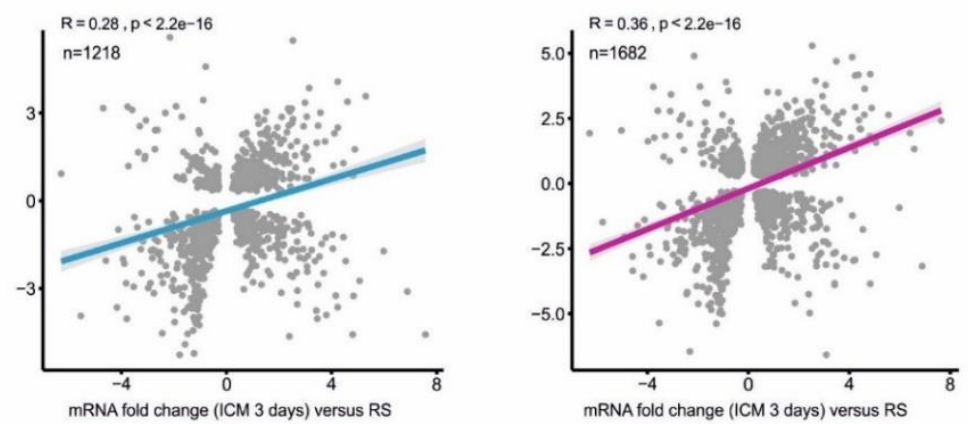

D

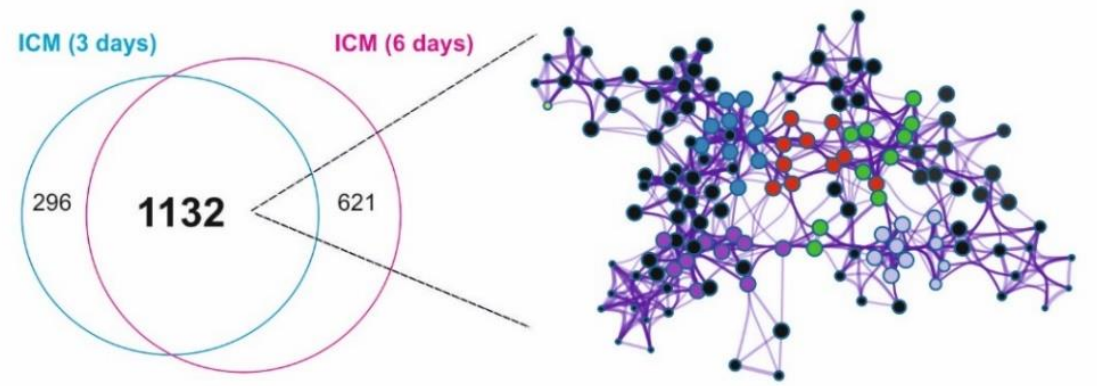

Cell cycle, mitotic

DNA conformation change

DNA replication

Cell division

Other

Page | 120 
Figure 3.3.1. ICM promotes cell-cycle in human cells

(A) Control and ICM-treated cells were tested for proliferation rates through Celigo Image Cytometer (left) and $\beta$ galactosidase activity (right)

(B) Cell cycle progression was measured by FACS analysis

(C) Plots showing differentially expressed mRNAs $\left( \pm 0.6-\log 2\right.$ fold change or more; $\left.\mathrm{P}_{\mathrm{adj}}<0.05\right)$ after RNA-seq data normalization for IMR90 factory RNA three and six days and IMR90 mRNA from replicative senescence (RS).

(D) Overlap of down-regulated genes across IMR90s treated with ICM for three and six days (left). GO analysis shows pathways associated with downregulated genes. Nodes containing genes involved in cell cycle, DNA conformation change, DNA replication, cell division are highlighted 


\section{ICM-induced HMGB loss triggers chromatin changes}

Nascent RNA-seq from ICM-treated cells presented profiles similar to replicative senescence with both HMGB1 and -2 strongly downregulated. Since ICM was claimed to be a potent inhibitor of their nuclear export (Lee et al., 2014), we asked whether ISS induction is dependent on HMGB nuclear levels. To test this, we performed immunofluorescence stainings and Western blots to assess HMGB levels and subcellular localization at different time-points. Interestingly, both approaches showed progressive loss of HMGB1 and -2 from the nucleus of ICM-treated cells in sharp contrast to the initial report regarding ICM (Kim et al., 2018; Lee et al., 2014) (Figure 3.3.2A-B). This nuclear loss of HMGBs was accompanied by the emergence of key senescent markers (Figure 3.3.2B).

We previously documented the functional versatility of HMGBs in the nucleus of proliferating cells and their impact on senescence entry (Davalos et al., 2013; Narita et al., 2006; Sofiadis et al., 2019; Zirkel et al., 2018) RNA-seq comparisons highlight a clear subset of architectural chromatin proteins (Figure 3.3.1E) rapidly decreasing transcriptional even upon only three days of ICM treatment. Similarly, western blotting confirmed that changes in the levels of different chromatin-associated factors coincides with the emergence of senescence (Figure 3.3.2B). We sought to find IIS is also marked by the previously reported hallmark of replicative senescence, the formation of senescence-induced CTCF clusters (SICCs) or the hallmark of oncogene-induced senescence, the formation of senescence associated-heterochromatin foci (SAHF) (Narita et al., 2003). Indeed, 3-day ICM treatment was sufficient to induce SICCs in almost half of the cell population, a phenotype that becomes almost uniform across cells after longer exposure (Figure 3.3.2C). Similarly, albeit to less extent, closer inspection of the DAPI distribution in the nucleus shows a gradual increase of a puncta formation in the nuclei of ICM-treated cells. Interestingly this punctuated DAPI-staining overlaps dense H3K9me3 clusters, suggesting a clear redistribution of the heterochromatin following ICM treatment that is reminiscent of, but not identical to, SAHF formation (Figure 3.3.2E). 
A
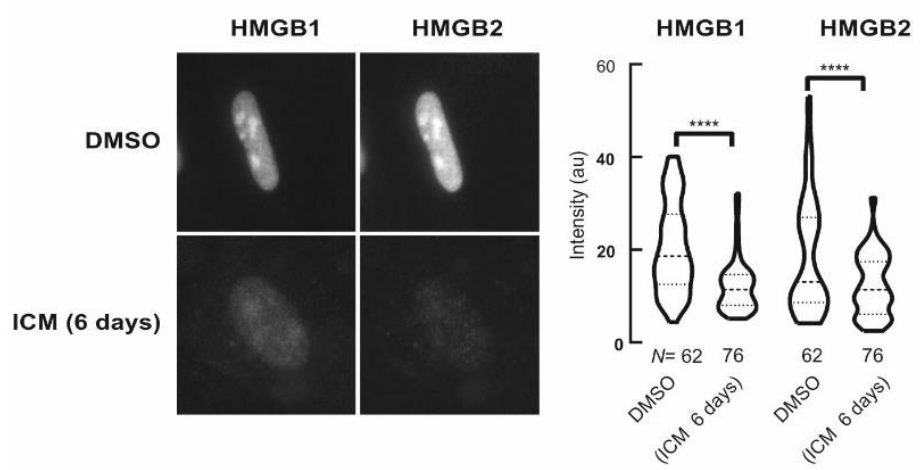

C

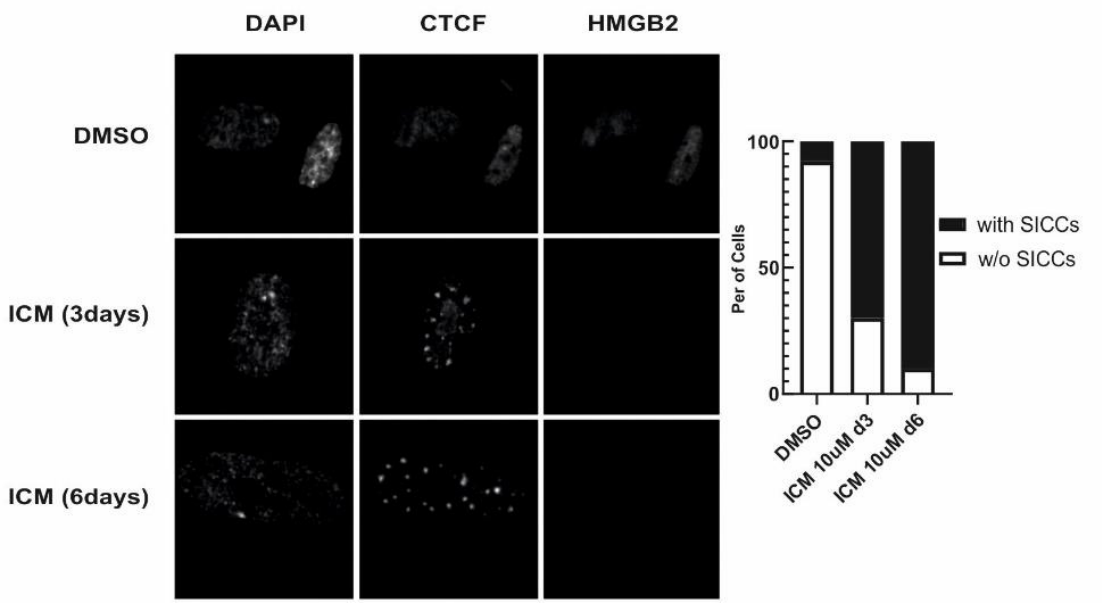

B

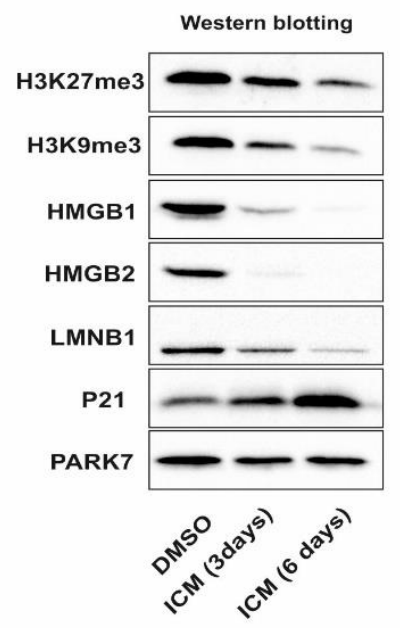

D

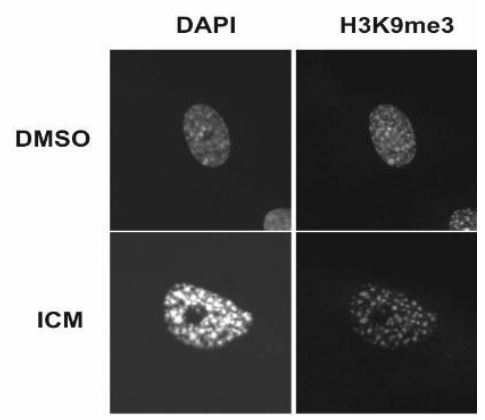

Figure 3.3.2. ICM induces chromatin changes

(A) Representative immunofluorescence images of IMR90 show reduced HMGB1 and HMGB2 levels in ICM treated cells (left) and bean plots quantify this reduction (right; $N$ indicates the number of cells analyzed per condition)

(B) Western blots show changing protein levels in IMR90 upon ICM treatment

(C) Representative immunofluorescence images of IMR90 show accumulation of SICC and decrease HMG2 levels in ICM treated cells (left) and bar plots quantify the number of cells with and w/o SICC

(D) Representative immunofluorescence images of IMR90 show punctuated formation of DAPI and H3K9me3 upon ICM treatment 


\section{IIS induction coincides with diminished autophagic flux}

Since ICM was recently documented to block autophagic flux (Kim et al., 2018) and our factory RNAseq data contain a subset of differentially-expressed genes associated to macroautophagy genes, we asked whether IIS induction is linked to suppressed autophagy. Whole-cell label-free proteomics revealed strong upregulation of autophagy-related proteins (Figure 3.3.3A). Generally, autophagy progression is mediated by the fusion of autophagosomes with autolysosomes to form lysosomes and load their cargo for degradation (Nakamura and Yoshimori, 2017). To monitor the autophagic flux we an LC3 dual fluorescent rerporter that senses $\mathrm{pH}$ changes during the transition of neutral autophagosomes into acidic autophagolysomes. We observed robust accumulation of LC3-positive signal in ICM-treated cells compared to the control population, and this signal completely disappeared upon longer treatment with the drug (Figure 3.3.3B). Accumulating LC3 punctae were makred by extensive overlap between the GFP and RFP in the LC3-positive cells indicating block of autophagic flux. Similarly, while total LC3 levels remained constant upon ICM treatment, the absence of oxidized LC3B in the western blots confirmed the absence of even bassaly-activated autophagy. To further confirm this ICM-mediated block of autophagy, we used a panel of different antibodies related to autophagy in western blots. Interestingly, the highest expression for the majority of these factors was at day 3, while longer exposure to ICM restored LC3 expression levels (Figure 3.3.3C). Furthermore, we observed strong accumulation of p62 in both western blots and immunofluorescence images, whereas ATG7 levels decreased upon ICM treatment and ATG5 ones remained unaffected. Finally, the characterized target of HMGB1 (Kang et al., 2010), Beclin 1 remained constant as also previously reported (Figure 3.3.3C).

Since our data suggested ICM-mediated accumulation of LC3 signal, but no further progression of autophagy, we focused on p62, the major regulator of autophagosomes. Our recent work where we performed Clip against HGMBs revealed p62 was a direct target of HMGB1 but not HMGB2 (Sofiadis et al., 2019). This is in line with the characterized role of HMGB1, but not of HMGB2, in the regulation of autophagy. We stained ICM-treated cells with HMGB1 and p62 and observed strong emergence of p62 punctae specifically in HMGB1-depleted cells. In fact, the accumulation of p62 signal was proportional to the concentration of ICM used, but inversely correlated to HMGB1 nuclear levels. To gain further insight in the HMGB1-p62 interplay, we knocked-down HMGB1 and assessed the expression levels of several autophagy factors. Interestingly, we observed upregulation of p62 and mTOR, which represent upstream mediators of autophagy. Taken together, ICM induces an autophagic block in primary human cells by altering the levels and kinetics of HMGB1.

Autophagy and translation share upstream effectors, and the increase of mTOR led us to hypothesize that blocking autophagy might lead to a subsequent lack of nutrients affecting the translational rates. Thus, we generated the first genome-wide ribosome profiling for replicative senescence, in parallel to that of IIS. However, despite the pronounced autophagy block, we did not 
observe changes that specifically concerned translation, as any changes seen in polysome-associated footprints was essentially always accompanied by a respective change in mRNA abundance (Figure 3E). Thus, with very few exceptions, any observed changes in the protein abundance in either replicative or ICM-mediated senescence are not controlled at the translational level. Thus, IIS can be primarily attributed to autophagy blockage and transcriptional reorganization of cells in the absence of HMGBs.

A

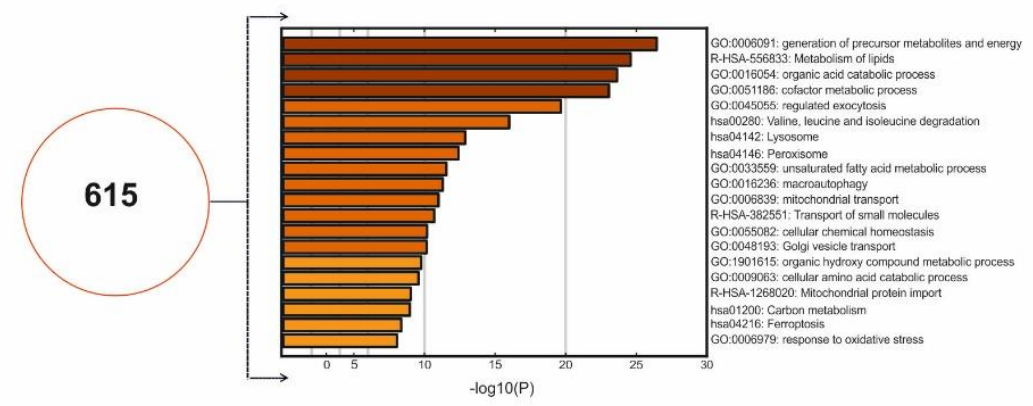

B

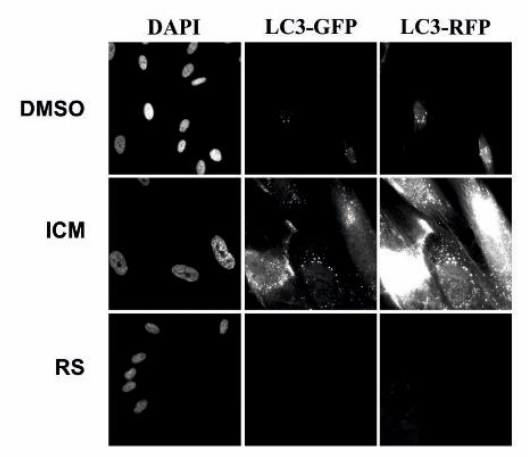

D

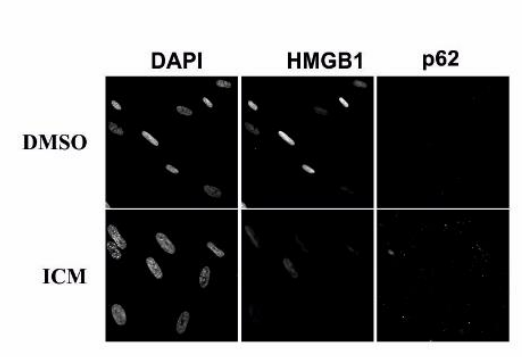

C

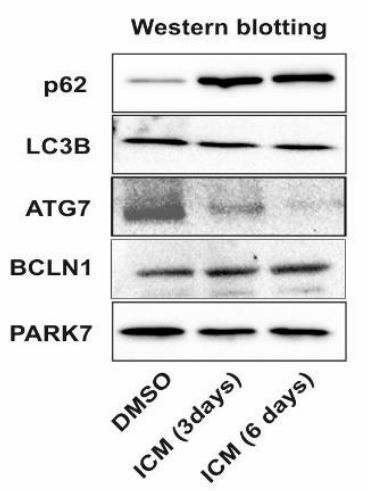

$E$

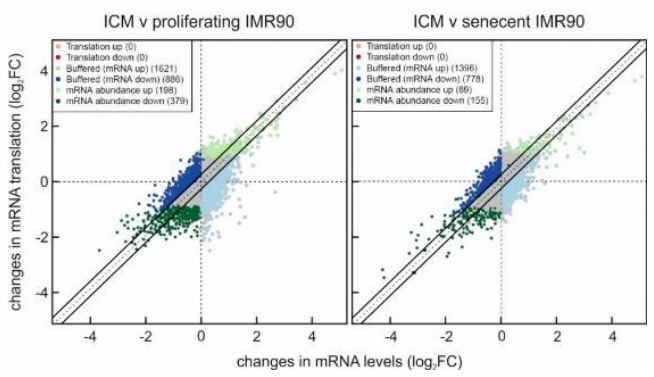

Figure 3.3.3. ICM autophagic flux

(A) Mass-spectrometry show of whole lysates shows 615 proteins down-regulated upon ICM treatment compare to DMSO (left). GO analysis shows pathways related to these proteins. The most enriched GO terms associated with each subgroup and their enrichment P-values are shown.

(B) Representative immunofluorescence images of IMR90 show accumulation of LC3b in the ICM-treated cells compare to DMSO and replicative senescent IM90s.

(C) Western blots show changes in the protein levels of autophagic protein upon ICM treatment 
(D) Representative immunofluorescence images of IMR90 show accumulation of p62 in the ICM-treated cells compare to DMSO-treated cells

(E) Plots showing a comparison differentially expressed mRNAs $\left( \pm 0.6-\log 2\right.$ fold change or more; $\left.\mathrm{P}_{\text {adj }}<0.05\right)$ and ribosome bound mRNAs after RNA-seq and Ribo-seq data normalization for ICM treated cells, proliferating and senescent IMR90).

\section{IIS is strongly depleted of proinflammatory SASP}

SASP represents one of the most striking features of senescence linked to both its beneficial and detrimental consequences(Coppé et al., 2010). ICM was initially described as an anti-inflammatory agent. Since SASP represents a bona fide response of senescence, we revisited transcriptomic profiles of IIS and RS cells and upon closer inspection saw that despite the significant overlap of genes associated with senescence, there exists a large subset of genes that are upregulated in the RS-but downregulated in ISS-cells. These genes gave GO terms directly linked to the SASP (e.g., MAPK signaling pathway, TNF/NF- $\kappa B$ signaling, response to LPS; Figure 3.3.4A). We validated the changed expression levels of marker SASP genes in IIS using RT-qPCR and observed that many interleukins and other SASP genes were downregulated upon 6-day ICM treatment, despite cells being fully senescent (Figure 3.3.4B). Apart from transcriptional regulation of the SASP pathway, we assessed secretion of proinflammatory factors. To this end, we collected the supernatant from TNF-treated cells co-treated with DMSO or ICM, while RS cells were used as controls for probing cytokine arrays. Despite limited sensitivity ranges, we could observe changes in some factors (Figure 3.3.1C; red boxes) confirming how IIS leads to cell cycle arrest devoid of major proinflammatory cues that characterize the SASP. 
A

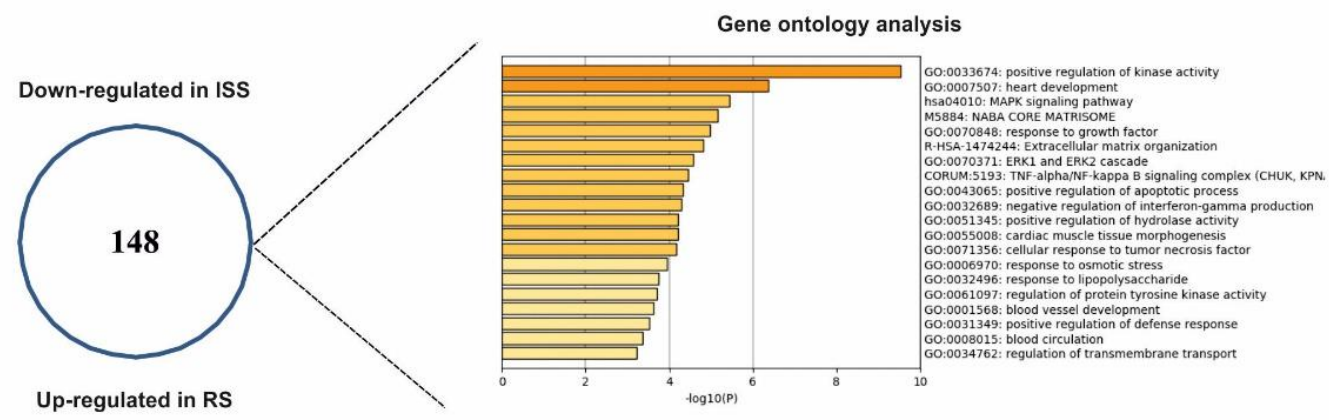

B

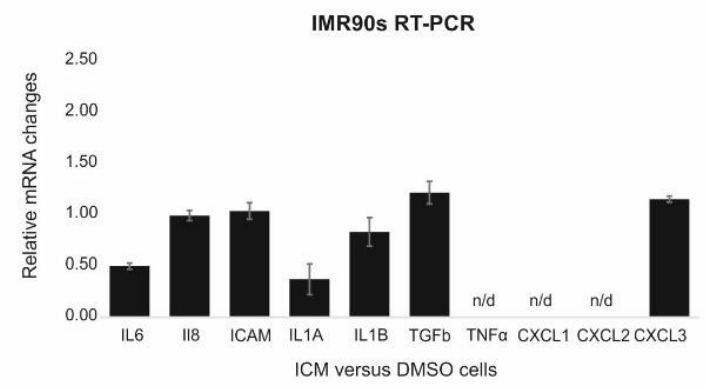

C

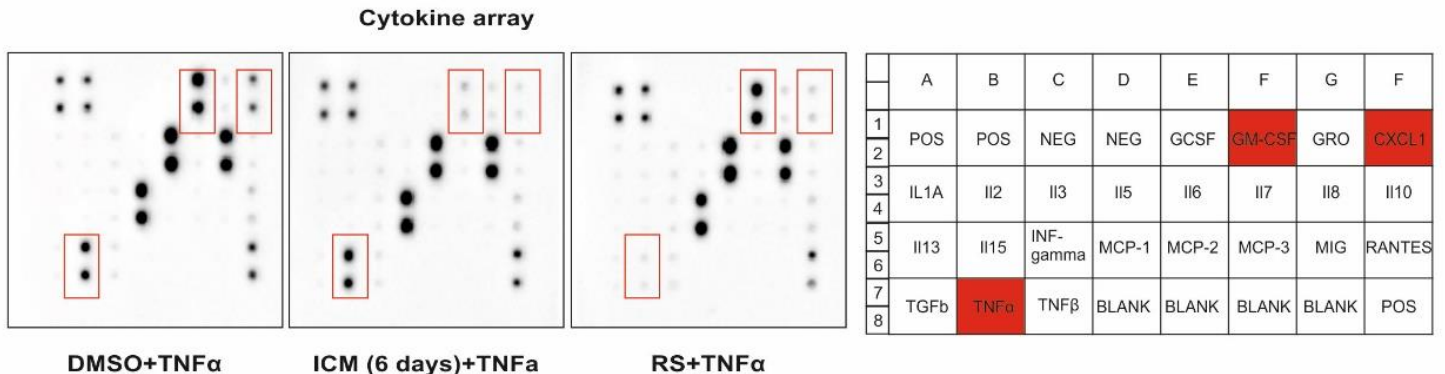

Figure 3.3.4. ICM induces a SASPless senescence

(A) Overlap of down-regulated genes and up-regulated across IMR90s treated with ICM and replicative senescent IMR90 respectively (left). GO analysis shows pathways associated with shared in both datasets. he most enriched GO terms associated with each subgroup and their enrichment P-values are shown.

(B) qPCR analysis (mean fold-change \pm S.D., $\mathrm{N}=3$ ) confirm the absence of SASP emergence upon ICM treatment

(C) Cytokine array showed the levels of multiple SASP factors in TNF $\alpha+$ DMSO and TNF $\alpha+$ ICM-treated IMR90 versus replicative senescence IMR90 


\section{ICM is potent inhibitor of cancer cell proliferation}

Nuclear loss of HMGB1 and its ensuing transcriptional changes are senescence hallmarks and represent an inherent antitumourigenic mechanism (Rai and Adams, 2013). However, HMGB1 is very often overexpressed in patient tumour specimens, and it has been suggested that its increased titers might result in either the enhancement of cell survival or the regulation of cell death via diverse pathways that include inflammation, cell proliferation, and autophagy (Nagatani et al, 2001; Kang et al, 2013). We focused on non-small cell lung adenocarcinoma patient-derived lines, because high HMGB1 expression therein is a predictor of poor patient survival. We used different lines, where cell proliferation and survival was significantly impaired following $H M G B 1$-knockdown for $72 \mathrm{~h}$. Interestingly, the extent of proliferative impediment in each line was almost proportional to its proliferation rate, with the faster H1975 cells arresting completely and the slower H1650 still exhibiting some population increase upon knockdown. Nonetheless, $H M G B 1$-knockdown in these lines showed convergent changes in the expression of senescence markers like HMGB2, LMNB1, EZH2 or SMC2, which are actually overexpressed in correlation to $H M G B 1$ levels in lung adenocarcinoma tumours (data from the TCGA cohort). Since knocking down HMGB1 in this context also decreased $H M G B 2$ levels, and we recently showed that reduced HMGB2 levels result in the formation of SICCs, we examined whether such CTCF clusters formed differentially in these lines. Indeed, we saw that the most affected H1975 cells exhibited larger and more prominent SICCs upon HMGB1-knockdown, compared to the least affected H1650 cells that essentially displayed no SICCs; the intermediately-affected A549 presented SICCs in both control and knockdown cells. Thus, differences in proliferative capacity and SICC emergence correlate with the anti-proliferative extent of $H M G B 1$ depletion in these cancer lines, highlighting how targeting HMGB1 might need to be considered in therapeutic interventions for cancer treatment. 
A

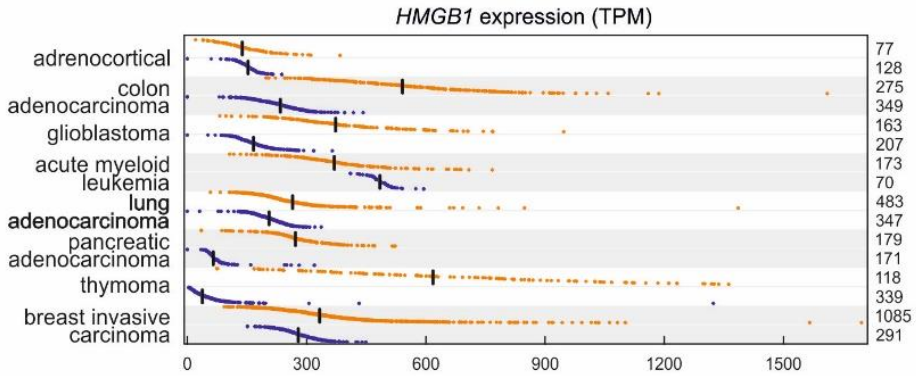

B
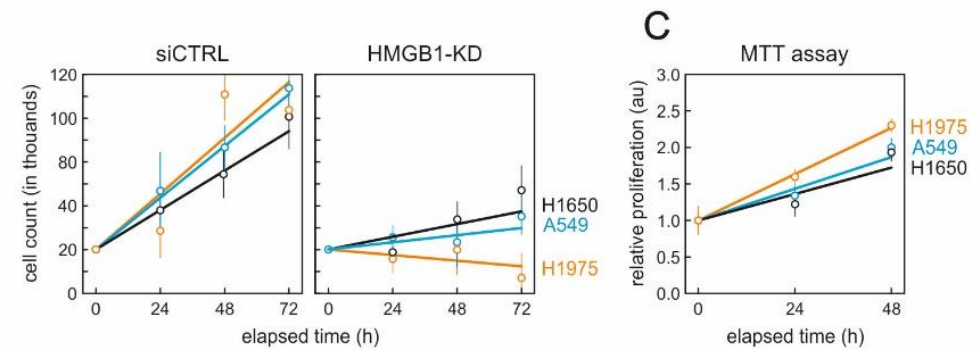

D
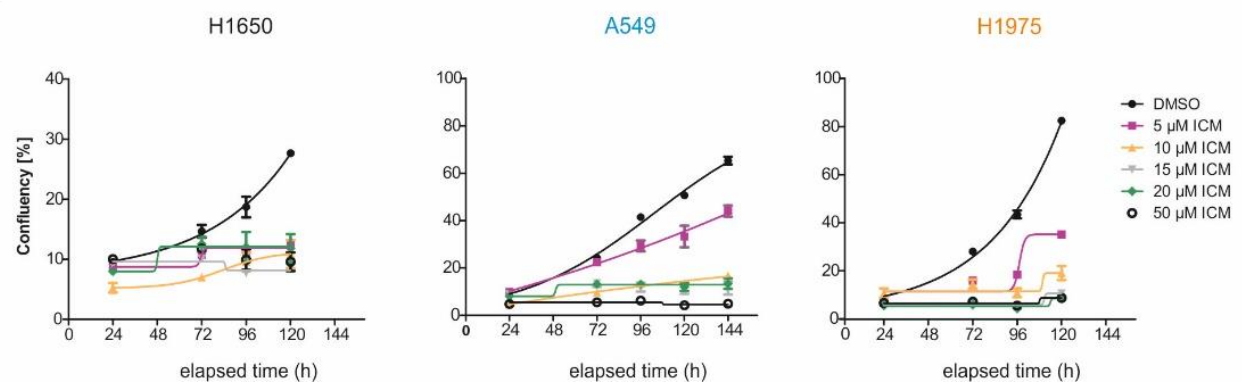

Figure 3.3.4. ICM induces a SASPless senescence

(A) Dot plots show HMGB1 expression levels (in transcripts per million) across individual patient samples from 8 cancer types (orange) matched to levels in normal tissue (blue; all data from the TCGA). Mean expression (black line) and number of samples per each type (right) are shown.

(B) Data points show mean changes ( \pm S.E.M.; N=3) in cell counts of control or HMGB1-knockdown H1975 (orange), A549 (blue), and H1650 lung adenocarcinoma cells (black) over $72 \mathrm{~h}$; trend lines are fitted using least mean square regression.

(C) As in panel B, but for relative proliferation rates of wild-type cells in MTT assays over $48 \mathrm{~h}$.

(D) Lung cancer cell types were tested for proliferation rates under different ICM concentration through Celigo Image Cytometer 


\subsubsection{Discussion}

The fragile balance between the beneficial effects of senescence as a tumour suppressive mechanism and its detrimental ones as a source of chronic inflammation (Coppé et al., 2010) highlights the need to develop strategies either to directly target senescent cells or to target the magnitude of their inflammatory secretion. An increasing number of studies focus on the development of senolytic drugs in order to address this need(Childs et al., 2015; Xu et al., 2018). On the other hand, we took advantage of a previously developed drug that is thought to target HMGBs and, thus, their important role in senescence, inflammation and tumour progression. Previous studies, including ours, highlighted the various roles of these proteins affecting multiple pathways in the different cellular sub-compartments and their paracrine role in the extracellular milieu upon the entry of cellular senescence (Davalos et al., 2013; Sofiadis et al., 2019; Zirkel et al., 2018).

The ability of ICM to target both HMGBs offers the possibility to interfere with various cellular processes and assess potential outcomes. We chose to repurpose ICM for our studies in order to alter the kinetics of these factors and potentially the emergence of the senescent phenotype. Intriguingly, 10 $\mu \mathrm{M}$ of ICM lead to very similar profiles to that of RS, thereby arresting cell proliferation within as few as 3 days. Transcriptomic analyses revealed extensive overlap between IIS and RS as regards pathways strongly linked to cell cycle and genome architecture regulation. Prominent factors of this latter group are the HMGBs, and we showed that this transcriptional shutdown is actually coupled to their decreased nuclear levels (in contrast to initial ICM reports). The absence of HMGBs from the IIS nuclei results in altered heterochromatin and lamin levels, much reminiscent of RS (Sofiadis et al., 2019; Zirkel et al., 2018).

However, despite general convergence, our transcriptomic comparison also showed that in IIS macroautophagy and SASP gene expression are up- and down-regulated compared to levels in RS, respectively. As previous reports showed that ICM inhibits the interaction of HMGB1 with Beclin (Lee et al., 2014) that would induce autophagy (Kang et al., 2010), we characterized this more to show that the initial cascade favouring autophagy is highly activated with the production of high levels of autophagosomes, perhaps precisely to compensate for the autophagy block imposed. This autophagic blockage coincides with the gradual loss of the HMGBs from cell nuclei and the emergence of the key senescent markers so we believe that the depleted autophagy is a key stimulus for IIS. Still, the main effect here is that this loss of HMGB1 (and HMGB2) sequesters the initiating paracrine effects that induce SASP in the first place. This is important, as it renders ICM a potent inhibitor capable of inducing cell-autonomous senescence independent of any of the side effects of inflammation. Moreover, it generates essentially uniformly senescent cell populations within 3-6 days that can now allow for the study of the core senescent program and its implementation (compared to the highly heterogeneous and SASP-rich RS populations). 
Finally, we took advantage of three lung carcinoma cell lines differentially influenced by HMGB1 levels to show that ICM suffices to decrease their proliferation rate an effect that is correlated to the expression levels of HMGB1. Thus, we further propose ICM as a selective inhibitor of aberrant cell proliferation through the modulation of HMGBs and without the caveats of overt inflammatory phenotypes. It is not impossible to then imagine ICM as a pro-senescence cancer treatment in combination with other chemotherapy agents, and this indeed merits further work.

\subsubsection{Materials and methods}

\section{Primary human cell lines and senescence markers}

HUVECs from single, apparently healthy, donors (passage 2; Lonza) were were grown in complete Endopan-2 supplemented with 2\% FBS under 5\% CO2. Cells were constantly seeded at _10,000 cells $/ \mathrm{cm}^{2}$, except late passages that were seeded at $\sim 20,000$ cells $/ \mathrm{cm} 2$. Single IMR90 isolates (I90-10 and -79, passage 5; Coriell Biorepository) were grown in MEM (M4655, Sigma-Aldrich) supplemented with non-essential amino acids and 10\% FBS under 5\% CO2. Lung cancer cells were grown to RPMI 1640 medium (Gibco) supplemented with 10\% FBS. Inflachromeme was added for 3 and 6 days in concentrations of 5 and $10 \mu \mathrm{M}$ for downstream applications. Cell proliferation was monitored by Celigo Image Cytometer after culturing 2000 cells for every sample in triplicates followed by $10 \mu \mathrm{M}$ ICM and DMSO treatment for 6 days. Measurements of the cell confluency were obtained every day. Bgalactosidase assay was used followed the manufacture's instructions (Cell signaling).

\section{$\underline{\text { Immunofluorescence and image analysis }}$}

Cells were grown on coverslips from the stage indicated; they were fixed in 4\% PFA/PBS for $15 \mathrm{~min}$ at room temperature. After washing once in PBS, cells were permeabilized with $0.5 \%$ Triton-X/PBS for $10 \mathrm{~min}$ at room temperature. Blocking with $1 \% \mathrm{BSA} / \mathrm{PBS}$ for $1 \mathrm{~h}$ was followed by incubation with the indicated primary antibodies for 1-2 h. Cells were washed twice with PBS for 5 min before incubating with secondary antibodies for $1 \mathrm{~h}$ at room temperature. Nuclei washed three times with PBS for $10 \mathrm{~min}$ and coverslips mounted onto slides in Prolong Gold Antifade with DAPI (Invitrogen). For image acquisition, a widefield Leica DMI 6000B with a HCX PL APO 63x/1.40 (Oil) objective was used.. LC3 stainings were performed with Premo ${ }^{\mathrm{TM}}$ Autophagy Tandem Sensor RFP-GFP-LC3B Kit according to the manufacturer's instructions.

\section{Protein extraction and western blotting}

For assessing protein abundance at the different cell states, approx. $4 \times 10^{6}$ cells were gently scraped off 15-cm dishes, and pelleted for $5 \mathrm{~min}$ at $600 \mathrm{x} \mathrm{g}$. The supernatant was discarded, and the pellet resuspended in $100 \mathrm{~mL}$ of ice-cold RIPA lysis buffer $(20 \mathrm{mM}$ Tris- $\mathrm{HCl} \mathrm{pH} 7.5,150 \mathrm{mM} \mathrm{NaCl}, 1 \mathrm{mM}$ EDTA pH 8.0, 1 mM EGTA pH 8.0, 1\% NP-40, 1\% sodium deoxycholate) containing 1x protease 
inhibitor cocktail (Roche), incubated for $20 \mathrm{~min}$ on ice, sonicated for 3 cycles (30 sec on, $30 \mathrm{sec}$ off, low input) and centrifuged for $15 \mathrm{~min}$ at $>20,000 \mathrm{x}$ g to pellet cell debris and collect the supernatant. The concentration of the nuclear extracts was determined using the Pierce BCA Protein Assay Kit (Thermo Fisher Scientific), before extracts were aliquoted and stored at $-80^{\circ} \mathrm{C}$ to be used for western blotting. Resolved proteins were detected using the antisera and dilutions listed in the Key Resources Table.

\section{$\underline{\text { RNA isolation, sequencing, and analysis }}$}

Cell of different types/conditions were harvested in Trizol LS (Life Technologies) and total RNA was isolated and DNase I-treated using the Direct-zol RNA miniprep kit (Zymo Research). Following, rRNA-depletion using the RiboZero Gold kit (Illumina), barcoded cDNA libraries were generated using the TruSeq RNA library kit (Illumina). The resulting libraries were paired-end sequenced to at least 50 million read pairs on a HiSeq4000 platform (Illumina), and raw reads were mapped to the human reference genome (hg19) and analyzed via the QuickNGS pipeline (Wagle et al., 2015) to obtain read counts. Then, read counts from different single-donors/conditions were normalized using the RUVSeq package (Risso et al., 2014). Please note that, in our hands, normalization using either synthetic spikein controls or the intrinsic " top quantile" function of the RUVseq package is essentially interchangeable. Finally, for analysis of nascent RNA in IMR90 the "factory RNA-seq" approach was applied on _5 million proliferating or ICM treated cells (Melnik et al., 2016), RNA was isolated and sequenced as above, and intronic read counts were obtained and differentially analyzed for the two conditions using the iRNAseq package (Madsen et al.,2015). All box/bean plots were plotted using the BoxPlotR tool online (http://shiny.chemgrid.org/boxplotr/), and GO term/pathway enrichment analyses using Metascape (http://metascape.org/gp/index.html; Tripathi et al., 2015). Differentially-regulated genes per each cell type are listed in Table S2. Ribo-seq was permormed in collaboration with RIBO-MAPS. We isolated the RNA from proliferating, ICM treated and replicative senescent with polysome buffer and sequenced.

\section{Statistical tests}

P-values associated with Student's t-tests and Fischer's exact tests were calculated using GraphPad (https://graphpad.com/), and those associated with the Wilcoxon-Mann-Whitney test using the U test interface (https://www.socscistatistics.com/tests/mannwhitney/Default2.aspx). Unless otherwise stated, P-values $<0.01$ were deemed as statistically significant. 


\section{Discussion}

The coordinated activation and execution of DNA replication and cell division in proliferating cells is governed through inherent mechanisms that reassure the expansion of the cell population in a controlled manner. These mechanisms are also responsible for the re-establishment of the cell signature and the progression of the respective functions of the newly synthesized cells to preserve the normal homeostasis. During mitosis the chromatin folding heavily dissolves, the architectural features disappear and the condensed chromosomes acquire a homogeneous folding state (Gibcus et al., 2018; Naumova et al., 2013). The three-dimensional genomic organization immediately emerge upon the re-entry of daughter cells in interphase to segregate the genome in the active euchromatin and the repressed heterochromatin, shaping the transcriptional landscape. On the other hand, senescent cells are devoid of cellular division and the mechanisms underlying their genomic organization in the arrested state are still elusive. Up to date, multiple changes related to chromatin have been described to accompany senescence and whether these changes comprise cause, consequence or a concurrent event in respect to the emergence of the classical senescence pathways and the establishment of the cell cycle arrest is yet to be described. Here, we attempt to understand some of the rules shaping the genomic organization during senescence and particularly the events promoting the transition from the proliferating state to the arrested one. We are focusing to the architectural proteins, HMGB1 and HMGB2, abundantly present in the nucleus of growing cells, but dynamically translocated in the extracellular space during senescence and we describe the impact of this translocation on the chromatin folding and the transcriptional programs characterizing each state.

\subsection{The loss of HMGBs marks the entry of senescence and coincides with chromatin changes.}

Previous studies in the context of senescence provided sparse information about the behavior of high mobility proteins and the few reports published up to date mostly focusing on HMGAs and HMGB1. We showed that both HMGBs actively decrease their nuclear levels by evicting out of the nucleus of senescence-primed cells in a sharp contrast with HMGAs that increase their expression levels upon senescence and contribute to the formation of SAHF (Narita et al., 2006). We confirmed that this eviction contributes to the senescence-associated phenotypes as we detected HMGB1 in the extracellular space acting as a SASP factor, a function previously described (Davalos et al., 2013). Thus, we focused on the nuclear events that HMGBs dictate in the proliferating cells and the changes their dynamic translocation confers during the entry of senescence, a knowledge that was still missing. To study the role of HMGBs we took advantage of the replicative exhaustion that primary cells reach and we 
confirmed that these cells drove into senescence after a finite number of population doublings in a donorand cell type-dependent manner, showing the strong idiosyncratic nature of senescence (Lecot et al., 2016). We employed RNA-seq and we showed that proliferating and senescent states are characterized by a distinct transcriptional repertoire, instructing the functional necessities of each state. Notably, direct comparison of the differential gene expression analysis acquired in the three cell types clearly demonstrated a selective up-regulation of genes involved in senescence-associated pathways (cell growth, p53 responses and ECM organization) and a robust down-regulation of genes involved in proliferating and cell cycle-associated pathways (cell-cycle regulation, replication DNA metabolism), recapitulating the existence knowledge regarding senescence (Narita et al., 2003). Interestingly, apart from the expected cell cycle associated terms, the shared down-regulation subset contained a significantly enriched group of proteins involving in genome architecture (DNA conformation, chromatin organization, and DNA packaging). The presence of this sub-group in the dataset gained our attention, as it implies that the genomic organization follows the general adaptation of the cellular homeostasis during the cellular senescence. $H M G B 1 / 2$ were among the most highly affected downregulated genes and the fact that these proteins are highly abundant in the nucleus of proliferating cells to control the transcriptional output and the genome folding, rendered them interesting candidates to focus on. Western blots and immuno-fluorescence studies determined that their protein levels significantly decrease from the majority of the nuclei linearly as the cells losing their proliferating capacity, in a sharp contrast with the behavior of HMGA1, which robustly increase during senescence (Narita et al., 2006). In fact, we demonstrated that the disappearance of nuclear HMGBs coincides with the emergence of previously well-described markers like LAMNB1 decrease and subsequent altered nuclear morphology, drop of the proliferation rate and increase of p21 and positive b-galactosidase staining. Finally we showed that nuclear loss of the HMGB1 is due to active secretion rather than proteolytic degradation (at least not the absolute levels), confirming the previous characterized paracrine role of HMGB1 during senescence (Davalos et al., 2013).

This dynamic change of the architectural proteins we and other groups observed is of a great importance, but whether these changes precede the initiation of the senescence-associated transcriptional profile or represent a consequence was still unclear. Since we are focusing on the early events of senescence the population was heterogeneous (albeit in favor of the senescence cells) adding more complexity to the already well-documented heterogeneity of senescent populations (HernandezSegura et al., 2017). We took advantage of this otherwise obstacle and we characterized the sequence of the molecular events governing the entry of senescence by employing single RNA-seq from IMR90's and pseudo-time course analysis of single-cell RNA-seq data and we clustered the sub-populations based on their expression profiles. Interestingly, we showed that the dynamic loss of HMGBs from the nuclei of the senescent cells precedes the emergence of key senescence markers like the loss of LMNB1 and the increase of CDKN1A. Analysis of the single-cell data formed a cluster of senescent cells characterized by the concurrent activation of SASP factors -a feature that represents a late-event of 
senescence- and the most robust decrease of HMGB2. The single-cell analysis revealed also the kinetics of the two HMGBs, showing that the HMGB2 decrease precedes the one of HMGB1, while the latter seems to coincide with the emergence of the characteristic senescent markers and the initiation of SASP. Collectively, this analysis clearly demonstrated that the presence of both HMGBs is important to control the cellular function of the proliferating cells, however their transcriptional shutdown and their active decrease of their nuclear levels is a prerequisite to the emergence of characteristic senescence-associated features.

Finally, we also stained the HMGBs-depleted nuclei with H3K9me3 and H3K27me3 to assess whether our replicative senescence cells form SAHF, but as it was previously reported the formation of SAHF is context dependent and very rare if any particularly in replicative senescent cells (Kosar et al., 2011). Nevertheless, we also observed a re-shuffling of the histone marks with a shift from facultative to constitutive heterochromatin, a feature that is reminiscent to the inhibitory nature of the SAHF (Narita et al., 2003). Taken together the decrease of the HMGBs nuclear levels represent an important early event of the entry to senescence that initiates genome architecture changes leading the gradual emergence of the senescence-associated phenotypes. It remains an open question whether the restoration of each HMGB or simultaneously could stall or delay the emergence of senescence and the associated features we observed.

\subsection{Genome-wide chromatin re-arrangements dictate the entry of cellular senescence}

Cellular senescence represents a complicated homeostatic mechanism that influences almost every aspect of the normal cellular function. Interaction profiles through $\mathrm{Hi}-\mathrm{C}$ have been generated before in oncogene-induced senescence (Chandra and Narita, 2013) and recently in deep replicative senescence (Criscione et al., 2016). As it was mentioned there are caveats in both studies as the one is performed in a non-physiological context (OIS) and the second one focuses on the "deep" replicative senescence. Here, we tried to understand the events leading to senescence in a slightly different spectrum as we focused on how genome organization changes could potentially influence the transcriptional transition from the proliferating state to the senescent one, a link that was still missing. Our transcriptomic datasets acquired from individual proliferating and senescent human donor cells clearly demonstrated that the switch from a transcriptional program comprised of proliferation-associated and cell cycle genes to a senescent one coincides with transcriptional changes to various architectural factors, suggesting a potential involvement of the genome organization towards the entry of senescence.

The increased heterochromatinization and the extensive changes in the expression levels of architectural proteins, prone us to generate the genome-wide interaction profile in our cell populations. We performed Hi-C in all the three cell types with technical and biological replicates and we efficiently 
generated the interaction maps for both the proliferating and senescent cells in a resolution as high as $20-\mathrm{kbp}$. These are the first generated interaction maps focusing on the dynamic events modifying the chromatin folding during the entry of replicative senescence. The 200-kpb-generated Hi-C maps were reproducible between the different donors of the same cell type, but they differ between the different cell types recapitulating the diversity in the epigenetic state and the intrinsic characteristics unique to each of these different cells types. Moreover, the fact that the interaction profiles differ among then, reinforces the general concept that senescence is strongly cell- and context- dependent (Lecot et al., 2016). Nevertheless, despite the apparent cell type specific changes, our generated Hi-C maps possess shared properties between the different cell types and contain all the features of the high-order structure, allowing us to generalize some of our conclusions and link them with the entry of cellular senescence.

The segregation of the high-order structure in A and B compartments is usually associated with the transcriptional competency of the genomic regions (Lieberman-aiden et al., 2009). Interestingly and in contrast to the study of Criscione (Criscione et al., 2016), we observed a minor switch of regions between $\mathrm{A}$ and $\mathrm{B}$ compartments, a finding that is quite surprising considering the transcriptional switch and the re-shuffling of the histone marks. A closer inspection to the study of Criscione (Criscione et al., 2016) reveals that despite the extensive compartment switching, genes associated with senescence represent only a small subset (from 8 to $14 \%$ ) switches from A to B and an even smaller fraction (1 to 4\%) from B to A compartments. Thus, it seems that genes associated with senescence are poised to a transcriptional-primed state and other than compartment switch is the main trigger for activation. Despite the lack of compartment switch, we did observe changes in the high-order structure, especially when we assess the inter- and intra-chromosomal interactions. The analysis of the inter-chromosomal interaction revealed that all the three cell types displayed decreased cell-type specific interactions indicating a less-mobile chromatin arrangement in the nuclear space. This in line with the observations of the oncogene-induced senescence (Chandra and Narita, 2013) but differ from the "deep" replicate senescence of Criscione (Criscione et al., 2016). The intra-chromosomal interactions also support the most compacted chromatin arrangement during replicative senescence as we showed the emergence of stronger long-range interactions and stronger insulation between higher-order domains. Both inter- and intra-chromosomal interaction-profiles are in line with the increase of constitutive heterochromatin and the acquired compacted structure of the chromatin during senescence. In fact, the observed loss of LMNB1 in our senescent cells might explain the strict structure of the senescent chromatin and resemble the model Chandra proposed during oncogene-induced senescence (Chandra et al., 2015). Additionally, this restricted configuration possibly instructs the transcriptional switch-off of the proliferation genes and a general stricter control of the transcriptional output similarly to SAHF formation (Narita et al., 2003).

Having describe the general view of the chromatin folding we sought to assess the organization of the genome in TAD level. As it was mentioned before, TADs generally preserve their structure during 
changes in the cellular homeostasis even in extreme examples as the differentiation of stem cells to somatic (Dixon et al., 2015). Using our generated Hi-C maps and the "TADtool" software (Kruse et al., 2016) we showed that entry of senescence is accompanied by extensive re-arrangement of TADs margins genome-wide (mainly shifting the boundary and fusing neighboring TADs while the separation of TADs was less pronounced). The resulted almost 50\% in IMR90's and MSCs, and the impressive $70 \%$ altered TADs in HUVECs pinpoints the strong link between chromatin organization and the induction of senescence. Interestingly, this link is not only restricted to the structural organization, but also strongly attributes to the establishment of the transcriptional profile characterizing the proliferating or the senescent state. "Topologically-intrinsic lexicographic ordering" or TiLO (Johnson, 2014) clearly demonstrated the spatial clustering of functionally related TADs as the number and the identity of TADs comprising each cluster was highly correlated either the proliferating or the senescent state and the signature pathways each of these states are linked to. In fact, considering the absence of compartment switch in our replicative senescence model, it seems that the coordinated clustering of the TADs is the driving force of the expression of genes related to senescence. This is of a great importance because it underscores TADs not only as a self-organizing structure but also, as a functional entity capable to dynamically cluster in the 3D space and orchestrate the transcriptional repertoire.

\subsection{The nuclear loss of HMGB2 instructs the senescence associate transcriptional activity and induces rapid genomic re-organization}

High mobility group proteins are very abundant proteins in the nucleus of all our three different cells types. During senescence, both HMGB1 and HMGB2 decrease their nuclear protein levels and robustly switch off their transcriptional activity. Up to date, the function of HMGBs in the nucleus was focused on their role as architectural proteins in the context of transcriptional enhancement (Ueda and Yoshida, 2010). Their actual contribution in the genomic organization and the changes their dynamic eviction results to was still elusive. Although the loss of HMGBs from the nucleus and the role of HMGB1 as a SASP factor upon senescence was shown before, the consequences of their absence in the nucleus and how this contributes to the senescence state was still not described. Here, we provide a very comprehensive description of their nuclear role as architectural proteins by identifying their binding sites genome-wide circumventing their incompatibility with standard fixative methods (Teves et al., 2016) and the hit-and-run binding fashion (Štros, 2010) by using a tailored chip protocol.

Despite the aforementioned limitations, we managed to identify more than 1574 and 1188 HMGB2 binding sites in proliferating HUVECs and IMR90s respectively, and 391 in senescent IMR90's. Although the number of the peaks does not match their actual high abundancy in the nucleus of proliferating cells, it is still sufficient to provide the binding profile of these proteins and their associated transcriptional pathways involved. Interestingly, HMGB2's binding sites in proliferating 
cells reside in promoters and gene-bodies and when we searched of the relative GO terms of the surrounding genes both the up-and the down-regulated genes were enriched with senescence-associated pathways. The role of HMGB2 in proliferating cells is not only restricted in the regulation of the transcriptional landscape as we showed the implication of HMGB2 in the genomic organization. Namely a significant subset of the HMGB2 peaks resides at TAD boundaries contributing in the demarcation of TADs complementing the well-characterized role of CTCF (Ong and Corces, 2014). Similarly to Monte et al (Monte et al., 2016), our findings suggest that certain genomic sites could be occupied of either HMGB2 or CTCF but we showed that the is not mediated through direct interaction between the two proteins. Nevertheless, the substantial HMGB2 peaks at the TAD boundaries suggest HGMB2 as a potent contributor in the establishment of the genome organization especially considering the persistent binding of HMGBs during mitosis (Pallier C, Scaffidi, Chopineau-Proust S, Agresti A, Nordmann P, Bianchi M, 2003).

The binding profile of HMGB2 and the interplay with CTCF especially at the TAD boundaries seems to instruct the genomic organization upon the entry of senescence. Intriguingly, we showed that the occupancy of HMGB2 in both sides of the TAD or the reciprocal occupancy with HMGB2 on the one and CTCF on the other side dictate the integrity of the TADs during the entry of senescence. Namely, we clearly demonstrated that the demarcation of TADs by HMGB2 on both sides is sufficient to preserve the TAD's integrity upon senescence-entry, whereas demarcation of TADs by HMGB2 and CTCF in the two side of the TADs preferentially lead to shift of the boundary in senescent cells. Taking into account that the majority of the HMGB2-bound genes are up-regulated upon the entry of senescence and the absence of A and B compartment switch, we could deduce that the HGMB2 translocation and the resulting changes in the TADs integrity they are bound to, are the main drivers of the emergence of senescence and the accompanied transcriptional switch. Especially the fact that the majority of the HMGB2 bound genes consist of up-regulated upon the entry of senescence and plenty of them are associated with relevant to senescence pathways, reinforces the interplay between HMGB2 nuclear loss, chromatin organization and transcriptional switch upon senescence. Interestingly despite the general decrease of the protein levels, we could still identify sites of persistent HMGB2 binding in senescent cells, however none of these peaks are overlapping with any SASP factor arguing with the findings observed for OIS (Aird et al., 2016). Thus, we conclude that the contribution of HMGB2 in SASP is not related to a direct binding on SASP genes and possibly the expression of genes represent a downstream target of the transcriptional alterations or the chromatin re-arrangements controlled by HMGB2.

As it was mentioned before, the transcriptional shutdown of HMGBs and the re-arrangement of the genomic structure precede the increase of senescence markers implying the importance of this intermediate step. To gain further insight we efficiently performed HMGB2 knockdown taking advantage of the self-delivering siRNAs and we focused on changes of the architectural proteins. Super resolution and fluorescent microscopy demonstrated the crosstalk between CTCF and HMGB2, as cells 
depleted of HMGB2-nuclear signaling promote the formation of discrete CTCF foci. This robust clustering of the CTCF, reminiscent of the SAHF produced in oncogene-induced senescence (Narita et al., 2003), was not a result of altered levels of binding on the pre-existing CTCF sites of formation of "neoCTCF" binding sites, recapitulating the mutual exclusive binding profile of the two proteins seen in ChIP-seq and underlining the dynamic genomic re-arrangements upon the loss of HMGB2. Importantly we repeated the double CTCF and HMGB2 stainings in our senescent cells and we clearly demonstrated a dynamic CTCF clustering in the HMGB2-depleted nuclei of senescent cells, conferring the CTCF clustering as a distinct feature of senescence. We went a step forward by applying Hi-ChIP, targeting the interacting regions bound by $\mathrm{CTCF}$ and we proved the loss of HMGB2-binding suffices to bring sites bound by CTCF in close proximity genomic and promote the clustering. Therefore, the formation of these senescence-associated CTCF clustering (SICC) results due to the loss of the HMGB2mediated local insulation on adjacent CTCF loops and highlights the pivotal role of HMGB2 in the genomic architecture. Finally, we demonstrated that the CTCF clustering is directly mediated by HMGB2 loss and not by a downstream pathway, but re-introducing the HMGB2 in the senescent cells and decreasing the formation of CTCF foci. Strikingly, the fact that we were not able to completely deplete the formation of CTCF or reverse them in later stages of senescence suggests that the formation of these foci is an important feature of the senescent cells and a powerful mediator of senescence maintenance.

The contribution of HMGB2 to the SICC formation suggested that the absence of HMGB2 from the nucleus of senescent cells is one of the main drivers of the chromatin alterations and that it could mimic some of the features accompanying senescence. Indeed, using the self-delivering siRNAs we efficiently knock-down HMGB2 and generated the Hi-maps and we observe that the absence of nuclear HMGB2 recapitulates the results of Hi-C, highlighting that HMGB2 is the upstream effector molecule for the architectural changes seen. Moreover, the absence of HMGB2 promotes the heterochromatinization seen in Hi-C mediated by the increase of HP1a and the decrease of H3K27me3. Surprisingly despite the fact that the HMGB2 knockdown affected the genomic organization it was not sufficient to drive genes-specific transcriptional alterations but rather an overall reduction of the nascentRNA production. The latter finding in combination to the absence of common senescence markers (Bgalactosidase assay, cell cycle arrest) might propose that the short-term nature of the knockdown represent a technical obstacle to recapitulate the complete senescence-associated repertoire. Nevertheless, HMGB2 similarly to HMGA1 is responsible to guide the formation of high-order structure in the nucleus of senescent cells albeit it does it by decreasing its nuclear levels in sharp contrast with the increased presence of HMGA1. The model describing the impact of HMGB2 upon the entry to senescence and the crosstalk with CTCF is depicted in Figure 4.1. 


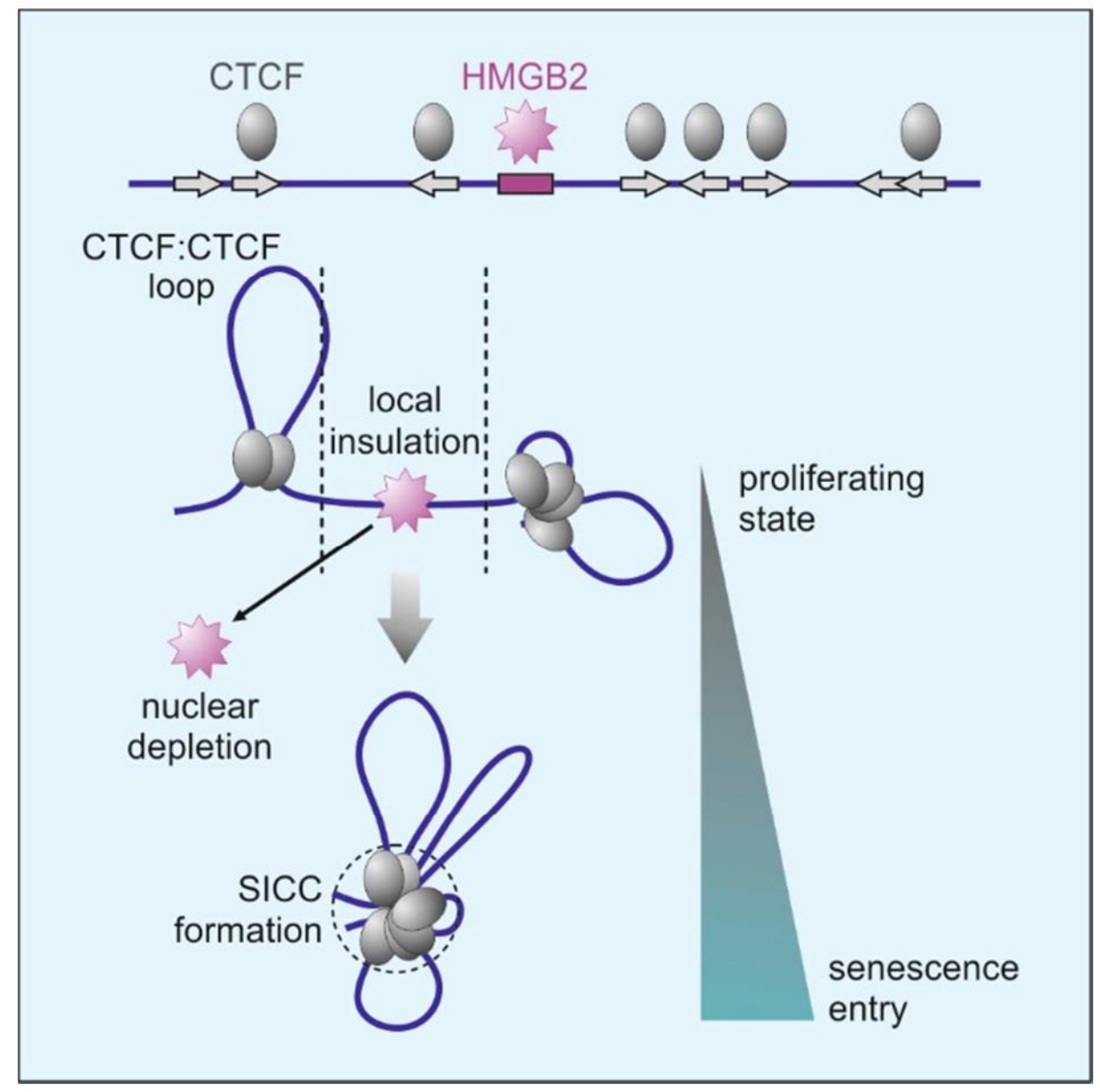

Figure 4.1 Organization of senescence chromatin in SICC

In young cells HMGB2 binds chromatin and insulates neighboring CTCF-mediated loops. This insulation is lost as HMGB2 decreases upon the entry of senescence resulting to CTCF clustering.

\subsection{HMGB1 complements HMGB1 to TAD demarcation but its nuclear loss is associated with distinct to HMGB1 genomic re-arrangements}

The high sequence similarity of HMGB1 and HMGB2 could also reflect similar mode of action and functional redundancy. The trend they follow upon senescence also reflects some similarity as they both switch off their expression levels and dynamically decrease their nuclear protein levels. On the contrary, it seems that only HMGB1 translocates out of the nucleus and increases its levels in the culture medium where it acts as a SASP factor. To compare the ab nucleo function of HMGBs we performed the tailored Chip-seq protocol similarly to HMGB2. The HMGB1 peaks were associated with gene promoters, active genes and $\mathrm{H} 3 \mathrm{~K} 27$ ac-bound regions reinforcing its characterized role as transcriptional activator. Interestingly, the resulted genes do not contain any of the well-documented SASP factor suggesting that the contribution of HMGB1 to SASP is not mediated through direct binding on these genes. On the other hand, a subset of these peaks is associated with the NOTCH/TGFb signaling cascade which represents the second wave of the paracrine signaling (Acosta et al., 2013; Hoare et al., 2016). 
It is worth noted, that the peaks of the two HMGBs are rarely overlapping suggesting a clear distinction of the pathways each protein controls. Similar to HMGB2, 21\% of the HMGB1 resides in boundaries demarcating the TAD, but in sharp to HMGB2 contrast, these TADs are devoid of CTCF. Furthermore, we showed that this demarcation is lost upon the entry of senescence as we observed an obvious loss of the TADs insulation following the loss of the nuclear HMGB1. Although both HMGB1 and CTCF demarcate the TADs and their loss results in architectural changes, the impact on TADs upon the entry of senescence is different between the two proteins, as in the case of HMGB1-demarcated TADs either shift their boundary or collapse into a larger TAD and these alterations are irrespective of CTCF binding. Taken together, while the mode of binding is similar, HMGBs differentiate their binding targets and the demarcation of TADs in respect to CTCF. The latter is convincingly shown by the fact that the knockdown of HMGB1 does not suffice for the formation of CTCF in sharp contrast with HMGB2. On the other hand, HMGB1 largely associates with upregulation of genes with GO terms associated with SASP (IL4 and IL13 signaling pathway, positive regulation of secretion, IL17 signaling pathway). This finding underlines more the observation that HMGB1 leaves the nucleus with slower kinetics than HMGB2 and involves in the induction of SASP, a cascade that also emerges later during senescence. It also supports the results of the single-analysis drawing the sequence of the molecular events towards the entry of senescence.

Taken all together, we were able to identify the actual sequence of the events that govern the induction of senescence. Initially gradual loss of HMGB2 induces chromatin re-arrangements with the CTCF clustering representing a prominent feature possibly for the strict maintenance of the compacted and replicative-incompatible senescence state. HMGB1 represents a later event of the senescence entry and the first wave of the HMGB2-based architectural changes, reinforcing the action of HMGB2 as a second wave of architectural changes in order to re-organize the 3D genome architecture of the senescent cells. Finally, yet importantly, the loss of HMGB1 marks the initiation of active secretion of the SASP factor to impact on the neighboring cells.

\subsection{HMGB1 is a bona fide RNA-binding protein regulating the nascent-RNA turnover of SASP factors}

As it was mentioned before, our main focus regarding HMGBs was centered in their role as transcription factors. The identification of the protein factors associated with HMGBs through coimmunoprecipitation coupled with mass-spectrometry led us to characterize a broad spectrum of RBPs associate with HMGBs, capable of regulating the nascent RNA turnover. Interestingly, the overlap of the immunoprecipitated factors was 25\% for HMGB1 (46 out of 182) and 32\% for HMGB2 (46 out of 143). These high percentages drops considerably when we exclude the RBPs described by the work of Castello (Castello et al., 2016) to 9\% and 11\% for HMGB1 and HMGB2 respectively. The very high 
occupancy of RBPs in the shared proteins implies that at least there is a functional redundancy between the two proteins regarding their role in the nascent-RNA regulation in sharp contrast with their architectural role and their roles outside of the eukaryotic nucleus. Collectively, since all the aforementioned findings combined with the results of Castello attributing HMGBs an RNA-binding capacity (Castello et al., 2016), let us to characterize HMGBs as bona fide RNA binding proteins. The RNA binding capacity of HMGBs is of a great importance as it adds another layer of the ab nucleo roles of these proteins and the induction of the senescence transcriptional program but also increases the complexity to describe their contribution in the senescence phenotype.

To convincingly show the RNA-binding functionality of HMGBs we performed Clip and we identified various RNA targets bound by HMGBs. The ability of HMGBs to bind and regulate RNA targets is of a great importance especially in the context of senescence as it describes an extra layer of regulation, which was unprecedented up until now. The two proteins share a significant number of RNA binding targets suggesting some functional redundancy as RNA regulators. Interestingly the shared targets are closely associated with the senescence profile and specifically with the induction of SASP as many of bound genes are inflammatory targets. The ability of HMGB1 to control the SASP at the RNA level adds another layer to the already complex feedback loop encompassing the cell cycle arrest, chromatin architecture, nascent mRNA production and the secretion of SASP factors. We sought to gain further insight in the regulation of nascent RNA production of SASP factors from HMGB1 and we focused on the RNA-binding protein ILF3 an important regulator of the expression of SASP genes (Tominaga-Yamanaka et al., 2012). Mass-spectrometry identify ILF3 as a direct HMGB1 protein partner and Clip showed that ILF3 RNA is bound by HMGB1. Furthermore ILF3 was part of the downregulated genes upon both HMGB1-knockdown and replicate senescence following the behavior of HMGBs upon the entry of senescence.

Taken together we showed that HMGBs are capable of RNA binding, regulating the entry of senescence trough the nascent-RNA production. This finding adds another layer of this multifaceted role of HMGB1 in the induction of senescence and in particular, the regulation and expansion of SASP. 


\section{References}

Acosta, J.C., O’Loghlen, A., Banito, A., Guijarro, M. V., Augert, A., Raguz, S., Fumagalli, M., Da Costa, M., Brown, C., Popov, N., et al. (2008). Chemokine Signaling via the CXCR2 Receptor Reinforces Senescence. Cell 133, 1006-1018.

Acosta, J.C., Banito, A., Wuestefeld, T., Georgilis, A., Janich, P., Morton, J.P., Athineos, D., Kang, T.W., Lasitschka, F., Andrulis, M., et al. (2013). A complex secretory program orchestrated by the inflammasome controls paracrine senescence. Nat. Cell Biol. 15, 978990.

Afshari, C.A., Nichols, M.A., Xiong, Y., and Mudryj, M. (1996). A role for a p21-E2F interaction during senescence arrest of normal human fibroblasts. Cell Growth Differ. 7, 979988.

Agger, K., Agger, K., Cloos, P. a C., Cloos, P. a C., Rudkj, L., Rudkj, L., Williams, K., Williams, K., Andersen, G., Andersen, G., et al. (2009). Karl Agger, Paul A.C. Cloos, Lise Rudkjær, Kristine Williams, Gitte Andersen, Jesper Christensen, and Kristian Helin 1. Genes Dev. 1171-1176.

Aird, K.M., Iwasaki, O., Kossenkov, A. V., Tanizawa, H., Fatkhutdinov, N., Bitler, B.G., Le, L., Alicea, G., Yang, T.L., Johnson, F.B., et al. (2016). HMGB2 orchestrates the chromatin landscape of senescence-associated secretory phenotype gene loci. J. Cell Biol. 215, 325-334. Anderson, E., Peluso, S., Lettice, L.A., and Hill, R.E. (2012). Human limb abnormalities caused by disruption of hedgehog signaling. Trends Genet. 28, 364-373.

Baker, D.J., Perez-Terzic, C., Jin, F., Pitel, K., Niederländer, N.J., Jeganathan, K., Yamada, S., Reyes, S., Rowe, L., Hiddinga, H.J., et al. (2008). Opposing roles for p16Ink4a and p19Arf in senescence and ageing caused by BubR1 insufficiency. Nat. Cell Biol. 10, 825836.

Baker, D.J., Wijshake, T., Tchkonia, T., Lebrasseur, N.K., Childs, B.G., Van De Sluis, B., Kirkland, J.L., and Van Deursen, J.M. (2011). Clearance of p16 Ink4a-positive senescent cells delays ageing-associated disorders. Nature 479, 232-236.

Barradas, M., Anderton, E., Acosta, J.C., Li, S. De, Banito, A., Rodriguez-Niedenführ, M., Maertens, G., Banck, M., Zhou, M.M., Walsh, M.J., et al. (2009). Histone demethylase JMJD3 contributes to epigenetic control of INK4a/ARF by oncogenic RAS. Genes Dev. 23, 1177-1182. 
Beach, D., Serrano, M., and Hannon, G.J. (1993). inhibition of cyclin D / CDK4. 366, 704707.

Bennett, M.R., Macdonald, K., Chan, S., Boyle, J.J., and Weissberg, P.L. (1998).

Proliferation, Cell Senescence, and Apoptosis in Human. 704-712.

Bianchi, M.E., and Agresti, A. (2005). HMG proteins: Dynamic players in gene regulation and differentiation. Curr. Opin. Genet. Dev. 15, 496-506.

Calogero, S., Grassi, F., Aguzzi, A., Voigtländer, T., Ferrier, P., Ferrari, S., and Bianchi, M.E. (1999). The lack of chromosomal protein Hmg1 does not disrupt cell growth but causes lethal hypoglycaemia in newborn mice. Nat. Genet. 22, 276-280.

Campisi, J. (2013). Aging, Cellular Senescence, and Cancer. Annu. Rev. Physiol. 75, 685705.

Campos, E.I., Stafford, J.M., and Reinberg, D. (2014). Epigenetic inheritance: Histone bookmarks across generations. Trends Cell Biol. 24, 664-674.

Castello, A., Fischer, B., Frese, C.K., Horos, R., Alleaume, A.M., Foehr, S., Curk, T., Krijgsveld, J., and Hentze, M.W. (2016). Comprehensive Identification of RNA-Binding Domains in Human Cells. Mol. Cell 63, 696-710.

Catena, R., Escoffier, E., Caron, C., Khochbin, S., Martianov, I., and Davidson, I. (2009). HMGB4, a Novel Member of the HMGB Family, Is Preferentially Expressed in the Mouse Testis and Localizes to the Basal Pole of Elongating Spermatids1. Biol. Reprod. 80, 358-366.

Cesare, A.J., and Karlseder, J. (2012). A three-state model of telomere control over human proliferative boundaries. Curr. Opin. Cell Biol. 24, 731-738.

Chandra, T., and Narita, M. (2013). High-order chromatin structure and the epigenome in SAHFs. Nucl. (United States) 4, 1-6.

Chandra, T., Kirschner, K., Thuret, J.Y., Pope, B.D., Ryba, T., Newman, S., Ahmed, K., Samarajiwa, S.A., Salama, R., Carroll, T., et al. (2012). Independence of Repressive Histone Marks and Chromatin Compaction during Senescent Heterochromatic Layer Formation. Mol. Cell 47, 203-214.

Chandra, T., Ewels, P.A., Schoenfelder, S., Furlan-Magaril, M., Wingett, S.W., Kirschner, K., Thuret, J.Y., Andrews, S., Fraser, P., and Reik, W. (2015). Global reorganization of the nuclear landscape in senescent cells. Cell Rep. 10, 471-483.

Chang, J., Wang, Y., Shao, L., Laberge, R.M., Demaria, M., Campisi, J., Janakiraman, K., Sharpless, N.E., Ding, S., Feng, W., et al. (2016). Clearance of senescent cells by ABT263 


\section{References}

rejuvenates aged hematopoietic stem cells in mice. Nat. Med. 22, 78-83.

Chiche, A., Le Roux, I., von Joest, M., Sakai, H., Aguín, S.B., Cazin, C., Salam, R., Fiette, L., Alegria, O., Flamant, P., et al. (2017). Injury-Induced Senescence Enables In Vivo

Reprogramming in Skeletal Muscle. Cell Stem Cell 20, 407-414.e4.

Childs, B.G., Durik, M., Baker, D.J., and Van Deursen, J.M. (2015). Cellular senescence in aging and age-related disease: From mechanisms to therapy. Nat. Med. 21, 1424-1435.

Cho, W., Koo, J.Y., Park, Y., Oh, K., Lee, S., Song, J.S., Bae, M.A., Lim, D., Lee, D.S., and Park, S.B. (2017). Treatment of Sepsis Pathogenesis with High Mobility Group Box Protein 1-Regulating Anti-inflammatory Agents. J. Med. Chem. 60, 170-179.

Coppé, J.-P., Desprez, P.-Y., Krtolica, A., and Campisi, J. (2010). The Senescence-Associated Secretory Phenotype: The Dark Side of Tumor Suppression. Annu. Rev. Pathol. Mech. Dis. 5, 99-118.

Coppé, J.P., Patil, C.K., Rodier, F., Sun, Y., Muñoz, D.P., Goldstein, J., Nelson, P.S., Desprez, P.Y., and Campisi, J. (2008). Senescence-associated secretory phenotypes reveal cell-nonautonomous functions of oncogenic RAS and the p53 tumor suppressor. PLoS Biol. 6.

Criscione, S.W., Cecco, M. De, Siranosian, B., Zhang, Y., Kreiling, J.A., Sedivy, J.M., and Neretti, N. (2016). Biomolecules: Reorganization of chromosome architecture in replicative cellular senescence. Sci. Adv. 2.

D'Adda Di Fagagna, F. (2008). Living on a break: Cellular senescence as a DNA-damage response. Nat. Rev. Cancer 8, 512-522.

D’Adda Di Fagagna, F., Reaper, P.M., Clay-Farrace, L., Fiegler, H., Carr, P., Von Zglinicki, T., Saretzki, G., Carter, N.P., and Jackson, S.P. (2003). A DNA damage checkpoint response in telomere-initiated senescence. Nature 426, 194-198.

Datto, M.B., Li, Y., Panus, J.F., Howe, D.J., Xiong, Y., and Wang, X.F. (1995). Transforming growth factor $\beta$ induces the cyclin-dependent kinase inhibitor p21 through a p53-independent mechanism. Proc. Natl. Acad. Sci. U. S. A. 92, 5545-5549.

Davalli, P., Mitic, T., Caporali, A., Lauriola, A., and D'Arca, D. (2016). ROS, Cell Senescence, and Novel Molecular Mechanisms in Aging and Age-Related Diseases. Oxid. Med. Cell. Longev. 2016.

Davalos, A.R., Kawahara, M., Malhotra, G.K., Schaum, N., Huang, J., Ved, U., Beausejour, C.M., Coppe, J.P., Rodoer, F., and Campisi, J. (2013). p53-dependent release of Alarmin 


\section{References}

HMGB1 is a central mediator of senescent phenotypes. J. Cell Biol. 201, 613-629.

Dechat, T., Pfleghaar, K., Sengupta, K., Shimi, T., Shumaker, D.K., Solimando, L., and Goldman, R.D. (2008). Nuclear lamins: Major factors in the structural organization and function of the nucleus and chromatin. Genes Dev. 22, 832-853.

Dekker, J., Rippe, K., Dekker, M., and Kleckner, N. (2002). Capturing chromosome conformation. Science (80-. ). 295, 1306-1311.

van Delft, M.F., Wei, A.H., Mason, K.D., Vandenberg, C.J., Chen, L., Czabotar, P.E., Willis, S.N., Scott, C.L., Day, C.L., Cory, S., et al. (2006). The BH3 mimetic ABT-737 targets selective Bcl-2 proteins and efficiently induces apoptosis via Bak/Bax if Mcl-1 is neutralized. Cancer Cell 10, 389-399.

Demaria, M., Ohtani, N., Youssef, S.A., Rodier, F., Toussaint, W., Mitchell, J.R., Laberge, R.M., Vijg, J., VanSteeg, H., Dollé, M.E.T., et al. (2014). An essential role for senescent cells in optimal wound healing through secretion of PDGF-AA. Dev. Cell 31, 722-733.

Denker, A., and De Laat, W. (2016). The second decade of 3C technologies: Detailed insights into nuclear organization. Genes Dev. 30, 1357-1382.

Despang, A., Schöpflin, R., Franke, M., Ali, S., Jerković, I., Paliou, C., Chan, W.L., Timmermann, B., Wittler, L., Vingron, M., et al. (2019). Functional dissection of the Sox9Kcnj2 locus identifies nonessential and instructive roles of TAD architecture. Nat. Genet. 51, $1263-1271$.

van Deursen, J.M. (2019). Senolytic therapies for healthy longevity. Science (80-. ). 364, 636-637.

Dimri, G.P., Lee, X., Basile, G., Acosta, M., Scott, G., Roskelley, C., Medrano, E.E., Linskens, M., Rubelj, I., Pereira-Smith, O., et al. (1995). A biomarker that identifies senescent human cells in culture and in aging skin in vivo. Proc. Natl. Acad. Sci. U. S. A. 92, 9363-9367.

Dimri, G.P., Nakanishi, M., Desprez, P.Y., Smith, J.R., and Campisi, J. (1996). Inhibition of E2F activity by the cyclin-dependent protein kinase inhibitor p21 in cells expressing or lacking a functional retinoblastoma protein. Mol. Cell. Biol. 16, 2987-2997.

Dixon, J.R., Selvaraj, S., Yue, F., Kim, A., Li, Y., Shen, Y., Hu, M., Liu, J.S., and Ren, B. (2012). Topological domains in mammalian genomes identified by analysis of chromatin interactions. Nature 485, 376-380.

Dixon, J.R., Jung, I., Selvaraj, S., Shen, Y., Antosiewicz-Bourget, J.E., Lee, A.Y., Ye, Z., 
Kim, A., Rajagopal, N., Xie, W., et al. (2015). Chromatin architecture reorganization during stem cell differentiation. Nature 518, 331-336.

Ellerman, J.E., Brown, C.K., De Vera, M., Zeh, H.J., Billiar, T., Rubartelli, A., and Lotze, M.T. (2007). Masquerader: High mobility group box-1 and cancer. Clin. Cancer Res. 13, 2836-2848.

Eriksson, M., Brown, W.T., Gordon, L.B., Glynn, M.W., Singer, J., Scott, L., Erdos, M.R., Robbins, C.M., Moses, T.Y., Berglund, P., et al. (2003). Recurrent de novo point mutations in lamin A cause Hutchinson-Gilford progeria syndrome. Nature 423, 293-298.

Evangelou, K., Lougiakis, N., Rizou, S. V., Kotsinas, A., Kletsas, D., Muñoz-Espín, D., Kastrinakis, N.G., Pouli, N., Marakos, P., Townsend, P., et al. (2017). Robust, universal biomarker assay to detect senescent cells in biological specimens. Aging Cell 16, 192-197.

Fabre, P.J., Leleu, M., Mormann, B.H., Lopez-Delisle, L., Noordermeer, D., Beccari, L., and Duboule, D. (2017). Large scale genomic reorganization of topological domains at the HoxD locus. Genome Biol. 18, 1-15.

Festuccia, N., Gonzalez, I., Owens, N., and Navarro, P. (2017). Mitotic bookmarking in development and stem cells. Dev. 144, 3633-3645.

Freund, A., Laberge, R.M., Demaria, M., and Campisi, J. (2012). Lamin B1 loss is a senescence-associated biomarker. Mol. Biol. Cell 23, 2066-2075.

Ghavi-Helm, Y., Jankowski, A., Meiers, S., Viales, R.R., Korbel, J.O., and Furlong, E.E.M. (2019). Highly rearranged chromosomes reveal uncoupling between genome topology and gene expression. Nat. Genet. 51, 1272-1282.

Gibcus, J.H., Samejima, K., Goloborodko, A., Samejima, I., Naumova, N., Nuebler, J., Kanemaki, M.T., Xie, L., Paulson, J.R., Earnshaw, W.C., et al. (2018). A pathway for mitotic chromosome formation. Science (80-. ). 359.

Goodwin, G.H., Sanders, C., Johns, E.W., and Electrophoresis, P. (2000). GH_et_al-1973European_Journal_of_Biochemistry. 19,14-19.

Guelen, L., Pagie, L., Brasset, E., Meuleman, W., Faza, M.B., Talhout, W., Eussen, B.H., De Klein, A., Wessels, L., De Laat, W., et al. (2008). Domain organization of human chromosomes revealed by mapping of nuclear lamina interactions. Nature 453, 948-951.

Guo, Y., Xu, Q., Canzio, D., Shou, J., Li, J., Gorkin, D.U., Jung, I., Wu, H., Zhai, Y., Tang, Y., et al. (2015). CRISPR Inversion of CTCF Sites Alters Genome Topology and Enhancer/Promoter Function. Cell 162, 900-910. 
Harbour, J.W., and Dean, D.C. (2000). The Rb/E2F pathway: Expanding roles and emerging paradigms. Genes Dev. 14, 2393-2409.

Harmston, N., Ing-Simmons, E., Tan, G., Perry, M., Merkenschlager, M., and Lenhard, B. (2017). Topologically associating domains are ancient features that coincide with Metazoan clusters of extreme noncoding conservation. Nat. Commun. 8 .

Hergeth, S.P., and Schneider, R. (2015). \&quot;Histones and Chromatin\&quot; Review series The H1 linker histones: multifunctional proteins beyond the nucleosomal core particle. 16 , 1439-1453.

Hernandez-Segura, A., de Jong, T. V., Melov, S., Guryev, V., Campisi, J., and Demaria, M. (2017). Unmasking Transcriptional Heterogeneity in Senescent Cells. Curr. Biol. 27, 26522660.e4.

Hoare, M., Ito, Y., Kang, T.W., Weekes, M.P., Matheson, N.J., Patten, D.A., Shetty, S., Parry, A.J., Menon, S., Salama, R., et al. (2016). NOTCH1 mediates a switch between two distinct secretomes during senescence. Nat. Cell Biol. 18, 979-992.

Jackson, S.P., Samson, L.D., Estep, P., Church, G.M., Samson, L.D., Rosenbach, A.S., Ideker, T., Samson, L.D., Rosenbach, A.S., Ideker, T., et al. (2006). Lamin A - Dependent Nuclear. 312, 1059-1064.

Jarrard, D.F., Sarkar, S., Shi, Y., Yeager, T.R., Magrane, G., Kinoshita, H., Nassif, N., Meisner, L., Newton, M.A., Waldman, F.M., et al. (1999). p16/pRb pathway alterations are required for bypassing senescence in human prostate epithelial cells. Cancer Res. 59, $2957-$ 2964.

Jeon, O.H., Kim, C., Laberge, R.M., Demaria, M., Rathod, S., Vasserot, A.P., Chung, J.W., Kim, D.H., Poon, Y., David, N., et al. (2017). Local clearance of senescent cells attenuates the development of post-traumatic osteoarthritis and creates a pro-regenerative environment. Nat. Med. 23, 775-781.

Johnson, J. (2014). Topological graph clustering with thin position. Geom. Dedicata 169, $165-173$.

Jun, J. Il, and Lau, L.F. (2010). The matricellular protein CCN1 induces fibroblast senescence and restricts fibrosis in cutaneous wound healing. Nat. Cell Biol. 12, 676-685.

Kang, R., Livesey, K.M., Zeh, H.J., Lotze, M.T., and Tang, D. (2010). HMGB1: A novel Beclin 1-binding protein active in autophagy. Autophagy 6, 1209-1211.

Khleif, S.N., Degregori, J., Yee, C.L., Otterson, G.A., Kaye, F.J., Nevins, J.R., and Howley, 
P.M. (1996). Inhibition of cyclin D-CDK4/CDK6 activity is associated with an E2F-mediated induction of cyclin kinase inhibitor activity. Proc. Natl. Acad. Sci. U. S. A. 93, 4350-4354.

Kim, K.-H., Chen, C.-C., Monzon, R.I., and Lau, L.F. (2013). Matricellular Protein CCN1 Promotes Regression of Liver Fibrosis through Induction of Cellular Senescence in Hepatic Myofibroblasts. Mol. Cell. Biol. 33, 2078-2090.

Kim, Y.H., Kwak, M.S., Shin, J.M., Hayuningtyas, R.A., Choi, J.E., and Shin, J.S. (2018). Inflachromene inhibits autophagy through modulation of Beclin 1 activity. J. Cell Sci. 131.

Koch, C.M., Joussen, S., Schellenberg, A., Lin, Q., Zenke, M., and Wagner, W. (2012).

Monitoring of cellular senescence by DNA-methylation at specific CpG sites. Aging Cell 11, 366-369.

Kosar, M., Bartkova, J., Hubackova, S., Hodny, Z., Lukas, J., and Bartek, J. (2011).

Senescence-associated heterochromatin foci are dispensable for cellular senescence, occur in a cell type- And insult-dependent manner, and follow expression of p16ink4a. Cell Cycle 10, $457-468$.

Krefting, J., Andrade-Navarro, M.A., and Ibn-Salem, J. (2018). Evolutionary stability of topologically associating domains is associated with conserved gene regulation. BMC Biol. $16,87$.

Krijger, P.H.L., and De Laat, W. (2016). Regulation of disease-associated gene expression in the 3D genome. Nat. Rev. Mol. Cell Biol. 17, 771-782.

Kruse, K., Hug, C.B., Hernández-Rodríguez, B., and Vaquerizas, J.M. (2016). TADtool: Visual parameter identification for TAD-calling algorithms. Bioinformatics 32, 3190-3192. Krynetskaia, N., Xie, H., Vucetic, S., Obradovic, Z., and Krynetskiy, E. (2008). High mobility group protein B1 is an activator of apoptotic response to antimetabolite drugs. Mol. Pharmacol. 73, 260-269.

Kubo, N., Ishii, H., Gorkin, D., Meitinger, F., Xiong, X., Fang, R., Liu, T., Ye, Z., Li, B., Dixon, J., et al. (2017). Preservation of Chromatin Organization after Acute Loss of CTCF in Mouse Embryonic Stem Cells. Doi.Org 118737.

Kuilman, T., Michaloglou, C., Vredeveld, L.C.W., Douma, S., van Doorn, R., Desmet, C.J., Aarden, L.A., Mooi, W.J., and Peeper, D.S. (2008). Oncogene-Induced Senescence Relayed by an Interleukin-Dependent Inflammatory Network. Cell 133, 1019-1031.

L. HAYFLICK, P.S.M., and Wistnr (1961). the Serial Cultivation of Human Diploid Cell Strains. Exp. Cell Res. 1. 
Laugsch, M., Bartusel, M., Rehimi, R., Alirzayeva, H., Karaolidou, A., Crispatzu, G., Zentis, P., Nikolic, M., Bleckwehl, T., Kolovos, P., et al. (2019). Modeling the Pathological LongRange Regulatory Effects of Human Structural Variation with Patient-Specific hiPSCs. Cell Stem Cell 24, 736-752.e12.

Law, C., Cheung, P., and Adhvaryu, K. (2015). Chemical "Diversity" of Chromatin Through Histone Variants and Histone Modifications. Curr. Mol. Biol. Reports 1, 39-59.

Lawrence, M., Daujat, S., and Schneider, R. (2016). Lateral Thinking: How Histone Modifications Regulate Gene Expression. Trends Genet. 32, 42-56.

Lecot, P., Alimirah, F., Desprez, P.Y., Campisi, J., and Wiley, C. (2016). Context-dependent effects of cellular senescence in cancer development. Br. J. Cancer 114, 1180-1184.

Lee, S., and Schmitt, C.A. (2019). The dynamic nature of senescence in cancer. Nat. Cell Biol. 21, 94-101.

Lee, S., Nam, Y., Koo, J.Y. oun., Lim, D., Park, J., Ock, J., Kim, J., Suk, K., and Park, S.B. u. (2014). A small molecule binding HMGB1 and HMGB2 inhibits microglia-mediated neuroinflammation. Nat. Chem. Biol. 10, 1055-1060.

Lettice, L.A., Heaney, S.J.H., Purdie, L.A., Li, L., de Beer, P., Oostra, B.A., Goode, D., Elgar, G., Hill, R.E., and de Graaff, E. (2003). A long-range Shh enhancer regulates expression in the developing limb and fin and is associated with preaxial polydactyly. Hum. Mol. Genet. 12, 1725-1735.

Lettice, L.A., Williamson, I., Devenney, P.S., Kilanowski, F., Dorin, J., and Hill, R.E. (2014). Development of five digits is controlled by a bipartite long-range cis-regulator. Dev. 141, 1715-1725.

Li, B., Carey, M., and Workman, J.L. (2007). The Role of Chromatin during Transcription. Cell 128, 707-719.

Lieberman-aiden, E., Berkum, N.L. Van, Williams, L., Imakaev, M., Ragoczy, T., Telling, A., Amit, I., Lajoie, B.R., Sabo, P.J., Dorschner, M.O., et al. (2009). of the Human Genome. 33292, 289-294.

López-Otín, C., Blasco, M.A., Partridge, L., Serrano, M., and Kroemer, G. (2013). The hallmarks of aging. Cell 153, 1194.

Lupiáñez, D.G., Kraft, K., Heinrich, V., Krawitz, P., Brancati, F., Klopocki, E., Horn, D., Kayserili, H., Opitz, J.M., Laxova, R., et al. (2015). Disruptions of topological chromatin domains cause pathogenic rewiring of gene-enhancer interactions. Cell 161, 1012-1025. 
Maurano, M.T., Humbert, R., Rynes, E., Thurman, R.E., Haugen, E., Wang, H., Reynolds, A.P., Sandstrom, R., Qu, H., Brody, J., et al. (2012). Systematic localization of common disease-associated variation in regulatory DNA. Science (80-. ). 337, 1190-1195.

McConnell, B.B., Starborg, M., Brookes, S., and Peters, G. (1998). Inhibitors of cyclindependent kinases induce features of replicative senescence in early passage human diploid fibroblasts. Curr. Biol. 8, 351-354.

Melnik, S., Caudron-Herger, M., Brant, L., Carr, I.M., Rippe, K., Cook, P.R., and Papantonis, A. (2016). Isolation of the protein and RNA content of active sites of transcription from mammalian cells. Nat. Protoc. 11, 553-565.

Meyer, K., Hodwin, B., Ramanujam, D., Engelhardt, S., and Sarikas, A. (2016). Essential Role for Premature Senescence of Myofibroblasts in Myocardial Fibrosis. J. Am. Coll. Cardiol. 67, 2018-2028.

Mikkelsen, T.S., Ku, M., Jaffe, D.B., Issac, B., Lieberman, E., Giannoukos, G., Alvarez, P., Brockman, W., Kim, T.K., Koche, R.P., et al. (2007). Genome-wide maps of chromatin state in pluripotent and lineage-committed cells. Nature 448, 553-560.

Monte, E., Rosa-Garrido, M., Karbassi, E., Chen, H., Lopez, R., Rau, C.D., Wang, J., Nelson, S.F., Wu, Y., Stefani, E., et al. (2016). Reciprocal regulation of the cardiac epigenome by chromatin structural proteins Hmgb and Ctcf: Implications for transcriptional regulation. J. Biol. Chem. 291, 15428-15446.

Moore, J.M., Rabaia, N.A., Smith, L.E., Fagerlie, S., Gurley, K., Loukinov, D., Disteche, C.M., Collins, S.J., Kemp, C.J., Lobanenkov, V. V., et al. (2012). Loss of maternal CTCF is associated with peri-implantation lethality of CtCf null embryos. PLoS One 7.

Muñoz-Espín, D., Cañamero, M., Maraver, A., Gómez-López, G., Contreras, J., MurilloCuesta, S., Rodríguez-Baeza, A., Varela-Nieto, I., Ruberte, J., Collado, M., et al. (2013). XProgrammed cell senescence during mammalian embryonic development. Cell 155, 1104.

Nakamura, S., and Yoshimori, T. (2017). New insights into autophagosome-lysosome fusion. J. Cell Sci. 130, 1209-1216.

Narendra, V., Rocha, P.P., An, D., Raviram, R., Skok, J.A., Mazzoni, E.O., and Reinberg, D. (2015). CTCF establishes discrete functional chromatin domains at the Hox clusters during differentiation. Science (80-. ). 347, 1017-1021.

Narita, M. (2007). Cellular senescence and chromatin organisation. Br. J. Cancer 96, 686691. 
Narita, M., Nũnez, S., Heard, E., Narita, M., Lin, A.W., Hearn, S.A., Spector, D.L., Hannon, G.J., and Lowe, S.W. (2003). Rb-mediated heterochromatin formation and silencing of E2F target genes during cellular senescence. Cell 113, 703-716.

Narita, M., Narita, M., Krizhanovsky, V., Nuñez, S., Chicas, A., Hearn, S.A., Myers, M.P., and Lowe, S.W. (2006). A Novel Role for High-Mobility Group A Proteins in Cellular Senescence and Heterochromatin Formation. Cell 126, 503-514.

Naumova, N., Imakaev, M., Fudenberg, G., Zhan, Y., Lajoie, B.R., Mirny, L.A., and Dekker, J. (2013). Organization of the mitotic chromosome. Science (80-. ). 342, 948-953.

Nemeth, M.J., Curtis, D.J., Kirby, M.R., Garrett-Beal, L.J., Seidel, N.E., Cline, A.P., and Bodine, D.M. (2003). Hmgb3: An HMG-box family member expressed in primitive hematopoietic cells that inhibits myeloid and B-cell differentiation. Blood 102, 1298-1306.

Nemeth, M.J., Cline, A.P., Anderson, S.M., Garrett-Beal, L.J., and Bodine, D.M. (2005). $\mathrm{Hmgb3}$ deficiency deregulates proliferation and differentiation of common lymphoid and myeloid progenitors. Blood 105, 627-634.

Nishimura, K., Fukagawa, T., Takisawa, H., Kakimoto, T., and Kanemaki, M. (2009). An auxin-based degron system for the rapid depletion of proteins in nonplant cells. Nat. Methods $6,917-922$.

Nora, E.P., Goloborodko, A., Valton, A.L., Gibcus, J.H., Uebersohn, A., Abdennur, N., Dekker, J., Mirny, L.A., and Bruneau, B.G. (2017). Targeted Degradation of CTCF Decouples Local Insulation of Chromosome Domains from Genomic Compartmentalization. Cell 169, 930-944.e22.

O’Sullivan, R.J., Kubicek, S., Schreiber, S.L., and Karlseder, J. (2010). Reduced histone biosynthesis and chromatin changes arising from a damage signal at telomeres. Nat. Struct. Mol. Biol. 17, 1218-1225.

Ong, C.T., and Corces, V.G. (2014). CTCF: An architectural protein bridging genome topology and function. Nat. Rev. Genet. 15, 234-246.

Pallier C, Scaffidi, Chopineau-Proust S, Agresti A, Nordmann P, Bianchi M, M. V (2003). Association of Chromatin Proteins High Mobility Group Box (HMGB) 1 and HMGB2 with Mitotic Chromosomes. 14, 3470-3481.

Phillips-Cremins, J.E., Sauria, M.E.G., Sanyal, A., Gerasimova, T.I., Lajoie, B.R., Bell, J.S.K., Ong, C.T., Hookway, T.A., Guo, C., Sun, Y., et al. (2013). Architectural protein subclasses shape 3D organization of genomes during lineage commitment. Cell 153, 1281- 
References

1295.

Pickersgill, H., Kalverda, B., De Wit, E., Talhout, W., Fornerod, M., and Van Steensel, B. (2006). Characterization of the Drosophila melanogaster genome at the nuclear lamina. Nat. Genet. 38, 1005-1014.

Rada-Iglesias, A., Grosveld, F.G., and Papantonis, A. (2018). Forces driving the threedimensional folding of eukaryotic genomes. Mol. Syst. Biol. 14, 1-12.

Rao, S.S.P., Huntley, M.H., Durand, N.C., Stamenova, E.K., Bochkov, I.D., Robinson, J.T., Sanborn, A.L., Machol, I., Omer, A.D., Lander, E.S., et al. (2014). A 3D map of the human genome at kilobase resolution reveals principles of chromatin looping. Cell 159, 1665-1680.

Rao, S.S.P., Huang, S.C., Glenn St Hilaire, B., Engreitz, J.M., Perez, E.M., Kieffer-Kwon, K.R., Sanborn, A.L., Johnstone, S.E., Bascom, G.D., Bochkov, I.D., et al. (2017). Cohesin Loss Eliminates All Loop Domains. Cell 171, 305-320.e24.

Reeves, R. (2010). Nuclear functions of the HMG proteins. Biochim. Biophys. Acta - Gene Regul. Mech. 1799, 3-14.

Ronfani, L., Ferraguti, M., Croci, L., Ovitt, C.E., Schöler, H.R., Consalez, G.G., and Bianchi, M.E. (2001). Reduced fertility and spermatogenesis defects in mice lacking chromosomal protein Hmgb2. Development 128, 1265-1273.

Rosenbloom, K.R., Sloan, C.A., Malladi, V.S., Dreszer, T.R., Learned, K., Kirkup, V.M., Wong, M.C., Maddren, M., Fang, R., Heitner, S.G., et al. (2013). ENCODE Data in the UCSC Genome Browser: Year 5 update. Nucleic Acids Res. 41, 56-63.

Rowley, M.J., and Corces, V.G. (2018). Organizational principles of 3D genome architecture. Nat. Rev. Genet. 19, 789-800.

Ruf, S., Symmons, O., Uslu, V.V., Dolle, D., Hot, C., Ettwiller, L., and Spitz, F. (2011). Large-scale analysis of the regulatory architecture of the mouse genome with a transposonassociated sensor. Nat. Genet. 43, 379-387.

Rufini, A., Tucci, P., Celardo, I., and Melino, G. (2013). Senescence and aging: The critical roles of p53. Oncogene 32, 5129-5143.

Sage, J., Miller, A.L., Pérez-Mancera, P.A., Wysocki, J.M., and Jacks, T. (2003). Acute mutation of retinoblastoma gene function is sufficient for cell cycle re-entry. Nature 424, 223-228.

Saksouk, N., Simboeck, E., and Déjardin, J. (2015). Constitutive heterochromatin formation and transcription in mammals. Epigenetics and Chromatin 8, 1-17. 
Salama, R., Sadaie, M., Hoare, M., and Narita, M. (2014). Cellular senescence and its effector programs. Genes Dev. 28, 99-114.

De Sandre-Giovannoli, A., Bernard, R., Cau, P., Navarro, C., Amiel, J., Boccaccio, I., Lyonnet, S., Stewart, C.L., Munnich, A., Le Merrer, M., et al. (2003). Lamin A truncation in Hutchinson-Gilford progeria. Science (80-. ). 300, 2055.

Sang, L., Coller, H.A., and Roberts, J.M. (2008). Control of the reversibility of cellular quiescence by the transcriptional repressor HES1. Science (80-. ). 321, 1095-1100.

Schaub, M.A., Boyle, A.P., Kundaje, A., Batzoglou, S., and Snyder, M. (2012). Linking disease associations with regulatory information in the human genome. Genome Res. 22, 1748-1759.

Schoenfelder, S., and Fraser, P. (2019). Long-range enhancer-promoter contacts in gene expression control. Nat. Rev. Genet.

Schwarzer, W., Abdennur, N., Goloborodko, A., Pekowska, A., Fudenberg, G., Loe-Mie, Y., Fonseca, N.A., Huber, W., Haering, C.H., Mirny, L., et al. (2017). Two independent modes of chromatin organization revealed by cohesin removal. Nature 551, 51-56.

Shimi, T., Butin-Israeli, V., Adam, S.A., Hamanaka, R.B., Goldman, A.E., Lucas, C.A., Shumaker, D.K., Kosak, S.T., Chandel, N.S., and Goldman, R.D. (2011). The role of nuclear lamin B1 in cell proliferation and senescence. Genes Dev. 25, 2579-2593.

Shumaker, D.K., Dechat, T., Kohlmaier, A., Adam, S.A., Bozovsky, M.R., Erdos, M.R., Eriksson, M., Goldman, A.E., Khuon, S., Collins, F.S., et al. (2006). Mutant nuclear lamin A leads to progressive alterations of epigenetic control in premature aging. Proc. Natl. Acad. Sci. U. S. A. $103,8703-8708$.

Singh, V.P., and Gerton, J.L. (2015). Cohesin and human disease: Lessons from mouse models. Curr. Opin. Cell Biol. 37, 9-17.

Sofiadis, S., K., K., Nikolic, N., M., M., Zirkel, Z., A., A., Kargapolova, K., Y., Y., Josipovic, J., N., N., et al. (2019). HMGB1 as a rheostat of chromatin topology and RNA homeostasis on the path to senescence. BioRxiv Genomics 1-33.

van Steensel, B., and Belmont, A.S. (2017). Lamina-Associated Domains: Links with Chromosome Architecture, Heterochromatin, and Gene Repression. Cell 169, 780-791.

Storer, M., Mas, A., Robert-Moreno, A., Pecoraro, M., Ortells, M.C., Di Giacomo, V., Yosef, R., Pilpel, N., Krizhanovsky, V., Sharpe, J., et al. (2013). XSenescence is a developmental mechanism that contributes to embryonic growth and patterning. Cell 155, 1119. 


\section{References}

Štros, M. (2010). HMGB proteins: Interactions with DNA and chromatin. Biochim. Biophys. Acta - Gene Regul. Mech. 1799, 101-113.

Štros, M., Polanská, E., Štruncovă, S., and Pospíšilová, Š. (2009). HMGB1 and HMGB2 proteins up-regulate cellular expression of human topoisomerase II $\alpha$. Nucleic Acids Res. 37, 2070-2086.

Sun, L., Yu, R., and Dang, W. (2018). Chromatin architectural changes during cellular senescence and aging. Genes (Basel). 9.

Symmons, O., Uslu, V.V., Tsujimura, T., Ruf, S., Nassari, S., Schwarzer, W., Ettwiller, L., and Spitz, F. (2014). Functional and topological characteristics of mammalian regulatory domains. Genome Res. 24, 390-400.

Symmons, O., Pan, L., Remeseiro, S., Aktas, T., Klein, F., Huber, W., and Spitz, F. (2016). The Shh Topological Domain Facilitates the Action of Remote Enhancers by Reducing the Effects of Genomic Distances. Dev. Cell 39, 529-543.

Szabo, Q., Bantignies, F., and Cavalli, G. (2019). Principles of genome folding into topologically associating domains. Sci. Adv. 5.

Teves, S.S., An, L., Hansen, A.S., Xie, L., Darzacq, X., and Tjian, R. (2016). A dynamic mode of mitotic bookmarking by transcription factors. Elife 5, 1-24.

Tominaga-Yamanaka, K., Abdelmohsen, K., Martindale, J.L., Yang, X., Taub, D.D., and Gorospe, M. (2012). Nf90 coordinately represses the senescence-associated secretory phenotype. Aging (Albany. NY). 4, 695-708.

Tse, C., Shoemaker, A.R., Adickes, J., Anderson, M.G., Chen, J., Jin, S., Johnson, E.F., Marsh, K.C., Mitten, M.J., Nimmer, P., et al. (2008). ABT-263: A potent and orally bioavailable Bcl-2 family inhibitor. Cancer Res. 68, 3421-3428.

Ueda, T., and Yoshida, M. (2010). HMGB proteins and transcriptional regulation. Biochim. Biophys. Acta - Gene Regul. Mech. 1799, 114-118.

Vietri Rudan, M., Barrington, C., Henderson, S., Ernst, C., Odom, D.T., Tanay, A., and Hadjur, S. (2015). Comparative Hi-C Reveals that CTCF Underlies Evolution of Chromosomal Domain Architecture. Cell Rep. 10, 1297-1309.

Wang, J., Jia, S.T., and Jia, S. (2016). New Insights into the Regulation of Heterochromatin. Trends Genet. 32, 284-294.

Watanabe, S., Kawamoto, S., Ohtani, N., and Hara, E. (2017). Impact of senescenceassociated secretory phenotype and its potential as a therapeutic target for senescence- 
associated diseases. Cancer Sci. 108, 563-569.

Williamson, I., Lettic, L.A., Hill, R.E., and Bickmore, W.A. (2016). Shh and ZRS enhancer colocalisation is specific to the zone of polarising activity. Dev. 143, 2994-3001.

Williamson, I., Kane, L., Devenney, P.S., Anderson, E., Kilanowski, F., Hill, R.E., Bickmore, W.A., and Lettice, L.A. (2019). Developmentally regulated Shh expression is robust to TAD perturbations. BioRxiv 609941.

de Wit, E., and de Laat, W. (2012). A decade of 3C technologies: Insights into nuclear organization. Genes Dev. 26, 11-24.

de Wit, E., Vos, E.S.M., Holwerda, S.J.B., Valdes-Quezada, C., Verstegen, M.J.A.M., Teunissen, H., Splinter, E., Wijchers, P.J., Krijger, P.H.L., and de Laat, W. (2015). CTCF Binding Polarity Determines Chromatin Looping. Mol. Cell 60, 676-684.

Wutz, G., Várnai, C., Nagasaka, K., Cisneros, D.A., Stocsits, R.R., Tang, W., Schoenfelder, S., Jessberger, G., Muhar, M., Hossain, M.J., et al. (2017). Topologically associating domains and chromatin loops depend on cohesin and are regulated by CTCF, WAPL, and PDS5 proteins. EMBO J. 36, 3573-3599.

Xu, M., Pirtskhalava, T., Farr, J.N., Weigand, B.M., Palmer, A.K., Weivoda, M.M., Inman, C.L., Ogrodnik, M.B., Hachfeld, C.M., Fraser, D.G., et al. (2018). Senolytics improve physical function and increase lifespan in old age. Nat. Med. 24, 1246-1256.

Xue, W., Zender, L., Miething, C., Dickins, R.A., Hernando, E., Krizhanovsky, V., CordonCardo, C., and Lowe, S.W. (2007). Senescence and tumour clearance is triggered by p53 restoration in murine liver carcinomas. Nature 445, 656-660.

Yosef, R., Pilpel, N., Tokarsky-Amiel, R., Biran, A., Ovadya, Y., Cohen, S., Vadai, E., Dassa, L., Shahar, E., Condiotti, R., et al. (2016). Directed elimination of senescent cells by inhibition of BCL-W and BCL-XL. Nat. Commun. 7, 1-11.

Zhang, R., Poustovoitov, M. V., Ye, X., Santos, H.A., Chen, W., Daganzo, S.M., Erzberger, J.P., Serebriiskii, I.G., Canutescu, A.A., Dunbrack, R.L., et al. (2005). Formation of macroH2A-containing senescence-associated heterochromatin foci and senescence driven by ASF1a and HIRA. Dev. Cell 8, 19-30.

Zhang, W., Li, J., Suzuki, K., Qu, J., Wang, P., Zhou, J., Liu, X., Ren, R., Xu, X., Ocampo, A., et al. (2015). A Werner syndrome stem cell model unveils heterochromatin alterations as a driver of human aging. Science (80-. ). 348, 1160-1163.

Zirkel, A., Nikolic, M., Sofiadis, K., Mallm, J.P., Brackley, C.A., Gothe, H., Drechsel, O., 
References

Becker, C., Altmüller, J., Josipovic, N., et al. (2018). HMGB2 Loss upon Senescence Entry Disrupts Genomic Organization and Induces CTCF Clustering across Cell Types. Mol. Cell 70, 730-744.e6. 


\section{Curriculum Vitae}

\section{Konstantinos C. Sofiadis (MSc)}

\section{Personal information}

Nationality: Greek

Birth Date: 3 April 1990

Email:

sofiadisk@gmail.com
Work Address: Robert Koch Strasse 40, Department of Pathology, University Medical Center Göttingen Germany

Permanent Address: Gosslestrsse 77, Goettingen

Home Address: Pantazopoulou 25, Sidirokastro, Serres, Greece

\section{Education}

October 2015 Present

October 2013-September 2015

September 2008-September 2013

\section{PhD candidate in System Biology of Chromatin}

Department of Pathology, University Medical Center Göttingen Germany

PhD thesis: Dissecting the molecular mechanism and spatiotemporal dynamics controlling senescence entry

MSc in Molecular Biology and

Biomedicine Faculty of Medicine, University of Crete

Joint degree with Dept of Biology, Univ. of Crete

Thesis: Regulation of miR-155 in response to leptin macrophages

Supervisor: Dr. Christos Tsatsanis, Faculty of Medicine,

University of Crete

\section{BSc in Biology}

University of Crete, Dept of Biology, Heraklion, Greece

Thesis: Role of DCLs in PTGS transgenic GFP transgene in

Nicotiana benthamiana.

Supervisor: Kriton Kalantidis 


\section{Research Experience}

September 2008-September 2013 Master Thesis

Clinical Chemistry laboratroy Supervisor: Dr Christos Tsatsanis

Faculty of Medicine, University of Crete, Heraklion, Greece,

Epigenetic changes and regulation of miR-155 in response to leptin in

macrophages

August 2014-September 2014

\section{Two-month technical skills training}

Clinical Chemistry lab. Supervisor: Dr Christos Tsatsanis

Faculty of Medicine, University of Crete, Heraklion, Greece

Subject: Regulation of the metabolic inflammation through the differentiation and activation of the adipocytes.

June 2014-July 2014 Two- month technical skills training

Gene Regulation in the Immune System Lab. Supervisor: Dr Charalambos

Spilianakis

Institute of Molecular Biology and Biotechnology (IMBB), Foundation for Research and Technology-Hellas (FORTH).

Subject: Sub-nuclear localization of inflammatory miRNAs in immunological cell types.

March 2014-May 2014 Two -month technical skills training

Division of Infections and Immunity Lab. Supervisor: Dr Athanasios Loukeris Institute of Molecular Biology and Biotechnology (IMBB), Foundation for Research and Technology-Hellas (FORTH).

Subject: The role of serpins in the blockage of parasite's transmission.

September 2012-September

\section{Diploma Thesis}

Molecular Biology of Plant Lab. Supervisor: Dr Kriton Kalantidis

Department of Biology, University of Crete \& Institute of Molecular Biology and Biotechnology(IMBB), Foundation for Research and Technology- Hellas

(FORTH) Heraklion, Greece

Subject: Role of DCLs in PTGS transgenic GFP transgene in Nicotiana benthamiana.Supervisor: Dr. Christos Tsatsanis, Faculty of Medicine, University of Crete 


\section{Publications}

HMGB2 Loss upon Senescence Entry Disrupts Genomic Organization and Induces CTCF Clustering across Cell Types.

Zirkel A*, Nikolic M*, Sofiadis K*, Mallm JP, Brackley CA, Gothe H, Drechsel O, Becker C, Altmüller J, Josipovic N, Georgomanolis T,Brant L, Franzen J, Koker M, Gusmao EG, Costa IG, Ullrich RT, Wagner W, Roukos V, Nürnberg P, Marenduzzo D, Rippe K, Papantonis A. Mol Cell. 2018 May 17;70(4):730-744296

*Authors equally contributed

HMGB1 as a rheostat of chromatin topology and RNA homeostasis on the path to senescence.

Konstantinos Sofiadis, Milos Nikolic, Anne Zirkel, Yulia Kargapolova, Natasa Josipovic, Antonis Papadakis, Eduardo G. Gusmao, Athanasia Mizi, Theodore Georgomanolis, Mirjam Koker, Roland T. Ullrich, Janine Altmüller, Peter Nürnberg, Andreas Beyer \& Argyris Papantonis. Published in bioarxiv

Cutting a Long Intron Short: Recursive Splicing and Its Implications.

Georgomanolis T, Sofiadis K, Papantonis A. Front Physiol. 2016 Nov 29;7:598. eCollection 2016.

12 - Transcription Factories as Spatial and Functional Organization Nodes Nuclear Architecture and Dynamics Konstantinos Sofiadis, Argyris Papantonis. 2018, Pages 283-286 
